Teoremas ergódicos

dos pontos de vista probabilístico e topológico

Lucas Amorim Vilas Boas

\author{
DISSERTAÇÃO APRESENTADA \\ $\mathrm{AO}$ \\ Instituto de Matemática e Estatística \\ DA \\ UniversidAdE DE SÃO PAUlo \\ PARA \\ OBTENÇÃO DO TÍTULO \\ DE \\ MESTRE EM CiÊNCIAS \\ Programa: Matemática Aplicada \\ Orientador: Prof. Dr. Fabio Armando Tal
}

Durante o desenvolvimento deste trabalho o autor recebeu auxílio financeiro da CNPq

São Paulo, Junho de 2018 



\title{
Ergodic theorems from probabilistic and topological viewpoints
}

\author{
Lucas Amorim Vilas Boas
}

\author{
DISSERTATION PRESENTED \\ TO THE \\ Institute of Mathematics And Statistics \\ OF THE \\ UNIVERSITY OF SÃO PAULO \\ TO \\ FULFILL THE REQUIREMENTS OF THE DEGREE \\ OF \\ MASTER IN SCIENCE
}

Program: Applied Mathematics

Advisor: Prof. Dr. Fabio Armando Tal

During the elaboration of this work the author had the financial support of CNPq

São Paulo, June 2018 



\title{
Teoremas ergódicos \\ dos pontos de vista probabilístico e topológico
}

\begin{abstract}
Esta versão da dissertação contém as correções e alterações sugeridas pela Comissão Julgadora durante a defesa da versão original do trabalho, realizada em 17/05/2018. Uma cópia da versão original está disponível no Instituto de Matemática e Estatística da Universidade de São Paulo.
\end{abstract}

Comissão Julgadora:

- Prof. Dr. Fabio Armando Tal (orientador) - IME-USP

- Prof. Dr. Alejandro Kocsard - IME-UFF

- Prof. Dr. Ricardo dos Santos Freire Junior - IME-USP 



\section{Ergodic theorems from probabilistic and topological viewpoints}

This version of the dissertation incorporates improvements suggested by the Examining Board in the event of the defense of the original version of this work, in $05 / 17 / 2018$. A copy of the original version is available at the Institute of Mathematics and Statistics of the University of São Paulo.

Examining Board:

- Prof. Fabio Armando Tal, PhD (advisor) - IME-USP

- Prof. Alejandro Kocsard, PhD - IME-UFF

- Prof. Ricardo dos Santos Freire Junior, PhD - IME-USP 



\section{Acknowledgments}

First of all, I would like to thank my advisor Prof. Fabio Armando Tal, for this opportunity and for his kindness, guidance and shared knowledge. I would also like to thank Prof. Jorge Sotomayor and Prof. Salvador Zanata, who also introduced me to the quest of dynamical systems. In fact, the structural role of the Brazilian dynamical systems community is to be acknowledged.

Nonetheless, I would like to thank the support of my family and friends and the lasting care and patience of my girlfriend Brenda Poubel during this journey. 


\section{Resumo}

\section{AMORIM, L. V. B. Teoremas ergódicos dos pontos de vista probabilístico e topológico.}

2018. 87 f. Dissertação (Mestrado) - Instituto de Matemática e Estatística, Universidade de São Paulo, São Paulo, 2018.

Teoremas ergódicos são resultados medida-teóricos clássicos em sistemas dinâmicos ou, mais precisamente, teoria ergódica. Eles afirmam que a convergência das médias de Birkhoff é típica, em um sentido de medida. Este trabalho objetiva explicar como esses resultados podem ser reinterpretados à luz da topologia e da teoria das probabilidades.

A primeira relação é apresentada por meio de um análogo em categoria de Baire de uma versão habitual do teorema ergódico de Birkhoff (assumindo ergodicidade). Ao invés de convergência das médias de Birkhoff, o comportamento topologicamente típico será oposto: as médias não convergirão de modo dramático.

A segunda relação é apresentada examinando como a lei dos grandes números interage com o teorema ergódico de Birkhoff (assumindo ergodicidade). A lei dos grandes números pode ser obtida como corolário do teorema ergódico de Birkhoff. Entretanto, ela permite um novo ponto de vista, pois mantém as conclusões do teorema ergódico de Birkhoff (assumindo ergodicidade) mesmo no caso não-ergódico, ao custo de que haja um certo tipo de independência.

Palavras-chave: teorema ergódico, análogo topológico, categoria de Baire, lei dos grandes números. 


\section{Abstract}

AMORIM, L. V. B. Ergodic theorems from probabilistic and topological viewpoints. 2018. 87 p. Dissertation (Master) - Instituto de Matemática e Estatística, Universidade de São Paulo, São Paulo, 2018.

Ergodic theorems are classic measure theoretical results in dynamical systems or, more precisely, ergodic theory. They state that the convergence of Birkhoff averages is typical, in a measuretheoretical sense. This work aims to explain how these results can be re-interpreted in light of topology and probability theory.

The first relationship is presented through a Baire category analogue of a standard version of Birkhoff's ergodic (assuming ergodicity). Instead of convergence of Birkhoff averages, the topological typical behavior will be the opposite: averages do not converge in a dramatic way.

The second relationship is presented by examining how the law of large numbers interacts with Birkhoff's ergodic theorem (assuming ergodicity). The law of large numbers can be obtained as corollary of Birkhoff's ergodic theorem. However, the law provides a new point of view, as it guarantees the conclusions of Birkhoff's ergodic theorem (assuming ergodicity) will hold even in the non-ergodic case, at the cost of requiring some sort of independence.

Keywords: ergodic theorem, topological analogue, Baire category, law of large numbers. 


\section{Contents}

$\begin{array}{ll}\text { List of abbreviations } & \text { ix }\end{array}$

List of symbols $\quad$ xi

1 Introduction $\quad 1$

2 Prerequisites $\quad 3$

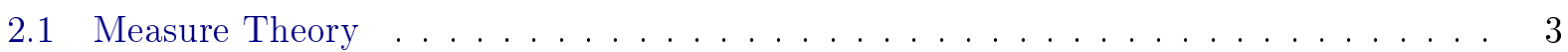

2.2 Functional analysis and the space of measures $\ldots \ldots \ldots \ldots \ldots$

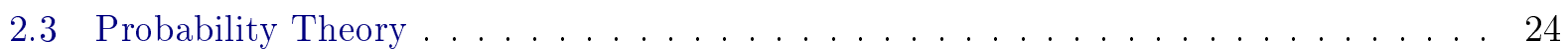

2.4 Dynamical Systems and Ergodic Theory . . . . . . . . . . . . . . . . . . 33

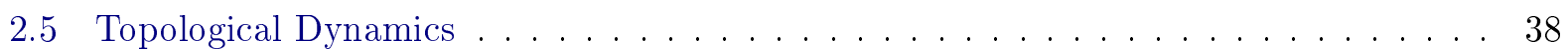

3 Birkhoff's Ergodic Theorems:

Measure Theoretical and Topological Analogues $\quad 45$

3.1 Birkhoff Ergodic Theorem (Measure Theoretical) . . . . . . . . . . . . . . . . . . . 45

3.2 Birkhoff Ergodic Theorem Topological Analogue . . . . . . . . . . . . . . . . . 61

4 Law of Large Numbers:

From Probability to Ergodic Theory $\quad 69$

4.1 Unifying Notation and Framework . . . . . . . . . . . . . . . . . . 69

4.2 Law of Large Numbers . . . . . . . . . . . . . . . . . . . . 74

5 Final Remarks $\quad 81$

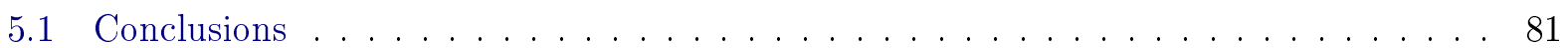

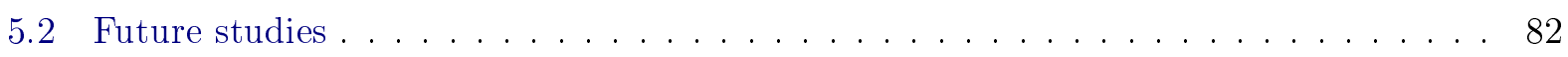

$\begin{array}{ll}\text { Bibliography } & 83\end{array}$ 
viii CONTENTS 


\title{
List of abbreviations
}

\author{
LCH Locally compact Hausdorff space \\ SM Separable metric space \\ LCH + SM Locally compact Hausdorff separable metric space \\ BET Birkhoff's ergodic theorem \\ IID Independent and identically distributed \\ LLN Law of large numbers \\ a.e. Almost everywhere \\ a.a. Almost all
}




\section{List of symbols}

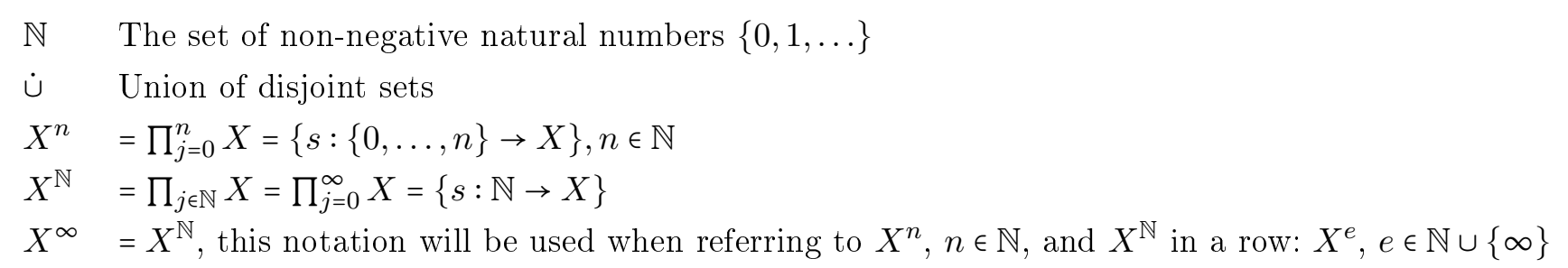




\section{Chapter 1}

\section{Introduction}

Interactions between measurable and topological dynamics, as well as between probability theory and ergodic theory, are known to be fruitful.

Many theorems in ergodic theory can be enriched by tracing analogues to topological dynamics. A paradigmatic case is an analogy between Poincare's recurrence and Birkhoff's topological recurrence.

On the other hand, many definitions and results in ergodic theory are very naturally understood in probabilistic terms, due to the very nature of objects making up ergodic theory.

The idea is to strengthen these connections, making them work for ergodic theorems. For our concerns, Birkhoff's ergodic theorem is the major one. Later, this theorem will be presented indepth. For now, it can be summarized as stating measure-theoretical typical convergence of the so-called Birkhoff averages.

One objective of this dissertation is to present a topological (or a topological dynamics) analogue to Birkhoff's ergodic theorem. The major issue is that measure-theoretical and topological structures do not always translate perfectly.

The kind of topological dynamics in which we are looking for analogues is the abstract one, whose topological structure and dynamical system acting on it are quite general. Distributional properties of orbits in those systems, to be understood roughly as properties of the set of measures accumulated by averages of Diracs supported along orbits, will play a key role. These properties are to be understood as topological in the sense that they do not require invariant measures. Also, we will often try to verify whether those and other properties hold typically in the sense of Baire category. Each of these subjects will be addressed during this text. See sections 2.5 and 3.2.

Another objective of this dissertation is to present a probability theory analogue to Birkhoff's ergodic theorem. In this case, the analogue is well-known, namely, the law of large numbers. Our aim is to scrutinize how these theorems relate. The major issue is that, in probability theory, no system exists at all. It is necessary to create a conceptual bridge between ergodic and probability theories. Our inclination will be that of a dynamicist, i.e., using the bridge to adapt content from probability theory to ergodic theory. In particular, the law of large number will be adapted and this adaptation compared to Birkhoff's ergodic theorem.

To create this bridge, a lot is discussed in terms of approaching a dynamical system as a stochastic process and, conversely, approaching a stochastic process as modeled by a dynamical system. Another central feature is to interpret independence (and identical distribution) in the context of dynamics. Again, each of these subjects will be addressed during this text. See sections 2.3 and 4.1 .

To give a glimpse of what is coming, the topological analogue of Birkhoff's ergodic theorem (assuming ergodicity), to be presented later in the text, shows that the typical behavior of Birkhoff averages is, perhaps surprisingly, a dramatic non-convergence. This discussion is carried in section 3.2 and is due to [Win10].

Also, we show that the law of large numbers follows from Birkhoff's ergodic theorem. However, the law provides a new point of view, as it guarantees the conclusions of Birkhoff's ergodic theorem 
(assuming ergodicity) will hold even in the non-ergodic case, at the cost of requiring some sort of independence. This discussion is carried in section 4.2 and its proof relies heavily on the previous conceptual bridge, both of which are presented by the author in an original way. Even though no reference is used, these subjects some how standard implicit knowledge for the mathematician working on this interface. 


\section{Chapter 2}

\section{Prerequisites}

In this chapter, during sections 2.1 and 2.2 , it is assumed the reader is familiar with measure theory and functional analysis as in [Fol07]. During section 2.3, it is assumed the reader is familiar with probability theory as in the first three chapters of [Çn11]. During section 2.4, it is assumed the reader is familiar with dynamical systems and ergodic theory as in the first five chapters of [VO16]. Unless locally mentioned, these texts are references for each respective section. The last section, 2.5, about topological dynamical systems, is a collection of results from many sources, according to the taste of the author. Those proofs are quite standard, but some of them, about topological dynamics per se, were adapted.

The exposition in each section summarizes many aspects of the texts mentioned above but do not prove every proposition in detail, especially in sections 2.1, 2.2 and 2.3, where technical proofs are omitted and only important proofs are sketched or indicated. However, proofs start to show up complete in sections 2.4 and 2.5, as we move into the core subject of this dissertation.

The experienced reader can skip this chapter and consult it only when necessary. The reader lacking any prerequisite can fulfill it during this chapter, at least to the extent necessary to make sense of the rest of the dissertation. Sections 2.4 and 2.5, however, are pretty much complete and offer a decent basis for the results they are presenting.

\subsection{Measure Theory}

During this section, we review some measure theory in order to align notation and the statement of major results. Proofs of purely technical content are omitted, while proofs of instructive or important results are briefly sketched or indicated.

For our convenience, we define the extended reals $[-\infty, \infty]=\overline{\mathbb{R}}:=\{-\infty\} \cup \mathbb{R} \cup\{\infty\}$. The order in $[-\infty, \infty]$ is given by $-\infty<x<\infty, \forall x \in \mathbb{R}$. Therefore every set in $[-\infty, \infty]$ is bounded and has infimum and supremum, hence every sequence in $[-\infty, \infty]$ has $\lim \sup _{n}=\inf _{k \geq 1} \sup _{n \geq k}$ and $\liminf \operatorname{in}_{n}=\sup _{k \geq 1} \inf _{n \geq k}$. The topology in $[-\infty, \infty]$ is that generated by the subbasis consisting of the open intervals, $(a, b)=\{x \in[-\infty, \infty]: a<x<b\},(a, \infty]=\{x \in[-\infty, \infty]: x>a\},[-\infty, b)=\{x \in$ $[-\infty, \infty]: x<b\}$ and $[-\infty, \infty]$, with $a, b \in[-\infty, \infty]$. Closed and semi-closed intervals in $[-\infty, \infty]$ are defined naturally. A sequence in this topology converges if, and only if, liminf and limsup are coincident. The arithmetic operations $+:[-\infty, \infty] \times[-\infty, \infty] \backslash\{(-\infty, \infty),(\infty,-\infty)\} \rightarrow[-\infty, \infty]$ and $\cdot:[-\infty, \infty] \times[-\infty, \infty] \rightarrow[-\infty, \infty]$ are defined (extending the real operations) as: $x+ \pm \infty= \pm \infty=$ $\pm \infty+x, \forall x \in \mathbb{R} ; \pm \infty+ \pm \infty= \pm \infty ; x \cdot \pm \infty= \pm \infty= \pm \infty \cdot x, \forall x>0, x \cdot \pm \infty=\mp \infty= \pm \infty \cdot x, \forall x<0$ and $0 \cdot \pm \infty=0= \pm \infty \cdot 0$.

We then define $\sigma$-algebras, collections of subsets of a space $X$ (whose elements are called measurable sets) on which we can conveniently prescribe specific functions assigning values in $[0, \infty]$, to be interpreted as generalized areas or volumes.

Definition 2.1.1 (Algebra and $\sigma$-algebra). Let $X$ be a nonempty set and $\mathcal{A} \subset \mathcal{P}(X)$ (the set of parts of $X$ ). A collection $\mathcal{A}$ is an algebra (a $\sigma$-algebra) on $X$ if: (i) $\varnothing \in \mathcal{A}$, (ii) $A \in \mathcal{A} \Rightarrow A^{\mathrm{c}} \in \mathcal{A}$, (iii) if $A_{n} \in \mathcal{A}$, for $n \in\{0, \ldots, k\}$ (or $\left.n \geq 0\right)$, then $\cup_{n=0}^{k} A_{n} \in \mathcal{A}\left(\right.$ or $\left.\cup_{n=0}^{\infty} A_{n} \in \mathcal{A}\right)$. 
It is easy to note that $\mathcal{P}(X)$ and $\{\varnothing, X\}$ are $\sigma$-algebras and that the arbitrary intersection of $\sigma$-algebras on $X$ is a $\sigma$-algebra on $X$. It is then possible to define the $\sigma$-algebra generated by $\mathcal{Y} \subset \mathcal{P}(X)$, as the intersection of all $\sigma$-algebras containing $\mathcal{Y}, \sigma(\mathcal{Y}):=\bigcap_{\mathcal{Y} \subset \mathcal{A}} \mathcal{A}$. It is well defined (as $\mathcal{P}(X)$ always makes the intersection non-trivial), unique and understood as smallest $\sigma$-algebra containing $\mathcal{Y}$. The definition of the algebra generated by $\mathcal{Y} \subset \mathcal{P}(X)$ is analogous and denoted by $\mathcal{A}(\mathcal{Y})$.

If $(X, \tau)$ is a topological space, the $\sigma$-algebra $\sigma(\tau)$ is called the Borel $\sigma$-algebra of $X$, denoted $\mathcal{B}_{X}$. Its elements are called Borelians sets. When dealing with a topological space, we will implicitly assume it is equipped with a Borel $\sigma$-algebra, unless otherwise stated.

A pair $(X, \mathcal{A})$, where $X$ is a nonempty set and $\mathcal{A}$ is a $\sigma$-algebra on $X$, is called a measurable space.

Definition 2.1.2 (Measure). Let $(X, \mathcal{A})$ be a measurable space. A measure $\mu$ on $(X, \mathcal{A})$ (or $X$, or $\mathcal{A}$, if clear from the context) is a function $\mu: \mathcal{A} \rightarrow[0, \infty]$ such that: (i) $\mu(\varnothing)=0$ and (ii) if $\left(A_{n}\right)_{n \geq 0}$ is a sequence of disjoint sets in $\mathcal{A}$, then, denoting $\dot{\cup}$ a union of disjoint sets, $\mu\left(\dot{\cup}_{n \geq 0} A_{n}\right)=\sum_{n \geq 0} \mu\left(A_{n}\right)$.

A measurable space $(X, \mathcal{A})$ equipped with a measure $\mu$ on $(X, \mathcal{A}),(X, \mathcal{A}, \mu)$, is called a measure space. If $X$ is a topological space, a measure on $\left(X, \mathcal{B}_{X}\right)$ is called Borelian measure on $X$.

Let $(X, \mathcal{A}, \mu)$ be a measure space. We say $\mu$ is: (i) a probability if $\mu(X)=1$, (ii) finite if $\mu(X)<\infty$, (iii) $\sigma$-finite if $\exists\left(A_{n}\right)_{n \geq 0}$ a sequence in $\mathcal{A}$ such that $X=\cup_{n} A_{n}$ and $\mu\left(A_{n}\right)<\infty, \forall n \geq 0$ and (iv) semifinite if for each $A \in \mathcal{A}$ such that $\mu(A)=\infty, \exists E \in \mathcal{A}, E \subset A$, such that $0<\mu(E)<\infty$.

The following proposition summarizes the basic working properties of measure spaces.

Proposition 2.1.3 (Properties of measures). Let $(X, \mathcal{A}, \mu)$ be a measure space. Then:

(i) (Monotonicity) for any $A, B \in \mathcal{A}, A \subset B, \mu(A) \leq \mu(B)$

(ii) (Subadditivity) for any sequence $\left(A_{n}\right)_{n \geq 0}$ in $\mathcal{A}, \mu\left(\cup_{n} A_{n}\right) \leq \sum_{n} \mu\left(A_{n}\right)$

(iii) (Continuity from below) for any increasing sequence $\left(A_{n}\right)_{n \geq 0}$ in $\mathcal{A}$ (with $A_{n} \subset A_{n+1}$, for all $n \geq 0), \mu\left(\cup_{n} A_{n}\right)=\lim _{n} \mu\left(A_{n}\right)$

(iv) (Continuity from above) for any decreasing sequence $\left(A_{n}\right)_{n \geq 0}$ in $\mathcal{A}$, (with $A_{n} \supset A_{n+1}$, for all $n \geq 0)$ and such that $\mu\left(A_{j}\right)<\infty$ for some $j \geq 0, \mu\left(\cap_{n} A_{n}\right)=\lim _{n} \mu\left(A_{n}\right)$.

Dealing with a finite measure space, we can count on a very general approximation property, using elements from a generating algebra.

Proposition 2.1.4 (Approximating with a generating algebra). Let $(X, \mathcal{A}, \mu)$ be a finite measure space and $\mathcal{A}$ an algebra on $X$ generating $\mathcal{A}$. Then $\forall E \in \mathcal{A}, \epsilon>0, \exists D \in \mathcal{A}$ such that $\mu(E \triangle D)<\epsilon$, where $E \triangle D=(E \backslash D) \dot{\cup}(D \backslash E)$.

On a measure space $(X, \mathcal{A}, \mu)$, if a property $P$ holds for every $x \in X$ except possibly on a set $E$ with $\mu(E)=0$ (a null set), we say $P$ is true $\mu$-almost everywhere (abbreviated $\mu$-a.e.), or for $\mu$-almost every $x \in X$ (abbreviated $\mu$-a.e. $x \in X$ ).

A measure space $(X, \mathcal{A}, \mu)$ such that every subset of a null set is measurable (hence a null set, by monotonicity) is called complete.

Proposition 2.1.5 (Completing a measure space). Let $(X, \mathcal{A}, \mu)$ be a measure space, $\mathcal{N}=\{N \subset X$ : $\mu(N)=0\}$ and $\overline{\mathcal{A}}=\{B \subset X: \exists A \in \mathcal{A}, N \in \mathcal{N}, M \subset N$, such that $B=A \cup M\}$. Then $\overline{\mathcal{A}}$ is a $\sigma$-algebra (called the completion of $\mathcal{A}$ with respect to $\mu$ ) and there is a unique extension $\bar{\mu}$ of $\mu$ (called the completion of $\mu$ ), that makes $(X, \overline{\mathcal{A}}, \bar{\mu})$ a complete measure space, namely $\bar{\mu}(B)=\bar{\mu}(A \cup M)=\mu(A)$.

We now focus on the first tool used to construct measures, the so-called Caratheodory construction (the second is Riesz-Markov representation theorem). In special, we address the construction of Lesbegue-Stieltjes measures on $\mathbb{R}$.

Definition 2.1.6 (Outer measure). Let $X$ be a set. An outer measure $\mu^{*}$ on $X$ is a set function on $\mathcal{P}(X), \mu^{*}: \mathcal{P}(X) \rightarrow[0, \infty]$ such that (i) $\mu^{*}(\varnothing)=0$, (ii) $C \subset D \subset X \Rightarrow \mu^{*}(C) \leq \mu^{*}(D)$ and (iii) if $\left(C_{n}\right)_{n \geq 0}$ is a sequence of subsets of $X$, then $\mu^{*}\left(\cup_{n \geq 0} C_{n}\right) \leq \sum_{n \geq 0} \mu^{*}\left(C_{n}\right)$. 
Proposition 2.1.7 (Creating outer measures from pre-outer measures). Let $X$ be a set and $\mathcal{S} \subset$ $\mathcal{P}(X)$ be such that (i) $\varnothing \in \mathcal{S}$ and (ii) $\exists\left(S_{n}\right)_{n \geq 0}$ a sequence of elements in $\mathcal{S}$ such that $\cup_{n} S_{n}=X(\mathcal{S}$ is to be a family of "simple" sets that we know how to measure, e.g, a set of intervals/squares/cubes) and $\mu^{0}: \mathcal{S} \rightarrow[0, \infty]$ be such that $\mu^{0}(\varnothing)=0\left(\mu^{0}\right.$ is called a pre-outer measure and simply assign a prototypical measure).

For each $C \subset X$, define $\mu^{*}(C)=\inf \left\{\sum_{n} \mu^{0}\left(S_{n}\right):\left(S_{n}\right)_{n \geq 0}\right.$ is a sequence in $\mathcal{S}$ such that $\left.C \subset \cup_{n} S_{n}\right\}$. Then $\mu^{*}$ is an outer measure, called the outer measure induced by $\mu^{0}$.

We are going to induce a measure from an outer measure (or ultimately from a pre-outer measure).

First we define a set $A \subset X$ to be $\mu^{*}$-measurable if "splits well every other set" with respect to $\mu^{*}$, i.e., if $\mu^{*}(E)=\mu^{*}(E \cap A)+\mu^{*}\left(E \cap A^{\mathrm{c}}\right), \forall E \subset X$. Since the inequality $(\leq)$ is immediate, it is enough to check that $\mu^{*}(E) \geq \mu^{*}(E \cap A)+\mu^{*}\left(E \cap A^{\mathrm{c}}\right), \forall E \subset X$ such that $\mu^{*}(E)<\infty$ (since, when $\mu^{*}(E)=\infty$, no checking is required).

Theorem 2.1.8 (Caratheodory's theorem - creating measures from outer measures). Let $X$ be a set and $\mu^{*}$ an outer measure on $X$. Then

(i) $\left\{A \subset X: A\right.$ is $\mu^{*}$-measurable $\}$ is a $\sigma$-algebra on $X$, denoted $\sigma\left(\mu^{*}\right)$.

(ii) $\left.\mu^{*}\right|_{\sigma\left(\mu^{*}\right)}$ is a measure on $\left(X, \sigma\left(\mu^{*}\right)\right)$, called the measure induced by $\mu^{*}$

(iii) for each $C \subset X$ such that $\mu^{*}(C)=0$, it holds that $C \in \sigma\left(\mu^{*}\right)$; therefore $\left(X, \sigma\left(\mu^{*}\right),\left.\mu^{*}\right|_{\sigma\left(\mu^{*}\right)}\right)$ is complete. Denote $\left.\mu^{*}\right|_{\sigma\left(\mu^{*}\right)}$ by $\hat{\mu}$.

The preceding theorem will be applied to a specific case, which will be useful for constructing Lesbegue-Stieltjes measures on $\mathbb{R}$ : extending pre-measures (on an algebra) to measures (on the $\sigma$-algebra generated by that algebra).

Definition 2.1.9 (Pre-measures). Let $X$ a set and $\mathcal{A}$ be an algebra on $X$. A pre-measure $\mu_{0}$ on $(X, \mathcal{A})$ (or $X$, or $\mathcal{A}$, if clear from the context) is a function $\mu_{0}: \mathcal{A} \rightarrow[0, \infty]$ such that: (i) $\mu_{0}(\varnothing)=0$ and (ii) if $\left(A_{n}\right)_{n \geq 0}$ is a sequence of disjoint sets in $\mathcal{A}$ such that $\dot{\cup}_{n \geq 0} A_{n} \in \mathcal{A}$, then $\mu_{0}\left(\dot{\cup}_{n \geq 0} A_{n}\right)=\sum_{n \geq 0} \mu_{0}\left(A_{n}\right)$.

Those definitions of probability, finite, $\sigma$-finite and semifinite measures extend naturally to premeasures.

Theorem 2.1.10 (Caratheodory's extension theorem - creating measures from pre-measures). Let $X$ be a set, $\mathcal{A}$ be an algebra on $X$ and $\mu_{0}: \mathcal{A} \rightarrow[0, \infty]$ a pre-measure on $(X, \mathcal{A})$.

Use proposition 2.1.7, facing $\mathcal{A}$ as the family of "simple" sets, $\mathcal{S}:=\mathcal{A}$, and $\mu_{0}$ as a pre-outer measure, $\mu^{0}:=\mu_{0}$, to obtain the outer measure $\mu^{*}: \mathcal{P}(X) \rightarrow[0, \infty]$ on $X$ induced by $\mu^{0}=\mu_{0}$ (in general, proposition 2.1 .7 will not guarantee $\mu^{*}$ extends $\left.\mu^{0}\right)$.

Use Caratheodory's theorem, to obtain a complete measure $\hat{\mu}:=\left.\mu^{*}\right|_{\sigma\left(\mu^{*}\right)}$ in $\sigma\left(\mu^{*}\right)$ (obviously $\hat{\mu}$ coincides with $\mu^{*}$ where it is defined).

The present theorem adds that, when starting from a pre-measure, as herein assumed:

['eoe' and 'uoe' for existence and uniqueness of extension]

(eoe, i) $\left.\mu^{*}\right|_{\mathcal{A}}=\mu_{0}$, i.e., $\mu^{*}$ extends $\mu^{0}=\mu_{0}$.

(eoe, ii) $\mathcal{A} \subset \sigma\left(\mu^{*}\right)$, therefore $\hat{\mu}$ is a measure defined on a $\sigma$-algebra containing $\mathcal{A}$ which extends $\mu_{0}$, i.e., $\hat{\mu}(A)=\mu_{0}(A), \forall A \in \mathcal{A}$.

(eoe, iii) naturally $\sigma(\mathcal{A}) \subset \sigma\left(\mu^{*}\right)$, therefore $\mu:=\left.\hat{\mu}\right|_{\sigma(\mathcal{A})}=\left.\mu^{*}\right|_{\sigma(\mathcal{A})}$ is a measure defined on the $\sigma$-algebra generated by $\mathcal{A}$ which extends $\mu_{0}$.

(uoe, iv) if $\nu: \sigma(\mathcal{A}) \rightarrow[0, \infty]$ is another measure extending $\mu_{0}$, then $\nu \leq \mu$, i.e., $\nu(A) \leq$ $\mu(A), \forall A \in \sigma(\mathcal{A})$. The equality holds for those $A \in \sigma(\mathcal{A})$ such that $\mu(A)=\mu^{*}(A)<\infty$.

(uoe, $v$ ) if $\mu_{0}$ is $\sigma$-finite and $\nu: \sigma(\mathcal{A}) \rightarrow[0, \infty]$ is another measure extending $\mu_{0}$, then $\nu=\mu$.

(vi) if $\mu_{0}$ is $\sigma$-finite, then $\left(X, \sigma\left(\mu^{*}\right), \hat{\mu}\right)$ equals $(X, \overline{\sigma(\mathcal{A})}, \bar{\mu})$, the completion of $(X, \sigma(\mathcal{A}), \mu)$.

We are now ready to define the Lesbegue-Stieltjes measures on $\mathbb{R}$, a major class of Borelian measures on $\mathbb{R}$ (in fact, every Borelian measure which is finite on bounded measurable sets is a Lesbegue-Stieltjes measure and vice-versa). 
As a motivation, start with a Borelian measure on $\mathbb{R}$ which is finite on bounded measurable sets and define its centralized distribution function $F: \mathbb{R} \rightarrow \mathbb{R}$ given by

$$
F(x)= \begin{cases}\mu((0, x]) & x>0 \\ 0 & x=0 \\ -\mu((x, 0]) & x<0 .\end{cases}
$$

Using proposition 2.1.3, it follows that $F$ is non-decreasing, right-continuous and satisfies (a) $\mu((x, y] \cap \mathbb{R})=F(y)-F(x)$, for $x, y \in[-\infty, \infty], x \leq y,(\mathrm{~b}) F(\infty):=\lim _{x \rightarrow \infty} F(x) \in[0, \infty]$ and $(\mathrm{c})$ $F(-\infty):=\lim _{x \rightarrow-\infty} F(x) \in[-\infty, 0]$. Next, starting with a non-decreasing right-continuous function $F$, we construct a measure $\mu$ whose centered distribution is $F$.

The family of "simple" sets to which we will assign a prototypical measure is the family $\mathcal{S}=$ $\{(a, b] \cap \mathbb{R}: a, b \in[-\infty, \infty], a \leq b\}$ of h-intervals. It has the following convenient property.

Proposition 2.1.11 (Properties of the set of h-intervals). The family $\mathcal{S}$ of h-intervals introduced above is an elementary family, i.e., (i) $\varnothing \in \mathcal{S}$, (ii) $S, T \in \mathcal{S} \Rightarrow S \cap T \in \mathcal{S}$ and (iii) $S \in \mathcal{S} \Rightarrow S^{\mathrm{c}}$ is a finite union of disjoint elements of $\mathcal{S}$ (in this case, two elements).

Moreover, if $\mathcal{E}$ is an elementary family, then the collection $\mathcal{D}(\mathcal{E})$ of finite disjoint unions of elements in $\mathcal{E}$ is an algebra. In particular, $\mathcal{A}(\mathcal{E})=\mathcal{D}(\mathcal{E})$.

Finally, since $\sigma(\mathcal{S})=\mathcal{B}_{\mathbb{R}}, \sigma(\mathcal{D}(\mathcal{S}))=\mathcal{B}_{\mathbb{R}}$.

Is is now possible to define a Lesbegue-Stieltjes pre-measure.

Proposition 2.1.12 (Lesbegue-Stieltjes pre-measure). Let $F: \mathbb{R} \rightarrow \mathbb{R}$ be non-decreasing and rightcontinuous, $F(\infty):=\lim _{x \rightarrow \infty} F(x) \in[0, \infty]$ and $F(-\infty):=\lim _{x \rightarrow-\infty} F(x) \in[-\infty, 0]$. Define on $\mathcal{D}(\mathcal{S})$ the function $\mu_{0}$, assigning $\mu_{0}\left(\dot{\cup}_{j=0}^{n}\left(a_{j}, b_{j}\right] \cap \mathbb{R}\right)=\sum_{j=0}^{n}\left[F\left(b_{j}\right)-F\left(a_{j}\right)\right]$. Then $\mu_{0}$ is a pre-measure on the algebra $\mathcal{D}(\mathcal{S})=\mathcal{A}(\mathcal{S})$.

Now Caratheodory's extension theorem is used to obtain the Lesbegue-Stieltjes measure.

Theorem 2.1.13 (Lesbegue-Stieltjes measure). Let $F: \mathbb{R} \rightarrow \mathbb{R}$ be non-decreasing and rightcontinuous, with $F(\infty):=\lim _{x \rightarrow \infty} F(x) \in[0, \infty]$ and $F(-\infty):=\lim _{x \rightarrow-\infty} F(x) \in[-\infty, 0]$.

Then, using Caratheodory's extension theorem and its notation, there exists a unique ( $\mu_{0}$ is $\sigma$-finite) Borelian measure $\mu_{F}$ on $\left(\mathbb{R}, \mathcal{B}_{\mathbb{R}}\right)$, and an associated completion $\overline{\mu_{F}}$ on $\left(\mathbb{R}, \sigma\left(\mu^{*}\right)\right)$, whose centered distribution is $F$. In particular, $\mu_{F}((a, b] \cap \mathbb{R})=F(a)-F(b)$, for $a, b \in[-\infty, \infty], a \leq b(s o$ $\mu_{F}$ is finite on bounded measurable sets and hence on compact sets).

Let $G: \mathbb{R} \rightarrow \mathbb{R}$ be another non-decreasing and right-continuous function: then $\mu_{G}=\mu_{F} \Leftrightarrow F-G$ is constant.

Reciprocally, if $\mu$ on $\left(\mathbb{R}, \mathcal{B}_{\mathbb{R}}\right)$ is finite on bounded measurable sets (or on compact sets), then there exists $F: \mathbb{R} \rightarrow \mathbb{R}$ non-decreasing and right-continuous (namely, take $F$ as $\mu$ 's centered distribution) such that $\mu=\mu_{F}$.

We call the Lesbegue-Stieltjes measure associated to $F$ either $\mu_{F}$ or $\overline{\mu_{F}}$, which we may, by abuse of notation, jointly denote by $\mu_{F}$. When $\mu$ is a finite measure on $\mathbb{R}$, its cumulative distribution, $G$ : $\mathbb{R} \rightarrow \mathbb{R}$ given by $G(x)=\mu((-\infty, x])$, is non-decreasing, right-continuous and satisfies $(\mathrm{a}) \mu((x, y] \cap$ $\mathbb{R})=F(y)-F(x)$, for $x, y \in[-\infty, \infty], x \leq y,(\mathrm{~b}) G(\infty):=\lim _{x \rightarrow \infty} G(x) \in[0, \infty]$ and $(\mathrm{c}) G(-\infty):=0$. In this case, by the previous theorem, $G$ and $F$ differ by a constant, namely $\mu((-\infty, 0])$.

Our last assertion about Lesbegue-Stieltjes measures is a collection of their regularity properties.

Proposition 2.1.14 (Regularity of Lesbegue-Stieltjes measures). Let $\mu$ be a complete LesbegueStieltjes measure on $\mathbb{R}$ associated with $F: \mathbb{R} \rightarrow \mathbb{R}$ be non-decreasing and right-continuous, with $F(\infty):=\lim _{x \rightarrow \infty} F(x) \in[0, \infty]$ and $F(-\infty):=\lim _{x \rightarrow-\infty} F(x) \in[-\infty, 0]$. Let $\mathcal{M}_{\mu}$ be the domain of $\mu$. Then, $\forall E \in \mathcal{M}_{\mu}$ :

(i) $\mu(E)=\inf \left\{\sum_{j=0}^{\infty} \mu\left(\left(a_{j}, b_{j}\right] \cap \mathbb{R}\right): E \subset \cup_{j=0}^{\infty}\left(a_{j}, b_{j}\right] \cap \mathbb{R}, a_{j}, b_{j} \in[-\infty, \infty], a_{j} \leq b_{j}\right\}$ (immediate from the definition of $\mu^{*}$ induced from the pre (outer) measure defined in proposition 2.1.12). 
(ii) $\mu(E)=\inf \left\{\sum_{j=0}^{\infty}\left[F\left(b_{j}\right)-F\left(a_{j}\right)\right]: E \subset \cup_{j=0}^{\infty}\left(a_{j}, b_{j}\right] \cap \mathbb{R}, a_{j}, b_{j} \in[-\infty, \infty], a_{j} \leq b_{j}\right\}$ (immediate from $\mu$ being the Lesbegue-Stieltjes associated to $F)$.

(iii) $\mu(E)=\inf \left\{\sum_{j=0}^{\infty} \mu\left(\left(a_{j}, b_{j}\right) \cap \mathbb{R}\right): E \subset \cup_{j=0}^{\infty}\left(a_{j}, b_{j}\right) \cap \mathbb{R}, a_{j}, b_{j} \in[-\infty, \infty], a_{j} \leq b_{j}\right\}$ (a version of outer regularity)

(iv) $\mu(E)=\inf \{\mu(U): U$ open, $E \subset U \subset \mathbb{R}\}$ (outer regularity)

(v) $\mu(E)=\sup \{\mu(K): K$ compact, $K \subset E \subset \mathbb{R}\}$ (inner regularity)

(vi) $E=V \backslash N_{1}$, where $V \in G_{\delta}(\mathbb{R})$ (the set of countable intersections of open sets of $\mathbb{R}$ ) and $\mu\left(N_{1}\right)=0$.

(vii) $E=H \cup N_{2}$, where $H \in F_{\sigma}(\mathbb{R})$ (the set of countable unions of closed sets of $\mathbb{R}$ ) and $\mu\left(N_{2}\right)=0$.

(viii) such that $\mu(E)<\infty$, it holds that $\forall \epsilon>0, \exists A \subset \mathbb{R}$ a finite union of open intervals such that $\mu(E \triangle A)<\epsilon$ (a version of proposition 2.1.4).

We then focus on the most special Lesbegue-Stieltjes measure. It is the so-called Lesbegue measure, denoted by $m$, associated with $F=i d_{\mathbb{R}}$. As in proposition 2.1 .13 , by abuse of notation, $m$ refers to the measure of both $\left(\mathbb{R}, \mathcal{B}_{\mathbb{R}}, \mu_{i d}\right)$ and its completion $\left(\mathbb{R}, \overline{\mathcal{B}_{\mathbb{R}}}, \overline{\mu_{i d}}\right)$. The former $\sigma$-algebra, $\overline{\mathcal{B}_{\mathbb{R}}}$, characterized in propositions 2.1 .13 and 2.1 .10, is by denoted $\mathcal{L}$. In fact, $\mathcal{B}_{\mathbb{R}} \mp \mathcal{L} \mp \mathcal{P}(X)$, but we will not give a hint why. The next statement shows that the Lesbegue measure is algebraically well-behaved.

Proposition 2.1.15 (Lesbegue measure behaves well with translations and dilations). If $E \in \mathcal{L}$, then, for $s, r \in \mathbb{R}, s+E=\{s+x: x \in E\} \in \mathcal{L}, r E=\{r x: x \in E\} \in \mathcal{L}, m(s+E)=m(E)$ and $m(r E)=|r| m(E)$.

We now start to shift our review towards the integration theory based on such measures. Later, we will generalize the concept of measures by broadening its codomain.

By definition, a measurable function between measurable spaces $(X, \mathcal{A})$ and $(Y, \mathcal{B})$ (or on $(X, \mathcal{A})$ if the codomain is implicit), $f:(X, \mathcal{A}) \rightarrow(Y, \mathcal{B})$, satisfies $f^{-1}(B) \in \mathcal{A}, \forall B \in \mathcal{B}$. In practice, this property can be verified as follows.

Proposition 2.1.16 (Checking measurability in a generating algebra). Let $f$ be a function between measurable spaces $(X, \mathcal{A})$ and $(Y, \mathcal{B})$, with $\mathcal{B}$ generated by an algebra $\mathcal{G}$, i.e., $\sigma(\mathcal{G})=\mathcal{B}$. If $f^{-1}(B) \epsilon$ $\mathcal{A}, \forall B \in \mathcal{G}$, then $f$ is measurable.

The argument behind this proposition is pretty standard. Let $\mathcal{H}=\left\{B \subset Y: f^{-1}(B) \in \mathcal{A}\right\}$. Note that $\mathcal{H}$ is itself a $\sigma$-algebra and $\mathcal{G} \subset \mathcal{H}$, hence $\mathcal{B}=\sigma(\mathcal{G}) \subset \mathcal{H}$. As a consequence, $f$ is measurable.

By definition, a measure preserving function between measure spaces $(X, \mathcal{A}, \mu)$ and $(Y, \mathcal{B}, \nu)$, $f:(X, \mathcal{A}) \rightarrow(Y, \mathcal{B})$, is a measurable function such that $\nu(B)=\mu\left(f^{-1}(B)\right), \forall B \in \mathcal{B}$. In practice, this property can be verified as follows.

Proposition 2.1.17 (Checking measure preservation in a generating algebra). Let $f$ be a measurable function between measure spaces $(X, \mathcal{A}, \mu)$ and $(Y, \mathcal{B}, \nu)$, with $\mathcal{B}$ generated by an algebra $\mathcal{G}$, i.e., $\sigma(\mathcal{G})=\mathcal{B}$. If $\mu\left(f^{-1}(B)\right)=\nu(B), \forall B \in \mathcal{G}$, then $f$ is measure preserving.

The argument behind the proposition is precisely like the last one. Let $\mathcal{H}=\left\{B \subset Y: \mu\left(f^{-1}(B)\right)=\right.$ $\nu(B)\}$. Note that $\mathcal{H}$ is itself a $\sigma$-algebra and $\mathcal{G} \subset \mathcal{H}$, hence $\mathcal{B}=\sigma(\mathcal{G}) \subset \mathcal{H}$. As a consequence, $f$ is measure preserving.

Two measurable spaces $(X, \mathcal{A})$ and $(Y, \mathcal{B})$ are said to be isomorphic if there exists a measurable bijection $h: X \rightarrow Y$, admitting a measurable inverse.

Two measure spaces $(X, \mathcal{A}, \mu)$ and $(Y, \mathcal{B}, \nu)$ are said to be strictly isomorphic if there exists a measure-preserving bijection $h: X \rightarrow Y$, admitting a measurable inverse (which will be measure preserving). They are almost isomorphic if, in each, there are subsets $X_{0} \subset X$ and $Y_{0} \subset Y$ of full measure so that the corresponding restricted measure spaces are strictly isomorphic. Given a measure space $(X, \mathcal{A}, \mu)$ and $X_{0} \subset X$, the restricted measure space is $\left(X_{0},\left.\mathcal{A}\right|_{X_{0}},\left.\mu\right|_{\left.\mathcal{A}\right|_{X_{0}}}\right)$, where $\left.\mathcal{A}\right|_{X_{0}}=\left\{A \cap X_{0}: A \in \mathcal{A}\right\}$ and $\left.\mu\right|_{\left.\mathcal{A}\right|_{X_{0}}}$ is a standard function restriction. 
Obviously, the composition of measurable (measure preserving) functions is a measurable (measure preserving) function.

Proposition 2.1.18 (Measurable functions under elementary and limiting operations are measurable). If $f, g: X \rightarrow K, K \in\{\mathbb{C},[-\infty, \infty]\}$, are measurable, then $f+g, f g$ and $f / g$ (when defined) are measurable. Additionally, if $h_{j}: X \rightarrow[-\infty, \infty], j \geq 0$, are measurable, then $a(x)=\sup _{j} h_{j}(x), b(x)=$ $\inf _{j} h_{j}(x), c(x)=\limsup _{j} h_{j}(x), d(x)=\liminf _{j} h_{j}(x)$ are measurable. In particular, max $\left\{h_{1}, h_{2}\right\}$, $\min \left\{h_{1}, h_{2}\right\},\left|h_{1}\right|$ and $\lim _{j} h_{j}$ (if defined for all $x \in X$ ) are measurable.

Also, continuous functions are measurable functions with respect to the Borelian $\sigma$-algebra on the domain and codomain, since we can check the preimage property for the generating collection of open sets.

A simple function on $(X, \mathcal{A}), \phi: X \rightarrow \mathbb{C}$ (or $\mathbb{R}$ ), is a finite linear combination of characteristic functions of measurable sets using complex (or real) coefficients. It can be equivalently expressed by different linear combinations, even though we fix a standard representation: since the image of a simple function is finite (while a measurable function with finite image is, conversely, simple), we let $E_{j}=f^{-1}\left(\left\{z_{j}\right\}\right)$, where $\operatorname{Img}(f)=\left\{z_{0}, \ldots, z_{n}\right\}$, and $\phi=\sum_{j=0}^{n} z_{j} \mathcal{X}_{E_{j}}$. As a sum of measurable functions, a simple function is also measurable.

First, we assign integrals to simple functions on $(X, \mathcal{A})$ taking values in $[0, \infty]$. Let $\phi=$ $\sum_{j=0}^{n} a_{j} \mathcal{X}_{E_{j}}\left(a_{j} \geq 0, j \geq 0\right)$, be a simple function on $(X, \mathcal{A})$ in standard representation and let $\mu$ be a measure on $X$. We define the integral of $\phi$ with respect to $\mu$ by $\int \phi d \mu:=\sum_{j=0}^{n} a_{j} \mu\left(E_{j}\right) \in[0, \infty]$ (remember $0 \cdot \infty=0$ ). This integral is homogeneous of degree one (taking scalars $c \in[0, \infty)$ ), additive and monotonic. We also write $\int \phi d \mu=\int \phi=\int \phi(x) d \mu(x)=\int \phi(x) \mu(d x), \int_{A} \phi d \mu=\int \phi \mathcal{X}_{A} d \mu$ and $\int=\int_{X}$. This notation can be extended naturally to all integrals we will define later and will not be repeated.

Second, we assign integrals to measurable functions on $(X, \mathcal{A})$ taking values in $[0, \infty]$ (these functions comprise the set $\left.L^{+}(\mathrm{X})\right)$. Let $f$ be in $L^{+}(X)$ and $\mu$ be a measure on $X$. We define $\int f d \mu:=\sup \left\{\int \phi d \mu: \phi\right.$ simple function on $\left.(X, \mathcal{A}), 0 \leq \phi \leq f\right\}$. By the previous monotonicity, this definition extends the former. If $f$ has finite integral we call it $(\mu$-)integrable, while if it has infinite integral we call it $(\mu-)$ quasi-integrable.

Proposition 2.1.19 (Integration is indifferent to changes in sets of measure zero, v1). Let $f$ be in $L^{+}(X)$ and $\mu$ a measure on $X$. Then $\int f d \mu=0$ if, and only if, $f=0 \mu$-a.e.

Additionally, this integral is homogeneous of degree one (taking scalars $c \in[0, \infty)$ ) and monotonic. It is also additive, as a consequence of the next two results.

Proposition 2.1.20 (Measurable Functions are approximated by simple functions). Let $(X, \mathcal{A})$ be a measurable space and $f: X \rightarrow[0, \infty]$ (or $\mathbb{C}$ ). Then there exists a sequence $\left(\phi_{n}\right)_{n \geq 0}$ of positive (complex) valued simple functions on $(X, \mathcal{A})$ such that $0 \leq\left|\phi_{1}\right| \leq \ldots \leq\left|\phi_{n}\right| \leq \ldots \leq|f|, \phi_{n} \rightarrow f$ pointwise and uniformly on any set on which $f$ is bounded.

The following theorem is the first major convergence theorem. Together with the last proposition and monotonicity, it also permits to write the integral of $f \in L^{+}(X)$ as a limit of integrals of simple functions increasingly converging to $f$ instead of the original supremum.

Theorem 2.1.21 (Monotone Convergence Theorem - a.e. version). Let $(X, \mathcal{A}, \mu)$ be a measure space. If $\left(f_{n}\right)_{n \geq 0}$ is a sequence in $L^{+}(X)$ such that $f_{n}(x)$ \ $f(x)$, $\mu$-a.e. $x \in X$, then $\int f d \mu=$ $\int \lim _{n} f_{n} d \mu=\lim _{n} \int f_{n} d \mu$.

Hence, under the same hypothesis, it follows that $\int \sum_{n} f_{n} d \mu=\sum_{n} \int f_{n} d \mu$.

The second major converge theorem is the following.

Theorem 2.1.22 (Fatou's Lemma - a.e. version). Let $(X, \mathcal{A}, \mu)$ be a measure space. If $\left(f_{n}\right)_{n \geq 0}$ is a sequence in $L^{+}(X)$, then $\int \liminf _{n} f_{n} d \mu \leq \liminf _{n} \int f_{n} d \mu$. 
We will now extend the domain of functions to which we (try to) assign integrals to those taking values in $[-\infty, \infty]$ and $\mathbb{C}$.

Let $(X, \mathcal{A}, \mu)$ be a measure space, $f: X \rightarrow[-\infty, \infty]$ be a measurable function and $f^{+}, f^{-}$: $X \rightarrow[-\infty, \infty]$ be given, respectively, by $f^{+}(x)=\max \{f(x), 0\}$ and $f^{-}(x)=\max \{-f(x), 0\}$, so that $f=f^{+}-f^{-}$and $|f|=f^{+}+f^{-}$. When $\int f^{+} d \mu$ and $\int f^{-} d \mu$ are not both infinite, we define the integral of $f$ with respect to $\mu$ to be $\int f d \mu:=\int f^{+} d \mu-\int f^{-} d \mu \in[-\infty, \infty]$. If $f$ has finite integral we call it $(\mu$-)integrable, while if it has infinite integral we call it $(\mu-)$ quasi-integrable. Now, not every measurable function will be one of them, i.e., not every measurable function will be assigned an integral, as when $\int f^{+} d \mu$ and $\int f^{-} d \mu$ are both infinite. Clearly, $f$ is integrable if, and only if, $|f|$ is integrable.

Let $(X, \mathcal{A}, \mu)$ be a measure space and $f: X \rightarrow \mathbb{C}$ be a measurable function. When $\int \operatorname{Re}(f) d \mu$ and $\int \operatorname{Im}(f) d \mu$ are both finite $(\operatorname{Re}(f)$ and $\operatorname{Im}(f)$ are measurable), we define the integral of $f$ with respect to $\mu$ to be $\int f d \mu:=\int \operatorname{Re}(f) d \mu+i \int \operatorname{Im}(f) d \mu \in \mathbb{C}$. Here $(\mu$-)integrable functions are exactly those measurable functions to which we assign integrals. The concept of $(\mu$-)quasi-integrable functions is not applicable anymore, as now the integral is complex-valued.

We highlight the following properties.

Proposition 2.1.23 (The set of integrable functions is a vector space). The set of real (complex) valued integrable functions is a real (complex) vector space and the integral is a linear functional on it.

Proposition 2.1.24 (A triangular inequality, v1). Let $(X, \mathcal{A}, \mu)$ be a measure space. If $f$ is ( $\mu$ )integrable (taking values in $[0, \infty],[-\infty, \infty]$ or $\mathbb{C}$ ), then $\left|\int f d \mu\right| \leq \int|f| d \mu$.

Proposition 2.1.25 (Integration is indifferent to changes in sets of measure zero, v2). Let $(X, \mathcal{A}, \mu)$ be a measure space. If $f$ and $g$ are ( $\mu$-)integrable (taking values in $[0, \infty],[-\infty, \infty]$ or $\mathbb{C}$ ), then $\int_{E} f d \mu=\int_{E} g d \mu, \forall E \in \mathcal{B}$ if, and only if, $\int|f-g| d \mu=0$ if, and only if, $f=g, \mu-a . e$. .

Finally, we state the last major convergence theorem.

Theorem 2.1.26 (Dominated convergence theorem). Let $(X, \mathcal{A}, \mu)$ be a measure space. If $\left(f_{n}\right)_{n \geq 0}$ is a sequence of ( $\mu$-)integrable functions (taking values in $[0, \infty],[-\infty, \infty]$ or $\mathbb{C}$ ) such that $f_{n} \rightarrow f$ $\mu$-a.e. and there exists an integrable function $g$ (taking values in $[0, \infty]$ ), with $\left|f_{n}\right| \leq g \mu$-a.e. $\forall n \geq 0$, then $f$ is integrable and $\int f d \mu=\int \lim _{n} f_{n} d \mu=\lim _{n} \int f_{n} d \mu$.

Hence, considering $\left(f_{n}\right)_{n \geq 0}$ as before and $g=\sum_{n}\left|f_{n}\right|$. If $\int \sum_{n}\left|f_{n}\right| d \mu=\sum_{n} \int\left|f_{n}\right| d \mu$ (by monotone convergence theorem) is finite, then $\sum_{n} f_{n}$ converges $\mu$-a.e. to an integrable function $f$ and $\int f d \mu=$ $\int \sum_{n} f_{n} d \mu=\sum_{n} \int f_{n} d \mu$.

We now shift our review towards the theory of $L^{p}$ spaces. During the exposition of this subject, consider a fixed measure space $(X, \mathcal{A}, \mu)$.

Let $f$ be a $\mathbb{C}$-valued measurable function. For $p \in(0, \infty)$, define $\|f\|_{p}:=\left(\int|f|^{p} d \mu\right)^{1 / p} \in[0, \infty]$; for $p=\infty$, define $\|f\|_{\infty}=\inf \{C \in[0, \infty]:|f| \leq C \mu$-a.e. $\} \in[0, \infty]$ (which adapts the uniform norm to the present setting). Now, for $p \in(0, \infty]$, define $\mathcal{L}^{p}(\mu, \mathbb{C}):=\left\{f: X \rightarrow \mathbb{C} ; f\right.$ is measurable and $\left.\|f\|_{p}<\infty\right\}$. Note that $\mathcal{L}^{1}(\mu, \mathbb{C})$ is simply the set of $\mathbb{C}$-valued integrable functions.

With pointwise addition and multiplication by scalars, the set $\mathcal{L}^{1}(\mu, \mathbb{C})$ is a complex linear subspace of $\{f: X \rightarrow \mathbb{C}\}$ and $\int \cdot d \mu: \mathcal{L}^{1}(\mu, \mathbb{C}) \rightarrow \mathbb{C}$ is a linear functional.

We could want to consider measurable functions taking values in $[-\infty, \infty]$ or $[0, \infty]$. The problem is that sums and differences of functions in those sets are not well defined. Nonetheless, every definition in the next-to-last paragraph, not involving sums, can be directly adapted, by changing "C্C" to " $[-\infty, \infty]$ " or " $[0, \infty]$ ".

Moreover, for $K \in\{[-\infty, \infty],[0, \infty]\}$, every $K$-valued measurable measure function $f$ such that $f^{-1}(\{-\infty\})$ and $f^{-1}(\{\infty\})$ have null measure, in particular for every $f \in \mathcal{L}^{p}(\mu, K)$ (in the aforementioned adapted sense), we can consider the $K$-valued measurable measure function $\hat{f}=f \mathcal{X}_{f^{-1}(\mathbb{R})}$, whose image is in $\mathbb{R}$ and such that $\hat{f}=f, \mu$-a.e.. Every following result can be extended to 
$K \in\{[-\infty, \infty],[0, \infty]\}$ in the sense of considering $\hat{f}$ instead of $f$ and collecting the associated findings. As such, every following result is presented for $K=\mathbb{C}$.

A pair $p, q \in[1, \infty]$ is said to be a pair of conjugate exponents if $p^{-1}+q^{-1}=1$ (here 1 and $\infty$ are conjugate exponents).

Proposition 2.1.27 (Hölder inequality). Let $f, g: X \rightarrow \mathbb{C}$, be measurable functions and $p, q, r \in$ $[1, \infty]$ be such that $1 / p+1 / q=1 / r$. Then $\|f g\|_{r} \leq\|f\|_{p}\|g\|_{q}$. Note we are not stating, a priori, that $f$, $g$ or fg are in $\mathcal{L}^{p}(\mu, \mathbb{C}), \mathcal{L}^{q}(\mu, \mathbb{C})$ or $\mathcal{L}^{r}(\mu, \mathbb{C})$.

Proposition 2.1.28 (Minkowski inequality). Let $f, g: X \rightarrow \mathbb{C}$, be measurable functions and $p \in$ $[1, \infty)$. Then $\|f+g\|_{p} \leq\|f\|_{p}+\|g\|_{p}$. Note we are not stating, a priori, that $f, g$ or $f+g$ are in $\mathcal{L}^{p}(\mu, \mathbb{C})$.

From Minkowski inequality, for $p \in[1, \infty], \mathcal{L}^{p}(\mu, \mathbb{C})$ is a vector space and $\|\cdot\|_{p}$ is an associated seminorm (which can assume value 0 at not identically 0 functions $f$, as long as $f=0 \mu$-a.e.).

For each $p \in[1, \infty]$, we define an equivalence relation on $\mathcal{L}^{p}(\mu, \mathbb{C})$, by stating $f \sim g$ whenever $\|f-g\|_{p}=0$, i.e., $f=g \mu$-a.e.. So we obtain $\mathcal{N}:=\left\{f \in \mathcal{L}^{p}(\mu, \mathbb{C}):\|f\|_{p}=0\right\}$, a complex linear subspace of $\mathcal{L}^{p}(\mu, \mathbb{C})$. Now, we define the set of equivalence classes on $\mathcal{L}^{p}(\mu, \mathbb{C}), \mathscr{L}^{p}(\mu, \mathbb{C}):=\mathcal{L}^{p}(\mu, \mathbb{C}) / \mathcal{N}=$ $\left\{[f]: f \in \mathcal{L}^{p}(\mu, \mathbb{C})\right\}$, where $[f]:=\left\{g \in \mathcal{L}^{p}(\mu, \mathbb{C}): f \sim g\right.$, i.e., $\left.f-g \in \mathcal{N}\right\}$ is called the equivalence class of $f$.

Adapting vector addition, multiplication by scalars and $\|\cdot\|_{p}$ to $\mathscr{L}^{p}(\mu, \mathbb{C})$, by using representatives of each equivalence class and performing the previously defined operations (these definitions will be well-defined), we have:

Proposition 2.1.29 ( $\mathscr{L}^{p}$ is a Banach space). For $p \in[1, \infty],\left(\mathscr{L}^{p}(\mu, \mathbb{C}),\|\cdot\|_{p}\right)$ is a Banach space (complete normed vector space).

As a normed space, it has an associated notion of convergence: given $\left(\left[f_{n}\right]\right)_{n \geq 0}$ and $[f]$, all of them in $\mathscr{L}^{p}(\mu, \mathbb{C}),\left(\left[f_{n}\right]\right)_{n \geq 0}$ is said to converge to $f$ in $\mathscr{L}^{p}$ whenever $\left\|\left[f_{n}\right]-[f]\right\|_{p} \rightarrow 0$. This same notion of convergence allows the following result.

Proposition 2.1.30 (Simple functions are dense in $\left.\mathscr{L}^{p}\right)$. For $p \in[1, \infty]$, the set of simple functions $S:=\left\{[\phi] \in \mathscr{L}^{p}(\mu, \mathbb{C}): \phi=\sum_{n=0}^{k} a_{n} \mathcal{X}_{E_{n}}\right.$ is simple with $a_{n} \in \mathbb{C}$ and $\mu\left(E_{n}\right)<\infty$, for $\left.n=1, \ldots, k\right\}$ is dense in $\mathscr{L}^{p}(\mu, \mathbb{C})$.

Borrowing the norm just defined on $\mathscr{L}^{p}(\mu, \mathbb{C})$, given $\left(f_{n}\right)_{n \geq 0}$ and $f$, all of them in $\mathcal{L}^{p}(\mu, \mathbb{C})$, $\left(f_{n}\right)_{n \geq 0}$ is said to converge to $f$ in $\mathcal{L}^{p}$ whenever $\left\|f_{n}-f\right\|_{p} \rightarrow 0$.

From now on, an abuse of notation will be allowed by denoting both $\mathcal{L}^{p}(\mu, \mathbb{C})$ and $\mathscr{L}^{p}(\mu, \mathbb{C})$ by $L^{p}(\mu, \mathbb{C})$, relying on the context to make the meaning clear. In particular, we may use $L^{p}$ convergence in order to refer to either $\mathcal{L}^{p}$ or $\mathscr{L}^{p}$ convergences. From an element of $\mathcal{L}^{p}(\mu, \mathbb{C})$, the reader can always obtain an element of $\mathscr{L}^{p}(\mu, \mathbb{C})$, by taking its equivalence class.

We conclude the review on $L^{p}$ spaces with the following three propositions. The first of them is a direct application of dominated convergence theorem.

Proposition 2.1.31 (Almost everywhere convergence and $L^{p}$ convergence). Let $p \in[1, \infty), f_{n}$, $n \geq 0$, and $f$ be $\mathbb{C}$-valued measurable functions. If $\exists g \in L^{p}(\mu, \mathbb{C})$ such that $\left|f_{n}\right| \leq g, \mu$-a.e., $\forall n \geq 0$, and $f_{n} \rightarrow f$, $\mu$-a.e., then $\left\|f_{n}-f\right\|_{p} \rightarrow 0$.

Proposition 2.1.32 (A function in $L^{p}$ is a sum of one from a higher exponent space and another from a lower). If $0<p<q<r \leq \infty$, then $L^{q}(\mu, \mathbb{C}) \subset L^{p}(\mu, \mathbb{C})+L^{r}(\mu, \mathbb{C})$.

Proposition 2.1.33 (The $L^{p}$ inclusion). If $\mu(X)<\infty$ and $0<p<q \leq \infty$, then $L^{q}(\mu, \mathbb{C}) \subset L^{p}(\mu, \mathbb{C})$ and $\|f\|_{p} \leq\|f\|_{q} \mu(X)^{1 / p-1 / q}$.

In the above proposition, if we relax the finiteness of $\mu$, we get the following simple counterexample: $X=[1, \infty], \mu=m, \int 1 / x d \mu=\infty$, but $\int 1 / x^{2} d \mu<\infty$.

We now shift our review towards some results about product measures. 
Let $\left(\left(X_{\alpha}, \mathcal{A}_{\alpha}\right)\right)_{\alpha \in \Lambda}$ be an arbitrary family of measurable spaces, $X$ be a set and $\left(f_{\alpha}: X \rightarrow\right.$ $\left.X_{\alpha}\right)_{\alpha \in \Lambda}$ be a family of functions. Then there exists the smallest $\sigma$-algebra $\mathcal{A}$ on $X$ that makes every $f_{\alpha}$ measurable, called the $\sigma$-algebra on $X$ induced by the family $\left(f_{\alpha}\right)_{\alpha \in \Lambda}: \mathcal{A}=\sigma(\mathcal{T})$, where $\mathcal{T}=\left\{V \subset X: \exists \alpha \in \Lambda\right.$ and $A \in \mathcal{B}_{\alpha}$ such that $\left.V=f_{\alpha}{ }^{-1}(A)\right\}$.

A consequence of the $\sigma$-algebra induced by the family $\left(f_{\alpha}\right)_{\alpha \in \Lambda}$ to the measurability of functions is not only that each $f_{\alpha}$ gets measurable, but also that:

Proposition 2.1.34 (Measurability of the composition with the inducing family). Let $X$ be $a$ set, $\left(X_{\alpha}, \mathcal{A}_{\alpha}\right)_{\alpha \in \Lambda}$ be a family of measurable spaces, $\left(f_{\alpha}: X \rightarrow X_{\alpha}\right)_{\alpha \in \Lambda}$ be a family of functions, $\mathcal{A}$ be the $\sigma$-algebra on $X$ induced by the family $\left(f_{\alpha}\right)_{\alpha \in \Lambda},(Y, \mathcal{B})$ be another measure space and $g:(Y, \mathcal{B}) \rightarrow(X, \mathcal{A})$ be a function.

Then $g$ is measurable $\Leftrightarrow f_{\alpha} \circ g:(Y, \mathcal{B}) \rightarrow\left(X_{\alpha}, \mathcal{A}_{\alpha}\right)$ is measurable, $\forall \alpha \in \Lambda$ (the $\Rightarrow$ implication is trivial, since the composition of measurable functions is measurable).

Let $\left(\left(X_{\alpha}, \mathcal{B}_{\alpha}\right)\right)_{\alpha \in \Lambda}$ be an arbitrary family of measurable spaces, $X$ be their cartesian product, $X=\prod_{\alpha \in \Lambda} X_{\alpha}$, and $\left(p r_{\alpha}: X \rightarrow X_{\alpha}\right)_{\alpha \in \Lambda}$ be the family of canonical projections. The $\sigma$-algebra on $X$ induced by the family $\left(p r_{\alpha}\right)_{\alpha \in \Lambda}$ is called the product $\sigma$-algebra (on the product space $X$ ) and denoted by $\otimes_{\alpha \in \Lambda} \mathcal{A}_{\alpha}$.

One should ask whether $\otimes_{\alpha \in \Lambda} \mathcal{A}_{\alpha}$ equals the $\sigma$-algebra generated by the set of "rectangles whose $\alpha$-components are measurable sets in $\mathcal{A}_{\alpha}$ ", i.e., whether it equals $\sigma(\mathcal{R})$, where $\mathcal{R}=\left\{\prod_{\alpha \in \Lambda} A_{\alpha} \subset\right.$ $\left.X: A_{\alpha} \in \mathcal{A}_{\alpha}, \alpha \in \Lambda\right\}$. It will be the case when $\Lambda$ is countable. This is because (i) in general, $\mathcal{T} \subset \mathcal{R}$, since $V \in \mathcal{T} \Rightarrow V=p r_{\alpha^{*}}{ }^{-1}(A)$ for some $\alpha^{*} \in \Lambda$ and $A \in \mathcal{A}_{\alpha^{*}} \Rightarrow V=\prod_{\alpha \in \Lambda} U_{\alpha}$, where $U_{\alpha}=A$ if $\alpha=\alpha^{*}$ and $U_{\alpha}=X_{\alpha}$ otherwise $\Rightarrow V \in \mathcal{R}$ and (ii) when $\Lambda$ is countable, $\mathcal{R} \subset \mathcal{T}$, since $V \in \mathcal{R} \Rightarrow V=\prod_{\alpha \in \Lambda} A_{\alpha}=\cap_{\alpha \in \Lambda} p r_{\alpha}{ }^{-1}\left(A_{\alpha}\right) \in S$, once each $p r_{\alpha}{ }^{-1}\left(A_{\alpha}\right) \in \bigotimes_{\alpha \in \Lambda} \mathcal{A}_{\alpha}$ and the intersection is countable. Moreover, if, for each $\alpha \in \Lambda, \mathcal{A}_{\alpha}$ is generated by a set $\mathcal{G}_{\alpha}$, a stronger version will hold: $\otimes_{\alpha \in \Lambda} \mathcal{A}_{\alpha}=\sigma\left(\mathcal{R}^{\prime}\right)$, where $\mathcal{R}^{\prime}=\left\{\prod_{\alpha \in \Lambda} G_{\alpha} \subset X: G_{\alpha} \in \mathcal{G}_{\alpha}, \alpha \in \Lambda\right\}$.

In the present context, the last proposition has a direct corollary: a function $g$ taking values in the product space is measurable if (and only if) its coordinate functions are also measurable.

The following result explores, when each $X_{\alpha}$ is a topological space equipped with its Borel $\sigma$ algebra $\mathcal{B}_{X_{\alpha}}$ and $X=\prod_{\alpha \in \Lambda} X_{\alpha}$ is equipped with the product topology, how $\otimes_{\alpha \in \Lambda} \mathcal{B}_{X_{\alpha}}$ compares to $\mathcal{B}_{X}$.

Proposition 2.1.35 (Product of Borel $\sigma$-algebras and Borel $\sigma$-algebra of the product topology). Let $\left(\left(X_{\alpha}, \tau_{\alpha}, \mathcal{B}_{X_{\alpha}}\right)\right)_{\alpha \in \Lambda}$ be a family of topological spaces each equipped with its Borel $\sigma$-algebra and $X=\prod_{\alpha \in \Lambda} X_{\alpha}$ equipped with the product topology $\tau$.

Then: (i) $\otimes_{\alpha \in \Lambda} \mathcal{B}_{X_{\alpha}} \subset \mathcal{B}_{X}$

(ii) $\otimes_{\alpha \in \Lambda} \mathcal{B}_{X_{\alpha}}=\mathcal{B}_{X}$ in the following cases: (a) $\forall \alpha \in \Lambda, \tau_{\alpha}$ has a countable basis (separable metric spaces are a special case) and all but a countable quantity of the $X_{\alpha}$ are singletons or, equivalently, (b) $\tau$ has a countable basis.

As a corollary, $\mathcal{B}_{\mathbb{R}^{e}}=\bigotimes_{j=0}^{e-1} \mathcal{B}_{\mathbb{R}}, e \in \mathbb{N} \cup\{\infty\}$, analogously with $\overline{\mathbb{R}}$ or $\mathbb{C}$ instead of $\mathbb{R}$

Now, starting with finite family of measure spaces $\left(\left(X_{j}, \mathcal{A}_{j}, \mu_{j}\right)\right)_{j=0}^{n}$, we wish to construct a product measure on the product measurable space $\left(\prod_{j=0}^{n} X_{j}, \otimes_{j=0}^{n} \mathcal{A}_{j}\right)$. With no loss of generality, we restrict for the case when $n=2$, and use the measure spaces $(X, \mathcal{A}, \mu)$ and $(Y, \mathcal{B}, \nu)$. For such, we assign a suitable prototypical (product) measure in the set $\mathcal{R}=\{A \times B \subset X \times Y: A \in \mathcal{A}, B \in \mathcal{B}\}$ of rectangles, adapt it to an algebra and leverage on Caratheodory's extension theorem.

In order to do so, we rephrase proposition 2.1.11 to the set of rectangles.

Proposition 2.1.36 (Properties of the set of rectangles). The family $\mathcal{R}$ of rectangles introduced above is an elementary family, i.e., (i) $\varnothing=\varnothing \times \varnothing \in \mathcal{R}$, (ii) $(A \times B),(C \times D) \in \mathcal{R} \Rightarrow(A \times B) \cap(C \times D)=$ $(A \cap C) \times(B \cap D) \in \mathcal{R}$ and (iii) $(A \times B) \in \mathcal{S} \Rightarrow(A \times B)^{\mathrm{c}}=\left(X \times B^{\mathrm{c}}\right) \cup\left(A^{\mathrm{c}} \times B\right)=\left(A^{\mathrm{c}} \times X\right) \cup\left(A \times B^{\mathrm{c}}\right)$ is a finite union of disjoint elements of $\mathcal{R}$ (in this case, two elements).

Moreover, if $\mathcal{E}$ is an elementary family, then the collection $\mathcal{D}(\mathcal{E})$ of finite disjoint union of elements in $\mathcal{E}$ is an algebra. In particular $\mathcal{A}(\mathcal{E})=\mathcal{D}(\mathcal{E})$.

Finally, since $\sigma(\mathcal{R})=\mathcal{A} \otimes \mathcal{B}, \sigma(\mathcal{A}(\mathcal{R}))=\mathcal{A} \otimes \mathcal{B}$. 
It is now possible to define a (product) pre-measure.

Proposition 2.1.37 (Product pre-measure). Let $(X, \mathcal{A}, \mu)$ and $(Y, \mathcal{B}, \nu)$ be two measure spaces, $E=\dot{\cup}_{j=0}^{n} A_{j} \times B_{j}$ be a disjoint union of rectangles in $\mathcal{R}$ and $(\mu \times \nu)_{0}: \mathcal{D}(\mathcal{R}) \rightarrow[0, \infty]$ be the set function assigning $E=\dot{\cup}_{j=0}^{n} A_{j} \times B_{j} \mapsto(\mu \times \nu)_{0}\left(\dot{\cup}_{j=0}^{n} A_{j} \times B_{j}\right)=\sum_{j=0}^{n} \mu\left(A_{j}\right) \nu\left(B_{j}\right)$. In special, if $A \times B$ is one such rectangle, $(\mu \times \nu)_{0}(A \times B)=\mu(A) \nu(B)$ and $(\mu \times \nu)_{0}\left(\dot{\cup}_{j=0}^{n} A_{j} \times B_{j}\right)=\sum_{j=0}^{n}(\mu \times \nu)_{0}\left(A_{j} \times B_{j}\right)$. Then $(\mu \times \nu)_{0}$ (is well defined, independent of the representation chosen for $E$ and) is a pre-measure on the algebra $\mathcal{D}(\mathcal{R})=\mathcal{A}(\mathcal{R})$.

Now Caratheodory's extension theorem is used to obtain the product measure.

Theorem 2.1.38 (Product measure). Let $(X, \mathcal{A}, \mu)$ and $(Y, \mathcal{B}, \nu)$ be two measure spaces.

Then, by Caratehordory's extension theorem, there exists a measure $\mu \times \nu$ on $\mathcal{A} \otimes \mathcal{B}$ extending $(\mu \times \nu)_{0}$, and an associated completion $\overline{\mu \times \nu}$ on $\left(X \times Y, \sigma\left((\mu \times \nu)^{*}\right)\right)$.

Also, if $\mu$ and $\nu$ are $\sigma$-finite, then $(\mu \times \nu)_{0}$ is $\sigma$-finite pre-measure. Therefore, the extension is unique. In particular, no other measure $\eta$ on $\mathcal{A} \otimes \mathcal{B}$ other than $\mu \times \nu$ is such that $\eta(A \times B)=$ $\mu(A) \nu(B), \forall A \in \mathcal{A}, B \in \mathcal{B}$ (because it will have to coincide with $(\mu \times \nu)_{0}$ on $\mathcal{D}(\mathcal{R})=\mathcal{A}(\mathcal{R})$ ).

An analogous construction can be done to define $\mu_{1} \times \ldots \times \mu_{n}$. But one can check that $\mathcal{A}_{1} \otimes \ldots \otimes \mathcal{A}_{n}=$ $\left(\mathcal{A}_{1} \otimes \ldots \otimes \mathcal{A}_{n-1}\right) \otimes \mathcal{A}_{n}$ and $\mu_{1} \times \ldots \times \mu_{n}=\left(\mu_{1} \times \ldots \times \mu_{n-1}\right) \times \mu_{n}$, so, with minor adaptations, it suffices to understand the case when $n=2$.

The product measure implies certain relations will hold between $\mu \times \nu$ integration in $X \times Y, \mu$ integration on $X$ and $\nu$ integration on $Y$. The following theorem summarizes these relations.

Theorem 2.1.39 (Fubini-Tonelli theorem). Let $(X, \mathcal{A}),(Y, \mathcal{B})$ and $(X \times Y, \mathcal{A} \otimes \mathcal{B})$ be measurable spaces and $\mu, \nu$ (and, consequently, $\mu \times \nu$ ) be $\sigma$-finite measures on those spaces, respectively.

(a.1) if $E \in \mathcal{A} \otimes \mathcal{B}$, then $E_{x}:=\{y \in Y:(x, y) \in E\} \in \mathcal{B}, \forall x \in X$ and $E^{y}:=\{x \in X:(x, y) \in E\} \in$ $\mathcal{A}, \forall y \in Y$. Also, if, $f:(X \times Y, \mathcal{A} \otimes \mathcal{B}) \rightarrow(Z, \mathcal{C})$ is measurable, then $f_{x}: y \in(Y, \mathcal{B}) \mapsto f(x, y) \in(Z, \mathcal{C})$ is measurable, $\forall x \in X$, and $f^{y}: x \in(X, \mathcal{A}) \mapsto f(x, y) \in(Z, \mathcal{C})$ is measurable, $\forall y \in Y$.

(a.2) if $E \in \mathcal{A} \otimes \mathcal{B}$, then the functions $x \in X \mapsto \nu\left(E_{x}\right) \in[0, \infty]$ and $y \in Y \mapsto \mu\left(E^{y}\right) \in[0, \infty]$ are measurable and $\mu \times \nu(E)=\int \nu\left(E_{x}\right) d \mu(x)=\int \mu\left(E^{y}\right) d \nu(y)$

(b) (Tonelli) if $f$ is in $L^{+}(X \times Y)$, then (b.i) $g: x \in X \mapsto \int f_{x}(y) d \nu(y) \in[0, \infty]$ and $h: y \in Y \mapsto$ $\int f^{y}(x) d \mu(x) \in[0, \infty]$ (well defined, by (a.1)) are in $L^{+}(X)$ and $L^{+}(Y)$, satisfying:

(b.ii) $[*] \int f d(\mu \times \nu)=\int g(x) d \mu(x)=\int\left[\int f_{x}(y) d \nu(y)\right] d \mu(x)=\int\left[\int f(x, y) d \nu(y)\right] d \mu(x)$, and

$\int f d(\mu \times \nu)=\int h(y) d \nu(y)=\int\left[\int f_{y}(x) d \mu(x)\right] d \nu(y)=\int\left[\int f(x, y) d \mu(x)\right] d \nu(y)$.

(c) (Fubini) if $f$ is in $L^{1}(\mu \times \nu)$, then (c.i) $f_{x}$ is in $L^{1}(\nu)$, for $\mu$-a.e.x $\in X$, and $f^{y}$ is in $L^{1}(\mu)$, for $\nu$-a.e.y $\in Y$, (c.ii) $g: x \in X \mapsto \int f_{x}(y) d \nu(y) \in[0, \infty]$ and $h: y \in Y \mapsto \int f^{y}(x) d \mu(x) \in[0, \infty]$ are, respectively, $\mu$-a.e. defined and $\nu$-a.e. defined and, respectively, in $L^{1}(\mu)$ and $L^{1}(\nu)$, satisfying: (c.iii) the formula [*], as above.

Acquainted to the product measure, using proposition 2.1.38, we can define the Lesbegue measure $m^{n}$ on $\mathbb{R}^{n}, n \in \mathbb{N}$, as $\overline{m \times \ldots \times m}$ ( $n$ times), being each $m$ the Lesbegue measure on $\left(\mathbb{R}, \mathcal{B}_{\mathbb{R}}\right)$ (or $(\mathbb{R}, \mathcal{L})$, it is equivalent). It's domain will be denoted $\mathcal{L}^{n}$, with $\mathcal{B}_{\mathbb{R}^{n}} \subset \mathcal{L}^{n}$. Again, by an abuse of notation, we may refer to $m^{n}$ as both the standard Lesbegue measure on $\mathcal{L}^{n}$ or it's restriction to $\mathcal{B}_{\mathbb{R}^{n}}$. More, if the context is clear, we may refer to $m^{n}$ simply by $m$.

The following proposition about regularity is a direct consequence of proposition 2.1.14 applied to $m$.

Proposition 2.1.40 (Regularity of Lesbegue measure on $\mathbb{R}^{n}$ ). For all $E \in \mathcal{L}^{n}$ :

(i) $m^{n}(E)=\inf \left\{m^{n}(U): U\right.$ open, $\left.E \subset U \subset \mathbb{R}^{n}\right\}$ (outer regularity)

(ii) $m^{n}(E)=\sup \left\{m^{n}(K): K\right.$ compact, $\left.K \subset E \subset \mathbb{R}^{n}\right\}$ (inner regularity)

(iii) $E=V \backslash N_{1}$, where $V \in G_{\delta}\left(\mathbb{R}^{n}\right)$ (the set of countable intersections of open sets of $\mathbb{R}^{n}$ ) and $m^{n}\left(N_{1}\right)=0$.

(iv) $E=H \cup N_{2}$, where $H \in F_{\sigma}\left(\mathbb{R}^{n}\right)$ (the set of countable unions of closed sets of $\mathbb{R}^{n}$ ) and $m^{n}\left(N_{2}\right)=0$.

(v) such that $m^{n}(E)<\infty$, it holds that $\forall \epsilon>0, \exists\left(R_{j}\right)_{j=0}^{n}$ a finite collection of disjoint rectangles whose sides are intervals such that $\mu\left(E \triangle \dot{\cup}_{j=0}^{n} R j\right)<\epsilon$. 
The $n$-dimensional Lesbegue measure also inherits a good behavior with respect to translation, and linear transformations (hence dilations and rotations). We will not state these results explicitly.

During the study of dynamical systems, we will be interested in considering, not only finite product spaces, but also countable product spaces. The discussion of this topic is mostly influenced by the appendix of [VO16]. Here, we throw in a little bit of topology, as we did before when discussing finite product of measure spaces. In this discussion, it is very convenient to restrict ourselves to probabilities spaces. We adopt this restriction.

Let $\left(X_{j}, \mathcal{A}_{j}, \mu_{j}\right)_{j \in \mathbb{N}}$ be a countable family of probability spaces and $\left(\prod_{j \in \mathbb{N}} X_{j}, \otimes_{j \in \mathbb{N}} \mathcal{A}_{j}\right)$ be the associated product measurable space, defined after proposition 2.1.34.

Once we are dealing with a countable product, the product $\sigma$-algebra is generated by the family $\mathcal{R}$ of rectangles whose $j$-components are in $\mathcal{A}_{j}$ (see discussion after proposition 2.1.34).

These rectangles are, in turn, obtained as countable intersections of elements in the family $\mathcal{C}$ of (measurable) cylinders $\left[m ; B_{m}, \ldots, B_{m+k}\right]=\left\{x=\left(x_{0}, x_{1}, \ldots\right) \in \prod_{j \in \mathbb{N}} X_{j}: x_{m} \in B_{m}, \ldots, x_{m+k} \epsilon\right.$ $\left.B_{m+k}\right\}, m \geq 0, k \geq 0, B_{j} \in \mathcal{A}_{j}$ for $m \leq j \leq m+k$. Therefore, the family of (measurable) cylinders $\mathcal{C}$ also generates the product $\sigma$-algebra, i.e., $\sigma(\mathcal{C})=\bigotimes_{j \in \mathbb{N}} \mathcal{A}_{j}$.

Using the same notation and attitude of propositions 2.1.11 and 2.1.36, the family $\mathcal{C}$ is also an elementary family. So $\mathcal{A}(\mathcal{C})=\mathcal{D}(\mathcal{C})$ and $\sigma(\mathcal{D}(\mathcal{C}))=\sigma(\mathcal{C})=\bigotimes_{j \in \mathbb{N}} \mathcal{A}_{j}$.

If each $X_{j}$ is equipped with a topology $\tau_{j}$ (in which case we assume $\mathcal{A}_{j}=\mathcal{B}_{X_{j}}$ ), the product space $\prod_{j \in \mathbb{N}} X_{j}$ is equipped with the product topology $\otimes_{j \in \mathbb{N}} \tau_{j}$, the coarest topology on the product space making all the projections continuous (as we know, $\mathcal{B}_{\prod_{j \in \mathbb{N}} X_{j}}$ coincides with $\otimes_{j \in \mathbb{N}} \mathcal{B}_{X_{j}}$ whenever each $\tau_{j}$ has a countable basis, see proposition 2.1.35). Additionally, if each $\left(X_{j}, \tau_{j}\right)$ is compact, so will be $\left(\prod_{j \in \mathbb{N}} X_{j}, \prod_{j \in \mathbb{N}} \tau_{j}\right)$, by the Tychonoff's theorem from topology.

Also, it is a well known result in topology that, when the product is more than finite, the product topology will not generally coincide with the box topology (that one having as basis rectangles whose $j$-components are in $\tau_{j}$ ). Moreover, a countable product of open sets $\prod_{j=0}^{\infty} U_{j}, U_{j} \in \tau_{j}$, is in the product topology if, and only if, $U_{j}=X_{j}$ for all but a finitely many $j$ 's. These sets are called (topological) cylinders and denote them pretty much as before: $\left[m ; B_{m}, \ldots, B_{m+k}\right]$, where, now, $B_{j} \in \tau_{j}$. They form a basis for the product topology. Accordingly, here, a countable intersection of them, consists of a rectangle, but generally not of an open set (as countable intersections of open sets are not necessarily open).

Now, is easy to assign prototypical measures to the family of cylinders. These assignments will be extended, using the Caratheodory's construction, to the $\sigma$-algebra generated by them, i.e., to the product $\sigma$-algebra, defining the product measure $\bigotimes_{j \in \mathbb{N}} \mu_{j}$. We will not go explicitly into details of this constructions, once the major ideas were already provided.

Proposition 2.1.41 (Kolmogorov extension theorem). Let $\left(X_{j}, \mathcal{A}_{j}, \mu_{j}\right)_{j \in \mathbb{N}}$ be a countable family of probability spaces and $\left(\prod_{j \in \mathbb{N}} X_{j}, \otimes_{j \in \mathbb{N}} \mathcal{A}_{j}\right)$ be the associated product measurable space. Then there exists a unique probability $\otimes_{j \in \mathbb{N}} \mu_{j}$ on $\left(\prod_{j \in \mathbb{N}} X_{j}, \bigotimes_{j \in \mathbb{N}} \mathcal{A}_{j}\right)$ such that $\bigotimes_{j \in \mathbb{N}} \mu_{j}\left(\left[m ; B_{m}, \ldots, B_{m+k}\right]\right)=$ $\mu_{m}\left(B_{m}\right) \cdot \ldots \cdot \mu_{m+k}\left(B_{m+k}\right)$, for all (measurable) cylinder $\left[m ; B_{m}, \ldots, B_{m+k}\right]$.

In particular, a given rectangle $\prod_{n \in \mathbb{N}} A_{n}, A_{n} \in \mathcal{A}_{n}$, is such that $\prod_{j \in \mathbb{N}} A_{n}=\cap_{n \in \mathbb{N}} C_{n}$, where $C_{n}=$ $\left[0 ; A_{0}, \ldots A_{n}\right]$, hence, by continuity from below, $\bigotimes_{j \in \mathbb{N}} \mu_{j}\left(\prod_{j \in \mathbb{N}} A_{n}\right)=\lim _{n} \bigotimes_{j \in \mathbb{N}} \mu_{j}\left(C_{n}\right)$.

The probability space $\left(\prod_{j \in \mathbb{N}} X_{j}, \otimes_{j \in \mathbb{N}} \mathcal{A}_{j}, \bigotimes_{j \in \mathbb{N}} \mu_{j}\right)$ is called the product space of $\left(\left(X_{j}, \mathcal{A}_{j}, \mu_{j}\right)\right)_{j \in \mathbb{N}}$. When $\left(X_{j}, \mathcal{A}_{j}, \mu_{j}\right)$ 's are all equal to a same probability space $(X, \mathcal{A}, \mu)$, the product space $\left(\prod_{j \in \mathbb{N}} X, \otimes_{j \in \mathbb{N}} \mathcal{A}, \bigotimes_{j \in \mathbb{N}} \mu\right)$ can be denoted $\left(X^{\mathbb{N}}, \mathcal{A}^{\mathbb{N}}, \mu^{\mathbb{N}}\right)$, case in which $\mu^{\mathbb{N}}$ is called a Bernoulli measure derived from $\mu$.

In the above case, suppose $X$ is finite, with $n$ elements, $X=\left\{s_{0}, \ldots, s_{n-1}\right\}$, and equipped with the discrete $\sigma$-algebra and the (compact) discrete topology $\mathcal{A}=2^{X}, \tau=2^{X}$. As such, the measure $\mu$ is completely described by knowing $\mu\left(\left\{s_{0}\right\}\right)=p_{0}, \ldots, \mu\left(\left\{s_{n-1}\right\}\right)=p_{n-1}$.

In this situation, every (measurable or topological) cylinder can be written as a finite union of the so called elementary cylinders: $\left[m ; b_{m}, \ldots, b_{m+k}\right]=\left\{x=\left(x_{0}, x_{1}, \ldots\right) \in \prod_{j \in \mathbb{N}} X: x_{m}=b_{m}, \ldots, x_{m+k}=\right.$ $\left.b_{m+k}\right\}, m \geq 0, k \geq 0, b_{j} \in X$ for $m \leq j \leq m+k$. As a consequence, the family $\mathcal{E}$ of elementary cylinders also generates the product $\sigma$-algebra and serve as a basis for the product topology. Case in 
which proposition 2.1.41 could extend only a prototypical measure assigned to elementary cylinders: $\bigotimes_{j \in \mathbb{N}} \mu_{j}\left(\left[m ; b_{m}, \ldots, b_{m+k}\right]\right)=p_{m} \cdot \ldots \cdot p_{m+k}$, for all elementary cylinders $\left[m ; b_{m}, \ldots, b_{m+k}\right]$.

To conclude this topic, also when $X$ is finite, the product topology is not only compact but also metrizable, for example, by the distance $d\left(\left(x_{n}\right)_{n \geq 0},\left(y_{n}\right)_{n \geq 0}\right)=\theta^{N}$, where $\theta \in(0,1)$ is a fixed number and $N=N\left(\left(x_{n}\right)_{n \geq 0},\left(y_{n}\right)_{n \geq 0}\right)$ is the largest $N \geq 0$ such that $x_{i}=y_{i}, \forall|i| \leq N$. When $X=\left\{s_{0}, s_{1}\right\}$, or, easier, $X=\{0,1\}$, an equivalent distance for metrizing the product topology is $\sum_{j \in \mathbb{N}} \frac{\left|x_{j}-y_{j}\right|}{2^{j}}$.

This machinery for countable products can be developed analogously, with minor modifications, for $\mathbb{Z}$ instead of $\mathbb{N}$.

Here we conclude the first part of this review. In the second part, we generalize the concept of measure, by broadening its codomain from $[0, \infty]$ to $\overline{\mathbb{R}}$ or $\mathbb{C}$, then we introduce the concept of Radon-Nikodym derivative and finally present Riesz-Markov representation theorem.

Definition 2.1.42 (Signed measure). Let $(X, \mathcal{A})$ be a measurable space. A signed measure $\nu$ on $(X, \mathcal{A})$ (or $X$, or $\mathcal{A}$, if clear from the context) is a function $\nu: \mathcal{A} \rightarrow \overline{\mathbb{R}}$ such that: (i) $\nu(\varnothing)=0$, (ii) the image of $\nu$ do not contain $-\infty$ and $\infty$ at the same time (might contain one), (iii) if $\left(A_{n}\right)_{n \geq 0}$ is a sequence of disjoint sets in $\mathcal{A}$, then $\nu\left(\dot{\cup}_{n \geq 0} A_{n}\right)=\sum_{n \geq 0} \nu\left(A_{n}\right)^{+}-\sum_{n \geq 0} \nu\left(A_{n}\right)^{-}$(one of the summands must be finite), where $\nu(A)^{+}=\max \{\nu(A), 0\}, \nu(A)^{-}=\max \{-\nu(A), 0\}$.

From now on we will call measures by positive measures.

First is instructive to present natural examples of signed measures that later will be known to characterize them in all generality: if $\mu_{1}$ and $\mu_{2}$ are positive measures on $(X, \mathcal{A})$, where one of them is finite, then $\nu=\mu_{1}-\mu_{2}$ is a signed measure; if $\mu$ is a positive measure on $(X, \mathcal{A})$ and $f: X \rightarrow \overline{\mathbb{R}}$ is a $\mu$-quasi-integrable function, then the set function $\nu: \mathcal{A} \rightarrow \overline{\mathbb{R}}$ defined by $\nu(A)=\int_{A} f d \mu$ is a signed measure.

The following basic properties of signed measures can be compared to those of positive measures.

Proposition 2.1.43 (Properties of signed measures). Let $(X, \mathcal{A}, \nu)$ be a signed measure space. Then:

(i) (No more monotonicity) Try taking a measurable set with negative (signed) measure, and note that $\varnothing$ is a subset with greater measure.

(ii) (No more subadditivity) Try taking two measurable sets with negative measures and whose intersection has also negative measure, and note that the sum of their measures is smaller than the measure of the union.

(iii) (Continuity from below) $\left(A_{n}\right)_{n \geq 0}$ an increasing sequence in $\mathcal{A} \Rightarrow \nu\left(\cup_{n} A_{n}\right)=\lim _{n} \nu\left(A_{n}\right)$.

(iv) (Continuity from above) $\left(A_{n}\right)_{n \geq 0}$ a decreasing sequence in $\mathcal{A}$ such that $\nu\left(A_{j}\right) \in \mathbb{R}$ for some $j \geq 0 \Rightarrow \nu\left(\cap_{n} A_{n}\right)=\lim _{n} \nu\left(A_{n}\right)$.

The example where a signed measure is of the form $\nu(A)=\int_{A} f d \mu$ let us motivate some definitions: when $f$ takes only one sign, say positive, over $A, \nu$ must assign positive values to every measurable subset of $A$, but if $f$ assume different signs over $A$, we may find measurable subsets where $f$ is negative, to which $\nu$ assigns negative values.

Hence, with $\nu$ a signed measure on $(X, \mathcal{A})$, we define $A \in \mathcal{A}$ to be positive (negative, null) if $\nu\left(A^{\prime}\right) \geq 0(\leq 0,=0), \forall A^{\prime} \subset A, A^{\prime} \in \mathcal{A}$ (in the previous motivation, that happens exactly when $f \geq 0(\leq 0,=0) \mu$-a.e. in $A)$. Naturally, a subset of a positive (negative, null) set is also positive (negative, null), as well as a countable union of positive (negative, null) sets.

The following two theorems characterize a signed measure $\nu$ as the difference of two positive measures.

Theorem 2.1.44 (Hahn decomposition). Let $\nu$ be a signed measure on $(X, \mathcal{A})$. Then there exists positive set $P$ and a negative one $N$ such that $P \cup N=X$ and $P \cap N=\varnothing$. This (Hahn) decomposition is essentially unique, since any other pair $P^{\prime}, N^{\prime}$ with the same property has symmetric differences $P \triangle P^{\prime}=N \triangle N^{\prime}$ with $\nu$ measure 0 , i.e., null sets can be allocated freely between what is to be taken as positive and negative parts of the decomposition. 
The full proof of the theorem is intricate, so we give not a sketch but a starting hint that illuminates what goes on. Suppose, with no loss of generality, $\nu$ does not assume the value $\infty$ and define $m$ as the sup of $\nu(A)$ as $A$ ranges over the positive sets (it will always exits, since $\varnothing$ is one such). That gives is a sequence $\left(P_{n}\right)_{n \geq 0}$ of positive sets growing in terms of measure, i.e., $\nu\left(P_{n}\right) \nearrow m$. Define $P=\cup_{n} P_{n}$. It is positive since it is the union of positive sets, and $\nu(P)=m$, by continuity from below. Define $N=X \backslash P$. What is left, the intricate part, is proving that $N$ is negative.

Now we define a concept that will be useful in the following theorem and later on. After the following theorem we define another related concept.

Two signed measures $\nu_{1}$ and $\nu_{2}$ on $(X, \mathcal{A})$ are mutually singular, denoted $\nu_{1} \perp \nu_{2}$, if there exists $A_{1}, A_{2} \in \mathcal{A}$ such that $A_{1} \cup A_{2}=X, A_{1} \cap A_{2}=\varnothing, \nu_{1}\left(A_{2}\right)=0, \nu_{2}\left(A_{1}\right)=0$. Intuitively, this says that $\nu_{1}$ and $\nu_{2}$ assign mass in disjoint regions of the space.

The previous theorem divided the space $X$ in two parts on which the measure $\nu$ is "sign compatible". In the next theorem we want to plug, in each part, positive measures $\mu_{1}$ and $\mu_{2}$ whose difference equals $\nu$, and it is reasonable to expect that $\mu_{1}$ and $\mu_{2}$ are mutually singular.

Theorem 2.1.45 (Jordan decomposition). Let $\nu$ be a signed measure on $(X, \mathcal{A})$. Then there exists unique positive measures $\nu^{+}$and $\nu^{-}$on $(X, \mathcal{A})$ such that $\nu=\nu^{+}-\nu^{-}$and $\nu^{+} \perp \nu^{-}$.

As a sketch proof, note that the candidates for $\nu^{+}$, and $\nu^{-}$, are natural. That is, use Hahn decomposition for $\nu$ to find $P$ and $N$, define $\nu^{+}(A)=\nu(A \cap P)$, and $\nu^{-}(A)=-\nu(A \cap N)$. As defined, $\nu^{+}$and $\nu^{-}$are positive measures such that $\nu=\nu^{+}-\nu^{-}$. Uniqueness follow from the essential uniqueness of Hahn decomposition.

With the Jordan decomposition of $\nu=\nu^{+}-\nu^{-}$, we can define a positive measure called the total variation of $\nu,|\nu|=\nu^{+}+\nu^{-}$.

A definition that will come up later side by side with mutual singularity is absolute continuity. A signed measure $\nu$ on $(X, \mathcal{A})$ is absolutely continuous with respect to a positive measure $\mu$ on $(X, \mathcal{A})$, denoted $\nu \ll \mu$, if $\mu(E)=0 \Rightarrow \nu(E)=0$. Clearly $\nu \ll \mu \Leftrightarrow|\nu| \ll \mu \Leftrightarrow \nu^{+} \ll \mu$ and $\nu^{-} \ll \mu$.

We should interpret mutual singularity and absolute continuity as antithetical, in the sense that if $\nu \perp \mu$ and $\nu \ll \mu$, then $\nu \equiv 0$.

The following proposition characterizes absolute continuity under finiteness and explains why we call it like that.

Proposition 2.1.46 (Characterization of absolute continuity under finiteness). Let $\nu$ be a finite signed measure and $\mu$ a positive measure on $(X, \mathcal{A})$. Then $\nu \ll \mu \Leftrightarrow \forall \epsilon>0, \exists \delta>0$ such that $|\nu|(A)<\epsilon$ whenever $\mu(A)<\delta$.

A direct and important use of the concept of total variation, introduced above, is to write $\nu$ as $f d \mu$ for some positive measure $\mu$ and $\mu$-quasi-integrable $f$. For such, use Hahn's decomposition $X=P \cup N$ and Jordan's decomposition $\nu=\nu^{+}-\nu^{-}$, and let be $\mu=|\nu|$ and $f=\mathcal{X}_{P}-\mathcal{X}_{N}$. Note $f d \mu(A)=\int_{A} \mathcal{X}_{P}-\mathcal{X}_{N} d\left(\nu^{+}+\nu^{-}\right)=\int_{A} \mathcal{X}_{P} d \nu^{+}-\int_{A} \mathcal{X}_{N} d \nu^{+}+\int_{A} \mathcal{X}_{P} d \nu^{-}-\int_{A} \mathcal{X}_{N} d \nu^{-}=\nu^{+}(A \cap P)-$ $\nu^{+}(A \cap N)+\nu^{-}(A \cap P)-\nu^{-}(A \cap N)=\nu^{+}(A)-\nu^{-}(A)=\nu(A)$. Therefore $\nu=f d \mu$.

We can easily define integrals with respect to signed measures. Let $\nu$ be a signed measure on $(X, \mathcal{A})$ and $f$ be a measurable function on $(X, \mathcal{A})$ (taking values in $K=\mathbb{C},[0, \infty]$ or $\overline{\mathbb{R}}$ ), such that $f \in L^{1}\left(\nu^{+}\right)$or $f \in L^{1}\left(\nu^{-}\right)$. Now define $\int f d \nu:=\int f d \nu^{+}-\int f d \nu^{-} \in K$. Here $L^{1}(\nu, K)$ is the set of $K$-valued functions whose integral is defined and finite. It is no surprise that $L^{1}(\nu, K)=$ $L^{1}\left(\nu^{+}, K\right) \cap L^{1}\left(\nu^{-}, K\right)$ (when $K=\mathbb{C}$ this is less important, since every function to which an integral $\int \cdot d \eta$ is assigned is in $L^{1}(\eta, \mathbb{C})$ ). Note that we can still assign (infinite) integrals to some functions outside $L^{1}(\nu, K)$ (when considering $K=[0, \infty]$ or $\overline{\mathbb{R}}$ ).

Proposition 2.1.47 (Properties of the total variation of signed measures). Let $\nu, \nu_{1}, \nu_{2}$ be signed measures on $(X, \mathcal{A})$. Then:

(i) $|\nu(A)| \leq|\nu|(A), \forall A \in \mathcal{A}$.

(ii) $L^{1}(\nu, \overline{\mathbb{R}})=L^{1}(|\nu|, \overline{\mathbb{R}})$ and $f \in L^{1}(\nu, \overline{\mathbb{R}}) \Rightarrow\left|\int f d \nu\right| \leq \int|f| d|\nu|$.

(iii) $|\nu|(A)=\sup \left\{\left|\int_{A} f d \nu\right|:|f| \leq 1\right\}$.

(iv) if $\nu_{1}$ and $\nu_{2}$ both omit an infinity of the same sign, $\left|\nu_{1}+\nu_{2}\right| \leq\left|\nu_{1}\right|+\left|\nu_{2}\right|$. 
Now we introduce complex measures.

Definition 2.1.48 (Complex measure). Let $(X, \mathcal{A})$ be a measurable space. A complex measure $\nu$ on $(X, \mathcal{A})$ (or $X$, or $\mathcal{A}$, if clear from the context) is a function $\nu: \mathcal{A} \rightarrow \mathbb{C}$, whose real and imaginary parts are, respectively, $\nu_{r}$ and $\nu_{i}$, such that: (i) $\nu(\varnothing)=0$, (ii) if $\left(A_{n}\right)_{n \geq 0}$ is a sequence of disjoint sets in $\mathcal{A}$, then $\nu\left(\dot{\cup}_{n \geq 0} A_{n}\right)=\nu_{r}\left(\dot{\cup}_{n \geq 0} A_{n}\right)+i \nu_{i}\left(\dot{\cup}_{n \geq 0} A_{n}\right)=\left(\sum_{n \geq 0} \nu_{r}\left(A_{n}\right)^{+}-\sum_{n \geq 0} \nu_{r}\left(A_{n}\right)^{-}\right)+$ $i\left(\sum_{n \geq 0} \nu_{i}\left(A_{n}\right)^{+}-\sum_{n \geq 0} \nu_{i}\left(A_{n}\right)^{-}\right)$(all of the summands must be finite), where $\nu_{j}(A)^{+}=\max \left\{\nu_{j}(A), 0\right\}$, $\nu_{j}(A)^{-}=\max \left\{-\nu_{j}(A), 0\right\}, j \in\{r, i\}$.

Note that the real and imaginary parts of $\nu, \nu_{r}$ and $\nu_{i}$, are signed measures. Natural example in this case is to take two signed measures $\mu_{1}$ and $\mu_{2}$ on $(X, \mathcal{A})$ and define $\nu=\mu_{1}+i \mu_{2}$ or take a positive measure $\mu$ on $(X, \mathcal{A})$ and a $\mu$-quasi-integrable function $f: X \rightarrow \mathbb{C}$, and define the set function $\nu: \mathcal{A} \rightarrow \mathbb{C}$ given by $\nu(A)=\int_{A} f d \mu$. In both this cases, $\nu$ is a complex measure.

Here continuity from above and below still holds, but no analogous to Hahn or Jordan decompositions in the sense that a complex measure $\nu$ will be separated in mutually singular real and imaginary parts instead of positive and negative.

Two complex measures $\nu$ and $\mu$ on $(X, \mathcal{A})$ are mutually singular if $\mu_{a} \perp \nu_{b}, \forall a, b \in\{r, i\}$.

A complex measure $\nu$ on $(X, \mathcal{A})$ is absolutely continuous with respect to a positive measure $\mu$ on $(X, \mathcal{A})$, denoted $\nu \ll \mu$ if $\nu_{r} \ll \mu$ and $\nu_{i} \ll \mu$.

Also, we define integrals with respect to complex measures. Let $\nu$ be a complex measure on $(X, \mathcal{A})$ and $f$ be a measurable function on $(X, \mathcal{A})$ (taking values in $K=\mathbb{C},[0, \infty]$ or $\overline{\mathbb{R}}$ ), such that $f \in L^{1}\left(\nu_{r}\right)$ and $f \in L^{1}\left(\nu_{i}\right)$. Now define $\int f d \nu:=\int f d \nu_{r}+i \int f d \nu_{i} \in \mathbb{C}$. Here $L^{1}(\nu, K)$ is the set of $K$-valued functions whose integral is defined and finite. It is no surprise that $L^{1}(\nu, K)=$ $L^{1}\left(\nu_{r}, K\right) \cap L^{1}\left(\nu_{i}, K\right)$. Note we only assign integrals to functions in $L^{1}(\nu, K)$.

We did not define the total variation of a complex measure. This definition will make sense after the next theorem. First let's motivate it.

Now signed (complex) measures of the form $\nu(A)=\int_{A} f d \mu$, where $\mu$ is a positive measure and $f$ a $\overline{\mathbb{R}}$-valued ( $\mathbb{C}$-valued) $\mu$-quasi-integrable function, will play a major role. We may write them as $\nu=f \mu$ and $d \nu=f d \mu$, in the sense that $\int \mathcal{X}_{A} d \nu=\nu(A)=\int_{A} f d \mu=\int \mathcal{X}_{A} f d \mu$. Note that $f \mu \ll \mu$. Is every signed (complex) measure $\nu$ absolutely continuous with respect to a positive measure $\mu$ of the form $f \mu$ for some $\overline{\mathbb{R}}$-valued ( $\mathbb{C}$-valued) $\mu$-quasi-integrable function $f$ ? (I)

One explicit example asks us to go back to Lesbegue-Stieltjes measures. In that context, from a non-decreasing right-continuous $F: \mathbb{R} \rightarrow \mathbb{R}$ we could induce a measure $\mu_{F}$ on $\left(\mathbb{R}, \mathcal{B}_{\mathbb{R}}\right)$ such that $\mu_{F}((a, b])=F(b)-F(a)$. Suppose we start with one such $F$ with the additional property that $F \in C^{1}$. By the fundamental theorem of calculus, $\mu_{F}((a, b])=F(b)-F(a)=\int_{a}^{b} F^{\prime}(x) d x=$ $\int_{(a, b]} F^{\prime} d m=F^{\prime} m((a, b])$ (Riemann integral and Lesbegue integral coincide whenever the first is defined - this discussion was omitted). Then $\mu_{F}=F^{\prime} m$. In this case, is every positive measure $\nu$ absolutely continuous with respect to $m$ of the form $f m$ for some $[0, \infty]$-valued $m$-quasi-integrable function $f$ ?

These last two (reciprocal) questions will admit a positive answer, due to the next theorem, when assuming $\nu$ and $\mu$ are $\sigma$-additive.

Even more, given a signed (complex) measure $\nu$ and a positive measure $\mu$, we want to come up with some way of writing $\nu$ as the sum of (i) a signed (complex) measure to be understood as a "deformation" or "transformation" of $\mu$, i.e., $f \mu$ for some $\overline{\mathbb{R}}$-valued ( $\mathbb{C}$-valued) $\mu$-quasi-integrable function $f$, and (ii) a signed (complex) measure $\lambda$ mutually singular to $\mu$ (hence to $f \mu$ ). In some really vague sense, we are writing $\nu$ as a "factor" $f$ times $\mu$ plus $\lambda$, where $\lambda$ is dissociated from $\mu$. This is one reason why $f$ is to be understood as derivative, and we denote it by $f=d \nu / d \mu$, which is consistent with the notation $d \nu=f d \mu$. Also, the last example also contributes to this understanding, since $d \mu_{F}=F^{\prime} d m \Rightarrow d \mu_{F} / d m=F^{\prime}$.

When we consider $\mu=m$, the Lesbegue measure on $\left(\mathbb{R}^{n}, \mathcal{B}_{\mathbb{R}^{n}}\right), d \nu / d \mu$ is a function mapping $x$ to the limit of a quotient of $\nu$ over $\mu$ calculated on adequate measurable sets containing (or almost that) $x$ and shrinking adequately towards $x$. This is an explicit derivative-like concept. We will state this result after presenting Lesbegue-Radon-Nikodym theorem and related properties. 
The theorem answers our motivating questions in two parts.

Theorem 2.1.49 (Lesbegue-Radon-Nikodym, signed version). Let $\nu$ be a $\sigma$-finite signed measure and $\mu$ be a $\sigma$-finite positive measure, both on $(X, \mathcal{A})$. Then:

(i) (Lesbegue decomposition) There exists unique signed measures $\nu_{a c}$ and $\nu_{m s}$ on $(X, \mathcal{A})$ such that $\nu_{a c} \ll \mu, \nu_{m s} \perp \mu$ and $\nu=\nu_{a c}+\nu_{m s}$, which are themselves $\sigma$-finite.

(ii) (Radon-Nikodym derivative) If $\nu \ll \mu$, there exists a $\mu$-quasi-integrable function $f: X \rightarrow \overline{\mathbb{R}}$ such that $\nu=f \mu$. This $f$ is essentially unique in the sense that another $f^{\prime}$ with the same property is equal to $f \mu$-a.e. The class of equivalence $[f]$ (or one of its representatives, as the context will make clear) is denoted $d \nu / d \mu$, called the Radon-Nikodym derivative of $\nu$ with respect to $\mu$.

(iii) In (i), using (ii) for $\nu_{a c}$, one can write $\nu=f \mu+\nu_{m s}$.

The proof is intricate. What follows is a starting hint that clarifies things. Suppose $\nu$ and $\mu$ are positive and finite. As a motivation for the argument, imagine we know beforehand a positive measure $\nu_{m s}$ and a $[0, \infty]$-valued measurable function $f$ such that $\nu=\nu_{m s}+f \mu$ and $\nu_{m s} \perp \mu$ (let $M$ be the set where $\nu_{m s}$ is null and $S$ be that were $\mu$ is null). In this case, $f \mu$ would be less than or equal to $\nu$ (since $\nu(A)=\nu_{m s}+\int_{A} f d \mu \geq \int_{A} f d \mu, \forall A \in \mathcal{A}$ ) and whenever a $[0, \infty]$-valued measurable function $g$ is such that $g \mu$ is less than or equal to $\nu$, it will have to be less than or equal to $f \mu$ as well $\left(\int_{A} g d \mu=\int_{A \cap M} g d \mu \leq \nu(A \cap M)=\int_{A \cap M} f d \mu=\int_{A} f d \mu, \forall A \in \mathcal{A}\right)$. In other words, $\forall A \in \mathcal{A}$, $f$ is basically maximal in the set of $([0, \infty]$-valued measurable) functions that integrated with respect to $\mu$ in $A$ are less than or equal to $\nu$.

Now define $\mathcal{F}=\left\{f: X \rightarrow[0, \infty]\right.$ measurable $\left.: \int_{A} f d \mu \leq \nu(A), \forall A \in \mathcal{A}\right\}, 0 \in \mathcal{F}$. Let be $m=$ $\sup \left\{\int_{X} f d \mu: f \in \mathcal{F}\right\} \leq \nu(X)<\infty$. Actually, this sup is reached: let $\left(g_{n}\right)_{n \geq 0}$ be a sequence in $\mathcal{F}$ whose integral converges to $m$ and note that $g, h \in \mathcal{F} \Rightarrow \max \{g, h\} \in \mathcal{F}$, then take the sequence whose entries are $f_{n}=\max \left\{g_{0}, \ldots, g_{n}\right\} \in \mathcal{F}$ and $f=\lim f_{n}$. The integral of $f_{n}$ will be squeezed by those of $g_{n}$ 's, hence it also converges (monotonically) to $m$. By the monotone convergence theorem, applied using any $A \in \mathcal{A}$, it follows that $f \in \mathcal{F}$ and $\int f d \mu=m$ (considering $A=X$ ).

This $f$ is our candidate maximal function (we chose it to be maximal with $A=X$, but aftermath it will be like that for any $A \in \mathcal{A}$ ). Now define $\nu_{m s}:=\nu-f \mu$. For the general result, one would still have to prove $\nu_{m s} \perp \mu$, take care of uniqueness, generalize for $\mu$ and $\nu \sigma$-finite, signed $\nu$, etc. The generalization for complex measures, stated next, leverages on the result for signed measures.

Theorem 2.1.50 (Lesbegue-Radon-Nikodym, complex version). Let $\nu$ be a complex measure and $\mu$ be a $\sigma$-finite positive measure, both on $(X, \mathcal{A})$. Then:

(i) (Lesbegue decomposition) There exists unique complex measures $\nu_{a c}$ and $\nu_{m s}$ on $(X, \mathcal{A})$ such that $\nu_{a c} \ll \mu, \nu_{m s} \perp \mu$ and $\nu=\nu_{a c}+\nu_{m s}$.

(ii) (Radon-Nikodym derivative) If $\nu \ll \mu$, there exists a $\mu$-integrable function $f: X \rightarrow \mathbb{C}$ such that $\nu=f \mu$.

Everything else is identical to the last theorem.

The differential notation has even more reasons.

Proposition 2.1.51 (Properties Radon-Nikodym derivative, signed version). Let $\nu, \nu_{1}, \nu_{2}$ be a $\sigma$ finite signed measure and $\mu, \lambda \sigma$-finite positive measures, all on $(X, \mathcal{A})$, such that $\nu, \nu_{1}, \nu_{2} \ll \mu$ and $\mu \ll \lambda$. Then:

(i) $d\left(\nu_{1}+\nu_{2}\right) / d \mu=d \nu_{1} / d \mu+d \nu_{2} / d \mu$.

(ii) $g \in L^{1}(\nu, \overline{\mathbb{R}}) \Rightarrow g d \nu / d \mu \in L^{1}(\mu, \overline{\mathbb{R}})$ and $\int g d \nu=\int g d \nu / d \mu d \mu$.

(iii) $\nu \ll \lambda$ and $d \nu / d \mu d \mu / d \lambda=d \nu / d \lambda, \lambda$-a.e..

(iv) if it also holds that $\lambda \ll \mu, d \mu / d \lambda d \lambda / d \mu=1, \mu$-a.e. and $\lambda$-a.e..

Now we present, omitting details, the aforementioned quotient interpretation to the derivative. After that we close the derivative subject with an application, defining total variation of complex measures.

The next theorem requires a new definition. Let $(X, \tau)$ be a Hausdorff topological space and $\left(X, \mathcal{B}_{X}, \mu\right)$ be a Borelian measure space (Hausdorff is to guarantee every compact set is Borelian). For $p \in[1, \infty]$, define $L_{l o c}^{p}(\mu, \mathbb{C}):=\left\{f: X \rightarrow \mathbb{C}: f\right.$ is measurable and $f \mathcal{X}_{K} \in L^{p}(\mu, \mathbb{C}), \forall K \subset$ 
$X, K$ compact $\}$. This is set of locally integrable functions. Locally integrable functions are required to be well behaved with respect to integration on compact sets, but are allowed to grow rapidly at "infinity".

Theorem 2.1.52 (Lesbegue differentiation theorem). Let $\left(\mathbb{R}^{n}, \mathcal{B}_{\mathbb{R}^{n}}, \nu\right)$ be a signed or complex Borelian measure space, $\left(\mathbb{R}^{n}, \mathcal{B}_{\mathbb{R}^{n}}, m\right)$ be the Lesbegue measure space and $B_{r}(x)$ be the open ball of radius $r$ and center $x$ on $\mathbb{R}^{n}$. Then:

a) if $f \in L_{\text {loc }}^{1}(m, \mathbb{C})$, then $\lim _{r \rightarrow 0} \frac{\int_{B_{r}(x)} f(y) d \nu(y)}{m\left(B_{r}(x)\right)}=f(x)$, m-a.e. $x \in \mathbb{R}^{n}$.

b) writing $\nu=f m+\lambda$ (using Lesbegue-Radon-Nikodym theorem), then $\lim _{r \rightarrow 0} \frac{\nu\left(B_{r}(x)\right)}{m\left(B_{r}(x)\right)}=f(x)=$ $\frac{d \nu}{d m}(x)$, m-a.e. $x \in \mathbb{R}^{n}$.

A direct consequence of the previous theorem, considering $\nu=m$, is the so called Lesbegue density theorem.

Theorem 2.1.53 (Lesbegue density theorem). Let $\left(\mathbb{R}^{n}, \mathcal{B}_{\mathbb{R}^{n}}, m\right)$ be the Lesbegue measure space and $A \in \mathcal{B}_{\mathbb{R}^{n}}$ such that $m(A)>0$. Then $\lim _{r \rightarrow 0} \frac{m\left(B_{r}(x) \cap A\right)}{m\left(B_{r}(x)\right)}=1$, m-a.e. $x \in A$.

Finally, we conclude this topic with a useful application mentioned a while ago: the total variation of a complex measure $\nu$, a positive measure denoted by $|\nu|$. It is defined first choosing some $\mu$ such that $\nu \ll \mu$ (there will always be some, e.g., $\left.\left|\nu_{r}\right|+\left|\nu_{i}\right|\right)$, then taking $f$ such that $\nu=f \mu$ and letting be $|\nu|=|f| \mu$ (this definition is independent of choice of $\mu$ and associated $f$ ). It coincides with the total variation of finite signed measure based on Jordan decomposition. The following properties will hold.

Proposition 2.1.54 (Properties of the total variation of complex measures). Let $\nu, \nu_{1}, \nu_{2}$ be complex measures on $(X, \mathcal{A})$. Then:

(i) $|\nu(A)| \leq|\nu|(A), \forall A \in \mathcal{A}$.

(ii) $\nu \ll|\nu|$ and $d \nu / d|\nu|=1,|\nu|$.a.e..

(iii) $L^{1}(\nu, \mathbb{C})=L^{1}(|\nu|, \mathbb{C})$ and $f \in L^{1}(\nu, \mathbb{C}) \Rightarrow\left|\int f d \nu\right| \leq \int|f| d|\nu|$.

(iv) $|\nu|(A)=\sup \left\{\left|\int_{A} f d \nu\right|:|f| \leq 1\right\}$.

(v) $\left|\nu_{1}+\nu_{2}\right| \leq\left|\nu_{1}\right|+\left|\nu_{2}\right|$.

We now shift to the last topic of the second part of this section, Riesz-Markov representation theorem.

Until now, $X$ was, at most, assumed to be equipped with a topology $\tau$. Now assume it has more structure and $(X, \tau)$ is a locally compact Hausdorff topological space (denoted shortly, $X$ is LCH). These topological spaces are quite general and encompass all those cases we will be interested in. To remember, (a) locally compact means every point in $X$ has a compact neighbourhood, i.e., $\forall x \in X, \exists L$ compact $\subset X$, such that $\exists U$ open $\subset L$, with $x \in U$; and (b) Hausdorff means every pair of points in $X$ are neighbourhood-separable, i.e., $\forall x, y \in X, \exists U, V \subset X$, open sets, with $x \in U, y \in V$ and $U \cap V=\varnothing$.

Definition 2.1.55 (Radon measures). On a measurable space $\left(X, \mathcal{B}_{X}\right)$, where $X$ is $L C H$, a positive Radon measure $\nu$ is a Borelian measure that:

(i) assigns finite values for compact measurable sets (hence being locally finite). $\mathcal{B}_{X}$

(ii) is outer regular for all measurable sets, i.e., $\eta(B)=\inf \left\{\eta(U): B \subset U, U\right.$ open $\left.\in \mathcal{B}_{X}\right\}, \forall B \in$

(iii) is inner regular for all open sets, i.e., $\eta(U)=\sup \left\{\eta(L): L \subset U, L\right.$ compact $\left.\in \mathcal{B}_{X}\right\}, \forall U$ open $\in \mathcal{B}_{X}$.

A signed Radon measure $\nu$ in the same space is one such that $\nu^{+}$and $\nu^{-}$(from Jordan decomposition) are positive Radon measures.

A complex Radon measure $\nu$ in the same space is one such $\nu_{r}$ and $\nu_{i}$ are signed Radon measures.

Radon measures are distinguished because they behave specially well with respect to the topology in $X$, i.e., it assigns finite values to compact sets and exhibit nice regularity/approximation properties using topologically meaningful sets. The next proposition summarizes some further regularity results. 
Proposition 2.1.56 (Regularity properties of Radon measures). Let $\left(X, \mathcal{B}_{X}, \nu\right)$ be a measure space, where $X$ is $L C H$ and $\nu$ is a Radon measure. Then:

(i) $\nu$ is inner regular on every measurable $\sigma$-finite set $E$ (i.e., one that can be written as a countable union of measurable sets of finite measure)

(ii) if $\nu$ is $\sigma$-finite, $\forall E \in \mathcal{B}_{X}, \epsilon>0, \exists$ an open $U$ and a closed $F$ such that $F \subset E \subset U$ and $\nu(U \backslash F)<\epsilon$, in particular $\nu(E \triangle U)<\epsilon$ and $\nu(E \triangle F)<\epsilon$

(iii) if $\nu$ is $\sigma$-finite, $\forall E \in \mathcal{B}_{X}, \exists A \in F_{\sigma}$ (countable union of closed sets) and $B \in G_{\delta}$ (countable intersection of open sets) such that $A \subset E \subset B$ and $\mu(B \backslash A)=0$.

For $K \in\{\mathbb{C}, \mathbb{R},[0, \infty]\}, X$ LCH, define:

(a) $\mathcal{M}_{r}(X, K)=\left\{\nu: \mathcal{B}_{X} \rightarrow K: \nu\right.$ Radon measure $\}$.

(b) $C_{b}(X, K)=\{f: X \rightarrow K ; f$ is continuous and bounded $\}$, where $f$ is bounded if there exists $c \in K$ such that $|f(x)| \leq c, \forall x \in X$.

(c) $C_{0}(X, K)=\{f: X \rightarrow K$; $f$ is continuous and vanishes at infinity $\}$, where $f$ vanishes at infinity if $\forall \epsilon>0,\{x \in X:|f(x)| \geq \epsilon\}$ is compact.

(d) $C_{c}(X, K)=\{f: X \rightarrow K ; f$ is continuous and $\operatorname{supp}(f)$ is compact $\}$, where $\operatorname{supp}(f)=$ $\{x \in X: f(x) \neq 0\}$ is compact.

Obviously, $C_{c}(X, K) \subset C_{0}(X, K) \subset C_{b}(X, K)$. Please refer to section 2.2 for functional analysis considerations regarding theses sets, the associated uniform norm, $\|f\|_{u}=\sup _{x \in X}|f(x)|$, and the definition of the dual sets of $C_{i}(X, K), C_{i}(X, K)^{*}, i \in\{b, 0, c\}, K \in\{\mathbb{C}, \mathbb{R}\}$. These concepts are going to be used next and should checked in section 2.2 if necessary. Additionally, here we define $C_{i}(X, K)^{+}=\left\{T: C_{i}(X, K) \rightarrow K ; T\right.$ is linear and positive, i.e., $T(f) \geq 0$ if $\left.f \geq 0\right\}$, for $i \in\{b, 0, c\}$ and $K \in\{\mathbb{C}, \mathbb{R},[0, \infty]\}$.

It seems that positivity says nothing about continuity, but the following proposition show us the opposite: positive functionals on $C_{c}(X, K)$, when restricted to the subset of continuous functions of compact support contained in a compact set $L$, are $\left(\|\cdot\|_{u},|\cdot|\right)$-continuous. The proof of this proposition is (short but) mostly technical, so we omit it.

Proposition 2.1.57 (Positive functionals and continuity). Let $T: C_{c}(X, K) \rightarrow K, K \in\{\mathbb{C}, \mathbb{R}\}$ be $a$ positive functional. Then, for any compact $L \subset X, \exists C_{L}$ such that $|T(f)| \leq C_{L}\|f\|_{u}, \forall f \in C_{c}(X, K)$ with $\operatorname{supp}(f) \subset L$.

If $\mu \in \mathcal{M}_{r}(X,[0, \infty])$, the functional $f \in C_{c}(X, K) \mapsto \int f d \mu \in K\left(f \in C_{c}(X, K) \Rightarrow f \in L^{1}(\mu, K)\right)$ is in $C_{c}(X, K)^{+}$, for $K \in\{\mathbb{C}, \mathbb{R}\}$ (for $C_{b}(X, K)^{+}$and $C_{0}(X, K)^{+}$, the associated functionals would not be generally well defined).

The first version of Riesz-Markov representation theorem provides a reciprocal characterization for these positive functionals in $C_{c}(X, K)^{+}, K \in\{\mathbb{C}, \mathbb{R}\}$.

Theorem 2.1.58 (Riesz-Markov representation theorem for $C_{c}(X, K)^{+}$). Let $X$ be LCH and T $\epsilon$ $C_{c}(X, K)^{+}, K \in\{\mathbb{C}, \mathbb{R}\}$. Then there exists a unique $\mu \in \mathcal{M}_{r}(X,[0, \infty])$ such that $T(f)=\int f d \mu, \forall f \in$ $C_{c}(X, K)$. Moreover:

(i) $\mu(U)=\sup \left\{T(f): f \in C_{c}(X, K), 0 \leq f \leq 1, \operatorname{supp}(f) \subset U\right\}$, for all open set $U$.

(ii) $\mu(L)=\inf \left\{T(f): f \in C_{c}(X, K), \mathcal{X}_{L} \leq f\right\}$, for all compact set $L$.

Again, the proof is intricate. What follows is a hint. The uniqueness argument gives us the insight how to proceed. Suppose we have $\mu \in \mathcal{M}_{r}(X,[0, \infty])$ such that $T(f)=\int f d \mu, \forall f \in C_{c}(X, K)$, and $U$ an open set. Then, for all $f \in C_{c}(X, K)$ such that $0 \leq f \leq 1$ and $\operatorname{supp}(f) \subset U$, by monotonicity, $T(f) \leq \mu(U)$. On the other hand, by inner regularity we can find a compact $L \subset U$ such that $\mu(L) \leq \mu(U)$ but whose measure can be as close to that of $U$ as desired. Knowing that we can always take a continuous $f$ with $f=1$ in $L$ and $\operatorname{supp}(f) \subset U$ (Urysohn's lemma, from topology), it follows that (i) holds. So, whatever $\mu$ representing $T$ we start with, its values in open sets are determined by $T$, hence, by outer regularity, its values in all measurable sets will also be determined by $T$. That is uniqueness. For existence, the previous ideia suggests we define $\mu$ as in (i) on open sets, use it as a pre outer measure and leverage on Caratheodory's construction the come up with a candidate measure. This candidate will satisfy all the desired properties. 
We can adapt this version of Riesz-Markov representation theorem to make it say something about the more general space $C_{0}(X, K)^{+}, K \in\{\mathbb{C}, \mathbb{R}\}$. It will say $T \in C_{0}(X, K)^{+}$can be represented with integration against a finite $\mu \in \mathcal{M}_{r}(X,[0, \infty])$. Here is an idea why.

First, let be $\mu \in \mathcal{M}_{r}(X,[0, \infty])$ and $T: C_{c}(X, K) \rightarrow K$, given by $T(f)=\int f d \mu, K \in\{\mathbb{C}, \mathbb{R}\}$. Since it is a linear transformation between normed vector spaces, as stated in section 2.2, $T$ has finite operator norm $\Leftrightarrow T$ is continuous $\Leftrightarrow T$ admits a (unique) continuous extension $\bar{T}$ on $C_{0}(X, K)$. For now, the only implication worth giving a hint is the last $(\Rightarrow)$ : let $f \in C_{0}(X, K)$ and $f_{n} \in C_{c}(X, K)$ be any sequence such that $\left\|f-f_{n}\right\|_{u} \rightarrow 0$ and $\left\|f_{n}\right\|_{u} \nearrow\|f\|_{u}$ (we can find one because $C_{0}(X, K)$ is the completion of $C_{c}(X, K)$ in the $\|\cdot\|_{u}$ norm, as introduced in section 2.2). Since (once $X$ is open) $\mu(X)=\sup \left\{T(f): f \in C_{c}(X, K), 0 \leq f \leq 1\right\} \leq C \cdot 1<\infty$, where $C$ is a bound $T$, we know $\int f d \mu$ is finite and well defined. By the dominated convergence theorem, $T\left(f_{n}\right)=\int f_{n} d \mu \rightarrow \int f d \mu$. Hence, by defining $\bar{T}(f)=\int f d \mu$, we get the desired extension. The reason why this procedure is well defined and other details are discussed in section 2.2. Note we did not prove the equivalence, but only illustrated the extension thing. However, the equivalence is going to be used next.

Given $T \in C_{0}(X, K)^{+}$, its restriction $T^{\prime}$ to $C_{c}(X, K)^{+}$is represented as integration with respect to $\mu \in \mathcal{M}_{r}(X,[0, \infty])$ and admits a continuous extension $T$. So, by the former equivalence, $T^{\prime}$ is a bounded operator, so, $\mu$ is finite (same argument as above) and $T(\cdot)=\int \cdot d \mu$ (by uniqueness of the extension).

The last theorem of this section generalizes the previous result to $C_{0}(X, K)^{*}$, making use of it and the fact that any $T \in C_{0}(X, \mathbb{R})^{*}$ can be written as $T=T^{+}-T^{-}$, with $T^{+}, T^{-}, \in C_{0}(X, \mathbb{R})^{+}$ (this requires proof), thereby making it possible to reduce complex functionals into signed ones, and these into positive. Additionally, please refer to section 2.2 for a discussion of $\mathcal{M}_{r}(X, K)$ as a normed space.

Theorem 2.1.59 (Riesz-Markov representation theorem for $\left.C_{0}(X, K)^{*}\right)$. Let $X$ be LCH and, for $\mu \in \mathcal{M}_{r}(X, K), K \in\{\mathbb{C}, \mathbb{R}\}$, define the functional in $C_{0}(X, K)^{*}, T_{\mu}: C_{0}(X, K) \rightarrow K$, given by

$T_{\mu}(f)=\int f d \mu$. Then the map $\mu \stackrel{\Phi}{\mapsto} T_{\mu}$ from the set of measures $\mathcal{M}_{r}(X, K)$ to the set of functionals $C_{0}(X, K)^{*}$ is a $\left(\|\cdot\|_{m},\|\cdot\|_{\theta}\right)$-isometric (hence continuous and bounded) linear bijection, whose inverse exists and has the same properties, i.e, an isomorphism.

\subsection{Functional analysis and the space of measures}

In this section, only a small fraction of functional analysis will be discussed. The objective is two-fold: (i) to put into light the Riesz-Markov representation theorem and (ii) to use it as a bridge in order to induce topologies in space of measures, discussing specially the weak* topology and its properties. As a supplement, this section omits proofs and arguments of many results presented herein.

It is assumed the reader is used to the concept of a field, denoted by $(K,+, \cdot)$. If the context allows, details can be omitted to say only " $K$ is a field". For example, the sets $\mathbb{R}$ and $\mathbb{C}$ are fields.

It is also assumed the reader is used to the concept of a vector space over a field $K$, of a $K$-vector space, denoted $(V, \oplus, \odot, K)$, with $(K,+, \cdot)$ a field. If the context allows, details can be omitted to say only " $V$ is a $(K-)$ vector space". Also, we will omit the circle around operations in $V$, the symbol . in both cases and any arrows over vectors in $V$.

For example $\mathbb{R}$ is a $\mathbb{R}$-vector space and $\mathbb{C}$ is a $\mathbb{C}$-vector space. More interestingly, for $K \in\{\mathbb{C}, \mathbb{R}\}$, the sets of functions $C_{b}(X, K), C_{0}(X, K), C_{c}(X, K)$, introduced after definition 2.1.55, where $X$ is LHC, equipped with pointwise addition and multiplication are $K$-vector spaces.

First note $\mathbb{R}$ and $\mathbb{C}$ are equipped with their natural modulus function $|$.$| , which satisfy: (i) |a| \geq 0$ and $|a|=0 \Leftrightarrow a=0$, (ii) $|a b|=|a||b|$, (iii) $|a+b| \leq|a|+|b|$.

A normed vector space $(V,\|\cdot\|)$ is a $K$-vector space $V, K \in\{\mathbb{R}, \mathbb{C}\}$, equipped with a function $\|\cdot\|: V \rightarrow \mathbb{R}$ such that (i) $\|v\| \geq 0$ and $\|v\|=0 \Leftrightarrow v=0$, (ii) $\|a v\|=|a|\|v\|$, (iii) $\|v+u\| \leq\|v\|+\|u\|$ (one such function is called a norm). When $(V,\|\cdot\|)$ is complete, i.e., when every Cauchy sequence in $(V,\|\cdot\|)$ is convergent, it is called a Banach space. 
For example, $(\mathbb{R},||$.$) and (\mathbb{C},||$.$) , seen as vector spaces over themselves and equipped with their$ modulus as norms, are Banach spaces.

For $K \in\{\mathbb{C}, \mathbb{R}\}$, one norm that we can define to equip any of the $C_{b}(X, K), C_{0}(X, K), C_{c}(X, K)$ is $\|f\|_{u}=\sup _{x \in X}|f(x)|$. With this norm, $C_{b}(X, K), C_{0}(X, K)$ form a Banach space, while $C_{c}(X, K)$ is a (incomplete) metric space, having $C_{0}(X, K)$ as its completion with respect to the norm $\|\cdot\|_{u}$.

For us, another important example is the set $\mathcal{M}_{r}(X, K), K \in\{\mathbb{C}, \mathbb{R}\}, X \mathrm{LCH}$, introduced after definition 2.1.55, which can be equipped with the norm $\|\nu\|_{m}=|\nu|(X)$, where $|\nu|$ is the total variation (positive) measure of the complex or signed measure $\nu$, all defined in section 2.1. With this norm, this set forms a Banach space.

It is a standard result that a transformation $F$ between arbitrary normed $K$-vector spaces $\left(X,\|\cdot\|_{a}\right)$ and $\left(\mathcal{Y},\|\cdot\|_{b}\right)$ is $\left(\|\cdot\|_{a},\|\cdot\|_{b}\right)$-continuous if and only if is $\left(\|\cdot\|_{a},\|\cdot\|_{b}\right)$-bounded, i.e., there exists $C \in \mathbb{R}$ such that $\|F x\|_{b} \leq C\|x\|_{a}, \forall x \in X$.

Given two normed $K$-vector spaces $\left(X,\|\cdot\|_{a}\right)$ and $\left(\mathcal{Y},\|\cdot\|_{b}\right)$, one can define the set $L(X, \mathcal{Y})=$ $\left\{F: X \rightarrow \mathcal{Y} ; F\right.$ is linear and $\left(\|\cdot\|_{a},\|.\|_{b}\right)$-continuous $\}$. By the previous paragraph, in the definition of $L(X, \mathcal{Y})$ we could have used "bounded" instead of "continuous".

The set $L(X, \mathcal{Y})$ can be equipped with the operator norm $\|F\|_{\theta}=\inf \left\{C \in \mathbb{R}:\|F x\|_{b} \leq C\left\|_{x}\right\|_{a}, \forall x \in\right.$ $x\}=\sup \left\{\|F x\|_{b} /\|x\|_{a}: x \neq \overrightarrow{0}\right\}=\sup \left\{\|F x\|_{b}:\|x\|_{a}=1\right\}$. If $\left(\mathcal{Y},\|\cdot\|_{b}\right)$ is complete, i.e., a Banach space, then $\left(L(X, \mathcal{Y}),\|\cdot\|_{\theta}\right)$ is also going to be.

When the second normed $K$-vector space, $\left(\mathcal{Y},\|\cdot\|_{b}\right)$, is $(K,||$.$) , where K$ is field and subset of $\mathbb{C}$, we denote $L(X, K)=\left\{T: X \rightarrow K ; T\right.$ is linear and $\left(\|\cdot\|_{u},||.\right)$-continuous $\}$ by $X^{*}$, named the dual of $X$. Since $(K,||$.$) is complete, it follows that X^{*}$ is a Banach space. Important examples will be the dual sets of $C_{i}(X, K), i \in\{b, 0, c\}, K \in\{\mathbb{C}, \mathbb{R}\}$.

Also, when dealing with a normed $K$-vector space $\left(\mathcal{X},\|\cdot\|_{a}\right)$ (not necessarily complete) and its completion $\left(\bar{X},\|\cdot\|_{a}\right)$, a Banach space, we have that $X^{\star}$ and $\bar{X}^{\star}$ are isomorphic. That is, given $T \in X^{*}$, let $x \in \bar{X}$, take any $\left(x_{n}\right)_{n \geq 0}$ in $\mathcal{X} \subset \bar{X},\|\cdot\|_{a}$-convergent to $x$, and note that $T\left(x_{n}\right)$ converges in $K$ (since $T$ is bounded, the sequence $T\left(x_{n}\right)$ is also bounded, so there is a convergent subsequence $x_{n_{k}}$, and if there was another one, $x_{n_{k}}^{\prime}$, not converging or converging to another value, $x_{n_{k}}-x_{n_{k}}^{\prime} \in \mathcal{X}$ would $\|\cdot\|_{a}$-converge to $0 \in \mathcal{X}$ while $T$ evaluated at this new sequence would not go to $0 \in K$, a contradiction, because $T$ is continuous), hence by defining $\bar{T}(x)=\lim _{n} T\left(x_{n}\right)$ we obtain $\bar{T} \in \bar{X}^{*}$. The map $T \stackrel{\Theta}{\mapsto} \bar{T}$ is an isometric linear bijection between $X^{*}$ and $\bar{X}^{*}$, whose inverse $\left.\bar{T}_{\stackrel{\Theta^{-1}}{\mapsto}} \bar{T}\right|_{X}$ has the same properties.

Since $C_{0}(X, K)$ is the completion of $C_{c}(X, K)$ under the norm $\|\cdot\|_{u}$, we have that $C_{0}(X, K)^{*}$ and $C_{c}(X, K)^{*}$ are isomorphic, with $\Theta$ presented above serving as an isomorphism.

This is useful since it lets us know there exists an isomorphism of the same kind of that introduced in theorem 2.1.59 between $\mathcal{M}_{r}(X, K)$ and $C_{c}(X, K)^{*}, K \in\{\mathbb{C}, \mathbb{R}\}$, namely $\Gamma=\Theta^{-1} \circ \Phi$, where $\Theta$ was introduced above and $\Phi$ in theorem 2.1.59.

Until now, some new concepts were introduced, enough to enable an enlarged understanding of Riesz-Markov representation theorem (as stated in 2.1.59).

Next, in addition to the topologies already introduced for $C_{i}(X, K), C_{i}(X, K)^{*}$ and $\mathcal{M}_{r}(X, K)$, $K \in\{\mathbb{C}, \mathbb{R}\}, i \in\{b, 0, c\}$, some other ones will be presented. From now until after the next theorem, we are not following any text. From theorem until the end of this section we are following [VO16].

(1) strong topology on $C_{i}(X, K)$ : is the topology $[i] \tau_{s}$ induced by $\|\cdot\|_{u}$.

(i) strong convergence in $C_{i}(X, K): f_{n} \underset{C_{i}}{\stackrel{s}{\rightarrow}} f \Leftrightarrow\left\|f_{n}-f\right\|_{u} \rightarrow 0$.

$\left(1^{*}\right)$ strong topology on $C_{i}(X, K)^{*}$ : is the topology $[i] \tau_{s}^{*}$ induced by $\|\cdot\|_{\theta}$.

(i*) strong convergence in $C_{i}(X, K)^{*}: T_{n} \underset{C_{i}^{*}}{\stackrel{s}{\rightarrow}} T \Leftrightarrow\left\|T_{n}-T\right\|_{\theta} \rightarrow 0$.

Remember, by definition, every $T \in C_{i}(X, K)^{*}$, is linear and $[i] \tau_{s^{-}}$continuous. Since not every $K$-valued $[i] \tau_{s}$-continuous function should be linear, $[i] \tau_{s}$ may be a larger topology on $C_{i}(X, K)$ than what would be strictly necessary in order to make every $T \in C_{i}(X, K)^{*}$ continuous. This observation motivates the next topology to be introduced.

(2) weak topology on $C_{i}(X, K)$ : is the topology $[i] \tau_{w}$ defined as the coarsest topology on $C_{i}(X, K)$ that makes every $T \in C_{i}(X, K)^{*}$ continuous: $[i] \tau_{w}=\left\{T^{-1}(U): U \subset K, U\right.$ open, $T \in$ 
$\left.C_{i}(X, K)^{*}\right\}$, having $\left\{U_{f_{0}, T, \epsilon}: f_{0} \in C_{i}(X, K), \epsilon>0, T \in C_{i}(X, K)^{*}\right\}$ as a subbasis, where $U_{f_{0}, T, \epsilon}=$ $\left\{f \in C_{i}(X, K):\left|T(f)-T\left(f_{0}\right)\right|<\epsilon\right\}$.

(ii) weak convergence in $C_{i}(X, K): f_{n} \underset{C_{i}}{\stackrel{w}{\rightarrow}} f \Leftrightarrow \forall T \in C_{i}(X, K)^{*}, \epsilon>0, \exists N=N(T, \epsilon) \geq 0$, such that $\forall n \geq N, f_{n} \in U_{f, T, \epsilon} \Leftrightarrow \forall T, \epsilon, \exists N=N(T, \epsilon)$, such that $\forall n \geq N,\left|T\left(f_{n}\right)-T(f)\right|<\epsilon \Leftrightarrow T\left(f_{n}\right) \rightarrow$ $T(f), \forall T \in C_{i}(X, K)^{*}$.

$\left(2^{*}\right)$ weak topology on $C_{i}(X, K)^{*}$ : is the topology [i] $\tau_{w}^{*}$ defined as the coarsest topology that makes (only) every $S \in C_{i}(X, K)^{* *}$ to become continuous, where $C_{i}(X, K)^{* *}=\left\{S: C_{i}(X, K)^{*} \rightarrow K\right.$; linear and $\left.\|\cdot\|_{\theta^{-c o n t i n u o u s}}\right\}:[i] \tau_{w}^{*}=\left\{S^{-1}(U): U \subset K, U\right.$ open, $\left.S \in C_{i}(X, K)^{* *}\right\}$, having $\left\{U_{T_{0}, S, \epsilon}: T_{0} \in\right.$ $\left.C_{i}(X, K)^{*}, \epsilon>0, S \in C_{i}(X, K)^{* *}\right\}$ as a subbasis, where $U_{T_{0}, S, \epsilon}=\left\{T \in C_{i}(X, K)^{*}:\left|S(T)-S\left(T_{0}\right)\right|<\right.$ $\epsilon\}$.

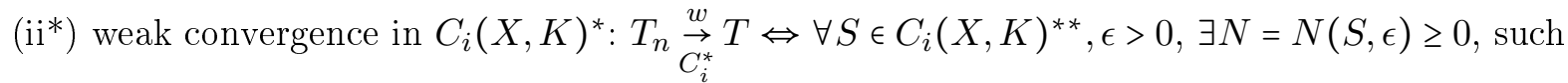
that $\forall n \geq N, T_{n} \in U_{T, S, \epsilon} \Leftrightarrow \forall S, \epsilon, \exists N=N(S, \epsilon)$, such that $\forall n \geq N,\left|S\left(T_{n}\right)-S(T)\right|<\epsilon \Leftrightarrow S\left(T_{n}\right) \rightarrow$ $S(T), \forall S \in C_{i}(X, K)^{* *}$.

(3) weak* topology on $C_{i}(X, K)$ : is the topology $[i] \tau_{w *}$ defined as the coarsest topology that makes (only) $T_{x} \in C_{i}(X, K)^{*}, T_{x}: g \in C_{i}(X, K) \mapsto g(x) \in K$, to become continuous, for all $x \in X$ : $[i] \tau_{w *}=\left\{T_{x}^{-1}(U): U \subset K, U\right.$ open, $\left.x \in X\right\}$, having $\left\{U_{f_{0}, x, \epsilon}: f_{0} \in C_{i}(X, K), \epsilon>0, x \in X\right\}$ as a subbasis, where $U_{f_{0}, x, \epsilon}=\left\{f \in C_{i}(X, K):\left|T_{x}(f)-T_{x}\left(f_{0}\right)\right|<\epsilon\right\}=\left\{f \in C_{i}(X, K):\left|f(x)-f_{0}(x)\right|<\epsilon\right\}$.

(iii) weak* convergence in $C_{i}(X, K): f_{n} \underset{C_{i}}{\overrightarrow{w *}} f \Leftrightarrow \forall x \in X, \epsilon>0, \exists N=N(x, \epsilon) \geq 0$, such that $\forall n \geq N, f_{n} \in U_{f, x, \epsilon} \Leftrightarrow \forall x, \epsilon, \exists N=N(x, \epsilon)$, such that $\forall n \geq N,\left|T_{x}\left(f_{n}\right)-T_{x}(f)\right|=\left|f_{n}(x)-f(x)\right|<\epsilon \Leftrightarrow$ $f_{n}(x) \rightarrow f(x), \forall x \in X$, i.e., it is only pointwise convergence.

$\left(3^{*}\right)$ weak* topology on $C_{i}(X, K)^{*}$ : is the topology $[i] \tau_{w *}^{*}$ defined as the coarsest topology that makes (only) $S_{f} \in C_{i}(X, K)^{* *}, S_{f}: T \in C_{i}(X, K)^{*} \mapsto T(f) \in K$, to become continuous, for all $f \in C_{i}(X, K):[i] \tau_{w *}^{*}=\left\{S_{f}^{-1}(U): U \subset K, U\right.$ open, $\left.f \in C_{i}(X, K)\right\}$, having $\left\{U_{T_{0}, f, \epsilon}: T_{0} \in\right.$ $\left.C_{i}(X, K)^{*}, \epsilon>0, f \in C_{i}(X, K)\right\}$ as a subbasis, where $U_{T_{0}, f, \epsilon}=\left\{T \in C_{i}(X, K)^{*}:\left|S_{f}(T)-S_{f}\left(T_{0}\right)\right|<\right.$ $\epsilon\}=\left\{T \in C_{i}(X, K)^{*}:\left|T(f)-T_{0}(f)\right|<\epsilon\right\}$.

(iii $^{*}$ ) weak* convergence in $C_{i}(X, K)^{*}: T_{n} \underset{C_{i}^{*}}{\stackrel{w *}{\rightarrow}} T \Leftrightarrow \forall f \in C_{i}(X, K), \epsilon>0, \exists N=N(S, \epsilon) \geq 0$, such that $\forall n \geq N, T_{n} \in U_{T, f, \epsilon} \Leftrightarrow \forall f, \epsilon, \exists N=N(f, \epsilon)$, such that $\forall n \geq N,\left|S_{f}\left(T_{n}\right)-S_{f}(T)\right|=\left|T_{n}(f)-T(f)\right|<$ $\epsilon \Leftrightarrow T_{n}(f) \rightarrow T(f), \forall f \in C_{i}(X, K)$.

Considering the above definitions, it is natural that $[i] \tau_{w *} \subset[i] \tau_{w} \subset[i] \tau_{s}$ and $[i] \tau_{w *}^{*} \subset[i] \tau_{w}^{*} \subset$ $[i] \tau_{s}^{*}$.

Each topology (convergence) on $C_{i}(X, K)^{*}, i \in\{0, c\}$, induces, through $\Phi$ and $\Gamma$, respectively, a topology (convergence) on $\mathcal{M}_{r}(X, K)$.

Below we present the induced topologies (convergences) and some additional definitions. In the following items, we denote $\left.T_{\mu}\right|_{C_{0}}: C_{0}(X, K) \rightarrow K$, given by $\left.T_{\mu}\right|_{C_{0}}(f)=\int f d \mu$, and $\left.T_{\mu}\right|_{C_{c}}$ : $C_{c}(X, K) \rightarrow K$, given by $\left.T_{\mu}\right|_{C_{c}}(f)=\int f d \mu$. These are precisely the images of $\mu$ in $C_{0}(X, K)^{*}$ and $C_{c}(X, K)^{*}$ under the appropriate isomorphisms ( $\Phi$ and $\Gamma$, respectively).

(1) strong (or uniform) topology on $\mathcal{M}_{r}(X, K)$ : is the topology $\mathcal{T}_{s}$ induced by the norm $\|\cdot\|_{m}$. Equivalently, $\mathcal{T}_{s}$ is also $\left\{\Phi^{-1}(U), U \in[0] \tau_{s}^{*}\right\}$ and $\left\{\Gamma^{-1}(U), U \in[c] \tau_{s}^{*}\right\}$.

(i) strong (or uniform) convergence in $\mathcal{M}_{r}(X, K): \mu_{n} \stackrel{s}{\rightarrow} \mu \Leftrightarrow\left\|\mu_{n}-\mu\right\|_{m} \rightarrow 0 \Leftrightarrow \|\left. T_{\mu_{n}}\right|_{C_{0}}-$ $\left.\left.T_{\mu}\right|_{C_{0}}\left\|_{\theta} \rightarrow 0 \Leftrightarrow\right\| T_{\mu_{n}}\right|_{C_{c}}-\left.T_{\mu}\right|_{C_{c}} \|_{\theta} \rightarrow 0$.

All the equivalences in (1) and (i) are because $\left(\mathcal{M}_{r}(X, K),\|\cdot\|_{m}\right),\left(C_{0}(X, K)^{*},\|\cdot\|_{\theta}\right)$ and $\left(C_{c}(X, K)^{*}\right.$, $\left.\|\cdot\|_{\theta}\right)$ are pairwise isomorphic.

The next topologies we describe twofold in each item, since our encapsulated notation define two (different) topologies at once.

(2) $C_{i}$ weak topology on $\mathcal{M}_{r}(X, K)$, for $i \in\{0, c\}$ : is the topology $[i] \mathcal{T}_{w}=\left\{\Upsilon_{i}^{-1}(U), U \in[i] \tau_{w}^{*}\right\}$, where $\Upsilon_{0}=\Phi, \Upsilon_{c}=\Gamma$, having $\left\{U_{\mu, S, \epsilon}: \mu \in \mathcal{M}_{r}(X, K), S \in C_{i}(X, K)^{* *}, \epsilon>0\right\}$ as a subbasis, where $U_{\mu, S, \epsilon}=\left\{\tilde{\mu} \in \mathcal{M}_{r}(X, K):\left|S\left(\left.T_{\tilde{\mu}}\right|_{C_{i}}\right)-S\left(\left.T_{\mu}\right|_{C_{i}}\right)\right|<\epsilon\right\}$.

(ii) $C_{i}$ weak convergence in $\mathcal{M}_{r}(X, K)$, for $i \in\{0, c\}: \mu_{n} \stackrel{w_{i}}{\longrightarrow} \mu \Leftrightarrow \forall S \in C_{i}(X, K)^{* *}, \epsilon>0, \exists N=$ $N(S, \epsilon)$, such that $\forall n \geq N, \mu_{n} \in U_{\mu, S, \epsilon} \Leftrightarrow \forall S, \epsilon, \exists N=N(S, \epsilon)$, such that $\forall n \geq N, \mid S\left(\left.T_{\mu_{n}}\right|_{C_{i}}\right)-$ 
$S\left(\left.T_{\mu}\right|_{C_{i}}\right) \mid<\epsilon \Leftrightarrow S\left(\left.T_{\mu_{n}}\right|_{C_{i}}\right) \rightarrow S\left(\left.T_{\mu}\right|_{C_{i}}\right), \forall S \in C_{i}(X, K)^{* *}$.

(3) $C_{i}$ weak* topology on $\mathcal{M}_{r}(X, K)$, for $i \in\{0, c\}$ : is the topology $[i] \mathcal{T}_{w} *=\left\{\Upsilon^{-1}(U), U \in\right.$ $\left.[i] \tau_{w *}^{*}\right\}$, where $\Upsilon_{0}=\Phi, \Upsilon_{c}=\Gamma$, having $\left\{U_{\mu, f, \epsilon}: \mu \in \mathcal{M}_{r}(X, K), f \in C_{i}(X, K), \epsilon>0\right\}$ as a subbasis, where $U_{\mu, f, \epsilon}=\left\{\tilde{\mu} \in \mathcal{M}_{r}(X, K):\left|T_{\tilde{\mu}}\right|_{C_{i}}(f)-\left.T_{\mu}\right|_{C_{i}}(f) \mid<\epsilon\right\}=\left\{\tilde{\mu} \in \mathcal{M}_{r}(X, K):\left|\int f d \tilde{\mu}-\int f d \mu\right|<\epsilon\right\}$.

(iii) $C_{i}$ weak* convergence in $\mathcal{M}_{r}(X, K)$, for $i \in\{0, c\}: \mu_{n} \underset{i}{\stackrel{w *}{\rightarrow}} \mu \Leftrightarrow \forall f \in C_{i}(X, K), \epsilon>0, \exists N=$ $N(f, \epsilon)$, such that $\forall n \geq N, \mu_{n} \in U_{\mu, f, \epsilon} \Leftrightarrow \forall f, \epsilon, \exists N=N(f, \epsilon)$, such that $\forall n \geq N,\left|\int f d \mu_{n}-\int f d \mu\right|<$ $\epsilon \Leftrightarrow \int f d \mu_{n} \rightarrow \int f d \mu, \forall f \in C_{i}(X, \mathbb{R})$.

Below, we leverage on the above findings to construct an analogous topology (convergence) using $C_{b}(X, K)$ instead of $C_{0}(X, K)$ or $C_{c}(X, K)$. We will omit the weak* designation below because, even though $C_{b}(X, K)^{*}$ itself has a weak* topology which is connected to the following topology, this connection is not in the form of a natural $\left(\|\cdot\|_{m},\|\cdot\|_{\theta}\right)$-isomorphism, as before. At this level of generality $\left(X\right.$ only LCH), not even a meaningful bijection between $\mathcal{M}_{r}(X, K)$ and $C_{b}(X, K)^{*}$ can be obtained (the natural choice $\left.\mu \mapsto T_{\mu}\right|_{C_{C}}$ would not be well defined since $\int f d \mu$ can be not in $K$ for many (non finite) $\mu$ 's and (non vanishing at infinity) $f$ 's).

(3') $C_{b}$ topology on $\mathcal{M}_{r}(X, K)$ : is the topology [b] $\mathcal{T}$ whose subbasis consists of subsets the form $U_{\mu, f, \epsilon}=\left\{\tilde{\mu} \in \mathcal{M}_{r}(X, K):\left|\int f d \tilde{\mu}-\int f d \mu\right|<\epsilon\right\}$, for $\mu \in \mathcal{M}_{r}(X, K), f \in C_{c}(X, K), \epsilon>0$.

(iii') $C_{b}$ convergence in $\mathcal{M}_{r}(X, K): \mu_{n} \underset{b}{\rightarrow} \mu \Leftrightarrow \int f d \mu_{n} \rightarrow \int f d \mu, \forall f \in C_{b}(X, K)$. The development of this equivalence identical to (iii), changing $C_{i}(X, K)$ by $C_{b}(X, K)$.

For our convenience, in the last characterization of (iii) and (iii'), with no loss of generality, we take as the set in which to test convergence, $C_{i}(X, \mathbb{R})$ instead of $C_{i}(X, K), i \in\{b, 0, c\}$. That is no issue, since, $C_{i}(X, \mathbb{R})$ constitutes a basis for $C_{i}(X, \mathbb{C})$, when we are allowed to use complex scalars.

(iv) pointwise convergence in $\mathcal{M}_{r}(X, K): \mu_{n} \stackrel{p}{\rightarrow} \mu \Leftrightarrow \mu_{n}(A) \rightarrow \mu(A)$, for every measurable $A$.

There are some subsets of $\mathcal{M}_{r}(X, K), K \in\{\mathbb{C}, \mathbb{R}\}$, that we highlight: $\mathcal{M}_{1, r}(X)=\left\{\mu \in \mathcal{M}_{r}(X, K)\right.$ : $\mu$ Borelian probability measure $\}$ and $\mathcal{M}_{1}(M)=\{\mu$ Borelian positive probability measure on $M\}$, being $M$ a metric space (every finite Borelian measure on a metric space is regular, hence Radon). Every subset of $\mathcal{M}_{r}(X, K)$ inherits each of the topologies (convergences) defined above. The sets $\mathcal{M}_{r}(X,[0, \infty])$ and $\mathcal{M}_{r}(X,[-\infty, \infty])$ are not a subsets of $\mathcal{M}_{r}(X, K)$ (because they include $\infty$ ), so the specific characterizations above will not be inherited, but, with caution, can be extrapolated.

Although of great interest for us, as defined and at the level of generality we are working with

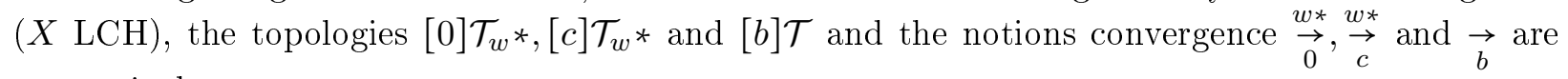
not equivalent.

We could discuss under which conditions those objects, restricted to the space of probability measures, are equivalent. However we will not carry out this analysis, we will simply consider from now on that $X$ is a compact metric space, denoted by $X=\bar{M}$.

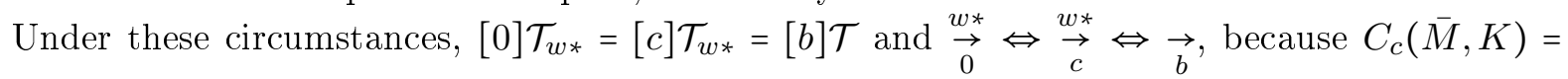
$C_{0}(\bar{M}, K)=C_{b}(\bar{M}, K)=C(\bar{M}, K)$, the set of $K$-valued continuous functions on $\bar{M}$. They are, respectively, the so called weak* topology and convergence (denoted $\stackrel{w *}{\rightarrow}$ ). We are specially interested in the case in which they are restricted to the space of probability measures.

Next we explore some properties of the weak* restricted to the space of probability measures, weak $\left.*\right|_{\mathcal{M}_{1}(\bar{M})}$.

The following proposition is used to show its metrizability and is useful for its own sake, as it richly characterizes the weak* topology/convergence.

Proposition 2.2.1 (Portmanteau). Let $\bar{M}$ be compact metric space. Then the weak $*$ topology on $\mathcal{M}_{1}(\bar{M})$ is equivalent to that having the following neighborhood basis:

(i) $V_{\mu, \mathbf{f}, \epsilon}=\left\{\tilde{\mu} \in \mathcal{M}_{1}(\bar{M}):\left|\int f_{j} d \tilde{\mu}-\int f_{j} d \mu\right|<\epsilon, j=0, \ldots, n\right\}$, with $\mathbf{f}=\left\{f_{0}, \ldots, f_{n}\right\}$ being a finite family of Lipschitz functions.

(ii) $V_{\mu, \mathbf{H}, \epsilon}=\left\{\tilde{\mu} \in \mathcal{M}_{1}(\bar{M}): \tilde{\mu}\left(H_{j}\right)<\mu\left(H_{j}\right)+\epsilon, j=0, \ldots, n\right\}$, with $\mathbf{H}=\left\{H_{0}, \ldots, H_{n}\right\}$ being a finite family of closed sets.

(iii) $V_{\mu, \mathbf{U}, \epsilon}=\left\{\tilde{\mu} \in \mathcal{M}_{1}(\bar{M}): \tilde{\mu}\left(U_{j}\right)>\mu\left(U_{j}\right)-\epsilon, j=0, \ldots, n\right\}$, with $\mathbf{U}=\left\{U_{0}, \ldots, U_{n}\right\}$ being a finite family of open sets. 
(iv) $V_{\mu, \mathbf{C}, \epsilon}=\left\{\tilde{\mu} \in \mathcal{M}_{1}(\bar{M}):\left|\tilde{\mu}\left(C_{j}\right)-\mu\left(C_{j}\right)\right|<\epsilon, j=0, \ldots, n\right\}$, with $\mathbf{C}=\left\{C_{0}, \ldots, C_{n}\right\}$ being a finite family of $\mu$-continuous sets, i.e., such that $\mu\left(\partial C_{j}\right)=0$.

Consequently, if $\left(\mu_{n}\right)_{n \geq 0}$ and $\mu$ are in $\mathcal{M}_{1}(\bar{M})$, then $\mu_{n} \stackrel{w *}{\longrightarrow} \mu$ if, and only if:

(i) $\lim \int f d \mu_{n}=\int d \mu$, for all $f: X \rightarrow \mathbb{R}$ Lipschitz

(ii) $\lim \sup \mu_{n}(H) \leq \mu(H)$, for all closed $H$

(iii) $\liminf \mu_{n}(U) \geq \mu(U)$, for all open $U$

(iv) $\lim \mu_{n}(C)=\mu(C)$, for all $\mu$-continuous $C$.

The following result is only stated and presented together with its associated metric. The details of the proof are not important here, but can be found on [VO16].

Proposition 2.2.2 (The weak* topology on $\mathcal{M}_{1}(\bar{M})$ is metrizable). Let $\bar{M}$ be a compact metric space. Then $e(\nu, \mu)=\inf \left\{\delta>0: \nu(B) \leq \mu\left(B^{\delta}\right)+\delta\right.$ and $\mu(B) \leq \nu\left(B^{\delta}\right)+\delta$, for all measurable $\left.B\right\}$, where $B^{\delta}=\{\tilde{m} \in \bar{M}: \exists m \in B$ such that $\|\tilde{m}-m\|<\delta\}$, defines a metric (Levy-Prohorov metric) on $\mathcal{M}_{1}(\bar{M})$ whose induced topology is equivalent to the weak* topology.

In fact, this metric serves in a more general context. When dealing with a compact metric space $\bar{M}$, other simpler metrics are available, such as $e(\nu, \mu)=\sum_{j \in \mathbb{N}} 2^{-j}\left|\int f_{j} d \nu-\int f_{j} d \mu\right|$, where $\left\{f_{j}: j \in \mathbb{N}\right\}$ is a countable dense subset of $C(\bar{M}, K)$ (available due to the nature of $\bar{M}$ ).

Finally, we discuss the last result regarding the weak* topology on $\mathcal{M}_{1}(M)$.

Proposition 2.2.3 (Weak* and compactness). Let $\bar{M}$ be a compact metric space and $\mathcal{M}_{1}(\bar{M})$ be equipped with the weak* topology. Then $\left(\mathcal{M}_{1}(\bar{M})\right.$, weak*) is a compact topology.

Since $\mathcal{M}_{1}(\bar{M})$ is metric space, the result is accomplished showing it is sequentially compact. For such let $\left(\mu_{n}\right)_{n \geq 0}$ be in $\mathcal{M}_{1}(\bar{M})$ and, knowing $\bar{M}$ is compact makes $C(\bar{M})=C_{b}(\bar{M})$ separable. Take an enumerable $\|\cdot\|_{u}$-dense set $\left\{f_{j}: j \geq 0\right\}$ in $\{f \in C(\bar{M}):\|f\| \leq 1\}$. For each $j$, note that $\int f_{j} d \mu_{n} \in[0,1]$. For each $j$, find a subsequence $n_{k}^{j}$ that makes $\int f_{j} d \mu_{n_{k}^{j}}$ convergent, say, to $a_{j}$. By choosing subsequences adequately, we can end with an only subsequence $n_{l}$ along which, for every $j, \int f_{j} d \mu_{n_{l}}$ still converges to $a_{j}$. That defines a function $\alpha: f \in C(\bar{M}) \mapsto \lim _{l} f d \mu_{n_{l}} \in \mathbb{R}$, assuming value $a_{j}$ on $f_{j}$, but also well defined elsewhere. For instance, for $f \in C(\bar{M})$ such that $\|f\| \leq 1$, choose $f_{j}$ such that $\left\|f-f_{j}\right\|_{u}<\epsilon$, than $\left|\int f d \mu_{n_{l}}-\int f_{j} d \mu_{n_{l}}\right|<\epsilon, \forall j \geq 0 \Rightarrow \int f_{j} d \mu_{n_{l}}-$ $\epsilon \leq \int f d \mu_{n_{l}} \leq \int f_{j} d \mu_{n_{l}}+\epsilon \Rightarrow \int f_{j} d \mu_{n_{l}}-\epsilon \leq \liminf _{j} \int f d \mu_{n_{l}} \leq \limsup _{j} \int f d \mu_{n_{l}} \leq \int f_{j} d \mu_{n_{l}}+\epsilon \Rightarrow$ $\limsup _{j} \int f d \mu_{n_{l}}-\liminf _{j} \int f d \mu_{n_{l}} \leq 2 \epsilon$, then $\int f d \mu_{n_{l}}$ converges as $l \rightarrow \infty$. When $f \in C(\bar{M})$ and $\|f\|_{u}>1$, we scale it by $1 /\|f\|_{u}$ and use linearity. Finally, $\alpha$ is linear by definition, positive $(f \geq 0$, so integration against $\mu_{n_{l}}$ are positive as well as its limit) and $\alpha(1)=1$ (integration against $\mu_{n_{l}}$ is 1 as well as its limit). Then Riesz-Markov representation theorem says $\alpha=\int \cdot d \mu$, for some $\mu \in \mathcal{M}_{1}(\bar{M})$. Therefore $\int f d \mu=\lim _{l} \int f d \mu_{n_{l}}, \forall f \in C(\bar{M})$, thus $\mu_{n_{l}} \stackrel{w *}{\longrightarrow} \mu$ as $l \rightarrow \infty$.

\subsection{Probability Theory}

The basic object of study of probability theory is a probability measure space $(X, \mathcal{A}, \mu)$. During this section, this triple, but especially the probability measure $\mu$, will be a sort of primitive object and can be understood as fixed for all purposes.

As in section 2.1, when $X$ is a topological space, unless otherwise stated, we assume $X$ is equipped with the Borel $\sigma$-algebra, already introduced.

Rigorously and abstractly speaking, objects of probability theory are a special case of those introduced in section 2.1. But probabilists' attitude towards them may be different, so the nomenclature and notation adopted by them is distinct and supposed to reflect this attitude.

Not willing to understate the importance of notation, we will, from the beginning, unify notation and stick to the analysts' style. On the other hand, while introducing basic probabilistic concepts, we will invest some words trying to explain that attitude so the reader can bring all that intuition together. 
First of all, the probabilistic interpretation of a probability measure space $(X, \mathcal{A}, \mu)$ herein adopted is that it models a random experiment (i.e., an experiment whose outcome can not be told in advance), where:

(a) $X$, called 'sample space', is the set of all (virtually) possible outcomes (whose elements $x$ are called 'outcomes'), and

(b) one wants to know whether the outcome of such experiment belongs to a given subset $A \subset X$, but not every $A \subset X$ is "distinguished enough" (or non-pathological enough) to be of concern, then

(c) the only sets to be evaluated are those $A \in \mathcal{A}$, which are called 'events' (and $\mathcal{A}$ is called 'set of events'), to which

(d) the probability measure $\mu$ assigns a value between 0 and 1 , to be understood as the probability (here in common sense meaning) that the experiment outcome belongs to a given $A \in \mathcal{A}$.

The ideas introduced above should be kept, but the nomenclature will not be used.

Other important objects are measurable functions $f:(X, \mathcal{A}) \rightarrow(Y, \mathcal{B})$. We will keep using lowercase Greek letters such as $\psi$ and $\varphi$ for these functions, specially for those at the center of our analysis. We will permit ourselves to use lowercase Latin letter such as $f, g$ and $h$ for auxiliary measurable functions in specific contexts - use will make it clear (ignore the fact that they will be used to denote dynamical systems in section 2.4). These measurable functions will generally (but not necessarily) take values in a space with a standard structure, such as $\mathbb{N}, \mathbb{R}, \overline{\mathbb{R}}, \mathbb{C}$ (for which an integration theory was developed in section 2.1) or in their cartesian products.

During this section, if a measurable function is to take values in one of those standard spaces above, by default, it will take values in $\overline{\mathbb{R}}^{e}, e \in \mathbb{N} \cup\{\infty\}$.

Probabilists call $\overline{\mathbb{R}}^{e}$-valued measurable functions by random variables. Random variables (i) reduce the space $X$ into an (usually) less complicated space that registers just what probabilists are interested in (e.g., the domain $X$ can be the set of triangles, the codomain, $\overline{\mathbb{R}}$, and the measurable function that assigning each triangle to its area) and (ii) have enough quality (i.e., measurability) so that, for example, the probability measure on $(X, \mathcal{A})$ can flow into $\left(\overline{\mathbb{R}}^{e}, \mathcal{B}_{\overline{\mathbb{R}}}\right)$, in the sense we make precise below.

In this section, we use random variables and $\overline{\mathbb{R}}^{e}$-valued measurable functions interchangeably. In chapter 4, we will use random variables to emphasize probabilistic aspects of the mathematical structure to be used.

Definition 2.3.1 (Distribution measure of a measurable function). Let $(X, \mathcal{B}, \mu)$ be a probability measure space and $\psi:(X, \mathcal{B}) \rightarrow\left(\overline{\mathbb{R}}^{e}, \otimes_{1}^{e} \mathcal{B}_{\overline{\mathbb{R}}}=\mathcal{B}_{\overline{\mathbb{R}}^{e}}\right)$ be a measurable function.

The distribution measure of $\psi$, or the pushforward/image measure of $\mu$ through $\psi$, is the probability measure $\psi_{\star} \mu=: \nu$ on $\left(\overline{\mathbb{R}}^{e}, \bigotimes_{1}^{e} \mathcal{B}_{\overline{\mathbb{R}}}=\mathcal{B}_{\overline{\mathbb{R}}^{e}}\right)$, defined as $\psi_{\star} \mu(B)=\mu\left(\psi^{-1}(B)\right), \forall B \in \bigotimes_{1}^{e} \mathcal{B}_{\overline{\mathbb{R}}}=\mathcal{B}_{\overline{\mathbb{R}}^{e}}$.

Of course different random variables can share the same distribution: $X=\{0,1\}, \mu(\{0\}), \mu(\{1\})=$ $1 / 2, \psi(x)=x, \varphi(x)=-x+1, \psi_{*} \mu(\{0\}), \varphi_{*} \mu(\{0\})=1 / 2, \psi_{*} \mu(\{1\}), \varphi_{*} \mu(\{1\})=1 / 2$, then $\psi$ and $\varphi$ are everywhere different but have the same distribution.

It is worth repeating: a distribution measure $\psi_{\star} \mu=\nu$ on $\left(\overline{\mathbb{R}}, \mathcal{B}_{\overline{\mathbb{R}}}\right)$, or a probability measure $\nu$ on $\left(\overline{\mathbb{R}}, \mathcal{B}_{\overline{\mathbb{R}}}\right)$, induces its so called cumulative distribution function $F$, defined by $x \in \overline{\mathbb{R}} \mapsto F(x):=$ $\nu((-\infty, x]) \in[0,1]$. By theorem 2.1.13, starting with one such $F$, one can uniquely determine the probability measure $\nu$ whose cumulative distribution function is $F$.

One can generalize cumulative distribution functions to distribution measures $\psi_{*} \mu=\nu$ on $\left(\overline{\mathbb{R}}^{e}, \bigotimes_{1}^{e} \mathcal{B}_{\overline{\mathbb{R}}}=\mathcal{B}_{\overline{\mathbb{R}}^{e}}\right)$, or to probability measures $\nu$ on $\left(\overline{\mathbb{R}}^{e}, \bigotimes_{1}^{e} \mathcal{B}_{\overline{\mathbb{R}}}=\mathcal{B}_{\overline{\mathbb{R}}^{e}}\right)$, by letting be $F: x \in \overline{\mathbb{R}}^{e} \mapsto$ $F(x):=\nu((-\infty, x]) \in[0,1]$, where $(-\infty, x]$ is the set of vectors in $\overline{\mathbb{R}}^{e}$ which are component-wise $\leq x$. The reason we were allowing for the possibility that $e$ is greater than 1 shows up next.

A great emphasis is devoted to families of $\overline{\mathbb{R}}$ valued measurable functions $\left(\psi_{\alpha}:(X, \mathcal{A}) \rightarrow\right.$ $\left.\left(\overline{\mathbb{R}}, \mathcal{B}_{\overline{\mathbb{R}}}\right)\right)_{\alpha \in \Lambda}$, from which one can derive a joint measurable function $\otimes_{\alpha \in \Lambda} \psi_{\alpha}:(X, \mathcal{A}) \rightarrow\left(\prod_{\alpha \in \Lambda} \overline{\mathbb{R}}\right.$ $\left.\bigotimes_{\alpha \in \Lambda} \mathcal{B}_{\overline{\mathbb{R}}}\right)$ mapping $x \mapsto\left(\psi_{\alpha}(x)\right)_{\alpha \in \Lambda}$. The measurability of $\otimes_{\alpha \in \Lambda} \psi_{\alpha}$ follows from a corollary of 2.1.34, since each coordinate function of $\otimes_{\alpha \in \Lambda} \psi_{\alpha}, p r_{\alpha} \circ \bigotimes_{\alpha \in \Lambda} \psi_{\alpha}=\psi_{\alpha}$, is measurable. Reducing to the case when $\Lambda=\{0,1, \ldots, e-1\}$ is finite $(e \in \mathbb{N})$ or $\Lambda=\mathbb{N}$ is countable (" $e-1=\infty$ and $e=\infty$ "), we derive the joint measurable function $\bigotimes_{j=0}^{e-1} \psi_{j}:(X, \mathcal{A}) \rightarrow\left(\overline{\mathbb{R}}^{e}, \bigotimes_{j=0}^{e-1} \mathcal{B}_{\overline{\mathbb{R}}}=\mathcal{B}_{\mathbb{R}^{e}}\right)$, whose distribution can be obtained as shown above, by letting be $\psi:=\bigotimes_{j=0}^{e-1} \psi_{j}$. 
With the notation above, the following diagram summarizes some of the objects already discussed.

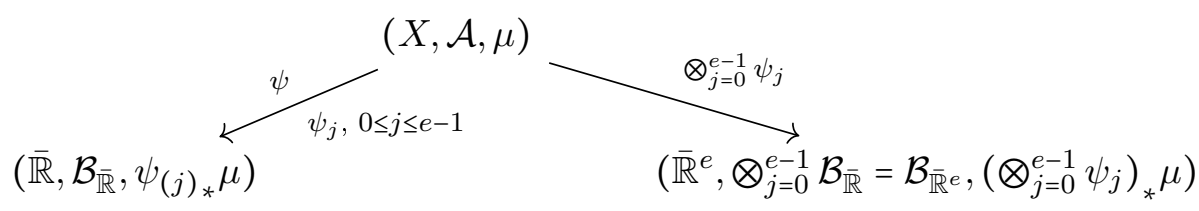

A family of $\overline{\mathbb{R}}$ valued measurable functions $\left(\psi_{\alpha}\right)_{\alpha \in \Lambda}$ is said to be $\mu$-identically distributed (or, omitting $\mu$, identically distributed) if $\psi_{\alpha_{*}} \mu=\nu, \forall \alpha \in \Lambda$.

We will now focus on the notion of independence. As always, $(X, \mathcal{A}, \mu)$ still fixed.

Independence is first defined in all of its generality for an arbitrary collection $\left(\mathcal{B}_{\alpha}\right)_{\alpha \in \Lambda}$ of $\sigma$ algebras on $X$ contained in $\mathcal{A}$. But a $\sigma$-algebra $\mathcal{B}$ always comes in hand with the set of functions that are measurable with respect to it, $\mathcal{F}_{\mathcal{B}}=\left\{f:(X, \mathcal{B}) \rightarrow\left(\overline{\mathbb{R}}, \mathcal{B}_{\overline{\mathbb{R}}}\right) ; f\right.$ is measurable $\}$ (the codomain was an illustrative choice), and vice versa, a set $\mathcal{F}$ of functions always come in hand with a $\sigma$ algebra, that $\sigma$-algebra induced by the family $\mathcal{F}$, already defined in section 2.1 , here denoted $\mathcal{B}_{\mathcal{F}}$ or $\sigma(\mathcal{F})$. We will make use of this connection to leverage on our coming definition of independence for families of $\sigma$-algebra $\left(\mathcal{B}_{\alpha}\right)_{\alpha \in \Lambda}$ so that families of set of functions $\left(\mathcal{F}_{\alpha}\right)_{\alpha \in \Lambda}$ can also enjoy it.

First we show a technical result, characterizing which functions are measurable with respect to $\sigma(\mathcal{F})$.

Proposition 2.3.2. [Characterization of a $\sigma$-algebra generated by a family of functions] (i) Let $\psi:(X, \mathcal{A}) \rightarrow(Y, \mathcal{B})$ be a measurable function and $\mathcal{F}=\{\psi\}$. Then a function $g: X \rightarrow \overline{\mathbb{R}}$ belongs to $\sigma(\mathcal{F})$ if, and only if, there exists a measurable function $h:(Y, \mathcal{B}) \rightarrow \overline{\mathbb{R}}$, such that $g=h \circ \psi$.

(ii) Let $\mathcal{F}=\left(\psi_{n}:(X, \mathcal{A}) \rightarrow\left(Y_{n}, \mathcal{B}_{n}\right)\right)_{n \geq 0}$ be a family of measurable functions. Then a function $g:$ $X \rightarrow \overline{\mathbb{R}}$ belongs to $\sigma(\mathcal{F})$ if, and only if, there exists a measurable function $h:\left(\prod_{n \geq 0} Y_{n}, \otimes_{n \geq 0} \mathcal{B}_{n}\right) \rightarrow$ $\overline{\mathbb{R}}$, such that $g=h \circ \psi$. (follows from studying $\bigotimes_{n \geq 0} \psi_{n}$ in (i))

(iii) Let $\mathcal{F}=\left(\psi_{\alpha}(X, \mathcal{A}) \rightarrow\left(Y_{\alpha}, \mathcal{B}_{\alpha}\right)\right)_{\alpha \in \Lambda}$ be an arbitrary family of measurable functions. Then a function $g: X \rightarrow \overline{\mathbb{R}}$ belongs to $\sigma(\mathcal{F})$ if, and only if, there exists a sequence $\left(\alpha_{n}\right)_{n \geq 0}$ of elements in $\Lambda$ and a function $h:\left(\prod_{n \geq 0} Y_{\alpha_{n}}, \bigotimes_{n \geq 0} \mathcal{B}_{\alpha_{n}}\right) \rightarrow \overline{\mathbb{R}}$ such that $g=h \circ \psi$ (follows from studying $\bigotimes_{\alpha \in \Lambda} \psi_{\alpha}$ in (i), but some simplifications show up).

Now we address many core ideas regarding independence.

Definition 2.3.3 (Independent family of $\sigma$-algebras). Let $\left(\mathcal{B}_{j}\right)_{j=0}^{k-1}, k \in \mathbb{N}$, be a finite family of $\sigma$-algebras on $X$ contained in $\mathcal{A}$ and $\mathcal{F}_{\mathcal{B}_{j}}^{+}=\left\{f:\left(X, \mathcal{B}_{j}\right) \rightarrow\left([0, \infty], \mathcal{B}_{[0, \infty]}\right) ; f\right.$ is measurable $\}$. The family $\left(\mathcal{B}_{j}\right)_{j=0}^{k-1}$ is said to be $\mu$-independent (or independent) if:

$\int g_{0} \cdot \ldots \cdot g_{k-1} d \mu=\int g_{0} d \mu \cdot \ldots \cdot \int g_{k-1} d \mu, \forall g_{0} \in \mathcal{F}_{\mathcal{B}_{0}}^{+}, \ldots, g_{k-1} \in \mathcal{F}_{\mathcal{B}_{k-1}}^{+}$

An arbitrary family $\left(\mathcal{B}_{\alpha}\right)_{\alpha \in \Lambda}$ said to be $\mu$-independent (or independent) if every finite subfamily is independent.

Remark. Note the definition resorts to $\mathcal{F}_{\mathcal{B}}^{+}$. Also, $\mathcal{F}_{\mathcal{B}}^{+}$is preferred to $\mathcal{F}_{\mathcal{B}}$, presented above, to get it well defined.

A natural restatement of the definition is shown in the following proposition. An interpretation of a result analogous to this one is given later.

Proposition 2.3.4 (Checking independence of $\sigma$-algebras in a generating algebra). Let $\left(\mathcal{B}_{j}\right)_{j=0}^{k-1}$, $k \in \mathbb{N}$, be a finite family of $\sigma$-algebras on $X$ contained in $\mathcal{A}$ and $\left(\mathcal{B}_{j}\right)_{j=0}^{k-1}$ algebras generating the respective $\sigma$-algebras (one could even relax the generating set in which to check).

Then $\left(\mathcal{B}_{j}\right)_{j=0}^{k-1}$ is independent if, and only if, $\mu\left(B_{0} \cap \ldots \cap B_{k-1}\right)=\mu\left(B_{0}\right) \cdot \ldots \cdot \mu\left(B_{k-1}\right), \forall B_{0} \in$ $\mathcal{B}_{0}, \ldots, B_{k-1} \in \mathcal{B}_{k-1}$.

We now leverage on this definition.

Definition 2.3.5 (Independent family of measurable functions). Let $\left(\psi_{\alpha}:(X, \mathcal{A}) \rightarrow\left(Y_{j}, \mathcal{B}_{\alpha}\right)\right)_{\alpha \in \Lambda}$ be an arbitrary family of measurable functions and define $\mathcal{F}_{\alpha}:=\left\{\psi_{\alpha}\right\}$. The family is said to be $\mu$-independent (or independent) if the family of $\sigma$-algebras $\left(\sigma\left(\mathcal{F}_{\alpha}\right)\right)_{\alpha \in \Lambda}$ is independent. 
Generally speaking, a collection of objects is independent if the collection of $\sigma$-algebras generated by each of this objects is independent. For example, being:

(i) $\psi:(X, \mathcal{A}) \rightarrow(Y, \mathcal{B})$ a measurable function,

(ii) $\mathcal{C}$ a $\sigma$-algebra on $X$ contained in $\mathcal{A}$,

(iii) $\Phi=\left\{\varphi_{\alpha}:(X, \mathcal{A}) \rightarrow\left(Y_{\alpha}, \mathcal{B}_{\alpha}\right): \alpha \in \Lambda, \varphi_{\alpha}\right.$ a measurable function $\}$

(iv) $\mathcal{E}=\left\{\mathcal{D}_{\alpha}: \alpha \in \Lambda, \mathcal{D}_{\alpha}\right.$ a $\sigma$-algebra on $X$ contained in $\left.\mathcal{A}\right\}$,

we can say the family $\{\psi, \mathcal{C}, \Phi, \mathcal{E}\}$ is independent if the family of $\sigma$-algebras $\left\{\sigma_{1}, \sigma_{2}, \sigma_{3}, \sigma_{4}\right\}$ is independent, being $\sigma_{1}=\sigma(\{\psi\}), \sigma_{2}=\mathcal{C}, \sigma_{3}=\sigma(\Phi)$ and $\sigma_{4}=\sigma\left(\cup_{\alpha \in \Lambda} \mathcal{D}_{\alpha}\right)$, where this last $\sigma$ is the $\sigma$-algebra generated (not by a family of functions but) by a subset of $\mathcal{P}(X)$, introduced in section 2.1 .

In this case, since we know how to reduce (i)-(iv) to $\sigma$-algebras, objects in the previous paragraph may be understood as any of those in (i)-(iv) arranged or mixed in arbitrary collections, which themselves can recursively be arranged or mixed in arbitrary collections etc, up to finite recursion. The formalization of this issue seems not really important for our purposes.

Independence is preserved under groupings, e.g., if $\left\{\psi_{1}, \psi_{2}, \psi_{3}, \psi_{4}\right\}$ is an independent family, then $\left\{\boldsymbol{\psi}_{A}, \boldsymbol{\psi}_{B}\right\}$, where $\boldsymbol{\psi}_{A}=\left\{\psi_{1}, \psi_{2}\right\}$ and $\boldsymbol{\psi}_{B}=\left\{\psi_{3}, \psi_{4}\right\}$, is also an independent family.

Proposition 2.3.6 (Independence is preserved under groupings). Let $\left(\theta_{\alpha}\right)_{\alpha \in \Lambda}$ be an arbitrary family of independent objects, in the above sense, and $\dot{\cup}_{\beta \in B} \Delta_{\beta}=\Lambda$ be a partition of $\Lambda$ (whose components are non empty). Then the family $\left\{\theta^{\prime}=\cup_{\alpha \in \Delta_{\beta}}\left\{\theta_{\alpha}\right\}: \beta \in B\right\}$ is independent.

We now explore a characterization of independence that focus on measurable functions and provides some other intuitive results.

Proposition 2.3.7 (Independent family of measurable functions characterization). Let $\left(\psi_{j}:(X, \mathcal{A})\right.$ $\left.\rightarrow\left(Y_{j}, \mathcal{B}_{j}\right)\right)_{j=0}^{k-1}, k \in \mathbb{N}$, be a family of measurable functions. This family is $\mu$-independent (or independent) if, and only if, the joint measurable function $\hat{\psi}=\bigotimes_{j=0}^{k-1} \psi_{j}:(X, \mathcal{A}) \rightarrow\left(\prod_{j=0}^{k-1} X_{j}, \bigotimes_{j=0}^{k-1} \mathcal{B}_{j}\right)$ mapping $x \mapsto\left(\psi_{j}(x)\right)_{j=0}^{k-1}$ has (joint) distribution $\hat{\psi}_{*} \mu$ on the product space given by $\hat{\psi}_{*} \mu=\psi_{0_{*}} \mu \times$ $\ldots \times \psi_{k-1 *} \mu$. Since both these measures are defined on the (countable) product $\sigma$-algebra $\otimes_{j=0}^{k-1} \mathcal{B}_{j}$, generated by the set of rectangles $\mathcal{R}$, by propositions 2.1 .36 and Caratheodory's extension theorem, it suffices to check that

$$
\begin{array}{r}
\hat{\psi}_{*} \mu\left(\left(B_{0}, \ldots, B_{k-1}\right)\right)=\psi_{0 \star} \mu\left(B_{0}\right) \cdot \ldots \cdot \psi_{k-1 \star} \mu\left(B_{k-1}\right) \\
\Leftrightarrow \mu\left(\psi_{0}^{-1}\left(B_{0}\right) \cap \ldots \cap \psi_{k-1}^{-1}\left(B_{k-1}\right)\right)=\mu\left(\psi_{0}^{-1}\left(B_{0}\right)\right) \cdot \ldots \cdot \mu\left(\psi_{k-1}^{-1}\left(B_{k-1}\right)\right)
\end{array}
$$

, $\forall\left(B_{0}, \ldots, B_{k-1}\right)$, such that $B_{j} \in \mathcal{B}_{j}, 0 \leq j \leq k-1$ (or $B_{j} \in \mathcal{G}_{j}, 0 \leq j \leq k-1$, if each $\mathcal{G}_{j}$ generates $\mathcal{B}_{j}$ ).

It should be clear that independence is a property of measurable functions that can not be reduced to a property of their distributions, because it cares about how they are entangled, what can not be checked with distributions only. Nonetheless, we give a counterexample: $X=\{(0,0),(0,1)$, $(1,0)(1,1)\}, \mu(\{p\})=1 / 4, \forall p \in X$. Note $\psi(x, y)=x, \varphi(x, y)=y$ are independent. On the other hand $\psi^{\prime}(x)=x$ has the same distribution of $\psi$ and $\varphi^{\prime}(x, y)=x$ has the same distribution of $\varphi$, but $\psi^{\prime}$ and $\varphi^{\prime}$ are obviously not independent, they are the same.

An immediate consequence of the above proposition is the following.

Proposition 2.3.8 (Independence integral characterization). Let $(X, \mathcal{A}, \mu)$ be a measure space, $\left(\psi_{j}:(X, \mathcal{A}) \rightarrow\left(Y_{j}, \mathcal{B}_{j}\right)\right)_{j=0}^{k-1}, k \in \mathbb{N}$, be a finite family of measurable functions and $\hat{\psi}=\bigotimes_{j=0}^{k-1} \psi_{j}$ : $(X, \mathcal{A}) \rightarrow\left(\prod_{j=0}^{k-1} X_{j}, \otimes_{j=0}^{k-1} \mathcal{B}_{j}\right)$ mapping $x \mapsto\left(\psi_{j}(x)\right)_{j=0}^{k-1}$, then $\left(\psi_{j}\right)_{j=0}^{k-1}$ is independent if, and only if, for every family of measurable functions $\left(g_{j}:\left(Y_{j}, \mathcal{B}_{j}\right) \rightarrow\left(\overline{\mathbb{R}}, \mathcal{B}_{\overline{\mathbb{R}}}\right)\right)_{j=0}^{k-1}$, we have that $\int g_{0} \circ \psi_{0} d \mu \cdot \ldots$. $g_{k-1} \circ \psi_{k-1} d \mu=\int g_{0} \circ \psi_{0} d \mu \cdot \ldots \cdot \int g_{k-1} \circ \psi_{k-1} d \mu$, whenever every integral is well defined.

As a corollary, if a family of $\left(\psi_{j}\right)_{j=0}^{k-1}$ of $\overline{\mathbb{R}}$-valued measurable functions on $(X, \mathcal{A}, \mu)$ is independent, then $\int \psi_{0} \cdot \ldots \cdot \psi_{k-1} d \mu=\int \psi_{0} d \mu \cdot \ldots \cdot \int \psi_{k-1} d \mu$ (take $g_{j}=I d_{\overline{\mathbb{R}}}$ ), but that is not sufficient for independence, as it will be shown later when talking about correlation. We say that, for independent 
measurable functions, the average of the product equals the product of the average. To illustrate, if $g_{0}=\mathcal{X}_{A}$ and $g_{1}=\mathcal{X}_{B}$ are $(\mu-)$ independent, so $g_{0} \cdot g_{1}=\mathcal{X}_{A \cap B}$, then $\mu(A \cap B)=\mu(A) \mu(B)$; if the last two are non-zero, then $\mu(A \cap B) / \mu(A)=\mu(B)$, that is, the proportion of mass of $B$ within $A$ is equal to the overall proportion of mass of $B$ within the whole space (reversing $A$ and $B$ also holds, by symmetry).

Another corollary is that if $\left(\psi_{j}:(X, \mathcal{A}) \rightarrow\left(Y_{j}, \mathcal{B}_{j}\right)\right)_{j=0}^{k-1}$ is a family of independent measurable functions and $\left(h_{j}:\left(Y_{j}, \mathcal{B}_{j}\right) \rightarrow\left(Y_{j}, \mathcal{B}_{j}\right)\right)_{j=0}^{k-1}$ or, respectively, $\left(h_{j}:\left(Y_{j}, \mathcal{B}_{j}\right) \rightarrow\left(\overline{\mathbb{R}}, \mathcal{B}_{\overline{\mathbb{R}}}\right)\right)_{j=0}^{k-1}$, is a family of measurable functions, then the family $\left(h_{j} \circ \psi_{j}\right)_{j=0}^{k-1}$ is also independent: let $\left(g_{i}:\left(Y_{j}, \mathcal{B}_{j}\right) \rightarrow\right.$ $\left.\left(\overline{\mathbb{R}}, \mathcal{B}_{\overline{\mathbb{R}}}\right)\right)_{j=0}^{k-1}$ or, respectively, $\left.\left(g_{i}:\left(\overline{\mathbb{R}}, \mathcal{B}_{\overline{\mathbb{R}}}\right)\right) \rightarrow\left(\overline{\mathbb{R}}, \mathcal{B}_{\overline{\mathbb{R}}}\right)\right)_{j=0}^{k-1}$ be an arbitrary family of measurable functions, then, in either cases, $\left(g_{j} \circ h_{j}:\left(Y_{j}, \mathcal{B}_{j}\right) \rightarrow\left(\overline{\mathbb{R}}, \mathcal{B}_{\overline{\mathbb{R}}}\right)\right)_{j=0}^{k-1}$ is a family of measurable functions, so $\int g_{0} \circ h_{0} \circ \psi_{0} \cdot \ldots \cdot g_{k-1} \circ h_{k-1} \circ \psi_{k-1} d \mu=\int g_{0} \circ h_{0} \circ \psi_{0} d \mu \cdot \ldots \cdot \int g_{k-1} \circ h_{k-1} \circ \psi_{k-1} d \mu$, since $\left(g_{j}\right)_{j=0}^{k-1}$ was arbitrary, the result follows. We say independence is invariant under measurable composition. To illustrate, if $\psi_{0}, \psi_{1}$ and $\psi_{2}$ are independent, then $e^{\psi_{0}}, \max \left\{0, \psi_{1}\right\}$ and $\sin \left(\psi_{2}\right)$ are independent. Here, if $A \equiv a$ and $B \equiv b$, being $a$ equal to $b$ or not, then $A \circ \psi_{0}$ and $B \circ \psi_{1}$ are independent (if that seems unintuitive, some calculations should make it clear). When $a=b$, we have a pair of identically distributed but independent measurable functions.

Even more, as independence is preserved under groupings, if $\left(\psi_{j}:(X, \mathcal{A}) \rightarrow\left(Y_{j}, \mathcal{B}_{j}\right)\right)_{j=0}^{k-1}$ is a family of independent measurable functions and $\dot{\cup}_{i=0}^{q-1} I_{i}=\{0, \ldots, k-1\}, 0 \leq q-1 \leq k-$ 1 , is a partition of $\{0, \ldots, k-1\}$ whose components are non empty, then $\left(\cup_{j \in I_{i}}\left\{\psi_{j}\right\}\right)_{i=0}^{q-1}$ and $\left(\sigma\left(\cup_{j \in I_{i}}\left\{\psi_{j}\right\}\right)\right)_{i=0}^{q-1}$ are also independent, therefore $\int g_{0} \cdot \ldots \cdot g_{q-1} d \mu=\int g_{0} d \mu \cdot \ldots \cdot \int g_{q-1} d \mu, \forall g_{0} \epsilon$ $\mathcal{F}_{\sigma\left(\cup_{j \in I_{0}}\left\{\psi_{j}\right\}\right)}, \ldots, g_{q-1} \in \mathcal{F}_{\sigma\left(\cup_{j \in I_{q-1}}\left\{\psi_{j}\right\}\right)}$, whenever every integral is well defined. But, since $\psi_{j} \epsilon$ $\mathcal{F}_{\sigma\left(\cup_{j \in I_{i}}\left\{\psi_{j}\right\}\right)}, \forall j \in I_{i}$, any measurable function $g_{i}$ on the product space $\left(\prod_{j \in I_{i}} Y_{j}, \otimes_{j \in I_{i}} \mathcal{B}_{j}\right)$ has the property that $g_{i} \circ\left(\psi_{j_{i, 0}}, \ldots, \psi_{j_{i, p_{i}-1}}\right)$, after a composition with another $\overline{\mathbb{R}}$ valued measurable function, is in $\mathcal{F}_{\sigma\left(\cup_{j \in I_{i}}\left\{\psi_{j}\right\}\right)}$, where $I_{i}$ is enumerated as $\left\{0, \ldots, p_{i}-1\right\}$. By proposition 2.3.8, $\left(g_{i} \circ\right.$ $\left.\left(\psi_{j_{i, 0}}, \ldots, \psi_{j_{i, p_{i}-1}}\right)\right)_{i=0}^{q-1}$ is an independent family of measurable functions. That is a generalization of the previous corollary. To illustrate, if $\psi_{0}, \psi_{2}$ and $\psi_{1}$ are independent, then (a) $\psi_{0}+\psi_{1}$ and $\psi_{2}$ are independent and (b) $e^{\psi_{0}}$ and $\max \left\{\psi_{1} \cdot \psi_{2}, \psi_{1}+\psi_{2}\right\}$ are independent.

Our last observation is that independence does not have automatic transitive properties:

(i) $\psi_{0}$ and $\psi_{1}$ independent, $\psi_{1}$ and $\psi_{2}$ independent $\not \psi_{0}$ and $\psi_{2}$ independent: that is easy, just choose to start with $\psi_{0}=\psi_{2}$,

(ii) $\psi_{0}$ and $\psi_{1}$ dependent, $\psi_{1}$ and $\psi_{2}$ dependent $\not \Rightarrow \psi_{0}$ and $\psi_{2}$ dependent: let be $X=\{(0,0),(0,1)$, $(1,0),(1,1)\}, \psi_{0}(x, y)=x, \psi_{1}(x, y)=x y$ and $\psi_{2}(x, y)=y$ (it is intuitive why it is a counterexample, but one should do some calculations to make it clear),

(iii) $\psi_{0}$ and $\psi_{1}$ dependent, $\psi_{1}$ and $\psi_{2}$ independent $\not \psi_{0}$ and $\psi_{2}$ independent: let $X$ be as in (ii), $\psi_{0}(x, y)=x y, \psi_{1}(x, y)=x$ and $\psi_{2}(x, y)=y$.

Next, we comment on the probabilistic interpretation of integration with respect to $\mu$ and some other integrals probabilists use to summarize measurable functions.

Let $\psi$ be a $\overline{\mathbb{R}}$ valued measurable function on $X$. If $\psi$ is in $L^{1}(\mu, \overline{\mathbb{R}})$, its integral $\int \psi d \mu$ is to be interpreted not (only) as an area whose "basis" has a specific mass distribution, but as a value that indicates an average or expected value (we may use this terms as synonyms for the integral), in the sense that the function is integrated and divided by the unitary mass of the domain of integration or in the sense that outcomes are weighted by the associated chances and summed. When $\int \psi d \mu= \pm \infty$, an analogous interpretation holds. A useful characterization is the following.

Proposition 2.3.9 (Expected value integral characterization). Let $\psi$ be a $\mathbb{R}$ measurable function on $(X, \mathcal{A})$. Then, the cumulative distribution function $F$ induced by $\mu_{*} \psi$ is a $[0,1]$-valued measurable function on $\mathbb{R}$ and $\int \psi d \mu=\int_{0}^{\infty} 1-F d m-\int_{-\infty}^{0} F d m=\int_{0}^{\infty} \mu(\{x \in X: \psi(x)>s\}) d m(s)-\int_{-\infty}^{0} \mu(\{x \in$ $X: \psi(x) \leq s\}) d m(s)$ ( $m=$ Lesbegue $)$, whenever one of them is defined $( \pm \infty$ allowed $)$.

One can also try to define, for $1 \leq p<\infty, \int\left[\psi-\left(\int \psi d \mu\right)\right]^{p} d \mu$, named the $\mathrm{p}$-th order centered moment of $\psi$. It will be defined and finite, if, and only if, $\psi$ is in $L^{p}(\mu, \overline{\mathbb{R}})$ (hence $\psi$ is in $L^{1}(\mu, \overline{\mathbb{R}})$, by proposition 2.1.33). The second order centered moment is also called variance, denoted $\operatorname{var}(\psi)$. 
In probability, it represents how much a measurable function is spread around its average value. We will use this variance notation due to is brevity. Note that $\operatorname{var}(\psi)=\int\left[\psi-\left(\int \psi d \mu\right)\right]^{2} d \mu=$ $\int\left[\psi^{2}-2 \psi\left(\int \psi d \mu\right)+\left(\int \psi d \mu\right)^{2}\right] d \mu=\int \psi^{2} d \mu-2\left(\int \psi d \mu\right)\left(\int \psi d \mu\right)+\left(\int \psi d \mu\right)^{2}=\int \psi^{2} d \mu-\left(\int \psi d \mu\right)^{2}$. When $\psi$ is in $L^{1}(\mu, \overline{\mathbb{R}})$ and its $p$-th centered moment is $\pm \infty$, the analogous interpretation holds (we are not interpreting when $\int \psi d \mu= \pm \infty$ and its $p$-th centered moment is $\left.\pm \infty\right)$. When $\psi$ is in $L^{1}(\mu, \overline{\mathbb{R}})$ and $p$ is even, its $p$-th centered moment is always defined in $[0, \infty]$.

The following proposition indicates that integrating a $\overline{\mathbb{R}}$-valued measurable function $g$ defined on the codomain of a measurable function $\psi$ with respect to the measure $\psi_{\star} \mu$ equals to integrating the $\overline{\mathbb{R}}$ valued measurable function $g \circ \psi$ (directly) on the domain of $\psi$ with respect to the measure $\mu$.

Proposition 2.3.10 (Distributions and integrals). Let $(X, \mathcal{A}, \mu)$ be a probability space, $(Y, \mathcal{B})$ a measurable space, $\psi:(X, \mathcal{A}) \rightarrow(Y, \mathcal{B})$ be a measurable function and $g:(Y, \mathcal{B}) \rightarrow \overline{\mathbb{R}}$ be any measurable function.

Then $\int g \circ \psi d \mu=\int g d\left(\psi_{*} \mu\right)$, for all measurable $g$ such that (at least) one of the integrals is well defined $( \pm \infty$ allowed $)$.

In the above notation and conditions, being $\psi$ and $\varphi$ two identically distributed $\overline{\mathbb{R}}$ valued measurable functions, note that, $\int \psi d \mu=\int i d_{\overline{\mathbb{R}}} \circ \psi d \mu=\int i d_{\overline{\mathbb{R}}} d\left(\psi_{*} \mu\right)=\int i d_{\overline{\mathbb{R}}} d\left(\varphi_{*} \mu\right)=\int i d_{\overline{\mathbb{R}}} \circ \varphi d \mu=$ $\int \varphi d \mu$. Let be $k:=\int \psi d \mu=\int \varphi d \mu$ and $g: x \in \overline{\mathbb{R}} \mapsto(x-k)^{p}$, then $\int\left[\psi-\left(\int \psi d \mu\right)\right]^{p} d \mu=\int(\psi-k)^{p} d \mu=$ $\int g \circ \psi d \mu=\int g d\left(\psi_{*} \mu\right)=\int g d\left(\varphi_{*} \mu\right)=\int g \circ \varphi d \mu=\int(\varphi-k)^{p} d \mu=\int\left[\varphi-\left(\int \varphi d \mu\right)\right]^{p} d \mu$. Therefore, if $\psi$ and $\varphi$ are identically distributed and one of them has a well defined integral ( $\pm \infty$ allowed), they will have identical averages, furthermore, if both are in $L^{1}(\mu, \overline{\mathbb{R}})$ and one of them as a well defined the $p$-th centered moment $( \pm \infty$ allowed), they will have identical $p$-th centered moment.

Here the integral still enjoys every property presented in section 2.1. The linearity of the integral functional in proposition 2.1.23 can be enlarged to $\overline{\mathbb{R}}$-valued measurable functions whose integral exists and is in $[-\infty, \infty]$, whenever the sum that pops up is well defined.

With respect to the $p$-th centered moment, things naturally changes slightly. The $p$-th centered moment functional is not homogeneous of degree 1 , but of degree $p: \int\left[a \psi-\left(\int a \psi d \mu\right)\right]^{p} d \mu=\int[a \psi-$ $\left.\left(a \int \psi d \mu\right)\right]^{p} d \mu=\int\left[a\left(\psi-\int \psi d \mu\right)\right]^{p} d \mu=\int a^{p}\left[\psi-\left(\int \psi d \mu\right)\right]^{p} d \mu=a^{p} \int\left[\psi-\left(\int \psi d \mu\right)\right]^{p} d \mu$, whenever $a \in \mathbb{R}, 1 \leq p<\infty$ and $\psi$ is a $\overline{\mathbb{R}}$-valued measurable function whose $p$-th centered moment is defined $( \pm \infty$ allowed $)$.

Also, the $p$-th centered moment functional is not additive and a closed form formula to the sum is cumbersome (even more to general finite sums), but the formula for the 2-nd centered moment for the sum of $\bar{R}$-valued measurable functions in $L^{2}(\mu, \overline{\mathbb{R}})$ is informative:

$$
\begin{gathered}
\operatorname{var}(\psi+\varphi)=\int\left[\psi+\varphi-\left(\int \psi+\varphi d \mu\right)\right]^{2} d \mu=\int\left[\left(\psi-\int \psi d \mu\right)+\left(\varphi-\int \varphi d \mu\right)\right]^{2} d \mu \\
=\int\left[\left(\psi-\int \psi d \mu\right)^{2} d \mu+\int\left(\varphi-\int \varphi d \mu\right)^{2} d \mu+\int 2\left(\psi-\int \psi d \mu\right)\left(\varphi-\int \varphi d \mu\right) d \mu\right. \\
=\operatorname{var}(\psi)+\operatorname{var}(\varphi)+2 \operatorname{covar}(\psi, \varphi)
\end{gathered}
$$

, where $\operatorname{covar}(\psi, \varphi)=\int\left(\psi-\int \psi d \mu\right)\left(\varphi-\int \varphi d \mu\right) d \mu=\int \psi \varphi d \mu-\int \psi d \mu \int \varphi d \mu$ (here we are restricting to $\psi$ and $\varphi$ in $L^{2}(\mu, \overline{\mathbb{R}})$ to avoid $\operatorname{var}(\psi)$ or $\operatorname{var}(\varphi)$ being $\infty$ while covar is $\left.-\infty\right)$. For finite sums the following formula holds:

$$
\begin{aligned}
\operatorname{var}\left(\sum_{j=0}^{k-1} \psi_{j}\right)= & \sum_{(j, i) \in\{0, \ldots, k-1\}^{2}, j=i} \operatorname{covar}\left(\psi_{j}, \psi_{i}\right)+\sum_{(j, i) \in\{0, \ldots, k-1\}^{2}, j \neq i} \operatorname{covar}\left(\psi_{j}, \psi_{i}\right) \\
& =\sum_{j=0}^{k-1} \operatorname{var}\left(\psi_{j}\right)+\sum_{(j, i) \in\{0, \ldots, k-1\}^{2}, j \neq i} \operatorname{covar}\left(\psi_{j}, \psi_{i}\right)
\end{aligned}
$$

The quantity covar $(\psi, \varphi)$, introduced above, is called the covariance between $\psi$ and $\varphi$ and is defined taking values in $\mathbb{R}$ whenever $\psi, \varphi \in L^{2}(\mu, \overline{\mathbb{R}})$, since $\left(\psi-\int \psi d \mu\right)$ and $\left(\varphi-\int \varphi d \mu\right)$ will 
be in $L^{2}(\mu, \overline{\mathbb{R}})$ and, once 2 and 2 are conjugate exponents, their product will be in $L^{1}(\mu, \overline{\mathbb{R}})$, by proposition 2.1.27. Covariance measures if and how much the two measurable functions vary in the same direction with respect to their average while their argument $x$ is varying in $X$. If $\operatorname{covar}(\psi, \varphi)>0, \psi$ and $\varphi$ on average move in the same direction, what magnifies $\operatorname{var}(\psi+\varphi)$; otherwise they on average move in opposite directions, what diminishes $\operatorname{var}(\psi+\varphi)$. Obviously, $\operatorname{covar}(\psi, \varphi)=\operatorname{covar}(\varphi, \psi)$ and when $\psi=\varphi, \operatorname{covar}(\psi, \varphi)=\operatorname{var}(\psi)=\operatorname{var}(\varphi)$.

One can expect that if $\psi, \varphi \in L^{2}(\mu, \overline{\mathbb{R}})$ are independent, $\operatorname{covar}(\psi, \varphi)$ cannot be anything other than zero. That is true, as we know from a corollary of 2.3.8: if $\psi$ and $\varphi$ are independent, then $\int \psi \varphi d \mu=\int \psi d \mu \int \varphi d \mu$, hence $\operatorname{covar}(\psi, \varphi)=0$. But the converse is not true: $X=\{(-1,1),(-1,1)$, $(0,0),(1,-1),(1,1)\} ; \mu(\{p\})=1 / 5, \forall p \in X ; \psi(x, y)=x, \varphi(x, y)=y, \int \psi d \mu=-11 / 5+-11 / 5+01 / 5+$ $11 / 5+11 / 5=0$ and, similarly, $\int \varphi d \mu=0$; also $\int \psi \varphi d \mu=-1 \cdot-11 / 5+-1 \cdot 11 / 5+0 \cdot 01 / 5+1 \cdot-11 / 5+-1 \cdot 11 / 5=0$, hence $\operatorname{covar}(\psi, \varphi)=0$; but $\psi$ and $\varphi$ are not independent since $(\psi \otimes \varphi)_{*} \mu(\{-1\} \times\{-1\})=1 / 5 \neq 4 / 25=$ $2 / 5 \cdot 2 / 5=\psi_{\star} \mu(\{-1\}) \varphi_{*} \mu(\{-1\})$.

It is possible then, under independence, to reduce the formula for the variance of the sum: $\operatorname{var}(\psi+\varphi)=\operatorname{var}(\psi)=\operatorname{var}(\varphi)$ and, inducing, $\operatorname{var}\left(\sum_{j=0}^{k-1} \psi_{j}\right)=\sum_{j=0}^{k-1} \operatorname{var}\left(\psi_{j}\right)$.

The quantity $\operatorname{covar}(\psi, \varphi)$ has the disadvantage that its magnitude can be difficult to interpret. This arises from changes in scale only, since it is already translation invariant, given that it controls for the average. To correct for that, we define, for $\psi, \varphi \in L^{2}(\mu, \overline{\mathbb{R}})$ with non-zero variance, the correlation between $\psi$ and $\varphi, \operatorname{cor}(\psi, \varphi)=\operatorname{covar}\left(\psi_{\text {norm }}, \varphi_{\text {norm }}\right)$, where, for $\lambda=\psi$ or $\varphi, \lambda_{\text {norm }}=$ $\left(\lambda-\int \lambda d \mu\right) / \sqrt{\operatorname{var}(\lambda)}$ is the standardization of $\lambda$, whose variance is 1 and average is 0 (as mentioned, the correction for the average is not really important in this case). The next proposition summarizes a few properties of correlation.

Proposition 2.3.11 (Correlation measures strengh of linear dependence). Let be $\psi, \varphi \in L^{2}(\mu, \overline{\mathbb{R}})$. Then:

(i) $\operatorname{cor}(\psi, \varphi)=\operatorname{cor}(a \psi+b, c \varphi+d), \forall a, c \in(0, \infty), b, d \in \mathbb{R}$

(ii) $-1 \leq \operatorname{cor}(\psi, \varphi) \leq 1$

(iii) $\operatorname{cor}(\psi, \varphi)= \pm 1 \Leftrightarrow \exists b \in \mathbb{R}, a \in(0, \infty)$ (respectively, $a \in(-\infty, 0)$ ) such that $\varphi=a \psi+b$, $\mu$-a.e..

Unlike variance and like independence, covariance and correlation are properties of measurable functions that can not be reduced to their distributions. For such, we use the counterexample given for a similar assertion about independence: there we have $\psi$ and $\varphi$ with $\operatorname{covar}(\psi, \varphi)=0$ and $\psi^{\prime}$ (identically distributed to $\psi$ ) and $\varphi^{\prime}$ (identically distributed to $\varphi$ ) with $\operatorname{covar}(\psi, \varphi)=\operatorname{var}(\psi)>0$; analogously for correlation.

Also, useful inequalities for estimating the probability that a measurable function (or that its distance from the average) is above a given threshold are the following.

Proposition 2.3.12 (Markov's and Chebyshev's inequalities). (i) (Markov's) Let $\psi$ be a $[0, \infty]$ valued measurable function on $(X, \mathcal{A})$ (whose average is always defined but not necessarily finite) and $b>0$, then $\mu(\{x \in X: \psi(x)>b\}) \leq \int \psi d \mu / b$

(ii) (Generalized Markov) Let $\psi$ be a $\mathbb{R}$ valued measurable function on $(X, \mathcal{A}), f: \mathbb{R} \rightarrow[0, \infty)$ be a non-decreasing function and $b>0$, then $\mu(\{x \in X: f \circ \psi(x)>b\}) \leq \int f \circ \psi d \mu / f(b)$

(iii) (Chebyshev's) Let $\psi$ be a measurable function on $(X, \mathcal{A})$ in $L^{1}(\mu, \overline{\mathbb{R}})$ (whose variance is always defined but not necessarily finite) and $b>0$, then $\mu\left(\left\{x \in X:\left|\psi(x)-\int \psi d \mu\right|>b\right\}\right) \leq \operatorname{var}(\psi) / b^{2}$, or equivalently, $\mu\left(\left\{x \in X:\left|\psi(x)-\int \psi d \mu\right|>b \sqrt{\operatorname{var}(\psi)}\right\}\right) \leq 1 / b^{2}$.

We are now able to introduce a couple of useful characterizations of $\mu$-a.e. convergence of $\mathbb{R}$ valued measurable functions.

Proposition 2.3.13 (Equivalence with $\mu$-a.e. convergence). Let $\left(\psi_{n}\right)_{n \geq 0}$ be a sequence of $\mathbb{R}$-valued measurable functions on $(X, \mathcal{A})$ and $\psi$ another one. Then:

(i) $\forall \epsilon>0, \sum_{n} \mathcal{X}_{(\epsilon, \infty)} \circ\left|\psi_{n}-\psi\right|<\infty, \mu$-a.e. $\Leftrightarrow \psi_{n} \rightarrow \psi$, $\mu$-a.e..

(ii) $\lim _{m, n \rightarrow \infty}\left|\psi_{m}-\psi_{n}\right|=0$, $\mu$-a.e. $\Leftrightarrow \psi_{n} \rightarrow \psi$, $\mu$-a.e.. 
Proposition 2.3.14 (Sufficiency for $\mu$-a.e. convergence, v1, Borel-Cantelli Lemmas). Let $\left(A_{n}\right)_{n \geq 0}$ be a sequence of sets in $\mathcal{A}$. Then:

(i) $\sum_{n} \mu\left(A_{n}\right)<\infty \Rightarrow \sum_{n} \mathcal{X}_{A_{n}}<\infty$, $\mu$-a.e.

(ii) $\sum_{n} \mu\left(A_{n}\right)=\infty$ and $\left(A_{n}\right)_{n \geq 0}$ are pairwise independent $\Rightarrow \sum_{n} \mathcal{X}_{A_{n}}=\infty$, $\mu$-a.e.

Proposition 2.3.15 (Sufficiency for $\mu$-a.e. convergence, v2). Let $\left(\psi_{n}\right)_{n \geq 0}$ be a sequence of $\mathbb{R}$-valued measurable functions on $(X, \mathcal{A})$ and $\psi$ another one. Then:

(i) $\forall \epsilon>0, \sum_{n} \mu\left(\left\{x \in X:\left|\psi_{n}(x)-\psi(x)\right|>\epsilon\right\}\right)<\infty \Rightarrow \psi_{n} \rightarrow \psi$, $\mu$-a.e.

(ii) $\exists\left(\epsilon_{n}\right) \searrow 0$ such that $\sum_{n} \mu\left(\left\{x \in X:\left|\psi_{n}(x)-\psi(x)\right|>\epsilon_{n}\right\}\right)<\infty \Rightarrow \psi_{n} \rightarrow \psi$, $\mu$-a.e.

(iii) $\exists\left(\epsilon_{n}\right)$ such that $\sum_{n} \epsilon_{n}<\infty$ and $\sum_{n} \mu\left(\left\{x \in X:\left|\psi_{n+1}(x)-\psi_{n}(x)\right|>\epsilon_{n}\right\}\right)<\infty \Rightarrow \psi_{n} \rightarrow \psi$, $\mu$-a.e.

We now offer a glimpse of concepts of filtrations, tail $\sigma$-algebras and classic $0-1$ laws. Let $(X, \mathcal{A}, \mu)$ be fixed on what is next.

Definition 2.3.16 (Stochastic process). An arbitrary family of measurable functions $\left(\psi_{\alpha}\right)_{\alpha \in \Lambda}$ on $(X, \mathcal{A})$ taking values in the same measurable space $(Y, \mathcal{B})$ is called a stochastic process with state space $(Y, \mathcal{B})$ and parameter set $\Lambda$. Frequently, $\Lambda=\mathbb{N}, \mathbb{Z}$ or $\mathbb{R}$ and the stochastic process is interpreted temporally. As we know, the family $\left(\psi_{\alpha}\right)_{\alpha \in \Lambda}$ can be regarded encapsulated as the joint measurable function $\otimes_{\alpha \in \Lambda} \psi_{\alpha}:(X, \mathcal{A}) \rightarrow\left(\prod_{\alpha \in \Lambda} Y, \otimes_{\alpha \in \Lambda} \mathcal{B}\right)$.

Probabilists interpret a $\sigma$-algebra $\mathcal{F}$ on $X$, subset of $\mathcal{A}$, as a registry of an information state. The motivation comes from the following example: Let be $X=\left\{\left(x_{n}\right)_{n \geq 0}: x_{n} \in E=\{a, b\}\right\}$, whose elements are interpreted as (all-in-one) outcomes of a (repeated) experiment, $\mathcal{A}$ the countable product (of $\mathcal{E}=\mathcal{P}(E)) \sigma$-algebra equipping $X$, and $\left(\psi_{n}=p r_{n}\right)_{n \geq 0}$ a family of measurable functions on $X$ taking values in $E$. After the 3rd partial trial of the experiment, for whatever $x \in X$ to be picked, one will possess only $\operatorname{pr}_{0}(x)=x_{0}, p r_{1}(x)=x_{1}, p r_{2}(x)=x_{2}$, therefore, exactly those functions $\varphi:(X, \mathcal{A}) \rightarrow\left(\overline{\mathbb{R}}, \mathcal{B}_{\overline{\mathbb{R}}}\right)$ that can be expressed as $\varphi=f \circ\left(p r_{0}, p r_{1}, p r_{2}\right)$, for some $f:\left(E^{3}, \otimes_{j=0}^{2} \mathcal{E}\right) \rightarrow$ $\left(\overline{\mathbb{R}}, \mathcal{B}_{\overline{\mathbb{R}}}\right)$, are determined with that partial information. That is, the set of $f$ 's one can plug in determines the body of information available at the end of the $3 \mathrm{rd}$ trial. As always, dealing with measurable $f$ 's, which constitute a set denoted by $M$, we obtain the family $\left(f \circ\left(p r_{0}, p r_{1}, p r_{2}\right)\right)_{f \in M}$ of $\overline{\mathbb{R}}$-valued measurable functions on $X$, which we reduce to the $\sigma$-algebra it generates. But this $\sigma$-algebra coincides with that generated by the measurable function $\left(p r_{0}, p r_{1}, p r_{2}\right)$, which, in turn, coincides with that generated by the family $\left(p r_{j}\right)_{j=0}^{2}$, denoted by $\mathcal{F}$.

In the general case, a $\sigma$-algebra $\mathcal{G}$ on $X$, subset of $\mathcal{A}$, is heuristically understood as the information available to someone who can tell the value of $\varphi(x)$ for all $x \in X$, for all measurable functions $\varphi$ on $(X, \mathcal{G})$ (taking values on $\left(\overline{\mathbb{R}}, \mathcal{B}_{\overline{\mathbb{R}}}\right)$ ).

Definition 2.3.17 (Filtrations). A family $\left(\mathcal{F}_{t}\right)_{t \in T}, T \subset \mathbb{R}$ of $\sigma$-algebras on $X$, contained in $\mathcal{A}$, is called a filtration, whenever $\mathcal{F}_{s} \subset \mathcal{F}_{t}$ for $s \leq t$.

A filtration is to be interpreted as the flow of information gathered from an experiment during a span of time (e.g., $(-\infty, t],[0, t])$, increasing as it progresses. The major proposition regarding filtrations is the following.

Proposition 2.3.18 (Approximations and filtrations). Let $\left(\mathcal{F}_{n}\right)_{n \geq 0}$ be a filtration and $\mathcal{F}_{\infty}=$ $\vee_{n \geq 0} \mathcal{F}_{n}$ be the $\sigma$-algebra generated by $\cup_{n \geq 0} \mathcal{F}_{n}$.

Then for every real-bounded $\overline{\mathbb{R}}$-valued measurable function $\varphi$ on $\left(X, \mathcal{F}_{\infty}\right)$, there exists a sequence $\left(\varphi_{n}\right)_{n \geq 0}$ of real-bounded $\overline{\mathbb{R}}$-valued measurable functions on $\left(X, \mathcal{F}_{n}\right)$ such that (every function is in $L^{1}(\mu, \overline{\mathbb{R}})$ and) $\varphi_{n} \rightarrow \varphi$ in $L^{1}(\mu, \overline{\mathbb{R}})$. Also averages will converge, since $\left|\int \varphi_{n}-\varphi d \mu\right| \leq \int\left|\varphi_{n}-\varphi\right| d \mu \rightarrow 0$.

Let $\left(\mathcal{G}_{n}\right)_{n \geq 0}$ be a sequence of $\sigma$-algebras on $X$, subsets of $\mathcal{A}$, to be understood as the (marginal) information revealed by the $n$-th trial of an experiment. Note that the sequence comprised of $\mathcal{F}_{n}=\bigvee_{j=0}^{n} \mathcal{G}_{j}$ constitutes a filtration, to be understood as the (accumulated) information revealed by until the $n$-th trial of an experiment. We then define the family $\mathcal{H}_{n}=\bigvee_{j>n} \mathcal{G}_{j}, n \geq 0$, each to 
be understood encapsulating the information about the future after $n$. Finally, we define the tail $\sigma$-algebra $\mathcal{H}=\cap_{n} \mathcal{H}_{n}$, the collection of those sets that are in the future for every $n$, also understood as the remote future. We explore some examples to make it clear.

Example 2.3.19. [Tail $\sigma$-algebra] Let $\left(\psi_{n}\right)_{n \geq 0}$ be a sequence of $\overline{\mathbb{R}}$-valued measurable functions on $X,\left(\sum_{j=0}^{n} \psi_{j}\right)_{n \geq 0}$ be the sequence of its partial sums and $\mathcal{G}_{n}=\sigma\left(\left\{\psi_{n}\right\}\right)$ the $\sigma$-algebra induced by $\psi_{n}$. Then:

i) The set $W=\left\{x \in X: \lim _{n} \sum_{j=0}^{n} \psi_{j}(x)\right.$ exists $\}$ is in $\mathcal{H}_{n}, \forall n \geq 0$, hence in $\mathcal{H}$. For such, note that, for any $n \geq 0, \psi_{n+1}, \psi_{n+2}, \ldots$ are measurable on $\left(X, \mathcal{H}_{n}\right)$, as well as $\sum_{j=n+1}^{m} \psi_{j}, \forall m \geq n+1$, $l s_{n}:=\lim \sup _{m} \sum_{j=n+1}^{m} \psi_{j}, l i_{n}:=\liminf _{m} \sum_{j=n+1}^{m} \psi_{j}$ and the difference $d_{n}=l s_{n}-l i_{n}$. But $W=\{x \in$ $\left.X: d_{n}(x)=0\right\}=d_{n}{ }^{-1}(\{0\}), \forall n \geq 0$, hence $W \in \mathcal{H}_{n}, \forall n \geq 0$.

ii) The set $W=\left\{x \in X: \lim \sup _{n} 1 / n \sum_{j=0}^{n-1} \psi_{j}(x)>b\right\}$ is in $\mathcal{H}_{n}, \forall n \geq 0$, hence in $\mathcal{H}$. For such, note that $\limsup _{n} 1 / n \sum_{j=0}^{n-1} \psi_{j}=\lim \sup _{m} 1 / m\left(\psi_{0}+\ldots+\psi_{n}+\sum_{j=n+1}^{m-1} \psi_{j}\right)=\lim _{m} 1 / m\left(\psi_{0}+\ldots+\psi_{n}\right)+$ $\limsup \sup _{m} 1 / m \sum_{j=n+1}^{m-1} \psi_{j}=\lim \sup _{m} 1 / m \sum_{j=n+1}^{m-1} \psi_{j}=: l s_{n}, \forall n \geq 0$, hence $W=\left\{x \in X: l s_{n}(x)>b\right\}=$ $l s_{n}{ }^{-1}((b, \infty)), \forall n \geq 0$, so $W \in \mathcal{H}_{n}, \forall n \geq 0$.

The takeaway is that sets in $\mathcal{H}$ can be defined irrespective to what is in $\psi_{0}, \ldots, \psi_{n}, \forall n \geq 0$. We finish by presenting these last examples:

(iii) $Z=\left\{x \in X: \lim \sup _{n} \sum_{j=0}^{n-1} \psi_{j}(x)>b\right\}$ is not in $\mathcal{H}$

(iv) Let be $B \in \mathcal{B}_{\overline{\mathbb{R}}}$ and $Z=\left\{x \in X: \psi_{n} \in B\right.$ infinitely often $\}$ is in $\mathcal{H}$, for every $n$.

(v) Let be $B \in \mathcal{B}_{\overline{\mathbb{R}}}$ and $Z=\left\{x \in X: \sum_{j=0}^{n-1} \psi_{j}(x) \in B\right.$ infinitely often $\}$ is in not $\mathcal{H}$, for any $n$.

Theorem 2.3.20 (Kolmogorov 0-1 law). If $\left(\mathcal{G}_{n}\right)_{n \geq 0}$ is an independent family of $\sigma$-algebras on $X$, subsets of $\mathcal{A}$, then for every $H \in \mathcal{H}, \mu(H) \in\{0,1\}$.

As a corollary, with the above hypothesis, if $\psi$ is an $\overline{\mathbb{R}}$ (or $\mathbb{R}$ ) valued measurable function on $(X, \mathcal{H})$, then there exists $c \in \overline{\mathbb{R}}$ (or $\mathbb{R}$ ) such that $\psi$ is $\mu$-a.e. equal to $c$ (divide the codomain in compact unit intervals, choosing to recursively subdivide those whose preimages have measure 1 ). In particular, if this hypothesis holds in the previous example, the function $\lim \sup _{n} 1 / n \sum_{j=0}^{n-1} \psi_{j}$ will be $(X, \mathcal{H})$ measurable, since, by (ii) above, $\limsup _{n} 1 / n \sum_{j=0}^{n-1} \psi_{j}{ }^{-1}((b, \infty)) \in \mathcal{H}, \forall b \in \overline{\mathbb{R}}$, hence it is $\mu$-a.e constant.

The next result gives similar conclusions, under tighter hypothesis, using the so called finite permutation invariant sets, which we define next.

Let $\left(\psi_{n}:(X, \mathcal{A}) \rightarrow(Y, \mathcal{B})\right)_{n \geq 0}$ be a family of measurable functions, $\Psi:=\bigotimes_{n \geq 0} \psi_{n}$ its joint function and $\mathcal{F}_{n}=\sigma\left(\left\{\psi_{0}, \ldots, \psi_{n}\right\}\right), n \geq 0$, be the associated filtration. Let $p: \mathbb{N} \rightarrow \mathbb{N}$ be finite permutation, i.e., a bijection such that $p(n)=n$ for all but finitely many $n$ 's. Abusing the notation for composition, we denote by $p \circ \Psi=\left(\psi_{p(0)}, \psi_{p(1)}, \ldots\right)$ the re-arrangement of the entries of $\Psi$ according to $p$. For other $\mathcal{F}_{\infty}$ measurable functions ( $\Psi$ is one such, for the same reason it is $\mathcal{A}$ measurable, its entries are all $\mathcal{F}_{\infty}$, respectively $\mathcal{A}$, measurable), which, by proposition 2.3.2, are of the form $g=h \circ \Psi$, for appropriate $h$, we extend the notation and let be $p \circ g:=h \circ(p \circ \Psi)$.

If $\left(\psi_{n}\right)_{n \geq 0}$ is independent and identically distributed, $\Psi$ and $p \circ \Psi$ will still have the same distribution, for any finite permutation $p$ : let $N$ be such that $p(n)=n, \forall n \geq N$, and let $\prod_{n \geq 0} E_{n}, E_{n} \in \mathcal{B}_{n}, n \geq$ 0 , be a rectangle in set of those rectangles generating $\otimes_{n \geq 0} \mathcal{B}_{n}$. Since independence is preserved under groupings, $\left(\psi_{n}\right)_{n=0}^{N-1}$ and $\left(\psi_{n}\right)_{n \geq N}$ are independent, as well as $\left(\psi_{p(n)}\right)_{n=0}^{N-1}$ and $\left(\psi_{p(n)}\right)_{n \geq N}$, hence $\mu_{*}\left(\bigotimes_{n \geq 0} \psi_{n}\right)=\mu_{*}\left(\bigotimes_{n=0}^{N-1} \psi_{n}\right) \times \mu_{*}\left(\bigotimes_{n \geq N} \psi_{n}\right)$ and $\mu_{*}\left(\bigotimes_{n \geq 0} \psi_{p(n)}\right)=\mu_{*}\left(\bigotimes_{n=0}^{N-1} \psi_{p(n)}\right) \times \mu_{*}\left(\bigotimes_{n \geq N} \psi_{p(n)}\right)$. We want to check that $\mu_{*}\left(\bigotimes_{n \geq 0} \psi_{n}\right)=\mu_{*}\left(\bigotimes_{n \geq 0} \psi_{p(n)}\right)$, but since $\mu_{*}\left(\bigotimes_{n \geq N} \psi_{n}\right)=\mu_{*}\left(\bigotimes_{n \geq N} \psi_{p(n)}\right)$, it suffices to check that $\mu_{*}\left(\bigotimes_{n=0}^{N-1} \psi_{n}\right)=\mu_{*}\left(\bigotimes_{n=0}^{N-1} \psi_{p(n)}\right)$. Note $\mu_{*}\left(\bigotimes_{n=0}^{N-1} \psi_{n}\right)\left(\prod_{n=0}^{N-1} E_{n}\right)=\mu_{*} \psi_{0}\left(E_{0}\right) \ldots$ $\mu_{*} \psi_{N-1}\left(E_{N-1}\right)$ and $\mu_{*}\left(\otimes_{n=0}^{N-1} \psi_{p(n)}\right)\left(\prod_{n=0}^{N-1} E_{n}\right)=\mu_{*} \psi_{p(0)}\left(E_{0}\right) \ldots \mu_{*} \psi_{p(N-1)}\left(E_{N-1}\right)$, which are termwise equal because all $\psi_{n}$ are identically distributed. As a consequence, any $\mathcal{F}_{\infty}$ measurable function $g$ and $p \circ g$ will have the same distribution, for any finite permutation $p$.

We then define a $\mathcal{F}_{\infty}$ measurable function $g$ to be permutation invariant if $p \circ g=g$, for all finite permutation $g$. Also, a set in $\mathcal{F}_{\infty}$ is permutation invariant if its indicator function also is. These are to be understood as functions or sets whose definition is unaffected by finite permutations of the $\psi_{n}$ 's. Examples are all those in example 2.3.19, including (iii) and (v). 
Theorem 2.3.21 (Hewitt-Savage 0-1 law). If $\left(\psi_{n}:(X, \mathcal{A}) \rightarrow(Y, \mathcal{B})\right)_{n \geq 0}$ is an independent and identically distributed family of measurable functions, then the collection of permutation invariant sets constitutes a $\sigma$-algebra, subset of $\mathcal{A}$, denoted $\mathcal{I}$ (which contains $\mathcal{H}$, as induced by $\left(\psi_{n}\right)_{n \geq 0}$ ), and for every $I \in \mathcal{I}, \mu(I) \in\{0,1\}$.

As with Kolmogorov's law, with the above hypothesis, if $\psi$ is an $\overline{\mathbb{R}}$ (or $\mathbb{R}$ ) valued measurable function on $(X, \mathcal{I})$, i.e. a permutation invariant measurable function, then there exists $c \in \overline{\mathbb{R}}$ (or $\mathbb{R}$ ) such that $\psi$ is $\mu$-a.e. equal to $c$.

The following example concludes this topic.

Example 2.3.22 (Random walks). Let $\left(\psi_{n}\right)_{n \geq 0}$ be a sequence of independent and identically distributed $\overline{\mathbb{R}}$ valued measurable functions on $X$ such that $\mu\left(\left\{x \in X: \psi_{0}(x)=0\right\}\right)=a<1$ (to avoid triviality), and $\left(\sum_{j=0}^{n} \psi_{j}\right)_{n \geq 0}$ be the sequence of its partial sums. This last stochastic process is called a random walk on $\overline{\mathbb{R}}$. Then exactly one of the following holds $\mu$-a.e.:

(i) $\lim _{n} \sum_{j=0}^{n} \psi_{j}=\infty$

(ii) $\lim _{n} \sum_{j=0}^{n} \psi_{j}=-\infty$

(iii) $\liminf \operatorname{in}_{n} \sum_{j=0}^{n} \psi_{j}=-\infty$ and $\lim \sup _{n} \sum_{j=0}^{n} \psi_{j}=\infty$

To make sense of it, note that $\lim _{\sup _{n}} \sum_{j=0}^{n} \psi_{j}$ is a permutation invariant measurable function, hence there exists $c \in \overline{\mathbb{R}}$ such that $\limsup _{n} \sum_{j=0}^{n} \psi_{j}$ is $\mu$-a.e. equal to $c$. Similarly, $\limsup _{n} \sum_{j=1}^{n+1} \psi_{j}$ $\mu$-a.e. equal to $d$, but by a direct calculation it is also $\mu$-a.e. equal to $c-\psi_{0}$. Hence $d=c+\psi_{0}$, $\mu$-a.e.. If both $d$ and $c$ were in $\mathbb{R}$, we would have $\psi_{0}=d-c, \mu$-a.e., and (i) if $c=d$, that would imply $\psi_{0}=0, \mu$-a.e., contradicting our hypothesis, (ii) if $d>c$ (without loss of generality), that would imply $\psi_{0}=d-c=e>0, \mu$-a.e., hence $\psi_{n}=e, \mu$-a.e., intersecting this sets we would arrive in a set of full measure where any of the initial limsup's would be identically $\infty$, contradicting $c, d \in \mathbb{R}$. If one them is $\pm \infty$ and another in is $\mathbb{R}$, e.g. $d= \pm \infty, c \in \mathbb{R}$ (without loss of generality), $\psi_{0}= \pm \infty$, leading to the same flaw as in (ii). Hence $c, d \in\{-\infty, \infty\}$, and the only way for " $d=c+\psi_{0}$ " to hold is that $c, d=$ $\pm \infty$. With the same argument we are able to conclude that $\liminf \sum_{j=0}^{n}, \lim \sup _{n} \sum_{j=0}^{n} \in\{-\infty, \infty\}$. Excluding the impossible case when $\liminf _{n} \sum_{j=0}^{n}=\infty$ and $\lim \sup _{n} \sum_{j=0}^{n}=-\infty$, we are left with the cases in the conclusion of the example.

What is left now are the classical law of large numbers results. These theorems are going to be discussed in depth later in section 4 . To continue in this direction, the reader can proceed to section 4.2 .2 .

\subsection{Dynamical Systems and Ergodic Theory}

In this text we will only consider dynamical systems over $\mathbb{N}$ or $\mathbb{R}$, which will be called discrete or continuous, respectively.

In the discrete case a dynamical system is a function $f: X \rightarrow X$ that generates a family of functions $\left(f^{n}: X \rightarrow X\right)_{n \in \mathbb{N}}$, where $f^{0}:=i d, f^{1}:=f, f^{2}:=f \circ f, \ldots, f^{n}:=f \circ f^{n-1}=f^{n-1} \circ f, \ldots$ If $f$ is invertible we could consider an enlarged family $\left(f^{n}: X \rightarrow X\right)_{n \in \mathbb{Z}}$, where $f^{-1}:=f^{-1}$, the inverse, $f^{-2}:=f^{-1} \circ f^{-1}, \ldots, f^{-n}:=f^{-1} \circ f^{-(n-1)}=f^{-(n-1)} \circ f^{-1}, \ldots$ In any case, this family, by definition, satisfies the group property $f^{n+m}=f^{n} \circ f^{m}, \forall m, n$. In particular, if $f$ is invertible, $i d=f^{0}=f^{n-n}=f^{n} \circ f^{-n}$ and $i d=f^{0}=f^{-n+n}=f^{-n} \circ f^{n}$, hence $f^{n}$ is invertible and $f^{-n}$ is its inverse, $\forall n \in \mathbb{Z}$. We could define a discrete dynamical system to be the generated family itself, but it does not make any difference.

In the continuous case a dynamical system is itself a family of functions, called flow, $\left(f^{t}: X \rightarrow\right.$ $X)_{t \in \mathbb{R}}$ that satisfies the group property, i.e., $f^{0}=i d$ and $f^{s+t}=f^{s} \circ f^{t}, \forall s, t \in \mathbb{R}$. In particular, $i d=f^{0}=f^{t-t}=f^{t} \circ f^{-t}$ and $i d=f^{0}=f^{-t+t}=f^{-t} \circ f^{t}$, hence $f^{t}$ is invertible and $f^{-t}$ is its inverse, $\forall t \in \mathbb{R}$. There is no analogous to a generating function here.

Continuous dynamical systems are generally obtained from ordinary differential equations of the form $\gamma^{\prime}(t)=F(\gamma(t))$, with $\gamma(0)=x$. The set $X$ is a space where a notion of derivative of $X$-valued functions on $\mathbb{R}$ can be defined, $x$ is some point in $X, F: X \rightarrow X$ is a vector field and one wants to 
find a differentiable $\gamma: \mathbb{R} \rightarrow X$ such that itself and $\gamma^{\prime}: \mathbb{R} \rightarrow X$ make the first equation hold, while satisfying $\gamma(0)=x$. We denote such solution $\gamma_{x}(t)$. Under adequate conditions for $F$, it can be guaranteed that these solutions exists uniquely and globally (defined in $\mathbb{R}$ ), for every $x \in X$. In this case, using $t$ as parameter and defining $f^{t}: x \in X \mapsto \gamma_{x}(t) \in X$, we obtain a family $\left(f^{t}: X \rightarrow X\right)_{t \in \mathbb{R}}$ that constitutes a flow.

In either cases, discrete or continuous, considering only the non-negative indexed portions of the (ordered) families of functions introduced above and evaluating them at $x$, gives us the so called forward $f$-orbit of $x$. If we consider only the non-positive indexed portions of them (when available), this gives us the so called backward $f$-orbit of $x$. If we consider everything (available), this gives us the $f$-orbit of $x$. We will often have a function $\psi: X \rightarrow \mathbb{R}$, called an observable. Composing $\psi$ with the (forward, backward) $f$-orbit of $x$ gives us the so called $\psi$-observed (forward, backward) $f$-orbit of $x$.

When $X$ is equipped as a measurable space $(X, \mathcal{A})$, we will assume our dynamical system is measurable, i.e., $f$ and $f_{t}$ (for all $t$ ) are measurable in the discrete and continuous cases, respectively. We will also assume any observable $\psi$ is measurable.

When $X$ is equipped as a measure space $(X, \mathcal{A}, \mu)$, we can check how dynamics and measure are related, i.e., whether the former preserves the later.

In the discrete case, with $f$ a measurable function, we say $f$ preserves $\mu$, or $\mu$ is $f$-invariant, or $(f, \mu)$ is an invariant system when $\mu\left(f^{-1}(A)\right)=\mu(A), \forall A \in \mathcal{A}$, where $f^{-1}(A)$ is the preimage of $A$ through $f$. Written in another manner, $(f, \mu)$ is an invariant system if $\mu \circ f^{-1}=\mu$ or $f_{\star} \mu=\mu$. Intuitively, the mass of a set $A$ is equal to the mass of the set of points arriving in $A$ through $f$, or the probability that $f$ takes values in $A$ equals the probability of $A$ itself, or more specifically, the probability that a point $x$ is in $A$ equals the probability that the $f$-image of $x^{\prime}$ is in $A$.

To avoid confusions, here are some useful facts about preimages. Let $f: X \rightarrow Y$ (not necessarily injective, surjective or bijective), then:

(o) If $f$ is a bijection, i.e., invertible, then $f^{-1}(A)=\{x \in X: f(x) \in A\}$ and $f^{-1}(A)=\left\{f^{-1}(x)\right.$ : $x \in A\}$ are identical sets.

(i) In general, $A \subset f^{-1}(f(A)), \forall A \subset X$. But $f$ is injective $\Leftrightarrow A=f^{-1}(f(A)), \forall A \subset X$.

(ii) In general, $f\left(f^{-1}(B)\right) \subset B, \forall B \subset X$. But $f$ is surjective $\Leftrightarrow f\left(f^{-1}(B)\right)=B, \forall B \subset X$.

(iii) By (i) and (ii), $f$ is bijective $\Leftrightarrow f\left(f^{-1}(C)\right)=C=f^{-1}(f(C)), \forall C \subset X$.

(iv) Preimages and images preserve inclusion: $A \subset B \Rightarrow f^{-1}(A) \subset f^{-1}(B), f(A) \subset f(B)$.

(v) Preimages and images preserve arbitrary union: $f^{-1}\left(\cup_{\alpha \in \Lambda} A_{\alpha}\right)=\cup_{\alpha \in \Lambda} f^{-1}\left(A_{\alpha}\right), f\left(\cup_{\alpha \in \Lambda} A_{\alpha}\right)=$ $\cup_{\alpha \in \Lambda} f\left(A_{\alpha}\right)$.

(vi) Preimages preserve arbitrary intersection but images do not necessarily do so: $f^{-1}\left(\cap_{\alpha \in \Lambda} A_{\alpha}\right)=$ $\cap_{\alpha \in \Lambda} f^{-1}\left(A_{\alpha}\right), f\left(\cap_{\alpha \in \Lambda} A_{\alpha}\right) \subset \cap_{\alpha \in \Lambda} f\left(A_{\alpha}\right)$.

(vii) Preimages preserve differences (and complements) but images do not necessarily do so: $f^{-1}(A) \backslash f^{-1}(B)=f^{-1}(A \backslash B), f(A) \backslash f(B) \subset f(A \backslash B)$

(viii) Preimages and images behave well with composition: let $X \stackrel{f}{\rightarrow} Y \stackrel{g}{\rightarrow} Z$, then $(g \circ f)^{-1}(A)=$ $f^{-1}\left(g^{-1}(A)\right)$ and $(g \circ f)(A)=g(f(A))$.

When a measurable dynamical system $f$ on $(X, \mathcal{A}, \mu)$ is invertible, we are mostly concerned if $f^{-1}$ is also measurable (this does not always happen), i.e., when $f$ is a sort of measurable analogous to an homeomorphism, let's say measurable isomorphism. In this case, if $(f, \mu)$ is an invariant system, $\left(f^{-1}, \mu\right)$ will also be, i.e., $f$ also "preserves $\mu$ forwardly": $\mu(A)=\mu\left(f^{-1}(f(A))\right)=\mu(f(A))$.

Iterating the invariance property $(f, \mu)$, it follows that $\left(f^{n}, \mu\right)$ is an invariant system for every $n \in \mathbb{N}$, or, if $f$ is a measurable isomorphism, for all $n \in \mathbb{Z}$.

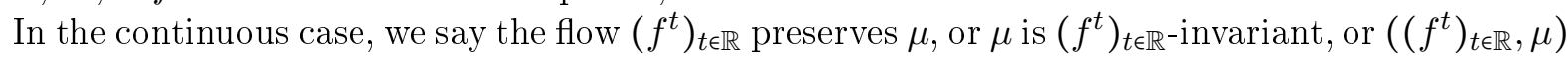
is an invariant system if an analogous to the last paragraph holds directly: $\left(f^{t}, \mu\right)$ is an invariant system for every $t \in \mathbb{R}$, i.e., $\mu\left(f^{-t}(A)\right)=\mu(A), \forall A \in \mathcal{A}, t \in \mathbb{R}$.

Discrete and continuous dynamical systems interplay with each other through many working routines such as lifts, Poincare's sections etc. But that is not of concern here. Most of the concepts to be studied in this section adapt easily to continuous dynamical systems, so to be economic we will focus only in the discrete case from now on. Also, from now on, $X$ (a general set, whose structure 
we specify case by case) will always be equipped as measure space $(X, \mathcal{A}, \mu)$ (we are not fixing $\mu$, sometimes we may want to vary it, but for all purposes there should be always some measure equipping the fixed measurable space $(X, \mathcal{A})$ ) and the dynamical system $f: X \rightarrow X$ is at least measurable.

The following characterization of invariant systems is a useful one.

Proposition 2.4.1 (Invariance and integration). Let $(X, \mathcal{A})$ be a measurable space, $f: X \rightarrow X$ be a measurable function and $\mu$ be a measure on $(X, \mathcal{A})$. Then:

(i) $(f, \mu)$ is an invariant system if, and only if, $\int \psi \circ f d \mu=\int \psi d\left(f_{*} \mu\right)=\int \psi d \mu, \forall \psi: X \rightarrow \mathbb{R}$ in $L^{1}(\mu, \mathbb{R})$.

(ii) if $\mu$ is a finite measure, then $\int \psi \circ f d \mu=\int \psi d\left(f_{*} \mu\right)$, for all bounded measurable $\psi: X \rightarrow \mathbb{R}$.

Proof. For (i: $\Rightarrow$ ) and (ii: $\Rightarrow$ ), first pointwise approximate $\psi^{+}$by an increasing sequence of simple functions $\left(\psi_{n}^{+}=\sum_{j=0}^{k_{n}} a_{j} \mathcal{X}_{A_{j}}\right)_{n \geq 0}$. Second, note that $(\psi \circ f)^{+}=\psi^{+} \circ f$ is itself approximated by the increasing sequence of simple functions $\left(\psi_{n}^{+} \circ f=\sum_{j=0}^{k_{n}} a_{j} \mathcal{X}_{A_{j}} \circ f=\sum_{j=0}^{k_{n}} a_{j} \mathcal{X}_{f^{-1}\left(A_{j}\right)}\right)_{n \geq 0}$.

But $\int \psi_{n}^{+} d\left(f_{\star} \mu\right)=\sum_{j=0}^{k_{n}} a_{j} f_{\star} \mu\left(A_{j}\right)=\sum_{j=0}^{k_{n}} a_{j} \mu\left(f^{-1}\left(A_{j}\right)\right)=\int \psi_{n}^{+} \circ f d \mu$ and, by the monotone convergence theorem, we have that $\int \psi_{n}^{+} d f_{*} \mu \rightarrow \int \psi^{+} d f_{*} \mu$ and $\int \psi_{n}^{+} \circ f d \mu \rightarrow \int \psi^{+} \circ f d \mu$. Therefore $\int \psi^{+} d f_{\star} \mu=\int \psi^{+} \circ f d \mu$ and, analogously, $\int \psi^{-} d f_{\star} \mu=\int \psi^{-} \circ f d \mu$.

Since in (i) $\psi$ is $\mu=f_{*} \mu$ integrable, and in (ii) $\psi$ is $f_{*} \mu$-integrable $\left(\psi\right.$ is bounded and $f_{*} \mu$ is a probability), taking the difference between (the left hand side of) the last two equalities is a well defined operation that leads to $\int \psi d f_{*} \mu=\int \psi \circ f d \mu$.

For (i: $\Leftarrow$ ), just choose $\psi$ to be a characteristic function.

Remark. If $X$ is a metric space $M$, a better version of (i: $\Leftarrow),(\mathrm{i}: \Leftarrow)$ ', can be guaranteed with the weakened hypothesis that the integral equality holds for every bounded continuous $\psi$, instead of for every bounded measurable $\psi$. The proof of $(\mathrm{i}: \Leftarrow)$ ' is different from that of $(\mathrm{i}: \Leftarrow)$ ), since characteristic functions are no more, from hypothesis, verifying that integral equality.

Let $X$ be a $\mathrm{LCH}$ topological space, $\left(X, \mathcal{B}_{X}\right)$ be a (Borelian) measurable space, $f: X \rightarrow X$ a measurable function and $\mu$ a measure on $\left(X, \mathcal{B}_{X}\right)$.

Now consider $f_{\star}: \mathcal{M}_{r}(X, \mathbb{R}) \rightarrow \mathcal{M}_{r}(X, \mathbb{R})$ mapping $\mu \mapsto f_{\star} \mu$. Although the pushforward of a measure is another measure, $f_{\star}$ is not well defined because, since $\mu$ being Radon does not imply $f_{*} \mu$ to be Radon. Not even the restriction to $\mathcal{M}_{1, r}(X)$ is well defined, because the regularity properties of the pushforward measure are not straightforward.

So, for now, we must consider $X=M$ to be, additionally, a metric space. In a metric space, every Borelian probability (or finite) measure is regular.

Therefore even though $f_{*}: \mathcal{M}_{r}(M, \mathbb{R}) \rightarrow \mathcal{M}_{r}(M, \mathbb{R})$ is still not well defined, its restriction to $\mathcal{M}_{1, r}(M)$ is properly defined, as the pushforward of a Radon probability will still be a Radon probability. Under theses circumstances, $\mathcal{M}_{1, r}(M)=\mathcal{M}_{1}(M)$ and $f_{\star}: \mathcal{M}_{1}(M) \rightarrow \mathcal{M}_{1}(M)$ can be understood as a dynamical system itself.

Adding to the setting that $(f, \mu)$ is an invariant system, we can define Koopman's operator $U_{f}: L^{p}(\mu, \mathbb{R}) \rightarrow L^{p}(\mu, \mathbb{R}), p \in[1, \infty]$, given by $U_{f}(\psi)=\psi \circ f$.

Koopman's operator is well defined and an isometry because $\left\|U_{f}(\psi)\right\|_{p}=\left(\int\left|U_{f}(\psi)\right|^{p} d \mu\right)^{1 / p}=$ $\left(\int|\psi \circ f|^{p} d \mu\right)^{1 / p}=\left(\int|\psi|^{p} \circ f d \mu\right)^{1 / p}=\left(\int|\psi|^{p} d \mu\right)^{1 / p}=\|\psi\|_{p}$. It is a positive operator, i.e., $\psi \geq 0 \mu$-a.e. $\Rightarrow U_{f}(\psi) \geq 0 \mu$-a.e., since $(\psi \circ f)^{-1}((-\infty, 0))=f^{-1}\left(\psi^{-1}((-\infty, 0))\right)$ whose measure equals that of $\psi^{-1}((-\infty, 0))$, which is zero. Also, it is linear, since $(\psi+\varphi) \circ f=\psi \circ f+\varphi \circ f$. Finally, as an isometry, it is injective, and, if $f$ is invertible, Koopman's operator will also be, with $\left(U_{f}\right)^{-1}=U_{f^{-1}}$, even more, $U_{f}$ will be an isomorphism, once itself and its inverse will be linear bijections on the vector space $L^{p}$.

Assuming further that $f$ is continuous, we would like to adapt the definition of Koopman's operator to get a dynamical system on one of those sets of functions which are related to the weak* topology on $\mathcal{M}_{1, r}(M)$, most directly $C_{c}(X, \mathbb{R})$ or $C_{0}(X, \mathbb{R})$. In any such case $U_{f}$ would be defined independently of $\mu$ (i.e., the assumption that we start with a invariant system is unnecessary), but 
there is nothing guaranteeing that a $\psi$ in one such set that would remain there after left composition with $f$ (e.g., if $f$ is constant).

Switching to $X=\bar{M}$ a compact metric space solves this problem. In fact, the set of $\mathbb{R}$ valued continuous functions on $\bar{M}, C(\bar{M}, \mathbb{R})$, equals $C_{b}(\bar{M}, \mathbb{R}), C_{0}(\bar{M}, \mathbb{R})$ and $C_{c}(\bar{M}, \mathbb{R})$, while $\psi \in C(\bar{M}, \mathbb{R}) \Rightarrow \psi \circ f \in C(\bar{M}, \mathbb{R})$.

Therefore $U_{f}: C(\bar{M}, \mathbb{R}) \rightarrow C(\bar{M}, \mathbb{R})$ is well defined. This function is intimately related to $f_{\star}$ : $\mathcal{M}_{r}(\bar{M}, \mathbb{R}) \rightarrow \mathcal{M}_{r}(\bar{M}, \mathbb{R})$, which is now well defined, since compactness guarantees every measure in $\mathcal{M}_{r}(\bar{M}, \mathbb{R})$ is finite, hence Radon (once $\bar{M}$ is a metric space). The dual of $U_{f}$, which we define next, is $f_{\star}$.

If $X$ and $\mathcal{Y}$ are Banach spaces and $F: X \rightarrow \mathcal{Y}$ is a linear transformation between them, define $F^{*}: T \in \mathcal{Y}^{*} \mapsto T \circ F \in X^{*}$, the so called dual of $F$. When $X=\mathcal{Y}=C(\bar{M}, \mathbb{R}), F=U_{f}: C(\bar{M}, \mathbb{R}) \rightarrow$ $C(\bar{M}, \mathbb{R})$, we obtain $U_{f}^{*}: C(\bar{M}, \mathbb{R})^{*} \rightarrow C(\bar{M}, \mathbb{R})^{*}$ mapping $T \mapsto U_{f}^{*}(T)=T \circ U_{f} \in C(\bar{M}, \mathbb{R})^{*}$, the functional that evaluated at $\psi$ gives $T \circ U_{f}(\psi)=T(\psi \circ f)$. If we rewrite $T$ as $T_{\eta}$, for some $\eta \in \mathcal{M}_{r}(\bar{M}, \mathbb{R})$, it follows that $T_{\eta} \stackrel{U_{f}^{*}}{\mapsto} T_{\eta} \circ U_{f}=T_{f_{*} \eta}$ since $T_{\eta} \circ U_{f}(\psi)=T_{\eta}(\psi \circ f)=\int \psi \circ f d \eta \stackrel{*}{=}$ $\int \psi d\left(f_{*} \eta\right)=T_{f_{*} \eta}(\psi)$. Equality $(*)$ holds by proposition 2.4.1(ii), since $\psi \in C(\bar{M}, \mathbb{R})$ is continuous on a compact set $\bar{M}$, hence bounded, and $\eta \in \mathcal{M}_{r}(\bar{M})$ is Radon on a compact set $\bar{M}$, hence finite. Therefore $U_{f}^{*}$ and $f_{*}$, in this set up, are the same, up to isomorphism.

Summing up, if we start with $X$ LCH and a measurable dynamical system $f: X \rightarrow X$, we should assume further that $X=M$ is also a metric space, in order to derive an associated dynamical system $f_{*}: \mathcal{M}_{1, r}(M) \rightarrow \mathcal{M}_{1, r}(M)$. Also, if $f$ is continuous and $X=\bar{M}$ is a compact metric space, the complete dynamical system $f_{\star}: \mathcal{M}_{r}(X, \mathbb{C}) \rightarrow \mathcal{M}_{r}(X, \mathbb{C})$ is well defined, as well $U_{f}$ : $C(\bar{M}, \mathbb{C}) \rightarrow C(\bar{M}, \mathbb{C})$, with the property that $U_{f}^{*} \equiv f_{\star}$. Additionally, again with $X$ LCH and $f$ measurable, if $(f, \mu)$ is an invariant dynamical system, we can derive the associated dynamical system $U_{f}: L^{p}(\mu, \mathbb{R}) \rightarrow L^{p}(\mu, \mathbb{R}), p \in[1, \infty]$.

Proposition 2.4.2 (Continuity of the push forward). Let $X=\bar{M}$ be a compact metric space, $f: \bar{M} \rightarrow \bar{M}$ a continuous dynamical system and $f_{\star}: \mathcal{M}_{1}(\bar{M}) \rightarrow \mathcal{M}_{1}(\bar{M})$ the associated dynamical introduced above. Then $f_{*}$ is weak*-weak* continuous.

Remark. The function $f_{\star}$ is already well defined for $X$ a LCH metric space, but the weak* topology, as we defined, is for $X=\bar{M}$ a compact metric space.

Proof. For such, take a neighborhood of a point on the image $V_{f_{*} \mu, \mathbf{g}, \epsilon}=\cap_{g_{j} \in \mathbf{g}} U_{f_{*} \mu, g_{j}, \epsilon}, \mathbf{g}=$ $\left\{g_{0}, \ldots, g_{q}\right\} \subset C_{b}(\bar{M}, \mathbb{R})$. Note that $\left|\int g_{j} d\left(f_{\star} \mu\right)-\int g_{j} d\left(f_{\star} \nu\right)\right|=\left|\int g_{j} \circ f d \mu-\int g_{j} \circ f d \nu\right|$, due to proposition 2.4.1 and the fact that $g_{j} \in C_{b}(\bar{M}, \mathbb{R})$. So $\nu \in V_{\mu, \mathbf{g} \circ f, \epsilon} \Rightarrow f_{*} \nu \in V_{f_{*} \mu, \mathbf{g}, \epsilon}$, i.e., $f_{*}\left(V_{\mu, \mathbf{g} \circ f, \epsilon}\right) \subset V_{f_{*} \mu, \mathbf{g}, \epsilon}$, where $\mathbf{g} \circ f=\left\{g_{0} \circ f, \ldots, g_{q} \circ f\right\}$ is still a subset of $C_{b}(\bar{M})$ since $f$ is continuous. That is, we found a neighborhood of $\mu$ whose image is inside the given neighborhood of $f_{\star} \mu$.

Now, still with $X=\bar{M}$ a compact metric space choose any Dirac measure $\delta_{x}(A)=1$ if $x \in A$ and 0 otherwise, for some $x \in X$. Then define $\mu_{n}=1 / n \sum_{j=0}^{n-1} f_{*}^{j} \delta_{x}$, where $f_{*}^{j}=\left(f_{\star}\right)^{j}=\left(f^{j}\right)_{\star}$. Since, by proposition 2.2.3, $\left(\mathcal{M}_{1}(\bar{M})\right.$, weak*) is compact, there exists $\mu \in \mathcal{M}_{1}(\bar{M})$ and a subsequence $\left(n_{k}\right)_{k \geq 0}$ such that $\mu_{n_{k}}=1 / n_{k} \sum_{j=0}^{n_{k}-1} f_{*}^{j} \delta_{x} \stackrel{w *}{\longrightarrow} \mu$ as $k \rightarrow \infty$.

So, for a given $\epsilon>0$ and a set $\mathbf{g}=\left\{g_{0}, \ldots, g_{q}\right\} \subset C_{b}(X, \mathbb{R})$ we have $\mid \int g_{i} d\left(1 / n_{k} \sum_{j=0}^{n_{k}-1} f_{*}^{j} \delta_{x}\right)-$ $\int g_{i} d \mu|=| 1 / n_{k} \sum_{j=0}^{n_{k}-1} \int g_{i} d\left(f_{*}^{j} \delta_{x}\right)-\int g_{i} d \mu|=| 1 / n_{k} \sum_{j=0}^{n_{k}-1} \int g_{i} \circ f^{j} d \delta_{x}-\int g_{i} d \mu \mid<\epsilon / 2, \forall i=0, \ldots, q$, whenever $k \geq n_{0}$. But the pushfoward of this limit measure, $f_{\star} \mu=f_{\star}\left(\lim _{k} 1 / n_{k} \sum_{j=0}^{n_{k}-1} f_{*}^{j} \delta_{x}\right)=$ $\lim _{k} 1 / n_{k} \sum_{j=1}^{n_{k}} f_{*}^{j} \delta_{x}$, has analogous properties, since $\left|1 / n_{k} \sum_{j=0}^{n_{k}-1} \int g_{i} \circ f^{j} d \delta_{x}-1 / n_{k} \sum_{j=1}^{n_{k}} \int g_{i} \circ f^{j} d \delta_{x}\right|=$ $\left|1 / n_{k}\left(\int g_{i} d \delta_{x}-\int g_{i} \circ f^{n_{k}} d \delta_{x}\right)\right| \leq\left|1 / n_{k} \int g_{i} d \delta_{x}\right|+\left|1 / n_{k} \int g_{i} \circ f^{n_{k}} d \delta_{x}\right| \leq 2 / n_{k}\left\|g_{i}\right\|_{u} \leq 2 / n_{k} \max _{i}\left\|g_{i}\right\|_{u}<\epsilon / 2$ whenever $n \geq n_{1} \geq n_{0}$. By the triangular inequality $\left|1 / n_{k} \sum_{j=1}^{n_{k}} \int g_{i} \circ f^{j} d \delta_{x}-\int g_{i} d \mu\right|<\epsilon, \forall i=0, \ldots, q$, whenever $k \geq n_{1}$, hence $1 / n_{k} \sum_{j=1}^{n_{k}} f_{*}^{j} \delta_{x} \stackrel{w *}{\longrightarrow} \mu$. On the other hand we know $\lim _{k} 1 / n_{k} \sum_{j=1}^{n_{k}} f_{*}^{j} \delta_{x}=f_{*} \mu$, so by uniqueness $f_{\star} \mu=\mu$.

What we concluded is the following theorem: 
Theorem 2.4.3 (Existence of invariant measures). Let $\bar{M}$ be a compact metric space and $f: \bar{M} \rightarrow$ $\bar{M}$ be a continuous dynamical system. Then there exists (at least) a $\mu \in \mathcal{M}_{1}(\bar{M})$ such that $(f, \mu)$ is an invariant system, i.e., $f_{*} \mu=\mu$.

Now we know invariant measures really exist in such generality, it is time to understand why they are worth studying.

For the purpose of the current dissertation, we are aiming to discuss one of the contexts where they play a major role, Birkhoff's ergodic theorem.

So what we explain next, recurrence theorems, are just a simple motivation to Birkhoff's ergodic theorem.

Theorem 2.4.4 (Poincare's recurrence theorem v1). Let $(X, \mathcal{A})$ be a measurable space $(X$ is arbitrary) and $f: X \rightarrow X$ a measurable dynamical system. Let $\mu$ be any finite measure on $(X, \mathcal{A})$ such that $(f, \mu)$ is a invariant system.

Then, for every $A \in \mathcal{A}$ such that $\mu(A)>0$, we have that $\mu$-a.e. $x \in A$ recurs to $A$ through $f$, i.e., there exists a natural subsquence $\left(n_{k}\right)_{k \geq 0}$ going to infinity, so that $f^{n_{k}}(x) \in A$.

Proof. Let $B \subset A$ be the set of points not returning to $A$ (not even once). Note that $f^{-j}(B) \cap$ $f^{-k}(B) \neq 0$ for all $j \neq k$. Otherwise, there exists $j<k, k=j+d, d \in \mathbb{N}_{*}$, and $x \in f^{-j}(B) \cap f^{-k}(B)$, and we would have $y=f^{j}(x) \in B$ and $f^{k}(x)=f^{j+d}(x)=f^{d}(y) \in B \subset A$, so $y$ would recur to $A$, a contradiction. Hence $\mu(B)=0$, otherwise a reunion of a large enough quantity of its disjoint preimages would extrapolate the measure of the space.

Therefore, we obtain $G_{1}=A \backslash B \subset A, \mu\left(G_{1}\right)=\mu(A)$, such that $x \in G_{1}$ returns to $A$ at least once, at time $k(x)$, i.e., $f^{k(x)}(x) \in A$. Repeating the argument with $G_{1}$, we obtain $G_{2} \subset G_{1} \subset A, \mu\left(G_{2}\right)=$ $\mu\left(G_{1}\right)=\mu(A)$, such that $x \in G_{2}$ returns to $G_{1}$, at time $k^{\prime}(x)$, i.e., $f^{k^{\prime}(x)}(x) \in G_{1}$. Combining, $x \in G_{2} \Rightarrow f^{k^{\prime}(x)}(x) \in G_{1} \subset A \Rightarrow f^{k(x)+k^{\prime}(x)}(x) \in A$, i.e., $x \in G_{1}$ returns to $A$ at least twice. Finally, every point in $G=\cap_{n \geq 1} G_{n} \subset A$ recurs to $A$ and $\mu(G)=\mu(A)$.

When we admit $X$ is equipped with an adequate topological structure, the theorem gets the following form.

Theorem 2.4.5 (Poincare's recurrence theorem v2). Let $(X, \tau)$ be a topological space admitting a countable basis (i.e., there exists a countable family of open sets such that every open set can be written as a countable union of those), $\left(X, \mathcal{B}_{X}\right)$ be the Borelian measurable space and $f: X \rightarrow X a$ measurable dynamical system. Let $\mu$ be any finite measure on $\left(X, \mathcal{B}_{X}\right)$ such that $(f, \mu)$ is a invariant system.

Then, $\mu$-a.e. $x \in X$ is recurrent through $f$, i.e., there exists a natural subsquence $\left(n_{k}\right)_{k \geq 0}$ going to infinity, so that $f^{n_{k}}(x) \stackrel{k}{\rightarrow} x$.

Proof. Let $U_{j}, j \geq 1$ be an element of the countable basis. Let $\tilde{U}_{j} \subset U_{j}$ be the subset of points not recurring to $U_{j}$. Let $\tilde{U}=\cup_{j \geq 1} \tilde{U}_{j}$. We know $\mu(\tilde{U})=0$, because each has zero measure. Take $G=X \backslash \tilde{U}$ and $x \in G$. For whatever open $U$ with $x \in U$, we see that $x$ returns to $U$, therefore $x$ is topologically recurrent. This is because there is some $U_{j_{0}} \subset U$ and once $x \notin \tilde{U}_{j_{0}}$, we know it recurs to $U_{j_{0}}$ and this suffices.

Remark. In theorem 2.4.4 we said that " $x$ recurs to $A$ " (through $f$ ), a concept that involves a point $x(\in A)$ and a measurable set $A$ - and, of course, a dynamical system $f$ on a measurable space. This is a measure theoretical concept that needs no topology.

In the theorem above we said that " $x$ is recurrent" (through $f$ ), a concept that involves only a point $x$ - and a dynamical system $f$ on a topological space. This is a topological concept that needs no measure theory. It will be addressed again in section 2.5.

Whenever we want to characterize a point in the previous terms, we will be strict in using the words quoted above. If we want to refer to the recurrence concept, in general, of the last paragraphs, we will use, respectively "measure recurrence" and "topological recurrence". If we want to refer to the 
more general concept of either measure recurrence or topological recurrence, we will say "recurrence" only.

The concept of topological recurrence is a generalization of periodicity, in the sense that, $x$ does not return exactly to $x$ after a finite number of iterations of $f$ (and loops with a fixed time period), but returns arbitrarily and repeatedly closer as time progresses (although we are not controlling for the time period of these approximations). Also, a recurrent point $x$ recurs to $A$ whenever $A$ is one of its neighborhoods.

The following theorem is a pure topological counterpart of the above theorems, dealing with topological recurrence but without mention to (invariant) measures.

Theorem 2.4.6 (Birkhoff's recurrence theorem). Let $\bar{M}$ be a compact metric space and $f: \bar{M} \rightarrow \bar{M}$ be a continuous dynamical system. Then there exist a recurrent point through $f$.

Proof. Let $\mathcal{I}$ be the set of non-empty closed sets $K$ in $\bar{M}$ (hence compact sets) such that $f(K) \subset K$. Obviously, $M \in \mathcal{I}$. On $\mathcal{I}$ define the partial order $K_{1} \leq K_{2} \Leftrightarrow K_{1} \subset K_{2}$. Note that any partiallyordered family in $\mathcal{I}$ admits a lower bound in $\mathcal{I}$, the intersection of its constituents ((i) it is non-empty, as the intersection of nested compact sets, (ii) it is closed, as the arbitrary intersection of closed sets and (iii) the image of any point $x$ in the intersection is still in the intersection, since $x$ is in all of the constituents of the family as well as $f(x)$, because each of them is invariant). Using Zorn's lemma, we have that $\mathcal{I}$ has minimal elements, i.e., minimal sets.

Now note that a set $W$, closed and such that $f(W) \subset W$, is minimal with respect to the above partial order if, and only if, $\forall x \in W$, the orbit of $x$ is dense in $W$. The necessity is because, being $W$ closed, and such that $f(W) \subset W$, already means that $W$ contains the orbit of any $x \in W$ $(f(x) \in W \Rightarrow f(f(x)) \in W \ldots)$ as well as its closure. Also, if there was a $x \in W$ whose orbit was not dense in $W$, we could take its closure as $W^{\prime}$ and that would contradict the minimality of $W$. The sufficiency is because if there was a $W^{\prime} \subset W, W^{\prime} \neq W$, closed and such that $f\left(W^{\prime}\right) \subset W^{\prime}$, we would take $x \in W \backslash W^{\prime}$ and $x^{\prime} \in W^{\prime}$, but the orbit of $x^{\prime}$ would have $x$ as an accumulation point $(x$ is dense in $W$ ), hence we should have $x \in W^{\prime}$, a contradiction.

Therefore, Zorn's lemma gives us a non-empty minimal $Z$ and $x \in Z$, which is dense in $Z$. This $x$ is topologically recurrent.

We could have proved this theorem using theorem 2.4.3. It would have left us exactly under the hypothesis of theorem 2.4.5. Since the invariant measure $\mu$ we would make use of is a probability, every point in a set $G \subset \bar{M}, \mu(G)=\mu(\bar{M})=1$, is topologically recurrent. Of course, $G \neq \varnothing$, so there exists at least one $x \in G$. This $x$ is topologically recurrent.

The following topic should be Birkhoff's ergodic theorem. To continue on this track, the reader can jump to section 3.1.

\subsection{Topological Dynamics}

Until now, we assumed the reader was used to basic topology.

First, we present some Baire category theory. The purpose is to develop a notion of what is small (or large, by taking the complement) in topology. The analogy with the notion of small (large) in measure theory, i.e., with null (full) measure, should be kept in mind.

A first naive guess of what is topologically large is density. But this property does not even survive to finite intersections (nor its complement, the analogous concept for small, does survive to finite unions) since in $\mathbb{R}$ with the standard topology $\mathbb{Q}$ and $\mathbb{Q}+\sqrt{2}$ are dense but their intesection, $\varnothing$, is not.

To educate our notion of what is meant to be small (or large, by taking the complement) during this text, we prescribe that a collection of small sets $\mathcal{S}$ should accomplish the following: given $X$, the collection $\mathcal{S} \subset \mathcal{P}(X)$ should be such that (i) $\varnothing \in \mathcal{S}$, (ii) $A, B \subset X, A \subset B, B \in \mathcal{S} \Rightarrow A \in \mathcal{S}$, (iii) $A_{n} \in \mathcal{S}, \forall n \geq 0 \Rightarrow \cup_{n \geq 0} A_{n} \in \mathcal{S}$. 
Note that under the more general property (iii') that $\mathcal{S}$ is closed for finite union and the additional property (iv') that if $A \subset X$, then one, and only one, between $A$ and $X \backslash A$ should be in $\mathcal{S}$, we would have that the collection $\mathcal{S}$ (satisfying (i),(ii), (iii') and (iv')) is a so-called maximal ideal on $\mathcal{P}(X)$. When the collection $\mathcal{S}$ satisfies (i), (ii), (iii) and (iv') its is called a countably complete (or $\sigma$-complete) maximal ideal on $\mathcal{P}(X)$.

Clearly, the notion of small in measure theory fits the presented (i,ii,iii) specification. On the other hand, the binary condition (iv') will not, in general, apply to it (except for $\{0,1\}$-valued measures), since a set $A$ and its complement $X \backslash A$ can both have measure 0.5 (that is, $A$ is not small, nor large, i.e., $A$ is a set "in between"). As to be presented next, both remarks also will apply to our notion of small in topology.

Definition 2.5.1. Let $(X, \tau)$ be a non-empty topological space. A set $A \subset X$ is said to be:

(i) nowhere dense if int $(\bar{A})=\varnothing$.

(ii) meager (or of first Baire category) if it can be written as a countable union of nowhere dense sets.

(iii) comeager (or residual) if $X \backslash A$ is meager.

(iv) non-meager (or of second Baire category) if it is not meager.

Examples of nowhere dense sets in $\mathbb{R}$ with the standard topology are finite sets, $\{0\} \cup\{1 / n: n \geq 0\}$ (countable) and ternary Cantor set (uncountable).

Proposition 2.5.2 (Properties of nowhere dense sets). Let $(X, \tau)$ be a non-empty topological space, $B, B_{1}, \ldots, B_{k}$ be nowhere dense subsets of $X, C \subset B$ and $A \subset X$. Then:

(o) $A$ is nowhere dense $\Leftrightarrow \forall U \in \tau, U \neq \varnothing, \exists V \in \tau, V \neq \varnothing$, such that $V \subset U$ and $A \cap V=\varnothing$

(i) $\varnothing$ is nowhere dense

(ii) $C$ is nowhere dense

(iii') $\cup_{j=1}^{k} B_{j}$ is nowhere dense

Proof. (o) ( $\Rightarrow$ ) Given $U \in \tau$, if $A \cap V \neq \varnothing, \forall V \subset U$, we would have $U \subset \bar{A}$, a contradiction since $\operatorname{int}(\bar{A})=\varnothing$.

$(\Leftarrow)$ For a contradiction, suppose $\operatorname{int}(\bar{A}) \neq \varnothing$, then $\exists U \in \tau, U \subset \bar{A}$. On the other hand, by hypothesis, we can find $V \subset U$ such that $A \cap V=\varnothing$. We claim that $\bar{A} \cap V=\varnothing$. Since $V \neq \varnothing$, $\exists v \in V \subset U, v \notin \bar{A}$, a contradiction with $U \subset \bar{A}$. The claim holds because if there was a $z \in \bar{A} \cap V$, it should be that $z \in \partial A \cap V$ (since $A \cap V=\varnothing$ ), but taking a neighborhood $W \subset V$ of $z$, we can obtain, by the definition of $\partial A, p \in A \cap W \subset A \cap V=\varnothing$, a contradiction.

(i) $\operatorname{int}(\bar{\varnothing})=\varnothing$

(ii) $\operatorname{int}(\bar{B}) \subset \operatorname{int}(\bar{A})=\varnothing$

(iii') If $B_{1}$ and $B_{2}$ are nowhere dense, by (o), (a) $\forall U_{1} \in \tau, U_{1} \neq \varnothing, \exists V_{1} \subset U_{1}$ such that $V_{1} \cap B_{1}=\varnothing$ and (b) $\forall U_{2} \in \tau, U_{2} \neq \varnothing, \exists V_{2} \subset U_{2}$ such that $V_{2} \cap B_{2}=\varnothing$. In particular, given $U \in \tau, U \neq \varnothing$, and $V_{2}$ obtained as above from $U_{2}:=U$, we can find $V_{1}$ obtained as above from $U_{1}:=V_{2}$. Denote $V:=V_{1}$. Then $V \subset V_{2} \subset U, V \neq \varnothing, V_{2} \cap B_{2}=\varnothing, V_{1} \cap B_{1}=V \cap B_{1}=\varnothing \Rightarrow\left(V_{2} \cap B_{2}\right) \cup\left(V \cap B_{1}\right)=\varnothing \Rightarrow$ $V \cap\left(B_{1} \cup B_{2}\right)=\left(V \cap B_{2}\right) \cup\left(V \cap B_{1}\right) \subset\left(V_{2} \cap B_{2}\right) \cup\left(V \cap B_{1}\right)=\varnothing$. Therefore $B_{1} \cup B_{2}$ satisfy the characterization presented in (o). So $B_{1} \cup B_{2}$ is nowhere dense. By finite induction, $\cup_{j=1}^{k} B_{j}$ is also nowhere dense.

In conclusion, the collection of nowhere dense sets satisfy conditions (i), (ii) and (iii') presented in the fourth and fifth paragraphs of this section.

Property (iii) is not satisfied. For example, in $\mathbb{R}$ with the standard topology, $\mathbb{Q}$ is not nowhere dense (in fact, it is (everywhere) dense), but is a countable union of nowhere dense sets (the rational singletons). Therefore nowhere dense set are not yet the desired notion of topologically small.

Now we move into meager sets. Examples are the ones given for nowhere dense sets, as well as $\mathbb{Q}$ or $\cup_{n \geq 0}\left(\left\{q_{n}\right\}+C\right)$, where $q_{n}$ 's are the enumerated rationals and $C$ the ternary Cantor set.

Proposition 2.5.3 (Properties of meager sets). Let $(X, \tau)$ be a non-empty topological space, B, $B_{1}, \ldots, B_{k}, \ldots$ be meager subsets of $X$ and $C \subset B$. Then: 
(i) $\varnothing$ is meager

(ii) $C$ is meager

(iii) $\cup_{j=1}^{\infty} B_{j}$ is meager

Proof. (i) $\varnothing$ is nowhere dense, hence meager.

(ii) Write $B=\cup_{n \geq 0} B_{n}$, where the $B_{n}$ 's are nowhere dense sets. Since $C \subset B, C=C \cap B=$ $C \cap\left(\cup_{n \geq 0} B_{n}\right)=\cup_{n \geq 0}\left(C \cap B_{n}\right)$, and the $\left(C \cap B_{n}\right)$ 's are nowhere dense by the last proposition.

(iii) Follows because the countable union of countable sets is countable, hence the index set of the new union can be reduced to a countable union over the nowhere dense sets forming the $B_{n}$ 's.

In conclusion, meager sets are the notion of topologically small we should stick with. On the other hand, comeager sets are the notion of topologically large for us. Examples are the complements of those given before.

As we will see in a while, the notion of comeager sets is even better suited to some spaces, which are still quite general. Before addressing this important issue, we explore comeager sets a little further.

By the definition of comeager sets and the previous proposition, (i) the whole space $X$ will be comeager, (ii) they will be closed for taking larger sets and (iii) they will be closed for countable intersection. Additionally, the following characterization is also useful.

Proposition 2.5.4 (Comeager sets characterization). Let $(X, \tau)$ be a non-empty topological space and $A^{\mathrm{c}}$ be a subset of $X$.

Then $A^{\mathrm{c}}$ is comeager $\Leftrightarrow A^{\mathrm{c}}$ contains a countable intersection of open dense sets.

Proof. $(\Rightarrow)$ Let $A=\cup_{n \geq 0} A_{n}$ ( $A_{n}$ 's nowhere dense sets) be a meager set. If $B$ is nowhere dense, then $\bar{B}$ is also nowhere dense, directly from the definition. Hence $\cup_{n \geq 0} \bar{A}_{n}$ is still meager (it is contained in $\bar{A}$, which not necessarily meager, e.g., $A=\mathbb{Q}$ ). Therefore, $A \subset \cup_{n \geq 0} \overline{A_{n}}$. By taking complements, $A^{\mathrm{c}} \supset \cap_{n \geq 0} \overline{A_{n}}{ }^{\mathrm{c}}$, where the $\overline{A_{n}}{ }^{\mathrm{c}}$ 's are open dense sets. A given $\overline{A_{n}{ }^{\mathrm{c}}}$ is dense because, once $A_{n}$ is nowhere dense, given $U \in \tau, U \neq \varnothing, \exists V \in \tau, V \neq \varnothing, V \subset U, V \cap A=\varnothing \Rightarrow V \subset A_{n}{ }^{\mathrm{c}} \Rightarrow{A_{n}}^{\mathrm{c}} \cap U \neq \varnothing$.

$(\Leftarrow)$ It follows because the countable intersection of open dense sets, itself, is already comeager. For instance, let $B=\cap_{n \geq 0} B_{n}$ ( $B_{n}$ 's open dense sets), then $B^{\mathrm{c}}=\cup_{n \geq 0} B_{n}{ }^{\mathrm{c}}$ ( $B_{n}{ }^{\mathrm{c}}$ 's closed nowhere dense sets). A given $B_{n}{ }^{c}$ is nowhere dense because $B_{n}{ }^{c}=\overline{B_{n}{ }^{c}} \Rightarrow \operatorname{int}\left(\overline{B_{n}{ }^{c}}\right)=\operatorname{int}\left({B_{n}}^{c}\right)=\varnothing$, since $B_{n}{ }^{\mathrm{c}}$ had interior, that would prevent $B_{n}$ from being dense. As such, $B$ is comeager.

Lastly, about non-meager sets. It should be noted that nonmeager sets mean being not small, but that does not imply being large. For example, in $\mathbb{R}$ with the standard topology, $[0, \infty)$ is non-meager (non small) but not comeager (large), since its complement is not meager (small).

Now we address when the idea of topological small and large constructed until here has even more significance. Natural questions should have arisen: "Okay, the property of being non-meager implies nothing about the property of being comeager. Does the property of being comeager imply the property of being non-meager? In other words, can something be large but small at the same time? Or even more drastic can the whole space $X$ be meager (small)?" These and many other natural suppositions have the expected answer under the circumstances of the following theorem.

Before the theorem, an auxiliary proposition.

Proposition 2.5.5 (Baire spaces). Let $(X, \tau)$ be a non-empty topological space. Then the following are equivalent:

(i) every non-empty open set is non-meager (in particular, (a) $X$ is non-meager and (b) meager sets have empty interior)

(ii) countable intersection of open dense sets is dense

(iii) comeager sets are dense

In case any of the above holds, $(X, \tau)$ is called a Baire space. 
Proof. (i $\Rightarrow$ ii) We prove the contrapositive. Let $A=\cap_{n \geq 0} O_{n}$ ( $O_{n}$ 's open dense sets) be not dense. Then $\exists U \in \tau, U \neq \varnothing$ such that $A \cap U=\varnothing$. Therefore $U \subset A^{c}=\cup_{n \geq 0} O_{n}{ }^{c}\left(O_{n}{ }^{c}\right.$ 's closed nowhere dense sets). So $U$ in contained in a meager set and should be an open meager set, as desired.

(ii $\Rightarrow$ iii) As we have already characterized, comeager sets contain a countable intersection of dense open sets. By the hypothesis, this intersection is dense and so should be a comeager set.

(iii $\Rightarrow$ i) Take $U \in \tau, U \neq \varnothing$. If $U$ was meager, $U^{c}$ would be comeager but not dense (as long as it does not intersect the open set $U$ ), a contradiction. So $U$ should be non-meager.

Theorem 2.5.6 (Baire category theorem). Let $(X, \tau)$ be a non-empty topological space. If $(X, \tau)$ is a complete metrizable space or locally compact Hausdorff, then it is Baire.

Proof. In both cases, we want to verify property (ii) of proposition 2.5.5. For such, let $\left(U_{n}\right)_{n \geq 0}$ be a family of open dense sets. To check that $\cap_{n \geq 0} U_{n}$ is dense, we let $U$ be an arbitrary open set, and then prove that $\left(\cap_{n \geq 0} U_{n}\right) \cap U \neq \varnothing$.

We argument first for complete metrizable spaces. Since $U \cap U_{0}$ is an open non-empty set $\left(U_{0}\right.$ is dense), we can find a ball $B_{0}$ such that $\overline{B_{0}} \subset U \cap U_{0}$ whose center is $x_{0}$ and whose radius is $r_{0}<1 / 2$. Since $B_{0} \cap U_{1}$ is an open non-empty set ( $U_{1}$ is dense), we can find a ball $B_{1}$ such that $\overline{B_{1}} \subset B_{0} \cap U_{1}$ whose center is $x_{1}$ and whose radius is $r_{1}<1 / 3$. In general, since $B_{n-1} \cap U_{n}$ is an open non-empty set $\left(U_{n}\right.$ is dense), we can find a ball $B_{n}$ such that $\overline{B_{n}} \subset B_{n-1} \cap U_{n}$ whose center is $x_{n}$ and whose radius is $r_{n}<1 / n+2$.

We say that $\left(x_{n}\right)_{n \geq 0}$ is Cauchy. Note that (a) $\forall \epsilon>0, \exists N \geq 0$ such that $r_{n}<\epsilon / 2$ and (b) $\overline{B_{0}} \supset B_{0} \supset \overline{B_{1}} \supset B_{1} \ldots \supset \overline{B_{i-1}} \supset B_{i-1} \supset \overline{B_{i}} \supset B_{i} \supset \overline{B_{i+1}} \supset B_{i+1} \supset \ldots$, so $x_{i} \in B_{j}, \forall i \geq j$. Therefore, $\forall N \leq n<m, x_{m}, x_{n} \in B_{N} \Rightarrow\left|x_{m}-x_{n}\right|<2 r_{N}=2 \epsilon / 2=\epsilon$.

By completeness, $x_{n} \rightarrow x \stackrel{*}{\epsilon} \cap_{n \geq 0} \overline{B_{n}} \stackrel{* *}{=} \cap_{n \geq 0} B_{n} \stackrel{* * *}{ᄃ}\left(\cap_{n \geq 0} U_{n}\right) \cap U$, as desired. Set inequality $(*)$ is because if $x \notin \cap_{n \geq 0} \overline{B_{n}} \Rightarrow x \notin \overline{B_{n_{0}}}$ for some $n_{0}$, but $x_{n} \in \overline{B_{n_{0}}}, \forall n \geq n_{0}$ (by (b)) $\Rightarrow x \in B_{n_{0}}$, a contradiction. Equality $(* *)$ is because if $y \in \cap_{n \geq 0} \overline{B_{n}}$ and $y \notin \cap_{n \geq 0} B_{n} \Rightarrow y \notin B_{n_{0}}$ for some $n_{0}$ $\Rightarrow y \notin \overline{B_{n_{0}+j}}, \forall j>0$ (by (b)) $\Rightarrow y \notin \cap_{n \geq 0} \overline{B_{n}}$, a contradiction - the converse set inequality is trivial. Set inequality $(* * *)$ is because, for each $n \geq 0, B_{n}$ is a subset of $U_{n}$ and of $U$.

The argument for locally compact Hausdorff is similar. By local compactness, $\forall x \in X, U \in \tau$ neighborhood of $x, V \in \tau$ neighborhood of $x$ such that $\bar{V} \subset U$ and $\bar{V}$ compact.

As before, since $U \cap U_{0}$ is an open non-empty set ( $U_{0}$ is dense), we can find an open set $V_{0}$ such that $\overline{V_{0}} \subset U \cap U_{0}$. Since $V_{0} \cap U_{1}$ is an open non-empty set ( $U_{1}$ is dense), we can find an open set $V_{1}$ such that $\overline{V_{1}} \subset V_{0} \cap U_{1}$. In general, since $V_{n-1} \cap U_{n}$ is an open non-empty set ( $U_{n}$ is dense), we can find an open set $V_{n}$ such that $\overline{V_{n}} \subset V_{n-1} \cap U_{n}$. We obtain $\overline{V_{0}} \supset V_{0} \supset \overline{V_{1}} \supset V_{1} \ldots \supset \overline{V_{i-1}} \supset V_{i-1} \supset \overline{V_{i}} \supset$ $V_{i} \supset \overline{V_{i+1}} \supset V_{i+1} \supset \ldots$

On Hausdorff spaces the intersection of non-empty compact sets is not empty. Therefore $\exists y \epsilon$ $\cap_{n \geq 0} \overline{V_{n}}=\cap_{n \geq 0} V_{n} \subset\left(\cap_{n \geq 0} U_{n}\right) \cap U$. Every justification here is analogous those used before.

The classic counter example here is $X=\mathbb{Q}$ with the standard topology. In this case, $X$ is still meager, which happens because this topological space is not complete.

A curious takeaway is the first characterization of Baire spaces in proposition 2.5.5. It states that open sets can be not just a concept of proximity but can also be endowed with "largeness" (with respect to notion of large derived from the topology/concept of proximity defined by themselves).

Finally, as discussed, when $(X, \tau)$ is a Baire space, the idea of topological small and large herein developed is even better suited. Hence, in this context, we adopt the following notation: if a property $P$ holds for every $x$ in a comeager set, we say $P$ is true $\tau$-almost always (abbreviated $\tau$-a.a.), or for $\tau$-almost all $x \in X$ (abbreviated $\tau$-a.a. $x \in X$ ).

Now, we present some basic topological dynamics concepts.

A first concept is topological recurrence, already introduced in theorem 2.4.5: given a topological space $(X, \tau)$ and a dynamical system $f: X \rightarrow X, x \in X$ is said to be recurrent (through $f$ ) if there exists a natural subsequence $\left(n_{k}\right)_{k \geq 0}$ going to infinity such that $f^{n_{k}}(x) \stackrel{k}{\rightarrow} x$. If a recurrent point exits, $f$ is said to have a recurrent point. 
Another topological dynamics concept is transitivity of a system: given a topological space $(X, \tau)$, a dynamical system $f: X \rightarrow X$, is said to be transitive if there exists a point $x \in X$ such that the set of its forward orbit $\left\{f^{n}(x): n \geq 0\right\}$ is dense in $X$. One such $x \in X$ is a called transitive point.

Note that a stronger property than $x \in X$ being a transitive point is: $\forall U \in \tau, N \geq 0, \exists n \geq N$ such that $f^{n}(x) \in U$.

A close related definition (sometimes also named 'transitivity') is the one we call topological irreducibility: given a topological space $(X, \tau)$, a dynamical system $f: X \rightarrow X$, is said to be topologically irreducible if $\forall U, V \in \tau, U, V \neq \varnothing, \exists n \geq 0$ such that $f^{n}(U) \cap V \neq \varnothing$.

Definitions transitivity and topological irreducibility are independent in the sense that, at this level of generality, one does not imply the other. However, the following proposition provides conditions in which they relate.

Proposition 2.5.7 (Transitivity and irreducibility). If $(X, \tau)$ is a topological space with no isolated points, then every topologically irreducible system is also transitive. On the other hand, If $(X, \tau)$ is separable topological space and $X$ is non-meager, then every transitive system is also topologically irreducible.

If the dynamical system was invertible, we could have addressed backward recurrence and transitivity by making simple adaptations.

Now we want to discuss the relation between recurrent and transitive points. The naive guess is that every transitive point is recurrent. The converse relation obviously does not hold (e.g., fixed points).

The naive guess will not hold in the following examples: (a) $X=\{1,2,3\}$ and $f(1)=2, f(2)=$ $3, f(3)=2($ consider $x=1),($ b) $X=\{1,2, \ldots\}$ and $f(x)=x+1$ (consider $x=1),($ c) $X=\{1 / n: n \geq 1\}$ (not compact) and $f(1 / n)=1 / n+1$ (consider $x=1 / 1)$ and (d) $X=\{-1\} \cup[0,1]$ and $f(x)=x+\sqrt{2} / 2$ $(\bmod 1)$, when $x \in[0,1), f(-1)=0$ and $f(1)=1$ (consider $x=-1)$.

We can prevent those counterexamples by requiring that $X$ doe not have isolated points.

Proposition 2.5.8 (Transitivity implies recurrence). Let $X$ be a metric space with no isolated points and $f: X \rightarrow X$ a continuous dynamical system. Then every transitive point is a recurrent point.

Proof. Let $x$ be a transitive point and $E=\left\{f^{n}(x): n \geq 0\right\}$ the dense set of its orbit. We want to show $x$ recurs.

Fix $\epsilon_{0}>0$. Since $x$ is not isolated, choose a sequence $\left(y_{k}\right)_{k \geq 0}$ in $X$ such that $y_{k} \rightarrow x, 0<d\left(y_{0}, x\right)<$ $\epsilon_{0}$ and $0<d\left(y_{k}, x\right)<\min \left\{d\left(y_{k-1}, x\right) / 2, \epsilon_{0} / 2^{k}\right\}, \forall k \geq 1$.

For each $k \geq 0$, with $y_{k} \in B_{\epsilon_{0}}(x)$, we can find $0<\delta_{k}<d\left(y_{k}, x\right) / 2$ so that $B_{\delta_{k}}\left(y_{k}\right) \subset B_{\epsilon_{0}}(x)$ and $B_{\delta_{k}}\left(y_{k}\right) \cap\left(\cup_{j=0}^{k-1} B_{\delta_{j}}\left(y_{j}\right)\right)=\varnothing$. In other words, balls $D_{k}=B_{\delta_{k}}\left(y_{k}\right)$ are pairwise disjoint and contained in $B_{\epsilon_{0}}(x)$.

For $k=0$, choose in $E$ an element $f^{n_{0}}(x) \in D_{0}$.

For $k=1$, choose in $E$ an element $f^{j}(x) \in D_{1}$. If $j \leq n_{0}$, choose in $E$ a (new) element $f^{j^{\prime}}(x) \epsilon$ $D_{1}{ }^{\prime}=B_{\delta_{1}}{ }^{\prime}\left(y_{1}\right)$, where $\delta_{1}{ }^{\prime}=d\left(f^{j}(x), y_{1}\right) / 2$. If $j^{\prime} \leq n_{0}$, choose in $E$ a (new) element $f^{j^{\prime \prime}} \in D_{1}{ }^{\prime \prime}=$ $B_{\delta_{1}{ }^{\prime \prime}}\left(y_{1}\right)$, where $\delta_{1}{ }^{\prime \prime}=d\left(f^{j^{\prime}}(x), y_{1}\right) / 2$. By proceeding this way, in finite many trials we will end up choosing an element $f^{j^{*}}(x) \in D_{1}$ such that $j^{*}>n_{0}$. Set $n_{1}:=j^{*}$.

By reproducing the argument we obtain the desired subsequence $\left(n_{k}\right)_{k \geq 0}$ such that $f^{n_{k}}(x) \stackrel{k}{\rightarrow}$ $x$.

Remark. The following result is stated here to support theorem 3.2.1. The reader may skip it in a first occasion and come back later.

Proposition 2.5.9. Let $X$ be a compact metric space (hence complete and separable, i.e, with a countable basis of open sets), $f: X \rightarrow X$ a continuous dynamical system such that there exists a transitive and recurrent point $x_{0}$. Suppose, additionally, that $X$ is infinite. Then $X$ can have no isolated point and, in particular, every (other) transitive point has to be recurrent. 
Proof. Suppose there exists an isolated point $x_{1}$.

If $x_{0}$ was isolated, by recurrence, it should be periodic, thus not transitive (because $X$ is infinite). So $x_{0}$ is not isolated.

Now, by transitivity of $x_{0}$, and isolation of $x_{1}$, there exists $m_{1} \geq 1$ such that $f^{m_{1}}\left(x_{0}\right)=x_{1}$. But $\exists\left(n_{k}\right)_{k \geq 0}$ a increasing natural sequence going to infinity such that $f^{n_{k}}\left(x_{0}\right) \stackrel{k}{\rightarrow} x_{0} \Rightarrow f^{n_{k}+m_{1}}\left(x_{0}\right) \stackrel{k}{\rightarrow}$ $f^{m_{1}}\left(x_{0}\right) \Rightarrow f^{n_{k}}\left(f^{m_{1}}\left(x_{0}\right)\right) \stackrel{k}{\rightarrow} f^{m_{1}}\left(x_{0}\right) \Rightarrow f^{n_{k}}\left(x_{1}\right) \stackrel{k}{\rightarrow} x_{1}$. The sequence $\left(f^{n_{k}}\left(x_{1}\right)\right)_{k \geq 0}$ is pairwise distinct (otherwise $x_{0}$ would be periodic and not transitive). Thus $x_{1}$ can not be isolated - a contradiction.

Next proposition shows that, under adequate conditions, whenever there exists a transitive point and recurrent point, the set of transitive points will be topologically large. In the following, a Polish space refers to a complete separable (i.e., admitting a countable basis of open sets) metrizable space.

Proposition 2.5.10 (Transitivity is topologically large). Let $(X, \tau)$ be Polish space and $f: X \rightarrow X$ be a continuous dynamical system. If there exists a transitive and recurrent point $z_{0}$, the set $E$ of transitive points is comeager.

Proof. If there is any isolated point $r \in X$, it follows that $\exists v \in\left\{f^{n}\left(z_{0}\right): n \geq 0\right\} \cap\{r\}$. The point $v$ is recurrent, because $v=f^{q}\left(z_{0}\right)$ for some $q \geq 0$ and $\exists n_{k} \nearrow \infty$ such that $f^{n_{k}}\left(z_{0}\right) \rightarrow z_{0}$, so $f^{n_{k}}(v)=f^{n_{k}}\left(f^{q}\left(z_{0}\right)\right)=f^{q}\left(f^{n_{k}}\left(z_{0}\right)\right) \rightarrow f^{q}\left(z_{0}\right)=v$. Therefore $\left\{f^{n}(v): n \geq 0\right\}=\left\{f^{n}\left(z_{0}\right): n \geq 0\right\}$ is a finite dense set. As a consequence, it coincides with $X$ and so every point in $X$ is transitive.

If there is no isolated point, we argue as follows.

Metric spaces are separable if, and only if, are second countable (i.e., admit a countable basis for the topology). So let $\left(U_{j}\right)_{j \geq 0}$ be a basis of open sets for $\tau$. In particular, $\forall U \in \tau, \exists j \geq 0$ such that $U_{j} \subset U$.

We can then write $E=\cap_{j \geq 0} \cup_{n \geq 0} f^{-n}\left(U_{j}\right)$. For instance, $f^{-n}\left(U_{j}\right)$ is the set of points arriving in $U_{j}$ after $n$ iterates, $G_{j}=\cup_{n \geq 0} f^{-n}\left(U_{j}\right)$ is the set of points eventually arriving in $U_{j}$ and $E=\cap_{j \geq 0} G_{j}$ is the set of points eventually arriving in every open set.

Since $f$ is continuous, $f^{-n}\left(U_{j}\right)$ 's are open, and so are the $G_{j}$ 's. So what is left, to check that $E$ is comeager, is to check that the $G_{j}$ 's are themselves dense sets.

Given a fixed $G_{j}$, we want to check it intersects each of the $U_{k}$ 's. Fix one such $U_{k}$. The set $O$ of the forward orbit of $z_{0}$ is infinite, otherwise it would not be dense (since we are in a metric space with no isolated points). Given an open set $U, O \cap U$ should also be infinite, because with a first element on the intersection (exists by density), $x_{1}$, we can take $U \backslash\left\{x_{1}\right\}$ as new open set (in a metric space with no isolated points singletons are always closed) and pick a second element on the intersection (again by density), $x_{2}$, and proceed this way.

Applying these ideas for the open sets $U_{j}$ and $U_{k}$, it is easy to obtain positive natural numbers $n_{0}<n_{1}=n_{0}+t$ such that $x=f^{n_{0}}\left(z_{0}\right) \in U_{k}$ and $f^{t}(x)=f^{n_{0}+t}\left(z_{0}\right)=f^{n-1}\left(z_{0}\right) \in U_{j}$, hence $x \in$ $U_{k} \cap f^{-t}\left(U_{j}\right) \subset U_{k} \cap G_{j}$, as desired.

Remark. The case where $(X, \tau)$ was a Polish space with no isolated points and there exists a transitive $z_{0}$ is a corollary. In this case every transitive point is recurrent.

The next result in this direction is the topological analogue of Birkhoff's ergodic theorem, to be presented in section 3.2 . 


\section{Chapter 3}

\section{Birkhoff's Ergodic Theorems: Measure Theoretical and Topological Analogues}

\subsection{Birkhoff Ergodic Theorem (Measure Theoretical)}

We start this section as a continuation of section 2.4. Again, unless otherwise locally mentioned, this section uses [VO16] as a reference. The organization of topics, statements and proofs are inspired by this text, but not the many interpretations herein discussed, which reflect the view of the author.

At the end of section 2.4, we discussed some recurrence results. Measure recurrence is all about the number of times (e.g., none, one, infinite) a measurable set $A \subset X$ is visited by the orbit of a point $x \in A$. Topological recurrence is all about the number of times an arbitrary neighborhood of a point $x$ is visited by its orbit. Both of these are, of course, analyses from a "typical" (" $\mu$-a.e.") point of view.

But we still have no clue about (a) how many times the orbit of $x$ visits a set $A$, for an arbitrary measurable $A$ (e.g., being $x \notin A$ ), and (b) with what (if any) asymptotic frequency, as time advances ("average visit time"), would the orbit of $x$ visit a set $A$, for an arbitrary measurable $A$. Mathematically, we want to know if $\lim _{n} \frac{\#\left\{j \in \mathbb{N} \cap[0, n-1]: f^{j}(x) \in A\right\}}{n}=\lim _{n} 1 / n \sum_{j=0}^{n-1} \mathcal{X}_{A} \circ f^{j}(x)$ exists and what is its value.

That is when Birkhoff's ergodic theorem comes in. It states that $\lim _{n} 1 / n \sum_{j=0}^{n-1} \psi \circ f^{j}(x)$ is well defined for $\mu$-a.e. $x \in X$ (where $\mu$ is an invariant probability for the dynamical system), for any $\psi \in L^{1}(\mu, \mathbb{R})$ (such as $\psi=\mathcal{X}_{A}$ ). This limit is to be interpreted as the (asymptotic) time average of $\psi$ throughout the $f$-orbit of $x$.

Suppose the average visit time of the $f$-orbit of $x$ with respect to $A$ is well defined. What condition on the system (the dynamical system and its invariant measure) would guarantee that the "average visit time" of $A$ is the measure of $A$ ? That is when the concept of ergodicity comes in.

We will first state the major ergodic theorems and discuss their relation. After that, we will discuss what is ergodicity and how it plugs into the ergodic theorems.

The first ergodic theorem is Von Neumann's ergodic theorem. It provides an answer to our question under more restrictive hypothesis (than other ergodic theorems). Since it is going to be generalized by the next ergodic theorem, no direct proof will be provided.

Theorem 3.1.1 (Von Neumann's ergodic theorem). Let $(X, \mathcal{A})$ be a measurable space, $f: X \rightarrow X$ be a measurable dynamical system and $\mu$ be a probability on $(X, \mathcal{A})$ such that $(f, \mu)$ is an invariant system.

Then, for any $\psi \in L^{2}(\mu, \mathbb{R})$, it holds that $1 / n \sum_{j=0}^{n-1} \psi \circ f^{j} \in L^{2}, \forall n \geq 1$, and $1 / n \sum_{j=0}^{n-1} \psi \circ f^{j} \stackrel{\|\cdot\|_{2}}{\longrightarrow}$ $\dot{P}(\psi)$, where $\dot{P}: L^{2}(\mu, \mathbb{R}) \rightarrow S\left(U_{f}\right)=\left\{\dot{\varphi} \in L^{2}(\mu, \mathbb{R}): \dot{\varphi} \circ f \stackrel{L^{2}}{=} \dot{\varphi}\right\}$ is the orthogonal projection on the linear vector subspace $S\left(U_{f}\right)$. In particular, $\dot{P}(\psi) \in S\left(U_{f}\right)$, hence $\dot{P}(\psi) \circ f=\dot{P}(\psi)$, $\mu$-a.e.. 
Remark. This theorem is the following functional analysis theorem with another clothing:

Theorem 3.1.2. Let $(\mathcal{H},\langle.,\rangle$.$) be a Hilbert space (a vector space equipped with a inner$ product $\langle.,$.$\rangle which induces a norm \|$.$\| such that (\mathcal{H},\|\|$.$) is complete), O: \mathcal{H} \rightarrow \mathcal{H}$ be an isometry and $v \in \mathcal{H}$.

Then $O^{n}(v) \in \mathcal{H}, \forall n \geq 1$, and $1 / n \sum_{j=0}^{n-1} O^{n}(v) \stackrel{\|\cdot\|}{\longrightarrow} \operatorname{Proj}(v), \forall v \in H$, where Proj: $\mathcal{H} \rightarrow S(O)=\{w \in \mathcal{H}: O w=w\}$ is the orthogonal projection on the linear vector subspace $S(O)$.

To connect the dots between these theorems, one should do the following. Remember that, according to proposition 2.1.29, $L^{2}(\mu, \mathbb{R})$ is already a Banach space (a complete normed vector space). Its norm, $\left.\|f\|_{2}=\left(\int|f|^{2} d \mu\right)^{1 / 2}=\left(\int f^{2} d \mu\right)^{1 / 2}=\left(\int f f d \mu\right)\right)^{1 / 2}$ can be induced as $\|f\|_{2}=\sqrt{\langle f, f\rangle}$ from the inner product $\langle f, g\rangle=\int f g d \mu$. Therefore $L^{2}(\mu, \mathbb{R})$ is a Hilbert space.

Use the operator $O$ as $U_{f}: L^{2}(\mu, \mathbb{R}) \rightarrow L^{2}(\mu, \mathbb{R})$, given by $\psi \mapsto \psi \circ f$, the Koopman's operator associated to $f$. It was introduced after proposition 2.4.1, where it was argued that it is an isometry.

Finally, note that $1 / n \sum_{j=0}^{n-1} U_{f}^{n}(\psi)=1 / n \sum_{j=0}^{n-1} \psi \circ f^{j}$.

Remark. Let $(\mathcal{H},\langle.,\rangle$.$) be a Hilbert space and X \subset \mathcal{H}$ be a closed and linear vector subspace of $\mathcal{H}$. Then $Y=X^{\perp}=\{y \in \mathcal{H}:\langle y, x\rangle=0, \forall x \in X\} \subset \mathcal{H}$ is a closed linear vector subspace of $\mathcal{H}$ and, for each $h \in \mathcal{H}$, there exists unique $x_{h} \in X$ and $y_{h} \in Y$ such that $h=x_{h}+y_{h}$.

In the above context, the orthogonal projection $P: \mathcal{H} \rightarrow X$, given by $P(h)=x_{h}$, is linear and such that $\|P\|_{\theta} \leq 1, P^{2}=P,\left\langle h_{1}, P\left(h_{2}\right)\right\rangle=\left\langle P\left(h_{1}\right), h_{2}\right\rangle, \forall h_{1}, h_{2} \in \mathcal{H}$, and $P(h)=\operatorname{argmin}_{x^{\prime} \in X}\left\langle h-x^{\prime}, h-x^{\prime}\right\rangle^{2}$. These properties imply that $\langle h-P(h), P(h)\rangle=\langle P(h-P(h)), h\rangle=\langle P(h)-P(P(h)), h\rangle=\langle P(h)-$ $P(h), h\rangle=\langle 0, h\rangle=0$, in accordance with our geometric intuition.

Even though we are going to proceed to a more general $\mu$-a.e. the last theorem is interesting on its own. It gives a geometrical description of the $L^{2}$ convergence when dealing with $\psi \in L^{2}(\mu, \mathbb{R})$. It also shifts our attention to the fact that being $\dot{\varphi} \circ f=\dot{\varphi}, \mu$-a.e., is a distinguished property for any candidate limit function $\dot{\varphi}$. We are going to keep an eye on this property during the next ergodic theorem.

We now state Birkhoff's ergodic theorem. Before proving this statement, we discuss how Von Neumann's ergodic theorem follows as a corollary.

Theorem 3.1.3 (Birkhoff's ergodic theorem, v1). Let $(X, \mathcal{A})$ be a measurable space, $f: X \rightarrow X$ be a measurable dynamical system and $\mu$ be a probability on $(X, \mathcal{A})$ such that $(f, \mu)$ is an invariant system.

Then $\forall \psi \in L^{1}(\mu, \mathbb{R}), \exists X_{\psi} \in \mathcal{A}, \mu\left(X_{\psi}\right)=1, \lim _{n} 1 / n \sum_{j=0}^{n-1} \psi \circ f^{j}(x)$ exists (denoted $Q(\psi)(x)$ ), $\forall x \in X_{\psi}$.

For a given $\psi \in L^{1}(\mu, \mathbb{R})$, let $\dot{Q}(\psi): X \rightarrow \mathbb{R}$ be an extension of $Q(\psi): X_{\psi} \rightarrow \mathbb{R}$ (herein, taking value 0 out of $X_{\psi}$ ).

We can restate the result as: $\forall \psi \in L^{1}(\mu, \mathbb{R}), \exists X_{\psi} \in \mathcal{A}, \mu\left(X_{\psi}\right)=1,1 / n \sum_{j=0}^{n-1} \psi \circ f^{j} \rightarrow \dot{Q}(\psi), \mu$-a.e. (in $X_{\psi}$ ).

Additionally, the following will also hold:

(i) $\dot{Q}(\psi) \circ f=\dot{Q}(\psi)$, $\mu$-a.e..

(ii) $\int \dot{Q}(\psi) d \mu=\int \psi d \mu$.

(iii) if $\psi \in L^{p}(\mu, \mathbb{R}), p \in[1, \infty]$, then $\dot{Q}(\psi) \in L^{p}(\mu, \mathbb{R})$ and $\|\dot{Q}(\psi)\|_{p} \leq\|\psi\|_{p}$.

Remark. For all $\psi \in L^{1}(\mu, \mathbb{R}), X_{\psi}$ can be chosen as $X_{\psi}^{\text {def }}=\left\{x \in X: \exists \lim _{n} 1 / n \sum_{j=0}^{n-1} \psi \circ f(x)\right\}$. Since $1 / n \sum_{j=0}^{n-1} \psi \circ f^{j}$ is measurable for all $n \geq 1$, then $\limsup _{n} 1 / n \sum_{j=0}^{n-1} \psi \circ f^{j}$ and $\liminf _{n} 1 / n \sum_{j=0}^{n-1} \psi \circ f^{j}$ are also going to be measurable (see 2.1.18). So will be $l=\limsup _{n} 1 / n \sum_{j=0}^{n-1} \psi \circ f^{j}-\liminf _{n} 1 / n \sum_{j=0}^{n-1} \psi \circ f^{j}$. Hence $X_{\psi}^{\text {def }}=l^{-1}(\{0\})$ is a measurable set. Using the findings of the last theorem, we know $X_{\psi} \subset$ $X_{\psi}^{\text {def }}$, so $\mu\left(X_{\psi}^{\text {def }}\right)=1$. Therefore $X_{\psi}$ could be chosen as $X_{\psi}^{\text {def }}$ from the beginning. We will assume $X_{\psi}$ is chosen this way throughout the text.

Now we argue why Von Neumann's theorem follows as a corollary. Consider $\psi \in L^{2}(\mu, \mathbb{R})$ and note that $\psi \in L^{1}(\mu, \mathbb{R})$, since $\mu$ is a probability (see proposition 2.1.33). Due to theorem 3.1.3-(iii), $\dot{Q}(\psi) \in L^{2}(\mu, \mathbb{R})$, so our candidate function for $L^{2}$ convergence, $\dot{Q}(\psi)$, lives in the appropriate space. 
We want to prove $L^{2}$ convergence itself. First suppose $\psi$ is bounded, $|\psi| \leq C$. Then partial averages $1 / n \sum_{j=0}^{n-1} \psi \circ f^{j}$ are dominated in absolute value by $C$ and are $\mu$-a.e. convergent to $\dot{Q}(\psi)$. Thus proposition 2.1.31 guarantees that $1 / n \sum_{j=0}^{n-1} \psi \circ f^{j}$ converges to $\dot{Q}(\psi)$ in $L^{2}$.

If $\psi$ is not bounded, consider the sequence of bounded functions $\psi_{k}=\psi \mathcal{X}_{\{x \in X:|\psi(x)| \leq k\}}, k \geq 0$, and the associated Birkhoff's averages $\dot{Q}\left(\psi_{k}\right), k \geq 0$. By the monotone convergence theorem (see 2.1.21) applied to $\left|\psi-\psi_{k}\right|^{2}$, we have $\left\|\psi-\psi_{k}\right\|_{2} \rightarrow 0$, so for any $\epsilon$, there exists $k_{0}$ such that $\left\|\psi-\psi_{k}\right\|_{2} \leq$ $\epsilon / 3, \forall k \geq k_{0}$.

Moreover, since $\|\cdot\|_{2}$ is a norm and $\mu$ is $f$-invariant: $\left\|\left(\psi-\psi_{k}\right) \circ f^{j}\right\|_{2}=\left(\int\left|\left(\psi-\psi_{k}\right) \circ f^{j}\right|^{2} d \mu\right)^{1 / 2}=$ $\left(\int\left|\left(\psi-\psi_{k}\right)\right|^{2} \circ f^{j} d \mu\right)^{1 / 2}=\left(\int\left|\left(\psi-\psi_{k}\right)\right|^{2} d \mu\right)^{1 / 2}=\left\|\psi-\psi_{k}\right\|_{2}, \forall j \geq 0$. Hence $\left\|1 / n \sum_{j=0}^{n-1}\left(\psi-\psi_{k}\right) \circ f^{j}\right\|_{2} \leq$ $1 / n \sum_{j=0}^{n-1}\left\|\left(\psi-\psi_{k}\right) \circ f^{j}\right\|_{2}=\left\|\psi-\psi_{k}\right\|_{2} \leq \epsilon / 3, \forall n \geq 1, k \geq k_{0}$ (I). So, since $\dot{Q}\left(\psi-\psi_{k}\right)=\dot{Q}(\psi)-\dot{Q}\left(\psi_{k}\right), \mu-$ a.e. (whenever the three of them are defined), $\left\|\dot{Q}(\psi)-\dot{Q}\left(\psi_{k}\right)\right\|_{2}=\left\|\dot{Q}\left(\psi-\psi_{k}\right)\right\|_{2} \leq \epsilon / 3, \forall k \geq k_{0}$ (II), once we consider the bound for the partial averages previously obtained.

Since each $\psi_{k}$ is bounded, $\left\|1 / n \sum_{j=0}^{n-1} \psi_{k} \circ f^{j}-\dot{Q}\left(\psi_{k}\right)\right\|_{2} \rightarrow 0$, as we saw initially. So, for each $k \geq 0$, there exists $n_{0}(k) \geq 1$ such that $\left\|1 / n \sum_{j=0}^{n-1} \psi_{k} \circ f^{j}-\dot{Q}\left(\psi_{k}\right)\right\|_{2} \leq \epsilon / 3, \forall n \geq n_{0}(k)$ (III).

Finally $\left\|1 / n \sum_{j=0}^{n-1} \psi \circ f^{j}-\dot{Q}(\psi)\right\|_{2}=\| 1 / n \sum_{j=0}^{n-1} \psi \circ f^{j}-1 / n \sum_{j=0}^{n-1} \psi_{k} \circ f^{j}+1 / n \sum_{j=0}^{n-1} \psi_{k} \circ f^{j}-\dot{Q}\left(\psi_{k}\right)+$ $\dot{Q}\left(\psi_{k}\right)-\dot{Q}(\psi)\left\|_{2} \leq\right\| 1 / n \sum_{j=0}^{n-1} \psi \circ f^{j}-1 / n \sum_{j=0}^{n-1} \psi_{k} \circ f^{j}\left\|_{2}+\right\| 1 / n \sum_{j=0}^{n-1} \psi_{k} \circ f^{j}-\dot{Q}\left(\psi_{k}\right)\left\|_{2}+\right\| \dot{Q}\left(\psi_{k}\right)-\dot{Q}(\psi) \|_{2} \leq$ $\epsilon / 3+\epsilon / 3+\epsilon / 3=\epsilon$, whenever $k \geq k_{0}$ (see I and II) and $n \geq n_{0}(k)$ (see III). Since we start with $\left\|1 / n \sum_{j=0}^{n-1} \psi \circ f^{j}-\dot{Q}(\psi)\right\|_{2}$, the introduction of terms indexed by $k$ in the estimate is of our choice, so we conveniently choose some $k_{*} \geq k_{0}$ and are left with: $\left\|1 / n \sum_{j=0}^{n-1} \psi \circ f^{j}-\dot{Q}(\psi)\right\|_{2} \leq \epsilon$ whenever $n \geq n_{0}\left(k_{*}\right)$. Therefore $1 / n \sum_{j=0}^{n-1} \psi \circ f^{j}$ converges to $\dot{Q}(\psi)$ in $L^{2}$.

It is left to show that the element $\dot{Q}(\psi)$ to which $1 / n \sum_{j=0}^{n-1} \psi \circ f^{j}$ converges in $L^{2}$, as we have shown, is actually the orthogonal projection $\dot{P}(\psi)$, as stated in theorem 3.1.1.

For instance, when $p=2$, we can use that $L^{2}(\mu, \mathbb{R})$ admits the internal product introduced in section 2.2 to argue that: $\int \dot{Q}(\psi) d \mu=\int \psi d \mu$ (see 3.1.3-(ii)) $\Rightarrow \int(\dot{Q}(\psi)-\psi) d \mu=0 \Rightarrow \int(\dot{Q}(\psi)-$ $\psi) \cdot 1 d \mu=0 \Rightarrow\langle\dot{Q}(\psi)-\psi, 1\rangle=0 \Rightarrow \dot{Q}(\psi)-\psi \perp 1,1 \in S\left(U_{f}\right) \Rightarrow \dot{Q}(\psi)-\psi \in S\left(U_{f}\right)^{\perp}, \dot{Q}(\psi) \in S\left(U_{f}\right)$ (see 3.1.3-(i) $\Rightarrow\langle\dot{Q}(\psi)-\psi, \dot{Q}(\psi)\rangle=0$. Hence $\psi=\dot{Q}(\psi)+(\psi-\dot{Q}(\psi))$, where $\dot{Q}(\psi) \in S\left(U_{f}\right)$ and $\psi-\dot{Q}(\psi) \in S\left(U_{f}\right)^{\perp}$. Therefore $\dot{P}(\psi)=\dot{Q}(\psi)$, $\mu$-a.e., i.e., $\dot{P}(\psi) \stackrel{L^{2}}{=} \dot{Q}(\psi)$, where $\dot{P}(\psi)$ is the orthogonal projection of $\psi$ on the linear vector subspace $S\left(U_{f}\right)$, defined in theorem 3.1.1.

This concludes why Von Neumann's ergodic theorem follows from Birkhoff's ergodic theorem. Now we prove Birkhoff's ergodic theorem itself.

Proof. Let $\psi \in L^{1}(\mu, \mathbb{R})$, without loss of generality, be chosen as a non-negative function. Define $\bar{Q}(\psi)(x)=\limsup _{n} 1 / n \sum_{j=0}^{n-1} \psi \circ f^{j}(x)$ and $\underline{Q}(\psi)(x)=\liminf _{n} 1 / n \sum_{j=0}^{n-1} \psi \circ f^{j}(x)$. We assert that $\int \bar{Q}(\psi) d \mu \leq \int \psi d \mu \leq \int \underline{Q}(\psi) d \mu$. As a consequence, $\underline{Q}(\psi) \leq \bar{Q}(\psi) \Rightarrow \int \underline{Q}(\psi) d \mu \leq \int \bar{Q}(\psi) d \mu \Rightarrow$ $\int \underline{Q}(\psi) d \mu=\int \bar{Q}(\psi) d \mu \Rightarrow \int \bar{Q}(\psi)-\underline{Q}(\psi) d \mu=0 \Rightarrow \bar{Q}(\psi)-\underline{Q}(\psi)=0$, $\mu$-a.e. (in a set denoted $\left.X_{\psi}\right)$, where $Q(\psi)(x)=\lim _{n} 1 / n \sum_{j=0}^{n-1} \bar{\psi} \circ f^{j}(x)$ is well defined. The integral equality (ii), $\int \psi d \mu=$ $\int Q(\psi) d \mu$, also follows from the assertion because $\int \underline{Q}(\psi) d \mu \leq \int \bar{Q}(\psi) d \mu$ will actually coincide at $\int Q(\psi) d \mu$ while $\int \psi d \mu$ is going to be squeezed in between. Invariance is also clear for $Q(\psi), \bar{Q}(\psi)$ and, so, $Q(\psi)$, leading to equality (i). We will prove inequality (iii) separately. For now, it is left to prove the assertion $\int \bar{Q}(\psi) d \mu \leq \int \psi d \mu \leq \int \underline{Q}(\psi) d \mu$.

Fix $M>0$ and $\epsilon>0$. Denote $\bar{Q}(\psi)_{M}(x)=\min \{\bar{Q}(\psi)(x), M\}$ and define the (finite) natural $n(x)=\min \left\{n \geq 1: \bar{Q}(\psi)_{M}(x)-\epsilon \leq 1 / n \sum_{j=0}^{n-1} \psi \circ f^{j}(x)\right\}$. This set, we claim, is non-empty. Due to the definition of limsup, the sequence formed by the Birkhoff averages enters the interval $(\bar{Q}(\psi)(x)-$ $\epsilon, \infty)$, but $\bar{Q}(\psi)_{M}(x)-\epsilon \leq \bar{Q}(\psi)(x)-\epsilon$, leading to our claim. It is also clear that $\bar{Q}(\psi)_{M}$ inherits the invariance of $\bar{Q}(\psi)$ (for such, see what happens when $\bar{Q}(\psi)(x)=M, \bar{Q}(\psi)(x)<M$ and $\bar{Q}(\psi)(x)>$ $M)$.

Therefore, for $n=n(x)$, we have $\bar{Q}(\psi)_{M}(x) \leq 1 / n(x) \sum_{j=0}^{n(x)-1} \psi \circ f^{j}(x)+\epsilon$. Noting that, by invariance, the left-hand side rewrites as $\bar{Q}(\psi)_{M}(x)=1 / n(x) \sum_{j=0}^{n(x)-1} \bar{Q}(\psi)_{M} \circ f^{j}(x)$ and then multiplying by $n(x)$ leads to: 


$$
\sum_{j=0}^{n(x)-1} \bar{Q}(\psi)_{M} \circ f^{j}(x) \leq \sum_{j=0}^{n(x)-1} \psi \circ f^{j}(x)+n(x) \epsilon \quad, \forall x \in X
$$

Now define the sets $A_{n}=\{x \in X: n(x)>n\}, n \geq 0$, and note that $A_{0} \supset A_{1} \supset \ldots$ and $\cap_{n \geq 0} A_{n}=\varnothing$. By the downward continuity of the probability measure $\mu$, there must an $N \geq 1$ such that $\mu\left(A_{n}\right)<$ $\epsilon / M, \forall n \geq N$. We denote $A_{N}=: A$.

The set $A$ marks a (small) set in which it takes long for Birkhoff's average to be close to their limsup. We use this set to create the following truncations (a) $\tilde{\psi}(x)=\psi(x)$ when $x \notin A$ and $\tilde{\psi}(x)=M$ when $x \in A$ (we are not saying that $\tilde{\psi} \leq \psi$ ) and (b) $\tilde{n}(x)=n(x)$ when $x \notin A$ and $\tilde{n}(x)=1$ when $x \in A$ (here, $\tilde{n} \leq n$ ). A crucial improvement should then be noted: $\tilde{n}$ is now uniformly bounded by $N$ (since $x \notin A \Rightarrow n(x) \leq N)$.

A truncated version of formula (3.1) still holds:

$$
\sum_{j=0}^{\tilde{n}(x)-1} \bar{Q}(\psi)_{M} \circ f^{j}(x) \leq \sum_{j=0}^{\tilde{n}(x)-1} \tilde{\psi} \circ f^{j}(x)+\tilde{n}(x) \epsilon \quad, \forall x \in X
$$

, because if $x \notin A$, everything remains the same as in (1), while if $x \in A$, the left-hand side boils to $\sum_{j=0}^{0} \bar{Q}(\psi)_{M} \circ f^{j}(x)=\bar{Q}(\psi)_{M}(x) \leq M$ and right-hand side boils down to $\sum_{j=0}^{0} \tilde{\psi} \circ f^{j}(x)+\epsilon=$ $\tilde{\psi}(x)+\epsilon \stackrel{x \in A}{=} M+\epsilon$. Note, on the left-hand side, we are good to go with $\bar{Q}(\psi)_{M}$ and will not need to bring $\bar{Q}(\tilde{\psi})=\bar{Q}(\tilde{\psi})_{M}$ into the argument.

Also, the following relation can be easily obtained:

$$
\begin{array}{r}
\int \tilde{\psi} d \mu=\int_{A} \tilde{\psi} d \mu+\int_{A^{c}} \tilde{\psi} d \mu=\int_{A} M d \mu+\int_{A^{c}} \psi d \mu \\
\underset{\psi \geq 0}{\leq} M \mu(A)+\int \psi d \mu \leq \int \psi d \mu+M \epsilon / M=\int \psi d \mu+\epsilon
\end{array}
$$

Choose an integer $L>0$ so that $N M / L<\epsilon$ and define the strictly increasing sequence $\left(n_{k}(x)\right)_{k \geq 0}$, where $n_{0}(x)=0, n_{k}(x)=n_{k-1}(x)+\tilde{n}\left(f^{n_{k-1}(x)}(x)\right), k \geq 1$. Note that $n_{1}=\tilde{n}$ and that $1 \leq n_{k}(x)-$ $n_{k-1}(x) \leq N$. Now define $k(x)$ to be the largest non-negative integer $k$ for which $n_{k}(x) \leq L-$ 1. In particular, $0=n_{0}(x)<n_{1}(x)<\ldots<n_{k(x)-1}(x)<n_{k(x)}(x) \leq L-1<L \leq n_{k(x)+1}(x)<$ .... We use exactly this decomposition of $[0, L-1] \cap \mathbb{N}$ into $\left[n_{0}(x), n_{1}(x)-1\right],\left[n_{1}(x), n_{2}(x)-\right.$ $1], \ldots,\left[n_{k-1}(x), n_{k}(x)-1\right],\left[n_{k}(x), n_{k+1}(x)-1\right], \ldots\left[n_{k(x)-1}(x), n_{k(x)}(x)-1\right],\left[n_{k(x)}(x), L-1\right]$ to rewrite the following sum:

$$
\begin{aligned}
\sum_{j=0}^{L-1} \bar{Q}(\psi)_{M} \circ f^{j}(x)= & \sum_{k=1}^{k(x)} \sum_{j=n_{k-1}(x)}^{n_{k}(x)-1} \bar{Q}(\psi)_{M} \circ f^{j}(x)+ \\
& \sum_{j=n_{k(x)}(x)}^{L-1} \bar{Q}(\psi)_{M} \circ f^{j}(x) \quad, \forall x \in X
\end{aligned}
$$

, where the first sum encapsulates all the terms of the decomposition but the last one, which is represented by the last sum (that can be understood as a sort of remainder).

For each index $k$ in (3.4), by doing the change of variable $i=j-n_{k-1}(x)$, we have that: 


$$
\begin{aligned}
& \sum_{j=n_{k-1}(x)}^{n_{k}(x)-1} \bar{Q}(\psi)_{M} \circ f^{j}(x)=\sum_{i=0}^{\tilde{n}\left(f^{n_{k-1}(x)}(x)\right)-1} \bar{Q}(\psi)_{M} \circ f^{i+n_{k-1}(x)}(x) \\
= & \sum_{i=0}^{\tilde{n}(y)-1} \bar{Q}(\psi)_{M} \circ f^{i}(y), y=f^{n_{k-1}(x)}(x) \stackrel{(3.2)}{\leq} \sum_{i=0}^{\tilde{n}(y)-1} \tilde{\psi} \circ f^{i}(y)+\tilde{n}(y) \epsilon \\
= & \sum_{i=0}^{\tilde{n}\left(f^{n_{k-1}(x)}\right)-1} \tilde{\psi} \circ f^{i}\left(f^{n_{k-1}(x)}(x)\right)+\tilde{n}\left(f^{n_{k-1}(x)}(x)\right) \epsilon \\
= & \sum_{j=n_{k-1}(x)}^{n_{k}(x)-1} \tilde{\psi} \circ f^{j}(x)+\tilde{n}\left(f^{n_{k-1}(x)}(x)\right) \epsilon \quad, \forall x \in X .
\end{aligned}
$$

Summing (3.5) for $k$ between 1 and $k(x)$, we obtain an upper bound for the first sum on the right-hand side of (4):

$$
\begin{aligned}
& \sum_{k=1}^{k(x)} \sum_{j=n_{k-1}(x)}^{n_{k}(x)-1} \bar{Q}(\psi)_{M} \circ f^{j}(x) \\
& \leq \sum_{k=1}^{(5)}\left[\left(\sum_{j=n_{k-1}(x)}^{n_{k}(x)-1} \tilde{\psi} \circ f^{j}(x)\right)+\tilde{n}\left(f^{n_{k-1}(x)}(x)\right) \epsilon\right] \\
& =\sum_{j=0}^{n_{k(x)}(x)-1} \tilde{\psi} \circ f^{j}(x)+\epsilon \sum_{k=1}^{k(x)} \tilde{n}\left(f^{n_{k-1}(x)}(x)\right) \\
& \leq \sum_{j=0}^{L-1} \tilde{\psi} \circ f^{j}(x)+\epsilon\left(n_{k(x)}(x)\right), \text { since } n_{k(x)}(x)-1 \leq L-2 \text { and } \tilde{\psi} \geq 0 \\
& \leq \sum_{j=0}^{L-1} \tilde{\psi} \circ f^{j}(x)+\epsilon L, \text { since } n_{k(x)}(x) \leq L .
\end{aligned}
$$

An upper bound of the second sum on the right-hand side of $(3.4), \sum_{j=n_{k(x)}(x)}^{L-1} \bar{Q}(\psi)_{M} \circ f^{j}(x)$, is $N M$, because the sum has $L-1-n_{k(x)}(x)+1=L-n_{k(x)}(x) \leq N$ terms (once, by the maximality of $\left.k(x), n_{k(x)}(x)+N \geq L\right)$, each bounded by $M$. This bound combined with that in (6) gives us: 


$$
\begin{aligned}
& \sum_{j=0}^{L-1} \bar{Q}(\psi)_{M} \circ f^{j}(x) \leq \sum_{j=0}^{L-1} \tilde{\psi} \circ f^{j}(x)+\epsilon L+N M \\
& \Rightarrow L \bar{Q}(\psi)_{M}(x) \leq \tilde{\psi}(x)+\ldots+\tilde{\psi} \circ f^{L-1}(x)+\epsilon L+N M \\
& \Rightarrow \bar{Q}(\psi)_{M}(x) \leq \frac{\tilde{\psi}(x)+\ldots+\tilde{\psi} \circ f^{L-1}(x)}{L}+\epsilon+\frac{N M}{L} \\
& \Rightarrow \int \bar{Q}(\psi)_{M} d \mu \leq \frac{\int \tilde{\psi} d \mu+\ldots+\int \tilde{\psi} \circ f^{L-1} d \mu}{L}+\epsilon+\frac{N M}{L} \\
& \Rightarrow \int \bar{Q}(\psi)_{M} d \mu \leq \frac{L \int \tilde{\psi} d \mu}{L}+\epsilon+\frac{N M}{L} \\
& \text { using }(f, \mu) \text { invariant and proposition } 2.4 .1 \\
& \Rightarrow \int \bar{Q}(\psi)_{M} d \mu \leq \int \tilde{\psi} d \mu+\epsilon+\epsilon \stackrel{(3)}{\leq} \int d \mu+\epsilon+\epsilon+\epsilon \\
& \stackrel{\epsilon \rightarrow 0}{\Rightarrow} \int \bar{Q}(\psi)_{M} d \mu \leq \int \tilde{\psi} d \mu \leq \int \psi d \mu \\
& M \rightarrow \infty \\
& \Rightarrow
\end{aligned}
$$

, which is exactly the left side of the inequality we first claimed.

The second half of the inequality is obtained analogously.

Fix $\epsilon>0$. Since $\psi \geq 0$ and $\underline{Q}(\psi) \geq 0$, a truncation from below is unnecessary. Define $n(x)=$ $\min \left\{n \geq 1: 1 / n \sum_{j=0}^{n-1} \psi \circ f^{j}(x) \leq \underline{Q}(\psi)(x)+\epsilon\right\}$. Now we have:

$$
\sum_{j=0}^{n(x)-1} \underline{Q}(\psi) \circ f^{j}(x) \geq \sum_{j=0}^{n(x)-1} \psi \circ f^{j}(x)-\tilde{n}(x) \epsilon \quad, \forall x \in X .
$$

Defining $A_{n}$ as before, we can still control it's measure for large indexes and we, now, put $A=A_{N}$ so that $\mu\left(A_{n}\right)<\delta, \forall n \geq N$, where $\delta$ is chosen so that $\int_{E} \psi d \mu<\epsilon, \forall E \in \mathcal{A}, \mu(E)<\delta$. One such $\delta$ exists because (a) $\lim _{r \rightarrow \infty} \int_{\{\psi>r\}} \psi d \mu=\int \psi \mathcal{X}_{\{\psi>r\}} d \mu=0$ (dominated convergence theorem) $\Rightarrow \exists R>0$ such that, $\forall r^{\prime} \geq R, \int_{\left\{\psi>r^{\prime}\right\}} \psi d \mu<\epsilon / 2$ and (b) $\int_{E} \psi d \mu=\int_{E \cap\{\psi \leq r\}} \psi d \mu+\int_{E \cap\{\psi>r\}} \psi d \mu \leq \int_{E} r d \mu+\int_{\{\psi>r\}} \psi d \mu=$ $r \mu(E)+\int_{\{\psi>r\}} \psi d \mu \Rightarrow \int_{E} \psi d \mu \leq R \mu(E)+\int_{\{\psi>R\}} \psi d \mu \leq R \mu(E)+\epsilon / 2$ hence smaller then $\epsilon$ whenever $\mu(E)<\epsilon / 2 R=: \delta$. The consequence we are looking for is:

$$
\int \tilde{\psi} d \mu \leq \int \psi d \mu=\int_{A} \psi d \mu+\int_{A^{c}} \psi d \mu \leq \epsilon+\int_{A^{c}} \tilde{\psi} d \mu=\epsilon+\int \tilde{\psi} d \mu .
$$

Finally, define (a) $\tilde{\psi}(x)=\psi(x)$ when $x \notin A$ and $\tilde{\psi}(x)=0$ when $x \in A$ and (so, $\tilde{\psi} \leq \psi$ ) (b) $\tilde{n}(x)=n(x)$ when $x \notin A$ and $\tilde{n}(x)=1$ when $x \in A$ (so, $\tilde{n} \leq n, \tilde{n} \leq N$ ). Using these definitions the following new version inequality (3.8) is obtained:

$$
\sum_{j=0}^{\tilde{n}(x)-1} \underline{Q}(\psi) \circ f^{j}(x) \geq \sum_{j=0}^{\tilde{n}(x)-1} \tilde{\psi} \circ f^{j}(x)-n(x) \epsilon \quad, \forall x \in X .
$$

Let $L$ be any (large) natural number. Define $\left(n_{k}(x)\right)_{k \geq 0}$ and $k(x)$ just as before. We use a slight different decomposition of the following sum: 


$$
\begin{aligned}
\sum_{j=0}^{L-1} \underline{Q}(\psi) \circ f^{j}(x)= & \sum_{k=1}^{k(x)+1} \sum_{j=n_{k-1}(x)}^{n_{k}(x)-1} \underline{Q}(\psi) \circ f^{j}(x) \\
& -\sum_{j=L}^{n_{k(x)+1}(x)-1} \underline{Q}(\psi) \circ f^{j}(x) \quad, \forall x \in X .
\end{aligned}
$$

Combining the last two inequalities just as before, we arrive in:

$$
\begin{aligned}
\sum_{j=0}^{L-1} \underline{Q}(\psi) \circ f^{j}(x) \geq & \sum_{j=0}^{n_{k(x)+1}(x)-1} \tilde{\psi} \circ f^{j}(x)+\epsilon n_{k(x)+1}(x) \\
& -\sum_{j=L}^{n_{k(x)+1}(x)-1} \underline{Q}(\psi) \circ f^{j}(x) \\
& \geq \sum_{j=0}^{L-1} \tilde{\psi} \circ f^{j}(x)+\epsilon L-N \underline{Q}(\psi) \circ f^{j}(x) \quad, \forall x \in X .
\end{aligned}
$$

Integrating and using $\mu$ is $f$-invariant:

$$
\begin{gathered}
L \int \underline{Q}(\psi) d \mu \geq L \int \tilde{\psi} d \mu+L \epsilon-N \int \underline{Q}(\psi) d \mu \\
\Leftrightarrow \int \underline{Q}(\psi) d \mu \geq \frac{L}{(L+N)} \int \tilde{\psi} d \mu+\frac{L}{(L+N)} \epsilon \\
\underset{\text { Nifixed }}{\Rightarrow} \int \underline{Q}(\psi) d \mu \geq \int \tilde{\psi} d \mu+\epsilon \stackrel{(3.9)}{\geq} \int \psi d \mu .
\end{gathered}
$$

This concludes the major part of our proof. It is only left to prove property (iii). For $p \in[1, \infty)$, we proceed as follows (for $p=\infty$ the assertion is straightforward):

$$
\begin{aligned}
& |Q(\psi)|=\left|\lim _{n} 1 / n \sum_{j=0}^{n-1} \psi \circ f^{j}\right|=\lim _{n}\left|1 / n \sum_{j=0}^{n-1} \psi \circ f^{j}\right| \leq \lim _{n} 1 / n \sum_{j=0}^{n-1}\left|\psi \circ f^{j}\right| \\
& \Rightarrow \int|Q(\psi)|^{p} d \mu \leq \int\left(\lim _{n} 1 / n \sum_{j=0}^{n-1}\left|\psi \circ f^{j}\right|\right)^{p} d \mu=\int\left(\liminf _{n} 1 / n \sum_{j=0}^{n-1}\left|\psi \circ f^{j}\right|\right)^{p} d \mu \\
& =\int \liminf _{n}\left(1 / n \sum_{j=0}^{n-1}\left|\psi \circ f^{j}\right|\right)^{p} d \mu \underset{2.1 .22}{\operatorname{thm}} \liminf _{n} \int\left(1 / n \sum_{j=0}^{n-1}\left|\psi \circ f^{j}\right|\right)^{p} d \mu \\
& \stackrel{()^{1 / p}}{\Rightarrow}\|Q(\psi)\|_{p} \leq \liminf _{n}\left\|1 / n \sum_{j=0}^{n-1}\left|\psi \circ f^{j}\right|\right\|_{p} \underset{2.1 .28}{\stackrel{\operatorname{prop}}{\leq}} \liminf _{n} 1 / n \sum_{j=0}^{n-1}\left\|\left|\psi \circ f^{j}\right|\right\|_{p} \\
& =\liminf _{n} 1 / n \sum_{j=0}^{n-1}\left\|\psi \circ f^{j}\right\|_{p} \stackrel{(*)}{=} \liminf _{n} 1 / n \sum_{j=0}^{n-1}\|\psi\|_{p}=\liminf _{n}\|\psi\|_{p}=\|\psi\|_{p} \\
& \Rightarrow\|Q(\psi)\|_{p} \leq\|\psi\|_{p}
\end{aligned}
$$

, where $(*)$ is because $\left\|\psi \circ f^{j}\right\|_{p}=\left(\int\left|\psi \circ f^{j}\right|^{p} d \mu\right)^{1 / p}=\left(\int|\psi|^{p} \circ f^{j} d \mu\right)^{1 / p}=\left(\int|\psi|^{p} d \mu\right)^{1 / p}(\mu$ is f-invariant $)=$ $\|\psi\|_{p}$. The proof is then concluded.

We now discuss the last theorem.

Before all, this result is just what we were looking for in the beginning of this section. It provides 
a pointwise existence of the asymptotic time average of $\psi\left(\right.$ in $\left.L^{1}(\mu, \mathbb{R})\right)$ throughout the $f$-orbit of $x$, for $\mu$-a.e. $x \in X$. The function describing this limit $\dot{Q}(\psi)$ was shown to have even more properties, some of which we discuss next.

In (i), $\dot{Q}(\psi)$ being invariant $\left(\dot{Q}(\psi) \in S\left(U_{f}\right)\right.$, in the previous notation) is natural, because if the limit is defined for $x$ it should be defined for $f(x)$ and have the same value, as long as it is a long term property of the orbit. Therefore, it is natural to expect that $Q(Q(\psi))=Q(\psi)$, once $Q(\psi)$ will be constant over $f$-orbits. This sounds like $Q$ is a projection on $L^{p}(\mu, \mathbb{R})$, and this $(Q \circ Q=Q)$ is precisely the definition of projection on a Banach space. Remember, however, that the usual "geometric content" of a projection is dramatically impaired in this context.

An intuitive imagetic interpretation of the integral equality in (ii) is to think that the space was partitioned in orbits and $\dot{Q}(\psi)$ levels each of them as to be equal to their respective $\psi$ average (whenever it is defined, as it happens on a set of full measure of starting points; but taking value 0 when it is not defined). So, in some sense, in each partition, we are changing (a) integration of $\psi$ by (b) integration of the constant value of the average of $\psi$ (in that partition). As, heuristically, we may think $\mu$ puts equal weight in elements of the same orbit, (a) and (b) would be equal and the contribution of each partition would balance. This imagetic resource is clearly not to be taken literary, as it involves understanding how much $\mu$ weights each orbit and each of its elements and, most dangerously, it involves decomposing the space in orbits.

It is worth noting that Birkhoff's ergodic theorem also relates to the concept of density of a set of natural numbers. The density of a set $E \subset \mathbb{N}$ is the number $d(E)=\lim _{n} \frac{\#(E \cap[0, n-1])}{n}$, whenever it is well defined. The collection of sets in which $d$ is well defined constitutes an algebra and the set function $d$ is a finitely additive measure on this algebra (clearly, it will not be countably additive, since $\mathbb{N}$ is the countable union of singletons, all of which have density 0 ).

Birkhoff's ergodic theorem states that $V_{A}(x)=\left\{j \in \mathbb{N}: f^{j}(x) \in A\right\}$ is an element of this algebra for $\mu$-a.e. $x \in X$ and that $d\left(V_{A}(x)\right)=\lim _{n} \frac{\#\left(V_{A}(x) \cap[0, n-1]\right)}{n}=\lim _{n} \frac{\#\left\{j \in \mathbb{N} \cap[0, n-1]: f^{j}(x) \in A\right\}}{n}=$ $\lim _{n} 1 / n \sum_{j=0}^{n-1} \mathcal{X}_{A} \circ f^{j}(x)=\dot{Q}\left(\mathcal{X}_{A}\right)(x)$, $\mu$-a.e. $x \in X$.

The following version is a useful modification of Birkhoff's ergodic theorem, using compactness and metrizability on $X$ in other to find a set $X_{*}$ (independent of $\psi$ ) in which $\mu$-a.e. convergence occurs for all continuous $\psi$.

Theorem 3.1.4 (Birkhoff's ergodic theorem, v2). Let $\bar{M}$ be a compact metric space, $\left(\bar{M}, \mathcal{B}_{\bar{M}}\right)$ be a measurable space, $f: \bar{M} \rightarrow \bar{M}$ be a measurable dynamical system and $\mu$ be a probability on $\left(\bar{M}, \mathcal{B}_{\bar{M}}\right)$ such that $(f, \mu)$ is an invariant system. Then:

(A) using the notation of theorem 3.1.3, $\exists \bar{M}_{\star} \in \mathcal{B}_{\bar{M}}, \mu\left(\bar{M}_{\star}\right)=1, \forall \psi \in C(\bar{M}, \mathbb{R}), 1 / n \sum_{j=0}^{n-1} \psi \circ f^{j} \rightarrow$ $\dot{Q}(\psi)$, $\mu$-a.e. (in $\bar{M}_{*}$ ). The remaining conclusions of theorem 3.1 .3 also apply.

Rewriting, with $\alpha_{f, n}(x)=1 / n \sum_{j=0}^{n-1} \delta_{f^{j}(x)}$, we have $1 / n \sum_{j=0}^{n-1} \psi \circ f^{j}(x)=\int \psi d \alpha_{f, n}(x) \rightarrow \dot{Q}(\psi)(x)=$ $\lambda_{x}(\psi)$.

(B) It holds that, for all $x \in \bar{M}_{*}, \lambda_{x}$ is a functional in $C(\bar{M}, \mathbb{R})^{*}$, hence it is a signed measure (more precisely, a probability) and we can interchange $\lambda_{x}(\psi)$ with $\int \psi d \lambda_{x}$. Therefore, it all can be summarized as: $\alpha_{f, n}(x) \stackrel{w *}{\longrightarrow} \lambda_{x}, \mu$-a.e. $x \in \bar{M}$ (in $\bar{M}_{*}$ ).

Or, denoting $A(f, x)=\left\{\nu\right.$ Borelian probability: $\alpha_{f, n}(x)=1 / n \sum_{j=0}^{n-1} \delta_{f^{j}(x)}$ accumulates in $\nu$ on the weak* topology $\}: A(f, x)=\left\{\lambda_{x}\right\}$, $\mu$-a.e. $x \in \bar{M}$ (in $\left.\bar{M}_{*}\right)$.

Proof. Let's first prove (A).

Start by noting that, since $\bar{M}$ is compact, then $C(\bar{M}, \mathbb{R}) \subset L^{1}(\mu, \mathbb{R})$, and that, for each $\psi \epsilon$ $C(\bar{M}, \mathbb{R})$, the previous version provides us the set $\bar{M}_{\psi}$, with $\mu\left(\bar{M}_{\psi}\right)=1$, such that $1 / n \sum_{j=0}^{n-1} \psi$ 。 $f^{j}(x) \rightarrow \dot{Q}(\psi)(x), \forall x \in \bar{M}_{\psi}$. When $\bar{M}$ is a compact metric space, $C(\bar{M}, \mathbb{R})$ is separable. This allows us to take a countable $\|\cdot\|_{u}$-dense subset of $C(\bar{M}, \mathbb{R}),\left\{\psi_{k}: k \in \mathbb{N}\right\}$. Defining $\bar{M}_{\star}=\cap_{k \in \mathbb{N}} \bar{M}_{\psi_{k}}$, it is clear that $\mu\left(\bar{M}_{\star}\right)=1$. It is only left to show that, for any $x \in \bar{M}_{\star}$ and $\psi \in C(\bar{M}, \mathbb{R}), \lim _{n} 1 / n \sum_{j=0}^{n-1} \psi \circ f^{j}(x)$ exists.

Let $x$ and $\psi$ be as above and $\epsilon>0$. Choose $\psi_{k}$ such that $\left\|\psi-\psi_{k}\right\|_{u}<\epsilon$. Note that $\limsup _{n} 1 / n \sum_{j=0}^{n-1} \psi \circ$ $f^{j}(x) \leq \limsup _{n} 1 / n \sum_{j=0}^{n-1}\left(\psi_{k} \circ f^{j}(x)+\epsilon\right)=\epsilon+\lim _{n} 1 / n \sum_{j=0}^{n-1} \psi_{k} \circ f^{j}(x)=\dot{Q}\left(\psi_{k}\right)+\epsilon$. Likewise, 
$\liminf _{n} 1 / n \sum_{j=0}^{n-1} \psi \circ f^{j}(x) \geq \liminf _{n} 1 / n \sum_{j=0}^{n-1}\left(\psi_{k} \circ f^{j}(x)-\epsilon\right)=-\epsilon+\lim _{n} 1 / n \sum_{j=0}^{n-1} \psi_{k} \circ f^{j}(x)=\dot{Q}\left(\psi_{k}\right)-\epsilon$. $_{n}$ So $\left|\lim \sup _{n} 1 / n \sum_{j=0}^{n-1} \psi \circ f^{j}(x)-\liminf _{n} 1 / n \sum_{j=0}^{n-1} \psi \circ f^{j}(x)\right| \leq 2 \epsilon$. Therefore the limit $\lim _{n} 1 / n \sum_{j=0}^{n-1} \psi \circ$ $f^{j}(x)$ exists, $\forall x \in \bar{M}_{\star}, \psi \in C(\bar{M}, \mathbb{R})$, as desired.

Now let's prove (B).

First, for $x \in \bar{M}_{\star}, \lambda_{x}(\psi)$ is defined for every $\psi$ varying in $C(\bar{M}, \mathbb{R})$, so $\lambda_{x}$ is, in fact, a functional. The linearity of $\lambda_{x}$ follows from: $(a \psi+\varphi) \circ f^{j}(x)=a \psi \circ f^{j}(x)+\varphi \circ f^{j}(x)$. The continuity of $\lambda_{x}$ follows from its boundedness: clearly $\left|\lambda_{x}(\psi)\right| \leq\|\psi\|_{u} \Rightarrow\left\|\lambda_{x}\right\|_{\theta} \leq 1$. The positivity of $\lambda_{x}$ is also clear. Finally, it is a probability because $\lambda_{x}(\mathbf{1})=1$.

The restatement with weak $*$ convergence is because $\lim _{n} \int \psi d \alpha_{f, n}(x)=\int \psi d \lambda_{x}, \forall \psi \in C(\bar{M}, \mathbb{R})$. The restatement with $M(f, x)$ is because the weak* topology is metrizable, so a convergent sequence can not accumulate elsewhere.

The structure of the first version of Birkhoff's ergodic theorem is to have $\psi \in L^{1}(\mu, \mathbb{R})$ fixed and vary $x \in X_{\psi}$. If, in that context, we wanted to fix some $x$ and vary $\psi$, there would be no guarantee that the Birkhoff's limit would continue well-defined - namely, choosing $\psi$ and $\varphi$ as indicated by the theorem does not prevent $x \in X_{\psi}$ while $x \notin X_{\varphi}$ from happening, for a given $x$.

In the second version, by restricting the hypothesis to $X=\bar{M}$ a compact metric space, we obtain a set $\bar{M}_{*}$ such that $x \in \bar{M}_{*} \Rightarrow x \in X_{\psi}, \forall \psi \in C(\bar{M}, \mathbb{R}) \subset L^{1}(\mu, \mathbb{R})$. This allow us to change perspective: to have $x \in \bar{M}_{*}$ fixed an vary $\psi \in C(\bar{M}, \mathbb{R})$. This perspective allows to talk about functionals, measures and weak* convergence.

It is useful to note that these previous two perspectives are closely related. Consequently, $\dot{Q}(\psi)$, from theorem 3.1.3, and $\lambda_{x}$, from theorem 3.1.4, are counterparts: the first in a framework of a general ambient space $X$, using a parameter $\psi \in L^{1}(\mu, \mathbb{R})$ and an argument $x \in X_{\psi}$; the second in a more restrictive framework of a compact metric ambient space $\bar{M}$, using a parameter $x \in \bar{M}_{\star}$ and an argument $\psi \in C(\bar{M}, \mathbb{R})$.

We will explicitly characterize $\dot{Q}(\psi)$ and $\lambda_{x}$. First, using an additional assumption, which is central to the theory: ergodicity. Second, using a technique called ergodic decomposition.

Ergodicity is a property of an invariant system which involves the concept of invariant measurable sets $\left(A \in \mathcal{A}\right.$ such that $\left.f^{-1}(A)=A\right)$. In fact, the definition to be presented involves the more general concept of essentially invariant measurable sets, namely, those $A \in \mathcal{A}$ such that $\mu\left(A \triangle f^{-1}(A)\right)=0$.

Definition 3.1.5 (Ergodicity). Let $(X, \mathcal{A})$ be a measurable space, $f: X \rightarrow X$ be a measurable dynamical system and $\mu$ be a probability on $(X, \mathcal{A})$ such that $(f, \mu)$ is an invariant system. We say $\mu$ is $f$-ergodic, or that $(f, \mu)$ is an ergodic system, if every essentially invariant measurable set is such that $\mu(A) \in\{0,1\}$.

This means there is no $f$-invariant set on the space $X$ which is not $\mu$-trivial, or, in other words, we can not separate the system in two standalone parts in a non $\mu$-trivial manner. Intuitively, $f$ mess it up well enough (from the point of view of $\mu$ ). This intuition will be improved later.

The following proposition is very useful characterization of ergodicity.

Proposition 3.1.6 (Characterization of ergodicity). Let $(X, \mathcal{A})$ be a measurable space, $f: X \rightarrow X$ be a measurable dynamical system and $\mu$ be a measure on $(X, \mathcal{A})$ such that $(f, \mu)$ is an invariant system. Then the following are equivalent:

(i) $(f, \mu)$ is an ergodic system.

(ii) $\forall \psi \in L^{1}(\mu, \mathbb{R}): \dot{Q}(\psi) \equiv k_{(\psi)}$, $\mu$-a.e., for some $k_{(\psi)} \in \mathbb{R}$.

(iii) $\forall \psi \in L^{1}(\mu, \mathbb{R}): \dot{Q}(\psi) \equiv \int \psi d \mu$, $\mu$-a.e..

(iv) $\forall \dot{\varphi} \in L^{1}(\mu, \mathbb{R})$ which is $f$-invariant (i.e., $\dot{\varphi} \circ f=\dot{\varphi}, \mu$-a.e.): $\dot{\varphi} \equiv k_{(\varphi)}, \mu$-a.e., for some $k_{(\varphi)} \in \mathbb{R}$.

(v) $\forall \dot{\varphi} \in L^{1}(\mu, \mathbb{R})$ which is $f$-invariant (i.e., $\dot{\varphi} \circ f=\dot{\varphi}, \mu$-a.e.): $\dot{\varphi} \equiv \int \dot{\varphi} d \mu$, $\mu$-a.e.. 
In words, $(f, \mu)$ is an ergodic system exactly when every $\dot{Q}(\psi)$ is $\mu$-a.e. a constant $k$ (and by equality (ii) of theorem 3.1.3, $k=\int k d \mu=\int \dot{Q}(\psi) d \mu=\int \psi d \mu$ ), or equivalently, exactly when every invariant $\dot{\varphi}$ is $\mu$-a.e. a constant $k\left(k=\int k d \mu=\int \dot{\varphi} d \mu\right)$, i.e., $S\left(U_{f}\right)$ consists only of constant functions.

Proof. Immediately, (iii) $\Rightarrow$ (ii) and (v) $\Rightarrow$ (iv).

Now, (v) $\Rightarrow$ (iii) and (iv) $\Rightarrow$ (ii), because, by theorem 3.1.3, $\forall \psi \in L^{1}(\mu, \mathbb{R}), \dot{Q}(\psi) \in L^{1}(\mu, \mathbb{R})$ is $f$-invariant, then our hypotheses guarantee that $\dot{Q}(\psi)$ equals $\mu$-a.e., respectively, $\int \dot{Q}(\psi) d \mu=\int \psi d \mu$ (by theorem 3.1.3) and $k$, as desired.

It is left to prove that (ii) $\Rightarrow$ (i) and (i) $\Rightarrow(v)$.

For (ii) $\Rightarrow$ (i), let $A \in \mathcal{A}$, be such that $\mu\left(A \triangle f^{-1}(A)\right)=0$. Since $A$ is essentially invariant, there exists $A^{\prime} \subset A, \mu\left(A^{\prime}\right)=\mu(A)$, and $A^{\prime \prime} \subset A^{\mathrm{c}}, \mu\left(A^{\prime \prime}\right)=\mu\left(A^{\mathrm{c}}\right)$, such that $\dot{Q}\left(\mathcal{X}_{A}\right)$ is defined $\mu$-a.e. taking values 1 for $x \in A^{\prime}$ and 0 for $x \in A^{\prime \prime}$. By hypothesis $\dot{Q}\left(\mathcal{X}_{A}\right)=k \mu$-a.e.. Using 3.1.3-(ii), it is easy to check that $k=\int \mathcal{X}_{A} d \mu=\mu(A)$. Therefore it can only be that $\mu(A)=1$ (and $\left.\mu\left(A^{\prime}\right)=1\right)$ or $\mu(A)=0$ (and $\left.\mu\left(A^{\prime \prime}\right)=1\right)$.

For $(\mathrm{i}) \Rightarrow(\mathrm{v})$, let $\dot{\varphi}$ be a $f$-invariant but not $\mu$-a.e. constant function, i.e., $\exists x_{1} \in \mathbb{R}$, such that $0<\mu\left(\dot{\varphi}^{-1}\left(\left(-\infty, x_{1}\right]\right)\right)<1$ and $0<\mu\left(\dot{\varphi}^{-1}\left(\left(x_{1}, \infty\right)\right)\right)<1$. Let be $A=\dot{\varphi}^{-1}\left(\left(-\infty, x_{1}\right]\right)$. Note that, $f^{-1}(A)=A$, since $x \in f^{-1}(A) \Rightarrow f(x) \in A \Rightarrow \dot{\varphi}(f(x)) \leq x_{1} \Rightarrow \dot{\varphi}(x) \leq x_{1} \Rightarrow x \in A$, conversely $x \in A \Rightarrow \dot{\varphi}(x) \leq x_{1} \Rightarrow \dot{\varphi}(f(x)) \leq x_{1} \Rightarrow f(x) \in A \Rightarrow x \in f^{-1}(A)$. Therefore $A$ is $f$-invariant and $\mu(A) \notin\{0,1\}$, a contradiction.

Therefore, when we start with an ergodic system, conclusions of every version of Birkhoff's ergodic theorem narrow dramatically, as we present bellow (for economy reasons, we adapt only the major convergence statements in each version).

Theorem 3.1.7 (Birkhoff's ergodic theorem with ergodicity, v1). Let $(X, \mathcal{A})$ be a measurable space, $f: X \rightarrow X$ be a measurable dynamical system and $\mu$ be a probability on $(X, \mathcal{A})$ such that $(f, \mu)$ is an ergodic system.

Then $\forall \psi \in L^{1}(\mu, \mathbb{R}), \exists X_{\psi} \in \mathcal{A}, \mu\left(X_{\psi}\right)=1,1 / n \sum_{j=0}^{n-1} \psi \circ f^{j} \rightarrow \int \psi d \mu$, $\mu$-a.e. (in $X_{\psi}$ ).

Theorem 3.1.8 (Birkhoff's ergodic theorem with ergodicity, v2). Let $\bar{M}$ be a compact metric space, $\left(\bar{M}, \mathcal{B}_{\bar{M}}\right)$ be a measurable space, $f: \bar{M} \rightarrow \bar{M}$ be a measurable dynamical system and $\mu$ be a probability on $\left(\bar{M}, \mathcal{B}_{\bar{M}}\right)$ such that $(f, \mu)$ is an invariant and ergodic system. Then:

(A) using the notation of theorem 3.1.7, $\exists \bar{M}_{*} \in \mathcal{B}_{\bar{M}}, \mu\left(\bar{M}_{*}\right)=1, \forall \psi \in C(\bar{M}, \mathbb{R}), 1 / n \sum_{j=0}^{n-1} \psi \circ f^{j} \rightarrow$ $\dot{Q}(\psi) \equiv \int \psi d \mu$, $\mu$-a.e. (in $\bar{M}_{*}$ ).

(B) $\alpha_{f, n}(x) \stackrel{w *}{\longrightarrow} \lambda_{x}=\mu$, or $A(f, x)=\{\mu\}, \mu$-a.e.x $\in X$ (in $\bar{M}_{*}$ ).

This characterization is rich and allows us to reinforce the intuition that ergodicity happens when $f$ mess it up well enough (from the point of view of $\mu$ ). If necessary, with no loss, assume $X$ is a compact metric space, in order to make the story telling more smooth.

The first interpretation is that the $f$-orbit of a $\mu$-typical point $x$ in $X$ spreads over the space in such a manner that, asymptotically, the $\psi$ average along the $f$-orbit of $x$ coincides with $\int \psi d \mu$, for any $\mu$-integrable $\psi$. That is, the set associated to the $f$-orbit of $x$ fills the space $X$ is such a way that it, asymptotically, weights every single piece of space exactly is $\mu$ does. Hence, not willing to make any practical statement, one can use a dynamical system $f$ which is ergodic for $\mu$ in order to approximate $\int \psi d \mu$, for any $\psi \in L^{1}(\mu, \mathbb{R})$.

By the same reasoning, arranging the $f$-orbit of a $\mu$-typical point $x$ in $X$ inside a set (omitting the associated order) makes this set "hardly distinguishable from a $\mu$ countable set-realization" (to be understood heuristically). Note that the set obtained from any finite truncation of an orbit could be very unlikely drawn from $\mu$, but as time (set cardinality) progresses, finite distributional idiosyncrasies are dominated by long term correctness.

However, a $\mu$-typical $f$-orbit obtained from an ergodic system $(f, \mu)$ would not indistinguishable from a sequence of independent draws from the probability $\mu$. In any dynamical system the orbit of a point is predetermined, so there is no independence whatsoever. Some examples, as the irrational rotation in the circle (see 3.1.10), can make this issue noticeable. 
Another important physical interpretation for ergodicity is that allows time averages and space averages to coincide, in the sense the time a $\mu$-typical particle $x \in X$ spends inside a set $A$ of the state space $X$, as the system acts, is exactly the $\mu$-measure of $A$.

The following are examples of ergodic systems.

Example 3.1.9 (Unilateral Bernoulli shift). Let $(X, \mathcal{A}, \mu)$ be a probability space where and $\left(X^{\mathbb{N}}, \mathcal{A}^{\mathbb{N}}\right.$, $\mu^{\mathbb{N}}$ ) be the associated product space (see discussion around theorem 2.1.41).

The dynamical system called unilateral shift, $\sigma: X^{\mathbb{N}} \rightarrow X^{\mathbb{N}}$, is given by $\left(x_{n}\right)_{n \geq 0} \mapsto\left(x_{n+1}\right)_{n \geq 0}$. When considered together with the Bernoulli measure, the system $\left(\sigma, \mu^{\mathbb{N}}\right)$ is called unilateral Bernoulli shift.

An analogous construction with $\mathbb{Z}$ instead of $\mathbb{N}$ is possible. With $\mathbb{Z}$, the coming conclusions will still hold, added to the fact that the (bilateral) shift is invertible.

The first fact in which we are interested is that $\left(\sigma, \mu^{\mathbb{N}}\right)$ is an invariant system. By propositions 2.1.16 and 2.1.17, it is enough to check that the preimage of a cylinder is a cylinder and both have the same measure (cylinders generate the product $\sigma$-algebra, see discussion preceding proposition 2.1.41): $\sigma^{-1}\left(\left[m ; B_{m}, \ldots, B_{m+k}\right]\right)=\left[m+1 ; B_{m}, \ldots, B_{m+k}\right]$ and $\mu^{\mathbb{N}}\left(\left[m ; B_{m}, \ldots, B_{m+k}\right]\right)=$ $\mu\left(B_{m}\right) \ldots \mu\left(B_{m+k}\right)=\mu^{\mathbb{N}}\left(\left[m+1 ; B_{m}, \ldots, B_{m+k}\right]\right)$.

The second fact in which we are interested is that $\left(\sigma, \mu^{\mathbb{N}}\right)$ is an ergodic system. This is a little more demanding.

The first statement in this direction is that cylinders decouple: let $B$ and $C$ be two elements in the algebra generated by cylinders, $\mathcal{A}(\mathcal{C})$ (coinciding with the family of finite unions of disjoint cylinders, $\mathcal{D}(\mathcal{C})$, as discussed before theorem 2.1.41), then $\exists j_{0} \geq 1$ such that $\mu^{\mathbb{N}}\left(B \cap \sigma^{-j}(C)\right)=$ $\mu^{\mathbb{N}}(B) \mu^{\mathbb{N}}(C), \forall j \geq j 0$.

For now, assume $B$ and $C$ are cylinders, respectively $\left[b ; B_{b}, \ldots, B_{b+t}\right]$ and $\left[c ; C_{c}, \ldots, C_{c+s}\right]$. Define $j_{0}=b+t-c+1$. So $B$ imposes conditions up to the $(b+t)$-th entry, $C$ imposes conditions starting from the $c$-th entry and $\sigma^{-j}(C)$ imposes conditions starting from the $(c+j)$ th entry. So, when $j \geq j_{0}, c+j \geq c+j_{0}=(b+t)+1$, the conditions imposed by the cylinders $B$ and $\sigma^{-j}(C)$ are completely separate, or decoupled. In fact, $B \cap \sigma^{-j}(C)$ is a larger cylinder $\left[b ; B_{b}, \ldots, B_{b+t}, X, \ldots, X, C_{c}, \ldots, C_{c+s}\right]$, where, in the middle, the block "X,.,$X$ " has $j-(b+t)-1$ repetitions of $X$. Therefore, $\mu^{\mathbb{N}}\left(B \cap \sigma^{-j}(C)\right)=\mu\left(B_{b}\right) \cdot \ldots \cdot \mu\left(B_{b+t}\right) \cdot 1 \cdot \ldots \cdot 1 \cdot \mu\left(C_{c}\right) \cdot \ldots \cdot \mu\left(C_{c+s}\right)=$ $\mu^{\mathbb{N}}(B) \mu^{\mathbb{N}}(C)$.

Now let $B=\dot{\cup}_{u=1}^{p} B_{u}$ and $C=\dot{\cup}_{v=1}^{q} C_{v}$ be finite unions of disjoint cylinders. Rewrite the cylinders more explicitly as $B=\dot{\cup}_{u=1}^{p}\left[b_{u} ; B_{b_{u}}^{u}, \ldots B_{b_{u}+k_{b_{u}}}^{u}\right]$ and $C=\dot{\cup}_{v=1}^{q}\left[c_{v} ; C_{c_{v}}^{v}, \ldots C_{c_{v}+k_{c_{v}}}^{v}\right]$. Define $j_{1}=\max \left\{b_{1}+k_{b_{1}}, \ldots, b_{p}+k_{b_{p}}\right\}, j_{2}=\min \left\{c_{1}, \ldots, c_{q}\right\}$ and $j_{0}=j_{1}-j_{2}+1$. As such, B imposes conditions up to the $j_{1}$-th entry, $C$ imposes conditions starting from the $j_{2}$-th entry and $\sigma^{-j}(C)$ imposes conditions starting from the $\left(j_{2}+j\right)$-th entry. So, when $j \geq j_{0}, j_{2}+j \geq j_{2}+j_{0}=j_{1}+1$, the conditions imposed by $B_{u}$ and $\sigma^{-j}\left(C_{v}\right)$ (for any $1 \leq u \leq p, 1 \leq v \leq q$ ), as well as by $B$ and $\sigma^{-j}(C)$, are completely separate, or decoupled.

Finally, for $j \geq j_{0}$,

$$
\begin{gathered}
\mathbb{N}\left(B \cap \sigma^{-j}(C)\right)=\mu^{\mathbb{N}}\left(B \cap \dot{\cup}_{v=1}^{q} \sigma^{-j}\left(C_{v}\right)\right)=\mu^{\mathbb{N}}\left(\dot{\cup}_{v=1}^{q}\left(B \cap \sigma^{-j}\left(C_{v}\right)\right)\right)=\mu^{\mathbb{N}}\left(\dot{\cup}_{v=1}^{q}\left(\left(\dot{\cup}_{u=1}^{p} B_{u}\right) \cap \sigma^{-j}\left(C_{v}\right)\right)\right) \\
=\mu^{\mathbb{N}}\left(\dot{\cup}_{v=1}^{q}\left(\dot{\cup}_{u=1}^{p} B_{u} \cap \sigma^{-j}\left(C_{v}\right)\right)\right)=\mu^{\mathbb{N}}\left(\dot{\cup}_{1 \leq u \leq p, 1 \leq v \leq q} B_{u} \cap \sigma^{-j}\left(C_{v}\right)\right)=\sum_{1 \leq u \leq p, 1 \leq v \leq q} \mu^{\mathbb{N}}\left(B_{u} \cap \sigma^{-j}\left(C_{v}\right)\right) \\
\stackrel{j \leq j_{0}}{=} \sum_{1 \leq u \leq p, 1 \leq v \leq q} \mu^{\mathbb{N}}\left(B_{u}\right) \mu^{\mathbb{N}}\left(C_{v}\right)=\sum_{1 \leq v \leq q}\left(\sum_{1 \leq u \leq p} \mu^{\mathbb{N}}\left(B_{u}\right) \mu^{\mathbb{N}}\left(C_{v}\right)\right) \\
=\sum_{1 \leq v \leq q}\left(\left(\sum_{1 \leq u \leq p} \mu^{\mathbb{N}}\left(B_{u}\right)\right) \mu^{\mathbb{N}}\left(C_{v}\right)\right)=\left(\sum_{1 \leq u \leq p} \mu^{\mathbb{N}}\left(B_{u}\right)\right)\left(\sum_{1 \leq v \leq q} \mu^{\mathbb{N}}\left(C_{v}\right)\right)=\mu^{\mathbb{N}}(B) \mu^{\mathbb{N}}(C)
\end{gathered}
$$

, as desired.

Why is that useful to prove ergodicity? Because by letting $A$ be a $\sigma$-invariant element of the algebra $\left(\mu^{\mathbb{N}}\left(A \triangle \sigma^{-1}(A)\right)=0 \Rightarrow \mu^{\mathbb{N}}\left(A \cap \sigma^{-j}(A)\right)=\mu^{\mathbb{N}}(A), \forall j \geq 0\right)$ and by using $B:=A, C:=A$ in the preceding calculation, we obtain that: $\mu^{\mathbb{N}}(A)=\mu^{\mathbb{N}}\left(A \cap \sigma^{-j}(A)\right) \stackrel{(*)}{=} \mu^{\mathbb{N}}(A)^{2} \Rightarrow \mu^{\mathbb{N}}(A) \in\{0,1\}$ (in 
$(*)$, it suffices to choose a large enough $j$ ).

We still have to consider that the invariant set $A$ is in the $\sigma$-algebra (and not in the algebra). In this case, using proposition 2.1.4, we approximate it with $B$ in $\mathcal{A}=\mathcal{A}(\mathcal{C})$, the algebra generated by the cylinders: $\forall \epsilon>0, \exists B \in \mathcal{A}$ such that $\mu^{\mathbb{N}}(A \triangle B)<\epsilon$. Fix $j_{0}$ large enough to decouple $B$, i.e., implying that $\mu^{\mathbb{N}}\left(B \cap \sigma^{-j}(B)\right)=\mu^{\mathbb{N}}(B) \mu^{\mathbb{N}}\left(\sigma^{-1}(B)\right)=\mu^{\mathbb{N}}(B)^{2}, \forall j \geq j_{0}$.

We will use that $\left(A \cap \sigma^{-j}(A)\right) \triangle\left(B \cap \sigma^{-j}(B)\right) \subset(A \triangle B) \cup \sigma^{-j}(A \triangle B)$. For instance:

$$
\begin{gathered}
\left(A \cap \sigma^{-j}(A)\right) \Delta\left(B \cap \sigma^{-j}(B)\right)=\left(\left(A \cap \sigma^{-j}(A)\right) \cap\left(B \cap \sigma^{-j}(B)\right)^{\mathrm{c}}\right) \cup\left(\left(A \cap \sigma^{-j}(A)\right)^{\mathrm{c}} \cap\left(B \cap \sigma^{-j}(B)\right)\right) \\
=\left(\left(A \cap \sigma^{-j}(A)\right) \cap\left(B^{\mathrm{c}} \cup \sigma^{-j}(B)^{\mathrm{c}}\right)\right) \cup\left(\left(A^{\mathrm{c}} \cup \sigma^{-j}(A)^{\mathrm{c}}\right) \cap\left(B \cap \sigma^{-j}(B)\right)\right) \\
=\left(\left(A \cap \sigma^{-j}(A) \cap B^{\mathrm{c}}\right) \cup\left(A \cap \sigma^{-j}(A) \sigma^{-j}(B)^{\mathrm{c}}\right)\right) \cup\left(\left(A^{\mathrm{c}} \cap B \cap \sigma^{-j}(B)\right) \cup\left(\sigma^{-j}(A)^{\mathrm{c}} \cap B \cap \sigma^{-j}(B)\right)\right) \\
\subset\left(\left(A \cap B^{\mathrm{c}}\right) \cup\left(\sigma^{-j}(A) \cap \sigma^{-j}(B)^{\mathrm{c}}\right)\right) \cup\left(\left(A^{\mathrm{c}} \cap B\right) \cup\left(\sigma^{-j}(A)^{\mathrm{c}} \cap \sigma^{-j}(B)\right)\right) \\
=(A \triangle B) \cup\left(\sigma^{-j}(A) \triangle \sigma^{-j}(B)\right)=(A \triangle B) \cup \sigma^{-j}(A \triangle B)
\end{gathered}
$$

We will use that $\left|\mu^{\mathbb{N}}\left(E_{1}\right)-\mu^{\mathbb{N}}\left(E_{2}\right)\right| \leq \mu^{\mathbb{N}}\left(E_{1} \triangle E_{2}\right)$, for any $j$. As a general fact (i.e., it would work for any probability space),

$$
\begin{gathered}
\left|\mu^{\mathbb{N}}\left(E_{1}\right)-\mu^{\mathbb{N}}\left(E_{2}\right)\right|=\left|\mu^{\mathbb{N}}\left(E_{1} \backslash E_{2} \dot{\cup} E_{1} \cap E_{2}\right)-\mu^{\mathbb{N}}\left(E_{2} \backslash E_{1} \dot{\cup} E_{1} \cap E_{2}\right)\right| \\
=\left|\left(\mu^{\mathbb{N}}\left(E_{1} \backslash E_{2}\right)+\mu^{\mathbb{N}}\left(E_{1} \cap E_{2}\right)\right)-\left(\mu^{\mathbb{N}}\left(E_{2} \backslash E_{1}\right)+\mu^{\mathbb{N}}\left(E_{1} \cap E_{2}\right)\right)\right| \\
=\left|\mu^{\mathbb{N}}\left(E_{1} \backslash E_{2}\right)-\mu^{\mathbb{N}}\left(E_{2} \backslash E_{1}\right)\right| \leq\left|\mu^{\mathbb{N}}\left(E_{1} \backslash E_{2}\right)+\mu^{\mathbb{N}}\left(E_{2} \backslash E_{1}\right)\right|=\mu^{\mathbb{N}}\left(E_{1} \triangle E_{2}\right)
\end{gathered}
$$

Plugging in $E_{1}:=A \cap \sigma^{-j}(A)$ and $E_{2}:=B \cap \sigma^{-j}(B)$ :

$$
\begin{gathered}
\left|\mu^{\mathbb{N}}\left(A \cap \sigma^{-j}(A)\right)-\mu^{\mathbb{N}}\left(B \cap \sigma^{-j}(B)\right)\right| \leq \mu^{\mathbb{N}}\left(A \cap \sigma^{-j}(A) \triangle B \cap \sigma^{-j}(B)\right) \leq \mu^{\mathbb{N}}\left((A \triangle B) \cup \sigma^{-j}(A \triangle B)\right) \\
\leq \mu^{\mathbb{N}}(A \triangle B)+\mu^{\mathbb{N}}\left(\sigma^{-j}(A \triangle B)\right)=2 \mu^{\mathbb{N}}(A \triangle B) \leq 2 \epsilon .
\end{gathered}
$$

Therefore $\left|\mu^{\mathbb{N}}\left(A \cap \sigma^{-j}(A)\right)-\mu^{\mathbb{N}}\left(B \cap \sigma^{-j}(B)\right)\right| \leq 2 \epsilon$, for any $j$. When $j \geq j_{0}$, we have that $\mid \mu^{\mathbb{N}}(A \cap$ $\left.\sigma^{-j}(A)\right)-\mu^{\mathbb{N}}(B)^{2} \mid \leq 2 \epsilon$.

On the other hand, $\left|\mu^{\mathbb{N}}(A)^{2}-\mu^{\mathbb{N}}(B)^{2}\right|=\left|\left(\mu^{\mathbb{N}}(A)+\mu^{\mathbb{N}}(B)\right)\left(\mu^{\mathbb{N}}(A)-\mu^{\mathbb{N}}(B)\right)\right| \leq 2\left|\mu^{\mathbb{N}}(A)-\mu^{\mathbb{N}}(B)\right| \leq$ $2 \mu^{\mathbb{N}}(A \triangle B) \leq 2 \epsilon$ (using the above general fact again). Therefore, $\left|\mu^{\mathbb{N}}(A)^{2}-\mu^{\mathbb{N}}(B)^{2}\right| \leq 2 \epsilon$.

Combining the last two inequalities of the last two paragraphs: for $j \geq j_{0}$, we have that $\mid \mu^{\mathbb{N}}(A \cap$ $\left.\sigma^{-j}(A)\right)-\mu^{\mathbb{N}}(A)^{2} \mid \leq 4 \epsilon$. Since $\epsilon$ is arbitrary, $\mu^{\mathbb{N}}\left(A \cap \sigma^{-j}(A)\right)=\mu^{\mathbb{N}}(A)^{2}$. Due to the $\sigma$-invariance of $A, \mu^{\mathbb{N}}(A)=\mu^{\mathbb{N}}\left(A \cap \sigma^{-j}(A)\right)$, leading to $\mu^{\mathbb{N}}(A)=\mu^{\mathbb{N}}(A)^{2} \Rightarrow \mu^{\mathbb{N}}(A) \in\{0,1\}$, as desired.

Example 3.1.10 (Irrational rotation on the circle). Let $S^{1}=\mathbb{R} / \mathbb{Z}=\{[x]: x \in \mathbb{R}\}$, where $[x]=$ $\{\tilde{x} \in \mathbb{R}: x-\tilde{x} \in \mathbb{Z}\}$. For practical purposes, we refer to an element of $[x] \in S^{1}$ by indicating its only representative in $[0,1)$, a real number denoted $x(\bmod 1)=x-\lfloor x\rfloor$, where $\lfloor x\rfloor$ is the largest integer smaller than or equal to $x$.

Using this notation, a sum is defined on $S^{1}$, simply by letting $[x]+[y]=[x+y]$.

We introduce a natural bijection between $S^{1}$ and $\boldsymbol{S}=\left\{e^{i 2 \pi t}: t \in[0,1)\right\}, \operatorname{mapping}[t] \stackrel{\beta}{\mapsto} e^{i 2 \pi t(\bmod 1)}$. Considering the second space as a subset of $\mathbb{C}$ with the restricted topology, we obtain a topology on $S^{1}$, induced by $\beta$, given by $\left\{O \subset S^{1}: O=\beta^{-1}(H), H \subset S, H\right.$ open $\}$. With this topology, $S^{1}$ is a compact space.

Let $m$ be the Lesbegue measure on the real line restricted to $[0,1), m_{e}$ be the measure on $\left\{e^{i 2 \pi t}\right.$ : $t \in[0,1)\}$ given by $m_{e}(A)=m\left(\left\{t \in[0,1): e^{i 2 \pi t} \in A\right\}\right.$ and $m_{S^{1}}$ the measure induced from $m_{e}$ on $S^{1}$ through $\beta$.

Finally let $\theta \in \mathbb{R} \backslash \mathbb{Q}$ and $R_{\theta}: S^{1} \rightarrow S^{1}$ be such that $x \mapsto x+\theta(\bmod 1)$. Clearly, for $\theta^{\prime}=\theta(\bmod 1)$, $R_{\theta}=R_{\theta^{\prime}}$, i.e., $x+\theta(\bmod 1)=x+\theta^{\prime}(\bmod 1), \forall x \in S^{1}$. Then:

(i) $\left(R_{\theta}, m\right)$ is an invariant system.

(ii) Every orbit of $R_{\theta}$ is dense. 
(iii) $\left(R_{\theta}, m\right)$ is an ergodic system.

For (i), by proposition 2.1.17, it is enough to check that $R_{\theta}$ preserves the measures of left-closed right-opened intervals. Let $[a, b) \subset[0,1)$. The options for $f^{-1}([a, b))$ are three:

(a) $f^{-1}([a, b))=\left[a-\theta^{\prime}, b-\theta^{\prime}\right)$, if $\theta^{\prime}<a<b$,

(b) $f^{-1}([a, b))=\left[0, b-\theta^{\prime}\right) \cup\left[1-\left(\theta^{\prime}-a\right), 1\right)$, if $a \leq \theta^{\prime} \leq b$,

(c) $f^{-1}([a, b))=\left[1-\left(\theta^{\prime}-a\right),\left[1-\left(\theta^{\prime}-b\right)\right)\right.$, if $a<b<\theta^{\prime}$.

The measure of any of those preimages is $\left(b-\theta^{\prime}\right)-\left(a-\theta^{\prime}\right)=\left(b-\theta^{\prime}\right)+\left(1-\left(1-\theta^{\prime}+a\right)\right)=$ $\left(1-\theta^{\prime}+b\right)-\left(1-\theta^{\prime}+a\right)=b-a$, coinciding with the measure of the starting set, $[a, b)$. Therefore, $\left(R_{\theta}, m\right)$ is an invariant system.

For (ii), let $x \in S^{1}$ and note that its orbit $\left(R_{\theta}^{n}(x)\right)_{n \geq 0}$ admits a convergent subsequence, since $S^{1}$ is compact. Say $\lim _{k} R_{\theta}^{n_{k}}(x)=x_{0}$. Now let $x_{*} \in S^{1}$ and $0<\epsilon<\min \left\{x_{*}, 1-x_{*}, \theta^{\prime}\right\}$. Note that $\exists k_{1}<k_{2}$, and $0<q=n_{k_{2}}-n_{k_{1}}$, such that $y_{1}=R_{\theta}^{n_{k_{1}}}(x)$ and $y_{2}=R_{\theta}^{q}\left(y_{1}\right)=R_{\theta}^{n_{k_{2}}}(x)$ are both in the interval $\left(x_{0}-\epsilon / 2, x_{0}+\epsilon / 2\right)$. Then $d_{S^{1}}\left(y_{1}, y_{2}\right)<\epsilon$ and applying $R_{\theta}^{q}$ to $\left(y_{1}, y_{2}\right)$ we obtain juxtaposed intervals $\left(R_{\theta}^{q}\left(y_{1}\right)=y_{2}, R_{\theta}^{q}\left(y_{2}\right)\right), \ldots$. These intervals all have $\epsilon d_{S^{1}}$-distant extremes and wrap $S^{1}$. Therefore one of these extremes, an element of the $R_{\theta}$-orbit of $x$, will be $\epsilon d_{S^{1}}$-close to any point of $S^{1}$, in particular, of $x_{*}$.

For (iii), let $A \in \mathcal{A}$, with $m_{S^{1}}\left(A \triangle R_{\theta}^{-1}(A)\right)=0$, be such that $\mu(A) \in(0,1)$. By proposition 2.1.53, for some $A^{\prime} \subset A, A^{\prime} \in \mathcal{A}$, with $m_{S^{1}}\left(A^{\prime}\right)=m_{S^{1}}(A)$, we have that $\lim _{\epsilon \rightarrow 0} \frac{m_{S^{1}}((x-\epsilon, x+\epsilon) \cap A)}{m_{S^{1}}((x-\epsilon, x+\epsilon))}=1, \forall x \in A^{\prime}$. Let $B=A^{c}$, then, analogously, for some $B^{\prime} \subset B, B^{\prime} \in \mathcal{A}$, with $m_{S^{1}}\left(B^{\prime}\right)=m_{S^{1}}(B)$, we have that $\lim _{\epsilon \rightarrow 0} \frac{m_{S^{1}}((y-\epsilon, y+\epsilon) \cap B)}{m_{S^{1}}((y-\epsilon, y+\epsilon))}=1, \forall y \in B^{\prime}$. Since $A^{\prime}$ and $B^{\prime}$ have positive measure, select one element in each of them, $x_{0}$ and $y_{0}$, respectively. Fix $\delta>0$ a small number (we will estimate it conveniently later $)$. Choose $\epsilon_{0}<\theta^{\prime} / 2$ such that $\frac{m_{S^{1}}\left(\left(x_{0}-\epsilon, x_{0}+\epsilon\right) \cap A\right)}{m_{S^{1}}\left(\left(x_{0}-\epsilon, x_{0}+\epsilon\right)\right)}>1-\delta$ and $\frac{m_{S^{1}}\left(\left(y_{0}-\epsilon, y_{0}+\epsilon\right) \cap B\right)}{m_{S^{1}}\left(\left(y_{0}-\epsilon, y_{0}+\epsilon\right)\right)}>1-\delta, \forall \epsilon \leq \epsilon_{0}$. Take the intervals $I_{x}=\left(x_{0}-\epsilon_{0}, x_{0}+\epsilon_{0}\right)$ and $I_{y}=\left(y_{0}-\epsilon_{0}, y_{0}+\epsilon_{0}\right)$ and note that $m_{S^{1}}\left(A \cap I_{x}\right)>$ $(1-\delta) m_{S^{1}}\left(I_{x}\right)=(1-\delta) 2 \epsilon(I)$ and $m_{S^{1}}\left(B \cap I_{y}\right)>m_{S^{1}}\left(I_{y}\right)=(1-\delta) 2 \epsilon_{0}$ (II).

The coming argument is easier to understand by drawing some intervals. By (ii), choose $j>0$ such that $\left|R_{\theta}^{j}\left(x_{0}\right)-y_{0}\right|<\delta 2 \epsilon_{0}$. As a consequence, $m_{S^{1}}\left(R_{\theta}^{j}\left(I_{x}\right) \cap I_{y}\right)>2 \epsilon_{0}-\delta 2 \epsilon_{0}=(1-\delta) 2 \epsilon_{0}$. By (I), $m_{S^{1}}\left(R_{\theta}^{j}\left(A \cap I_{x}\right)\right)>(1-\delta) m_{S^{1}}\left(R_{\theta}^{j}\left(I_{x}\right)\right)=(1-\delta) 2 \epsilon_{0}$. It is easy to estimate that $m_{S^{1}}\left(R_{\theta}^{j}\left(A \cap I_{x}\right) \cap I_{y}\right)>$ $(1-\delta) 2 \epsilon_{0}-\delta 2 \epsilon_{0}=(1-2 \delta) \epsilon_{0}$. Similarly, $m_{S^{1}}\left(R_{\theta}^{j}\left(I_{x}\right) \cap\left(I_{y} \cap B\right)\right)>(1-2 \delta) 2 \epsilon_{0}$.

However, $R_{\theta}^{j}\left(A \cap I_{x}\right) \cap I_{y}$ and $R_{\theta}^{j}\left(I_{x}\right) \cap\left(I_{y} \cap B\right)$ are disjoint subsets of $R_{\theta}^{j}\left(I_{x}\right) \cap I_{y}$. Each of those first two sets has measure greater than $(1-2 \delta) 2 \epsilon_{0}$, while the former set has measure of at most $2 \epsilon_{0}$. If $2(1-2 \delta) 2 \epsilon_{0}>2 \epsilon_{0} \Leftrightarrow \delta<1 / 4$, we would arrive at a contradiction. Clearly, we could have started with $\delta<1 / 4$ - completing the argument.

Now we shift to a new topic. We state and prove some propositions related to the idea of ergodic decomposition, some of which (the last ones) provide a quite different point of view of Birkhoff's ergodic theorems. We prove and discuss the ergodic decomposition theorem using with Rokhlin's measure desintegration theorem, which is a measure theoretical result and whose proof is then omitted.

Let's fix some notation. Let $(X, \mathcal{A})$ be a measurable space. Then, for $K \in\{[0, \infty),[0, \infty], \mathbb{R}, \overline{\mathbb{R}}, \mathbb{C}\}$ :

(a) $\mathcal{M}(X, K)$ is the set of $K$-valued measures on $(X, \mathcal{A})$,

(b) $\mathcal{M}_{1}(X)$ is the set of probability measures on $(X, \mathcal{A})$.

Additionally, let $f: X \rightarrow X$ be a measurable dynamical system. Then:

(c) $\mathcal{M}(f)$ is the set $f$-invariant measures in $\mathcal{M}(X,[0, \infty])$,

(d) $\mathcal{M}_{1}(f)$ is the set of $f$-invariant measures in $\mathcal{M}_{1}(X)$,

(e) $\mathcal{M}^{e}(f)$ is the set of $f$-ergodic measures in $\mathcal{M}(X,[0, \infty])$,

(f) $\mathcal{M}_{1}^{e}(f)$ is the set of $f$-ergodic measures in $\mathcal{M}_{1}(X)$.

To make this notation suitable to that already introduced in section 2.2, we can append a "r" as a subscript, $\mathcal{M}_{r}(X, K), \mathcal{M}_{1, r}(X), \mathcal{M}_{r}(f), \mathcal{M}_{1, r}(f), \mathcal{M}_{r}^{e}(f)$ and $\mathcal{M}_{1, r}^{e}(f)$, to indicate that one should consider a set with analogous constraints but consisting only of Radon measures/probabilities on $\left(X, \mathcal{B}_{X}\right)$, where $X$ is $\mathrm{LCH}$.

The first proposition preceding the ergodic decomposition theorem is the following. 
Proposition 3.1.11 (Invariant measures absolutely continuous w.r.t. ergodic ones are ergodic). Let $(X, \mathcal{A})$ be a measurable space and $f: X \rightarrow X$ a measurable dynamical system. If $\nu \in \mathcal{M}_{1}(f)$, $\mu \in \mathcal{M}_{1}^{e}(f)$ and $\nu \ll \mu$, then $\nu=\mu$.

Proof. To see why, choose some $\psi \in L^{1}(\mu, \mathbb{R})$. Then, by Birkhoff's ergodic theorem with ergodicity, $\dot{Q}(\psi)(x)=\int \psi d \mu$ for all $x \in X$, except for those in a set $E$, with $\mu(E)=0$. Since $\nu \ll \mu, \nu(E)=0$. So we obtain directly that $\dot{Q}(\psi)(x)=\int \psi d \mu$ for all $x \in X$, except for those in a set $E$, with $\nu(E)=0$. Using Birkhoff's ergodic theorem for $\nu$, we also have that $\int \dot{Q}(\psi) d \nu=\int \psi d \nu$, but obviously $\int \dot{Q}(\psi) d \nu=\int \psi d \mu$. Therefore $\int \psi d \nu=\int \psi d \mu, \forall \psi \in L^{1}(\mu, \mathbb{R})$. Considering, in particular, $\psi$ 's as characteristic functions, we conclude that $\nu=\mu$.

The second proposition preceding the ergodic decomposition theorem recognizes that $\mathcal{M}_{1}(f)$ is a convex set and conclude that $\mathcal{M}_{1}^{e}(f) \subset \mathcal{M}_{1}(f)$ constitutes the set of its extreme points (i.e., those elements in a convex set that can not be written as non-trivial linear combination of other elements in it).

Theorem 3.1.12 (Ergodic measures are extreme points). Let $(X, \mathcal{A})$ be a measurable space and $f: X \rightarrow X$ a measurable dynamical system. Let $\mu \in \mathcal{M}_{1}(f)$. Then $\mu$ is ergodic if, and only if, $\mu$ is an extreme point of $\mathcal{M}_{1}(f)$ (i.e., $\nexists t \in(0,1), \nu_{1}, \nu_{2} \in \mathcal{M}_{1}(f), \nu_{1} \neq \nu_{2}$, such that $\left.\mu=t \nu_{1}+(1-t) \nu_{2}\right)$.

Proof. For $(\Rightarrow)$, suppose, for a contradiction, that $\exists t \in(0,1), \nu_{1}, \nu_{2} \in \mathcal{M}_{1}(f), \nu_{1} \neq \nu_{2}$, such that $\mu=t \nu_{1}+(1-t) \nu_{2}$. Then for every $A \in \mathcal{A}$ such that $\mu(A)=0$, it should be the case that $\nu_{1}(A)=0$ and $\nu_{2}(A)=0$, hence $\nu_{1} \ll \mu$ and $\nu_{2} \ll \mu$. Therefore, by the previous proposition, $\nu_{1}=\mu$ and $\nu_{2}=\mu$, but $\nu_{1} \neq \nu_{2}$, a contradiction.

For $(\Leftarrow)$, we take the contrapositive ( $\mu$ non ergodic $\Rightarrow \mu$ admits a non-trivial decomposition). Let be $\mu \in \mathcal{M}_{1}^{e}(f)$ and $A \in \mathcal{A}$, with $\mu\left(A \triangle f^{-1}(A)\right)=0$, such that $\mu(A) \in(0,1)$. We know that, $\forall B \in \mathcal{A}, \mu(B)=\mu(A \cap B)+\mu\left(A^{\mathrm{c}} \cap B\right)$. But $\left.\mu\right|_{A}(B)=\mu(A \cap B)$ and $\left.\mu\right|_{A^{\mathrm{c}}}(B)=\mu\left(A^{\mathrm{c}} \cap B\right)$ are measures but not probabilities and the coefficients are 1 and 1 (not summing up to 1 ). In order to adapt it, we can normalize $\left.\mu\right|_{A}$ and $\left.\mu\right|_{A^{\mathrm{c}}}$, by dividing them by $\mu(A)$ and $\mu\left(A^{\mathrm{c}}\right)$, respectively, to obtain the probability measures $\mu_{A}(B)=\mu(A \cap B) / \mu(A)$ and $\mu_{A^{c}}(B)=\mu\left(A^{c} \cap B\right) / \mu\left(A^{c}\right)$. So $\mu(B)=$ $\frac{\mu(A)}{\mu(A)} \mu(A \cap B)+\frac{\mu\left(A^{\mathrm{c}}\right)}{\mu\left(A^{\mathrm{c}}\right)} \mu\left(A^{\mathrm{c}} \cap B\right)=\mu(A) \frac{\mu(A \cap B)}{\mu(A)}+\mu\left(A^{\mathrm{c}}\right) \frac{\mu\left(A^{\mathrm{c}} \cap B\right)}{\mu\left(A^{\mathrm{c}}\right)}=\mu(A) \mu_{A}(B)+\mu\left(A^{\mathrm{c}}\right) \mu_{A^{\mathrm{c}}}(B)$. Also, it is easy to conclude $\mu_{A}$ and $\mu_{B}$ are invariant. Therefore $\mu$ admits a non-trivial decomposition.

The third and last proposition preceding the ergodic decomposition theorem has two versions. They state that a family of ergodic measures is pairwise mutually singular, with each living in disjoint $f$-invariant regions of the space.

Proposition 3.1.13 (Ergodic measures are mutually singular on $f$-invariant regions, v1). Let $(X, \mathcal{A})$ be a measurable space whose $\sigma$-algebra has a countable generator $G, \mathcal{A}=\sigma(G)$. Let $f: X \rightarrow X$ be a measurable dynamical system and $\left(\mu_{n}\right)_{n \in \mathcal{N}}$ be an arbitrary family of distinct ergodic probability measures. Then there exists $\left(P_{n}\right)_{n \in \mathcal{N}}$ in $\mathcal{A}$ such that $P_{n} \cap P_{n^{\prime}}=\varnothing, n \neq n^{\prime}, f\left(P_{n}\right)=P_{n}$ and $\mu_{n}\left(P_{n}\right)=1$, $\forall n \in \mathcal{N}$.

Proof. Let $\mathcal{A}$ be the (countable) algebra generated by $G$. Define $P_{n}=\left\{x \in X: \lim _{m} 1 / m \sum_{j=0}^{m-1} \mathcal{X}_{A}\right.$ 。 $\left.f^{j}(x)=\mu_{n}(A), \forall A \in \mathcal{A}\right\}=\bigcap_{A \in \mathcal{A}} Q\left(\mathcal{X}_{A}\right)^{-1}\left(\left\{\mu_{n}(A)\right\}\right)$, for $n \in \mathcal{N}$. A conclusion that can be drawn from theorem 3.1.3, irrespective of the choice of the invariant measure $\mu$, is that $Q\left(\mathcal{X}_{A}\right): X_{\mathcal{X}_{A}} \rightarrow \mathbb{R}$ is measurable for all $A \in \mathcal{A}$, so those preimages are measurable as well as their countable intersection, therefore $P_{n}$ is measurable for every $n \in \mathcal{N}$.

For a given $\mu_{n}$ in the family, by theorem 3.1.7, we know that, for each $A \in \mathcal{A}, Q\left(\mathcal{X}_{A}\right)=\mu_{n}(A)$ $\mu_{n}$-a.e., hence $\mu_{n}\left(Q\left(\mathcal{X}_{A}\right)^{-1}\left(\left\{\mu_{n}(A)\right\}\right)\right)=1$. Thus, as a countable intersection, $\mu_{n}\left(P_{n}\right)=1$, for all $n \in \mathcal{N}$.

The sets $P_{n}$ are pairwise disjoint because, otherwise, we would have a contradiction: $x \in P_{n} \cap$ $P_{n^{\prime}} \Rightarrow \mu_{n}(A)=\lim _{m} 1 / m \sum_{j=0}^{m-1} \mathcal{X}_{A} \circ f^{j}(x)=\mu_{n^{\prime}}(A), \forall A \in \mathcal{A} \Rightarrow \mu_{n}=\mu_{n^{\prime}}$, by Caratheodory's extesion theorem.

The sets $P_{n}$ are invariant because the Birkhoff's averages used to define them are invariant, by theorem 3.1.3. 
Proposition 3.1.14 (Ergodic measures are mutually singular on $f$-invariant regions, v2). Let $(X, \mathcal{A})$ be a measurable space (not necessarily countably generated). Let $f: X \rightarrow X$ be a measurable dynamical system and $\left(\mu_{n}\right)_{n \geq 1}$ be a countable family of distinct ergodic probability measures. Then there exists $\left(P_{n}\right)_{n \geq 1}$ such that $P_{n} \cap P_{n^{\prime}}=\varnothing, n \neq n^{\prime}, f\left(P_{n}\right)=P_{n}$ and $\mu_{n}\left(P_{n}\right)=1, \forall n \geq 1$.

Proof. Let $D=\left\{p=(i, j) \in \mathbb{N}^{2}: i, j \in \mathbb{N}, i<j\right\}=\left\{p_{1}=\left(i_{1}, j_{1}\right), \ldots, p_{k}=\left(i_{k}, j_{k}\right), \ldots\right\}$. For any pair of distinct elements in the family of measures, $\mu_{i}$ and $\mu_{j}$ (suppose, without loss of generality, that $i<j)$, there exists a unique a $k_{0} \geq 1$ such that $p_{k_{0}}=\left(i_{k_{0}}, j_{k_{0}}\right)=(i, j) \in D$. Conversely, for any $k_{1} \geq 1$, there exists a unique pair of distinct elements in the family of measures, namely $\mu_{i_{k_{1}}}$ and $\mu_{j_{k_{1}}}$, where $p_{k_{1}}=\left(i_{k_{1}}, j_{k_{1}}\right)$. So we can refer interchangeably to pairs of distinct elements in the family of measures and to $p_{k}$ 's in $D$ (or to their index $k$ ).

Now for each $k \geq 1$, there exists a measurable $\psi_{k}: X \rightarrow \mathbb{R}$ such that $\int \psi_{k} d \mu_{i_{k}} \neq \int \psi_{k} d \mu_{j_{k}}$ (in particular, one can choose $\psi_{k}=\mathcal{X}_{A}$ to be a characteristic function of a measurable set once $\mu_{i_{k}}$ and $\mu_{j_{k}}$ are distinct measures).

For each $n \geq 1$ and $k \geq 1$, form the pair $\left(\mu_{n}, \psi_{k}\right)$ and, using these measure and observable, name the set arising from theorem 3.1.7 as $X_{n, k}$, i.e., $\lim _{m} 1 / m \sum_{j=0}^{m-1} \psi_{k} \circ f^{j}(x)=\int \psi_{k} d \mu_{n}, \forall x \in X_{n, k}$, with $\mu_{n}\left(X_{n, k}\right)=1$. Now let $P_{n}=\cap_{k} X_{n, k}$. Clearly, $\mu_{n}\left(P_{n}\right)=1$

The sets $P_{n}$ are pairwise disjoint because, otherwise, we would have a contradiction: $x \in P_{n} \cap$ $P_{n^{\prime}}\left(n \neq n^{\prime}\right.$, say $\left.n<n^{\prime}\right) \Rightarrow \int \psi_{k} d \mu_{n}=\lim _{m} 1 / m \sum_{j=0}^{m-1} \psi_{k} \circ f^{j}(x)=\int \psi_{k} d \mu_{n^{\prime}}, \forall k \geq 1$, but there exists some $k_{0} \geq 1$ such that $p_{k_{0}}=\left(i_{k_{0}}, j_{k_{0}}\right)=\left(n, n^{\prime}\right) \in D$ and an associated $\psi_{k_{0}}$ such that $\int \psi_{k_{0}} d \mu_{n}=$ $\int \psi_{k_{0}} d \mu_{i_{k_{0}}} \neq \int \psi_{k_{0}} d \mu_{j_{k_{0}}}=\int \psi_{k_{0}} d \mu_{n^{\prime}}$.

The sets $P_{n}$ are invariant because $x \in P_{n} \Leftrightarrow x \in X_{n, k}, \forall k \geq 1 \Leftrightarrow \lim _{m} 1 / m \sum_{j=0}^{m-1} \psi_{k} \circ f^{j}(x)=$ $\int \psi_{k} d \mu_{n}, \forall k \geq 1$, and this average, as we know from theorem 3.1.3, is $f$-invariant.

We now focus on the ergodic decomposition theorem itself. First, we state Rokhlin desintegration theorem and some preliminary definitions and propositions.

Definition 3.1.15 (Measurable partition). Let $(X, \mathcal{A}, \mu)$ be measure space. An arbitrary family $\mathcal{P}=\left\{P_{i} \in \mathcal{A}: i \in \mathcal{I}\right\}$ is a measurable partition of $(X, \mathcal{A}, \mu)$ if there exists $X_{0} \in \mathcal{A}, X_{0} \subset X, \mu\left(X_{0}^{\mathrm{c}}\right)=0$, such that:

(i) $\mathcal{P} \cap X_{0}=\left\{P_{i} \cap X_{0}: i \in \mathcal{I}\right\}$ is a partition of $X_{0}$, i.e., $\bigcup_{i \in \mathcal{I}} P_{i} \cap X_{0}=X_{0}$ and $\left(P_{i} \cap X_{0}\right) \cap\left(P_{j} \cap X_{0}\right)=$ $\varnothing\left(\Leftrightarrow P_{i} \cap P_{j}=\varnothing\right), \forall i, j \in \mathcal{I}, i \neq j$.

(ii) there exists a sequence of partitions of $X_{0},\left(\mathcal{P}_{n}\right)_{n \geq 0}$, with each $\mathcal{P}_{n}$ being countable and composed of measurable sets, such that (a) $\mathcal{P}_{0}<\mathcal{P}_{1}<\ldots$ and (b) $\mathcal{P} \cap X_{0}=\bigvee_{n \geq 0} \mathcal{P}_{n}$. Here, $\mathcal{P}_{n}<P_{n+1}$ means every element of $\mathcal{P}_{n+1}$ is contained in an element of $\mathcal{P}_{n}$ and $\mathcal{V}=\bigvee_{n \geq 0} \mathcal{P}_{n}$ is the partition of $X_{0}$ given by $\mathcal{V}=\left\{P=\cap_{n \geq 0} P_{n} \in \mathcal{A}: P_{n} \in \mathcal{P}_{n}, \forall n \geq 0\right.$, such that $\left.\cap_{n \geq 0} P_{n} \neq \varnothing\right\}$.

Note that $x \in X_{0} \Rightarrow \exists ! P_{n}(x) \in \mathcal{P}_{n}$ such that $x \in P_{n}(x) \Rightarrow P_{0}(x) \supset P_{1}(x) \supset \ldots \Rightarrow P(x)=$ $\cap_{n \geq 0} P_{n}(x) \neq \varnothing$ is in $\mathcal{V}$ and $x \in P(x)$, hence $X_{0} \subset \cup_{P \in \mathcal{V}} P$. Conversely, $x \in \cup_{P \in \mathcal{V}} P \Rightarrow x \in P_{i_{0}}$, for some $P_{i_{0}} \in \mathcal{V} \Rightarrow \exists P_{n} \in \mathcal{P}_{n}, \forall n \geq 0$, such that $x \in P_{i_{0}}=\cap_{n \geq 0} P_{n} \Rightarrow x \in X_{0}$, hence $\cup_{P \in \mathcal{V}} P \subset X_{0}$. Therefore $\cup_{P \in \mathcal{V}} P=X_{0}$.

To check that elements of $\mathcal{V}$ are disjoint, let $P, P^{\prime} \in \mathcal{V}$ be such that $P \neq P^{\prime}$ and $P, P^{\prime} \neq \varnothing$. Then $P=\cap_{n \geq 0} P_{n}$, for some $P_{n} \in \mathcal{P}_{n}, n \geq 0$, and $P^{\prime}=\cap_{n \geq 0} P_{n}^{\prime}$, for some $P_{n}^{\prime} \in \mathcal{P}_{n}, n \geq 0$. Therefore $x \in P \Rightarrow x \in P_{n}, \forall n \geq 0 \Rightarrow x \notin P_{n}^{\prime}, \forall n \geq 0 \Rightarrow x \notin P^{\prime} \Rightarrow P \cap P^{\prime}=\varnothing$.

That is, $\mathcal{V}$, as defined, is really a partition of $X_{0}$ whose elements are measurable sets. Moreover, its definition implies that $\mathcal{P}_{n}<\mathcal{V}, \forall n \geq 0\left(P \in \mathcal{V}, P \neq \varnothing \Rightarrow P=\cap_{n \geq 0} P_{n}, P_{n} \in \mathcal{P}_{n} \Rightarrow P \subset P_{n} \in \mathcal{P}_{n}, \forall n \geq\right.$ $0)$, and that there is no other partition $\mathcal{U}$ of $X_{0}$ whose elements are measurable sets such that $\mathcal{P}_{n}<$ $\mathcal{U}, \forall n \geq 0$ and $\mathcal{U}<\mathcal{V}$. For such, suppose there exists one such $\mathcal{U} \neq \mathcal{V}, \mathcal{P}_{n}<\mathcal{U}<\mathcal{V}, \forall n \geq 0 \Rightarrow \exists x \in X_{0}$ such that $P(x) \in \mathcal{V}$ and $Q(x) \in \mathcal{U}$, the unique elements of each partition containing $x$, are different $\Rightarrow P(x) \varsubsetneqq Q(x) \Rightarrow \exists y \in Q(x), y \notin P(x)=\cap_{n \geq 0} P_{n}(x), P_{n}(x) \in \mathcal{P}_{n}, n \geq 0 \Rightarrow y \notin P_{n_{0}}(x)$ for some $n_{0} \geq 0$. But, since $\mathcal{P}_{n_{0}}<\mathcal{U}, Q(x) \mp P_{n_{0}}(x)$, leading to a contradiction.

Clearly, any finite or countable partition $\mathcal{P}$ of a subset $X_{0} \subset X, X_{0} \in \mathcal{A}$, with $\mu\left(X_{0}^{\mathrm{c}}\right)=0$, whose elements are measurable sets, is a measurable partition (choose $\mathcal{P}_{n}=\mathcal{P}, \forall n \geq 0$ ). But, differently from its approximating partitions $\mathcal{P}_{n}$ 's, a measurable partition $\mathcal{P}$ can be uncountable. For example, let 
$X=[0,1) \times[0,1), \mathcal{A}=\mathcal{B}_{X}, \mu=$ Lesbegue on $\mathbb{R}^{2}$ restricted to $X, \mathcal{P}=\{\{x\} \times[0,1): x \in[0,1)\}, X_{0}=X$ and $\mathcal{P}_{n}=\left\{\left[j 2^{-n},(j+1) 2^{-n}\right) \times[0,1): j=0, \ldots, 2^{n}-1\right\}$.

On the other hand, not every partition is measurable. Let $R_{\theta}: S^{1} \rightarrow S^{1}$ be an irrational rotation (a homeomorphism), $X=S^{1}, \mathcal{A}=\mathcal{B}_{X}, \mu=$ Lesbegue probability induced on $S^{1}$ and $\mathcal{P}=\left\{\left\{R_{\theta}^{n}(x)\right.\right.$ : $\left.n \in \mathbb{Z}\}: x \in S^{1}\right\}$ be the partition induced from orbits. If $\mathcal{P}$ was a measurable partition, there would exist $X_{0} \subset X, X_{0} \in \mathcal{A}, \mu\left(X_{0}\right)=1$, and $\mathcal{P}_{0}<\mathcal{P}_{1}<\ldots$, such that, in particular, for any $x \in X_{0},\left\{R_{\theta}^{n}(x)\right.$ : $n \in \mathbb{Z}\} \cap X_{0}=\cap_{n \geq 0} P_{n}(x)$. We want to argue that $\mu$-a.e. $x \in X_{0}$ has its orbit completely inside $X_{0}$. Otherwise, there would exist a set $X_{0}^{\prime} \subset X_{0}, \mu\left(X_{0}^{\prime}\right)>0$, such that $\left\{R_{\theta}^{n}(x): n \in \mathbb{Z}\right\} \cap X_{0}^{c} \neq \varnothing, \forall x \in X_{0}^{\prime}$. So $X_{0}^{\prime} \subset \cup_{n \in \mathbb{Z}} R_{\theta}^{n}\left(X_{0}^{\mathrm{c}}\right)$, but $\mu\left(X_{0}^{\prime}\right)>0$ and $\mu\left(\cup_{n \in \mathbb{Z}} R_{\theta}^{n}\left(X_{0}^{\mathrm{c}}\right)\right)=0$, a contradiction. So there exists $X_{0}^{*} \subset X_{0}, \mu\left(X_{0}^{*}\right)=1$, such that: $\forall x \in X_{0}^{*},\left\{R_{\theta}^{n}(x): n \in \mathbb{Z}\right\} \subset X_{0} \Rightarrow \forall x \in X_{0}^{*},\left\{R_{\theta}^{n}(x): n \in \mathbb{Z}\right\} \subset P_{n}(x)$. Therefore, each of the $P_{n}(x)$ 's, for $x \in X_{0}^{*}$, is such that $\mu\left(P_{n}(x) \triangle R_{\theta}^{-1}\left(P_{n}(x)\right)\right)=0$ (because it contains the full orbit of most of its points), implying, by ergodicity, $\mu\left(P_{n}(x)\right) \in\{0,1\}, \forall x \in X_{0}^{*}, n \geq$ 0 . Thus, for a fixed $n$, there can be no distinct sets $P_{n}(x)$ and $P_{n}\left(x^{\prime}\right), x, x^{\prime} \in X_{0}^{*}$, having measure 1 , but it also can not happen that $\mu\left(P_{n}(x)\right)=0, \forall x \in X_{0}^{*}$, since $X \backslash \cup_{x \in X_{0}^{*}} P_{n}(x)$ (which boils down to a countable union) is a subset of $X_{0}^{* c}$, whose measure is 0 . In conclusion, for each $n \geq 0$, there should be exactly one set of the form $P_{n}\left(x_{n}\right) \in \mathcal{P}_{n}, x_{n} \in X_{0}^{*}$, with $\mu\left(P_{n}\left(x_{n}\right)\right)=1$. Now take $P^{*}=\cap_{n \geq 0} P_{n}\left(x_{n}\right), \mu\left(P^{*}\right)=1$. But $P^{*} \in \mathcal{P}$, so we found a set of an orbit having full Lebesgue measure - a contradiction. Therefore, $\mathcal{P}$ can not be a measurable partition.

Now we are used with the idea of a measurable partition, we state Rokhlin's desintegration theorem.

Theorem 3.1.16 (Rokhlin's desintegration theorem). Let $M$ be a complete separable metric space, $\left(M, \mathcal{B}_{M}, \mu\right)$ be a Borelian probability space and $\mathcal{P}$ be a measurable partition of $\left(M, \mathcal{B}_{M}, \mu\right)$ (associated to the set of full measure $\left.M_{0}\right)$. So there exists a family $\left\{\mu_{P}: P \in \mathcal{P}\right\}$ of probabilities on $\left(M, \mathcal{B}_{M}\right)$, called a desintegration of $\mu$ with respect to $\mathcal{P}$, satisfying:

(i) $\mu_{P}(P)=1$, $\hat{\mu}$-a.e.P $\in \mathcal{P}$,

(ii) the mapping $P \in \mathcal{P} \mapsto \mu_{P}(E) \in \mathbb{R}$ is measurable, $\forall E \in \mathcal{B}_{M}$,

(iii) $\mu(E)=\int \mu_{P}(E) d \hat{\mu}(P), \forall E \in \mathcal{B}_{M}$,

where, using $\pi_{0}: M_{0} \rightarrow \mathcal{P}$ given by $x \mapsto \pi_{0}(x)=P(x) \in \mathcal{P}$, the only element of $\mathcal{P}$ containing $x$, the probability space $(\mathcal{P}, \hat{\mathcal{B}}, \hat{\mu})$ is defined as: (a) $\mathcal{Q} \in \hat{\mathcal{B}} \Leftrightarrow \pi_{0}{ }^{-1}(\mathcal{Q})=\cup_{P \in \mathcal{Q}} P \in \mathcal{B}_{M}$ (it is easy to check this defines a $\sigma$-algebra), (b) $\forall \mathcal{Q} \in \hat{\mathcal{B}}, \hat{\mu}(\mathcal{Q})=\mu\left(\pi_{0}^{-1}(\mathcal{Q})\right.$ ) (it is easy to check this defines a probability).

Additionally, let $(X, \mathcal{A}, \mu)$ be a probability space whose $\sigma$-algebra admits a countable generator $\mathcal{G}$ (a particular case is when $X=M$ is a complete separable metric space), $\mathcal{A}=\sigma(\mathcal{G})$, and $\mathcal{P}$ be a measurable partition of $(X, \mathcal{A}, \mu)$ such that $\mu$ admits two desintegrations with respect to $\mathcal{P}$, i.e., two families $\left\{\mu_{P}: P \in \mathcal{P}\right\}$ and $\left\{\mu_{P}^{\prime}: P \in \mathcal{P}\right\}$ of probabilities on $(X, \mathcal{A})$ satisfying (i), (ii) and (iii). Then $\mu_{P}=\mu_{P}^{\prime}$, $\hat{\mu}$-a.e.P $\in \mathcal{P}$.

The ergodic decomposition theorem follows from the previous theorem. We will omit this proof here. In addition to [VO16], the reader is invited to consider additional sources on this proof technique, such as [Tao], [Hoc] and [Mor].

Theorem 3.1.17 (Ergodic decomposition theorem). Let $M$ be a complete separable metric space, $\left(M, \mathcal{B}_{M}\right)$ be a Borelian measure space, $f: M \rightarrow M$ a measurable dynamical system and $\mu$ a probability on $\left(M, \mathcal{B}_{M}\right)$ such that $(f, \mu)$ is an invariant system. Then there exists a measurable partition $\mathcal{P}$ of $\left(M, \mathcal{B}_{M}, \mu\right)$ (associated to the set of full measure $M_{0}$ ) and a family of probability measures $\left\{\mu_{P}: P \in \mathcal{P}\right\}$, together called an ergodic decomposition of $\mu$, satisfying:

(i) $\mu_{P}(P)=1$, $\hat{\mu}$-a.e.P $\in \mathcal{P}$,

(ii) the mapping $P \in \mathcal{P} \mapsto \mu_{P}(E) \in \mathbb{R}$ is measurable, $\forall E \in \mathcal{B}_{M}$,

(iii) $\left(f, \mu_{P}\right)$ is an ergodic system, $\hat{\mu}$-a.e.P $\in \mathcal{P}$,

(iv) $\mu(E)=\int \mu_{P}(E) d \hat{\mu}(P), \forall E \in \mathcal{B}_{M}$,

where the probability space $(\mathcal{P}, \hat{\mathcal{B}}, \hat{\mu})$ is defined as in theorem 3.1.16. 
This theorem shows that under adequate hypothesis, every invariant measure can be expressed as a sort of (uncountable) convex linear combination of ergodic measures, which were also described as extreme points of the space of invariant Borelian probabilities.

Applying the previous theorem, like we did in theorem 3.1.8, but using no ergodicity, we will characterize the measure $\lambda_{x}$ presented in theorem 3.1.4, thereby refining its conclusions. As an exception, the following result was not from [VO16] and, as long as the author is aware, it is original.

Theorem 3.1.18 (Birkhoff's ergodic theorem, v2'). Let $\bar{M}$ be a compact metric space, $\left(\bar{M}, \mathcal{B}_{\bar{M}}\right)$ be a Borelian measurable space, $f: \bar{M} \rightarrow \bar{M}$ be a measurable dynamical system and $\mu$ be a probability on $\left(\bar{M}, \mathcal{B}_{\bar{M}}\right)$ such that $(f, \mu)$ is an invariant system.

Then $\exists M_{* *}, M_{* *} \in \mathcal{B}_{M}, \mu\left(M_{* *}\right)=1$ such that $\mu$-a.e. $x \in \bar{M}$, i.e., for all $x \in M_{* *}$ :

(i) there exists an $f$-ergodic probability $\mu_{x}$ on $\left(\bar{M}, \mathcal{B}_{\bar{M}}\right)$ such that $\alpha_{f, n}(x) \stackrel{w *}{\longrightarrow} \lambda_{x}=\mu_{x}$, or $A(f, x)=\left\{\mu_{x}\right\}$,

(ii) furthermore, there exists $P(x) \in \mathcal{B}_{\bar{M}}$ such that $x \in P(x), \mu_{x}(P(x))=1$ and $\nu(P(x))=0$ for any other ergodic probability.

Proof. Let $\mathcal{P}$ be the measurable partition of $\left(M, \mathcal{B}_{M}, \mu\right)$ (associated to the set $M_{0}$, with $\left.\mu\left(M_{0}\right)=1\right)$ and $\left\{\mu_{P}: P \in \mathcal{P}\right\}$ be the family of probability measures, forming an ergodic decomposition, exactly as obtained from theorem 3.1.17. For any $x \in M_{0}$, denote $P(x)$ the single element of $\mathcal{P}$ containing $x$. Also, let $\mathcal{Q} \subset \mathcal{P}, \mathcal{Q} \in \hat{\mathcal{B}}, \hat{\mu}(\mathcal{Q})=1$, be the set in which all $\hat{\mu}$-a.e. properties in theorem 3.1.17 hold, in particular, such that $\mu_{P}$ is ergodic, $\forall P \in \mathcal{Q}$. Hence $M_{1}=\pi_{0}^{-1}(\mathcal{Q})$ is such that $\mu\left(M_{1}\right)=1$.

Now consider the set $M_{\star}$ obtained in the theorem 3.1.4, $M_{0}$ and $M_{1}$ as presented above. Let $M_{* *}=M_{0} \cap M_{1} \cap M_{*}$. Clearly, $\mu\left(M_{* *}\right)=1$.

Now fix an arbitrary $x \in M_{* *}$. Since $x \in M_{*}$, the associated functional $\lambda_{x}$ is a probability measure such that $\alpha(f, x)_{n} \stackrel{w *}{\longrightarrow} \lambda_{x}$. Since $x \in M_{0}, x \in P(x)$, and we know, from the proof of theorem 3.1.17, that $\lim _{n} 1 / n \sum_{j=0}^{n-1} \psi \circ f^{j}(x)=\int \psi d \mu_{P(x)}, \forall \psi \in L^{1}\left(\mu_{P(x)}, \mathbb{R}\right)$, in particular $\alpha_{f, n}(x) \stackrel{w *}{\longrightarrow} \mu_{P(x)}=: \mu_{x}$. By the uniqueness of convergence in the weak $*$ start topology (which is metrizable), it follows that $\lambda_{x}=\mu_{x}$. Since $x \in M_{1}, \exists ! P \in \mathcal{Q}$ such that $x \in P$, namely $P=P(x)$, hence $\mu_{P(x)}(P(x))=1$ and $\mu_{P(x)}=\mu_{x}$ is an ergodic probability. Finally, once $\mu\left(M_{* *}\right)=1$, the desired conclusion follows.

\subsection{Birkhoff Ergodic Theorem Topological Analogue}

The present section is based on [Win10], until example 3.2.3, unless otherwise stated. The discussion following that example reflects the tastes of the author.

The following theorem is to be understood as a topological analogue of theorem 3.1.8. Both assume a compact metric ambient space, but the next theorem assumes a continuous, instead of measurable, dynamical system and the existence of a transitive and recurrent point instead of ergodicity. In fact, the next theorem has no invariant measure $\mu$ whatsoever.

Note that in the above setting, with $X$ a compact metric ambient space (thus a separable Baire space), the existence of a transitive and recurrent point is equivalent to topological irreducibility, mostly due to proposition 2.5.7. For instance, under those hypothesis, transitivity implies topological irreducibility. Conversely, if $X$ has no isolated points, topological irreducibility implies there exists a transitive and (due to proposition 2.5.8) recurrent point. Otherwise, if $X$ had isolated points, using topological irreducibility for an open singleton $\left\{x_{1}\right\}$, we would obtain that the isolated $x_{1}$ is periodic and with a dense orbit in $X$. Therefore $X$ is finite and $x_{1}$ transitive and recurrent.

All to argue that the hypothesis employed, transitivity and recurrence, is, all in all, topological irreducibility, which corresponds to ergodicity, once we understand it as measure theoretical irreducibility. Between them, no general implications can be easily traced, as they are concepts from self-sufficient theories, topological dynamics and ergodic theory, respectively. However, they can be understood as parallel irreducibility concepts, each in its domain.

To remember, as discussed in propositions 2.5.8 and 2.5.9, under pretty standard conditions (to which the next theorem is not restricted), transitivity implies recurrence. 
Finally, the analogy in the conclusion is that both describe $A(f, x)$, for $x$ in a (topologically and measure theoretical, respectively) large set.

In theorem 3.1.8, $A(f, x)=\{\mu\}$, for $\mu$-a.e. $x \in X$.

In the next theorem, the $\alpha_{f, n}(x)$ 's will not converge and $A(f, x)$ will not be a singleton, but a limiting description is still available: for $\tau$-a.a. $x \in X, \alpha_{f, n}(x)$ 's will accumulate wherever $\alpha_{f}(z)_{n}$ 's, starting from transitive points $z$, accumulate (that "is a lot"). Hence, in the characterization of $A(f, x),\{\mu\}$ becomes a set denoted $A_{0}(f)$, comprised of those measures accumulated by $\alpha_{f, n}(z)$ 's for transitive $z$ 's (all of which are invariant, as any accumulation point of $\alpha_{f, n}(x)$ 's).

Theorem 3.2.1 (Birkhoff's ergodic theorem with ergodicity, v2 - topological analogue). Let $\bar{M}$ be a compact metric space and $f: \bar{M} \rightarrow \bar{M}$ be a continuous dynamical system such that there exists a transitive and recurrent point $z_{0} \in \bar{M}$. Denote $A(f, x)=\left\{\nu\right.$ Borelian probability: $\alpha_{f, n}(x)=$ $1 / n \sum_{j=0}^{n-1} \delta_{f^{j}(x)}$ accumulates in $\nu$ on the weak* topology $\}$ and $A_{0}(f)=\bigcup_{z \text { transitive }} A(f, z)$.

Then $A(f, x)=A_{0}(f), \tau$-a.a. $x \in X$ (i.e., for all $x \in \bar{M}_{\star}$, a comeager set).

Remark. Let's translate the conclusion about accumulation of probabilities to accumulation of Birkhoff averages.

Let $z$ be any transitive point and $\alpha_{f}(z)_{n}$ accumulate on $\nu$, along the subsequence $n_{k}$ : $\alpha_{f}(z)_{n_{k}} \stackrel{w *}{\longrightarrow} \nu \Leftrightarrow\left(1 / n_{k} \sum_{j=0}^{n_{k}-1} \psi \circ f^{j}(z) \rightarrow \int \psi d \nu, \forall \psi \in C(\bar{M}, \mathbb{R})\right)$.

Then, the theorem guarantees that, $\forall x \in \bar{M}_{\star}, \alpha_{f, n}(x)$ also accumulates on $\nu$, along a subsequence $m_{k}$ :

$\alpha_{f}(x)_{m_{k}} \stackrel{w *}{\longrightarrow} \nu \Leftrightarrow\left(1 / m_{k} \sum_{j=0}^{m_{k}-1} \psi \circ f^{j}(x) \rightarrow \int \psi d \nu, \forall \psi \in C(\bar{M}, \mathbb{R})\right)$.

Proof. Consider $\mathcal{M}_{1}(\bar{M})$, the set of Borelian probability measures, equipped with the (metrizable) weak* topology, with an associated metric $d$. Define, for $n \geq 1$, the sequence of functions $\alpha_{f, n}: \bar{M} \rightarrow$ $\mathcal{M}_{1}(\bar{M})$ given by $\alpha_{f, n}(x)=1 / n \sum_{j=0}^{n-1} \delta_{f^{j}(x)}$.

We will adopt a dual notation. On one hand, $A(f, x)=\left\{\nu\right.$ Borelian probability: $\left(\alpha_{f, n}(x)\right)_{n \geq 1}$ accumulates in $\nu$ on the weak* topology identifies the set of probabilities $\nu$ accumulated when starting from $x$.

On the other hand, $X(f, \nu)=\left\{x \in \bar{M}:\left(\alpha_{f, n}(x)\right)_{n \geq 1}\right.$ accumulates in $\nu$ on the weak $*$ topology $\} \stackrel{*}{=}$ $\bigcap_{N, k \geq 1} \bigcup_{n \geq N} \alpha_{f, n}{ }^{-1}(B(\nu, 1 / k+1))$ (where $B(a, b)$ is a ball with center $a$ and radius $b$ with respect to $d$ ) identifies the set of starting points $x$ from which $\nu$ is accumulated. Equality $(*)$ holds in general. For the present purposes, this fact is technical, so it is shown separately in a lemma, presented immediately after this proof.

Of course, $\nu \in A(f, x) \Leftrightarrow x \in X(f, \nu)$.

Since there exists a transitive point $z_{0}$, the set $A\left(f, z_{0}\right)$ is non-empty. This is because the sequence $\alpha\left(f, z_{0}\right)_{n}$ is in $\mathcal{M}_{1}(\bar{M})$, a metrizable compact space, thereby admitting a convergence subsequence. Therefore, $A_{0}(f)$ is non-empty, so consider $\eta \in A_{0}(f) \subset \mathcal{M}(\bar{M})$.

Our first objective (I) is to show that $X(f, \eta)=\bigcap_{N, k \geq 1} \bigcup_{n \geq N} \alpha_{f, n}{ }^{-1}(B(\eta, 1 / k+1))$ is comeager ( $\eta$ in $A_{0}(f)$ is arbitrary). For such, we argue that each element in the intersection is (A) open and (B) dense.

Property (A) is easy. Using continuity of the $\alpha_{f, n}$ 's, $n \geq 1$, each preimage $\alpha_{f, n}{ }^{-1}(B(\eta, 1 / k+1))$ is open, as well as their union. For continuity, consider a convergent sequence $\left(x_{k}\right)_{k \geq 0}$ in $\bar{M}$ approaching $x_{*}$. For any $\psi \in C(\bar{M}, \mathbb{R}), \int \psi d \alpha_{f, n}\left(x_{k}\right)=1 / n \sum_{j=0}^{n-1} \psi\left(f^{j}\left(x_{k}\right)\right) \stackrel{k}{\rightarrow} 1 / n \sum_{j=0}^{n-1} \psi\left(f^{j}\left(x_{*}\right)\right)=\int \psi d \alpha_{f, n}\left(x_{*}\right)$, hence $\alpha_{f, n}\left(x_{k}\right)$ converges to $\alpha_{f, n}\left(x_{*}\right)$ in the weak $*$ topology as $k$ increases. Since the domain of the $\alpha_{f, n}$ 's is metrizable, this characterization guarantees that the $\alpha_{f, n}$ 's are continuous.

Property (B) is more elaborate. We break it in two steps:

(B1) show that, (i) for any $x \in \bar{M}, \nu \in A(f, x) \Rightarrow \nu \in A\left(f, f^{\tilde{n}}(x)\right), \forall \tilde{n} \geq 1$, or equivalently (ii) for any $n u \in \mathcal{M}_{1}(\bar{M}), x \in X(f, \nu) \Rightarrow f^{\tilde{n}}(x) \in X(f, \nu), \forall \tilde{n} \geq 1$, so, $f^{\tilde{n}}(x) \in \bigcup_{n \geq N} \alpha_{f, n}{ }^{-1}(B(\nu, 1 / k+1)), \forall \tilde{n}, N, k \geq$ 
(B2) considering $\eta \in A_{0}(f)$ in (B1), it follows that $\eta \in A(f, z)$ for some transitive $z$ hence, for any $N, k \geq 1$, (B1) guarantees that: $f^{\tilde{n}}(z) \in \bigcup_{n \geq N} \alpha_{f, n}{ }^{-1}(B(\nu, 1 / k+1)), \forall \tilde{n} \geq 0$. In particular $\left\{f^{\tilde{n}}(z): \tilde{n} \geq 0\right\} \subset \underset{n \geq N}{\bigcup} \alpha_{f, n}{ }^{-1}(B(\nu, 1 / k+1))$. Since the former set is dense, so is the second, as desired.

Arguments are still lacking for claim (B1). For instance, $\nu \in A(f, x) \Rightarrow \exists\left(n_{q}\right)_{q \geq 1}, n_{q} \nearrow \infty$, such that $1 / n_{q} \sum_{j=0}^{n_{q}-1} \delta_{f^{j}(x)} \stackrel{w *}{\longrightarrow} \nu$, i.e., $1 / n_{q} \sum_{j=0}^{n_{q}-1} \psi \circ f^{j}(x) \rightarrow \int \psi d \nu, \forall \psi \in C(\bar{M}, \mathbb{R})$. Now, for each $\tilde{n} \geq 1$, consider $n_{k^{*}}$ the first $n_{k} \geq \tilde{n}$ and let $m_{q}=n_{k^{*}+q}-\tilde{n}, \forall q \geq 1$. For any $\psi \in C(\bar{M}, \mathbb{R})$ :

$$
\begin{gathered}
\lim _{q} 1 / m_{q} \sum_{j=0}^{m_{q}-1} \psi \circ f^{j}\left(f^{\tilde{n}}(x)\right)=\lim _{q} 1 / m_{q} \sum_{j=0}^{m_{q}-1} \psi \circ f^{j+\tilde{n}}(x) \stackrel{l=j+\tilde{n}}{=} \\
\lim _{q}\left[\frac{1}{\left(n_{k^{*}+q}-\tilde{n}\right)} \sum_{l=\tilde{n}}^{n_{k^{*}+q}-1} \psi \circ f^{l}(x)+\frac{1}{\left(n_{k^{*}+q}-\tilde{n}\right)} \sum_{l=0}^{\tilde{n}-1} \psi \circ f^{l}(x)-\frac{1}{\left(n_{k^{*}+q}-\tilde{n}\right)} \sum_{l=0}^{\tilde{n}-1} \psi \circ f^{l}(x)\right] \\
=\lim _{q} \frac{1}{\left(n_{k^{*}+q}-\tilde{n}\right)} \sum_{l=0}^{n_{k^{*}+q}-1} \psi \circ f^{l}(x),
\end{gathered}
$$

by combining the first two sums and letting the last one go to 0 as $q \rightarrow \infty$

$$
\begin{gathered}
=\lim _{q} \frac{1}{n_{k^{*}+q}} \frac{n_{k^{*}+q}}{\left(n_{k^{*}+q}-\tilde{n}\right)} \sum_{l=0}^{n_{k^{*}+q}-1} \psi \circ f^{l}(x)=\lim _{q} \frac{1}{n_{k^{*}+q}} \sum_{l=0}^{n_{k^{*}+q}-1} \psi \circ f^{l}(x) \\
=\lim _{p} \frac{1}{n_{p}} \sum_{l=0}^{n_{p}-1} \psi \circ f^{l}(x)=\int \psi d \nu .
\end{gathered}
$$

It follows, as desired, that $\nu \in A\left(f, f^{\tilde{n}}(x)\right), \forall \tilde{n} \geq 1$.

So, for now, we showed (B2), (B1), (B), (A) and ultimately (I): $X(f, \eta)$ is comeager $\forall \eta \in A_{0}(f)$.

Our objective (II) is to come up with the set $\bar{M}_{\star}$ pointed out in the conclusion of the theorem. Note that, as a basic fact, if $x \in X(f, \nu)$ then $\nu \in A(f, x)$. We wish every $\eta \in A_{0}(f)$ was in $A(f, x)$ for many ( $\tau$-a.a.) $x$ 's. That is the case when, for many $x$ 's: $x \in X(f, \eta), \forall \eta \in A_{0}(f)[\diamond]$. However, this a too strong initial requirement. We use (I) and start asking $[\diamond]$ only for "sufficiently many $\eta$ 's" (a countable dense set of those). Since $\mathcal{M}_{1}(\bar{M})$ is a compact metric space, $A_{0}(f) \subset \mathcal{M}_{1}(\bar{M})$ admits a countable dense subset $\left\{\eta_{p}: p \geq 1\right\}$, so we can consider $\bar{M}_{1}=\cap_{p \geq 1} X\left(f, \eta_{p}\right)$. Clearly, $\bar{M}_{1}$ is comeager and $x \in \bar{M}_{1} \Rightarrow \eta_{p} \in A(f, x)$. Since $A(f, x)$ is closed (as a set of accumulation points of a sequence in a metric space), we have that, for all $x \in \bar{M}_{1}:\left\{\eta_{p}: p \geq 0\right\} \subset A(f, x) \Rightarrow \overline{\left\{\eta_{p}: p \geq 0\right\}}=A_{0}(f) \subset A(f, x)$.

The converse inclusion follows from proposition 2.5.10, where the hypothesis of recurrence is used. There exists comeager set $\bar{M}_{2}$ of transitive points. Therefore, $\forall x \in \bar{M}_{2}, A(f, x) \subset A_{0}(f)=$ $\cup_{z \text { transtive }} A(f, z)$.

Finally, considering the comeager set $\bar{M}_{\star}=\bar{M}_{1} \cap \bar{M}_{2}$, we have that $A_{0}(f) \subset A(f, x)$ and $A(f, x) \subset$ $A_{0}(f)$. In conclusion $A(f, x)=A_{0}(f), \forall x \in \bar{M}_{\star}$.

Lemma (Accumulation characterization). Let $\phi_{n}: \bar{M} \rightarrow Y, n \geq 0$, with $Y$ a metric space, be a sequence of functions. Given $y \in Y$, define $X\left(\left(\phi_{n}\right)_{n}, y\right)=\left\{x \in \bar{M}:\left(\phi_{n}(x)\right)_{n \geq 1}\right.$ accumulates in $\left.y\right\}$.

Then $X\left(\left(\phi_{n}\right)_{n}, y\right)=\bigcap_{N, k \geq 1} \bigcup_{n \geq N} \phi_{n}^{-1}(B(y, 1 / k+1))$.

Proof. Choosing $x \in X\left(\left(\phi_{n}\right)_{n}, y\right) \Rightarrow\left(\phi_{n}(x)\right)_{n \geq 1}$ accumulates in $y \Rightarrow \exists\left(n_{q}\right)_{q \geq 1}, n_{q} \nearrow \infty$, such that $\phi_{n_{q}}(x) \rightarrow y \Rightarrow \forall N, k \in \mathbb{N}, \exists Q_{1}$ such that, $\forall q \geq Q_{1} \geq 1$, we have $n_{q} \geq N$ and $\exists Q_{2} \geq 1$ such that, $\forall q \geq Q_{2}$, we have $\phi_{n_{q}}(x) \in B(y, 1 / k+1)$, i.e, $x \in \phi_{n_{q}}{ }^{-1}(B(y, 1 / k+1))$. Choosing $q \geq Q=\max \left\{Q_{1}, Q_{2}\right\}$, and denoting $n_{q}=n(N, k)$, we have that $n(N, k) \geq N$ and $x \in \phi_{n(N, k)}{ }^{-1}(B(y, 1 / k+1)) \Rightarrow x \in$ $\cup_{n \geq N} \phi_{n}{ }^{-1}(B(y, 1 / k+1))$. Since $n$ and $k$ were chosen arbitrarily, $x \in \cap_{N, k} \cup_{n \geq N} \phi_{n}^{-1}(B(y, 1 / k+1))$, as desired.

Reciprocally, $x \in \cap_{N, k} \cup_{n \geq N} \phi_{n}{ }^{-1}(B(y, 1 / k+1)) \Rightarrow \forall N, k, x \in \cup_{n \geq N} \phi_{n}{ }^{-1}(B(y, 1 / k+1)) \Rightarrow \forall N, k$, $\exists n(N, k) \geq N$ such that $\phi_{n(N, k)}(x) \in B(y, 1 / k+1)$ and, by letting $n_{1}=n(1,1), n_{q}=n\left(n_{q-1}+\right.$ 
$1, q), \forall q \geq 1$, we obtain $\left(n_{q}\right)_{q \geq 1}, n_{q} \nearrow \infty$ (since $\left.n_{q} \geq n_{q-1}+1\right)$, such that $\phi_{n_{q}}(x) \rightarrow y$ (since $\left.\phi_{n_{q}}(x) \in B(y, 1 / q+1), q \geq 1\right)$. Therefore $x \in X\left(\left(\phi_{n}\right)_{n}, y\right)$, as desired.

According to propositions 2.5.8 and 2.5.9, given the hypothesis of the theorem, if $X$ is infinite or if $X$ has no isolated points (the first case implying the second), every point $z$ indexing the union $A_{0}(f)=\cup_{z \text { transitive }} A(f, z)$ is of the same type of $z_{0}$, i.e., not only transitive but also recurrent.

Since $A_{0}(f)$ is non-empty and comprised of probabilities accumulated by the $\alpha_{f}(\cdot)_{n}$ 's, as already discussed while arguing for theorem 2.4.3, it should be that all measures in $A_{0}(f)$ are invariant, thus $A_{0}(f) \subset \mathcal{M}_{1}(f)$.

Whenever $A_{0}(f)=\mathcal{M}_{1}(f)$, we say the dynamical system $f$ is of maximal oscillation. Also, whenever a point $x \in X$ is such that $A(f, x)=\mathcal{M}_{1}(f)$, we say $x$ is a point of maximal oscillation - i.e., of "worst" divergence for the Birkhoff average ${ }^{1}$. If the dynamical system admits only one invariant measure, that would consist a trivial case of maximal oscillation (as discussed, $A_{0}(f)$ is always non-empty). In this case, the notion of "worst divergence" is not really appropriate.

Many other examples of maximal oscillation are well-known. An important one is the unilateral full shift on the product space $X^{\mathbb{N}}, X$ finite, equipped with the product topology and $\sigma$-algebra. A proof of such fact is presented in a classic text by Denker, Grillenberger and Sigmund [DGS76], using the so called "specification property" (see [DGS76], proposition 21.2) and that this property implies maximal oscillation (see [DGS76], proposition 21.18). Since this example is pretty much conventional, and its demonstration involves throwing in additional technicalities, we prefer to omit its proof.

Examples of non maximal oscillation dynamical systems exist and are more delicate, so one will be presented later.

The paper by Winkler in which we are basing this discussion [Win10] indicates that theorem 3.2.1 admits some generalization, using no transitivity and giving conclusions another, more general, clothing. One such result would be something in the direction of an analogue to theorem 3.1.4. However, such a generalization requires a much broader framework and the associated analogy to be obtained is less striking. For these reasons this discussion is omitted. A more recent, still unpublished, work of the same author along these lines addresses this issue [Win]. This paper also cites the classic text by Denker, Grillenberger and Sigmund [DGS76] for the construction of this generalization.

However, as theorem 3.2.1 is stated, transitivity is necessary. If it is dropped, the following counterxample is available.

Example 3.2.1 (Transitivity is necessary). Let $X=[0,1]$ with the standard topology and $f(x)=x$. There are no transitive points, so $A_{0}(f)=\varnothing$. But every point $x$ is such that $A(f, x)=\left\{\delta_{x}\right\}$.

As stated, recurrence also is necessary. If it is dropped, a counterxample is available.

Before presenting the counterexample, we should answer the question: The set $\bar{M}_{\star}$ presented in the proof is comprised of transitive points. Is every transitive point in this set?

The answer is no. Consider the full shift $\sigma$ on $X^{\mathbb{N}}=\{0,1\}^{\mathbb{N}}$. As we said, it is of maximal oscillation. Now consider a sequence $x_{0}$ containing every finite word, each separated by sufficiently long blocks of 0's. As such, $x_{0}$ is transitive and recurrent, but $A\left(\sigma, x_{0}\right)=\left\{\delta_{0^{\infty}}\right\}$, where $0^{\infty}=(0,0, \ldots)$. So, clearly, $x_{0} \notin \bar{M}_{\star}$, once $A\left(\sigma, x_{0}\right)=\left\{\delta_{0^{\infty}}\right\} \neq \mathcal{M}_{1}(\sigma)=A_{0}(\sigma)$.

Example 3.2.2 (Recurrence is necessary). Consider $X=X_{0} \cup X_{1}$, where $X_{0}=\{0,1\}^{\mathbb{N}}$ with the product topology and $\sigma$-algebra (see discussion around theorem 2.1.41) is adjoined with $X_{1}=\left\{x_{1}\right\}$, consisting of an isolated point. Consider the dynamical system $\tilde{\sigma}: X \rightarrow X$ given by $\left.\tilde{\sigma}\right|_{X_{0}}=\sigma$, the unilateral full shift on $\{0,1\}^{\mathbb{N}}$, and $\tilde{\sigma}\left(x_{1}\right)=x_{0}$, where $x_{0}$ is the point in $\{0,1\}^{\mathbb{N}}$ described before this example.

As such, $x_{1}$ is transitive but not recurrent and $A\left(f, x_{1}\right)=A\left(f, x_{0}\right)=\left\{\delta_{0^{\infty}}\right\}$. Since $\left\{x_{1}\right\}$ is an open set, $x_{1}$ is the only transitive point, thus $A_{0}(\tilde{\sigma})=\left\{\delta_{0^{\infty}}\right\}$.

${ }^{1}$ The Birkhoff average being considered here is not $1 / n \sum_{j=0}^{n-1} \varphi \circ f^{j}(x)$, but $1 / n \sum_{j=0}^{n-1} \delta_{f^{j}(x)}$ 
However, as we know, for $y$ 's in a comeager set $Y_{0} \subset X_{0} \subset X, A(\tilde{\sigma}, y)=\overline{A_{0}(\sigma)} \neq\left\{\delta_{0^{\infty}}\right\}=A_{0}(\tilde{\sigma})$, where $\overline{A_{0}(\sigma)}$ is comprised of the same measures on $A_{0}(\sigma)$ but extended to assign measure 0 to $\left\{x_{1}\right\}$. Since $Y_{0} \subset X$ is non meager, the characterization that $A(\tilde{\sigma}, x)=A_{0}(\tilde{\sigma})$ holds in a comeager set is invalid.

We close this section presenting an example of non maximal oscillation.

Example 3.2.3 (Non maximal oscillation). Consider the sequence $x=\left(a_{n}\right)_{n \geq 0}$ in $\{0,1\}^{\mathbb{N}}$, which we may write as a (binary) "word" $a_{0} a_{1} a_{2} a_{3} \ldots$, whose $a_{n}$ 's are defined to satisfy the remarkable property that $a_{0} a_{1} a_{2} a_{3} \ldots=0^{1} 1^{1} a_{0} 0^{2} 1^{1} a_{0} a_{1} 0^{3} 1^{2} a_{0} a_{1} a_{2} \ldots$, where $0^{k}$ means a finite word of $k$ consecutive 0 's, likewise for $1^{k}$. This definition implies $a_{0}=0, a_{1}=1, a_{2}=a_{0}=0, a_{3}=0, a_{4}=0, a_{5}=1, a_{6}=1, a_{7}=$ $a_{0}=0, a_{8}=a_{1}=1$, etc - see below:

$\begin{array}{llllllllllllllllll}a_{0} & a_{1} & a_{2} & a_{3} & a_{4} & a_{5} & a_{6} & a_{7} & a_{8} & a_{9} & a_{10} & a_{11} & a_{12} & a_{13} & a_{14} & a_{15} & a_{16} & a_{17} \\ 0 & 1 & a_{0} & 0 & 0 & 1 & 1 & a_{0} & a_{1} & 0 & 0 & 0 & 1 & 1 & 1 & a_{0} & a_{1} & a_{2} \\ 0 & 1 & \underline{0} & 0 & 0 & 1 & 1 & \underline{0} & \underline{1} & 0 & 0 & 0 & 1 & 1 & 1 & \underline{0} & \underline{1} & \underline{0}\end{array}$

Consider $x$ in the context of the unilateral shift $\sigma$ on $\{0,1\}^{\mathbb{N}}$ equipped with the product topology and $\sigma$-algebra. As discussed around theorem 2.1.41, the product topology is compact, metrizable and admits as basis the family of elementary cylinders $\left[m ; b_{m}, \ldots, b_{m+k}\right]=\left\{x=\left(x_{0}, x_{1}, \ldots\right) \in\{0,1\}^{\mathbb{N}}\right.$ : $\left.x_{m}=b_{m}, \ldots, x_{m+k}=b_{m+k}\right\}, m \geq 0, k \geq 0, b_{j} \in\{0,1\}$ for $m \leq j \leq m+k$.

An important consequence of the definition of $x$ is that it is recurrent. For instance, for every $k \geq 0$, the first $k$ entries of $x, a_{0} \ldots a_{k-1}$, coincide with the first $k$ entries of a large enough iterate of $x$.

Now let $X \subset\{0,1\}^{\mathbb{N}}$ be the subset of points accumulated by the (sequence) orbit of $x$. Clearly $1^{\infty} \in X$, where $1^{\infty}=111 \ldots$ Also, $x$ is transitive in $X$.

Now we consider the dynamical system $f=\left.\sigma\right|_{X}: X \rightarrow X$ (it is well defined, since the shifts of $x$ or of any such accumulation points are still accumulated by the orbit of $x$ ), where $X$ is endowed with the restricted topology and $\sigma$-algebra. This dynamical system is called a subshift generated by $x$.

Clearly, $f$ is continuous, because if two points are close together, their initial digits will be identical, what is still verifiable after the shift.

Again clearly, $X$ is a metric space, as a subset of $\{0,1\}^{\mathbb{N}}$. It is also a compact space, as a (closed) set of accumulation points of an orbit within the compact metric space $\{0,1\}^{\mathbb{N}}$.

Finally, once $x \in X$ is transitive and recurrent, we are in the realm of theorem 3.2.1.

Note $1^{\infty} \in X$ is a fixed point for $f$, thus $\delta_{1^{\infty}} \in \mathcal{M}_{1}(f)$. We want to show that $\delta_{1^{\infty}} \notin A_{0}(f)$. In this case, $A_{0}(f) \neq \mathcal{M}_{1}(f)$, so $f$ would not be of maximal oscillation, as desired.

The sequence $\left(a_{n}\right)_{n \geq 0}$ provides a natural partition of $\mathbb{N}$ into subintervals $I_{k}=I_{k}^{(0)} \dot{\cup} I_{k}^{(1)} \dot{\cup} I_{k}^{(r)}$, $k \geq 1$. They are such that:

(a) elementwise, $I_{1}<I_{2}<\ldots$ and $I_{k}^{(0)}<I_{k}^{(1)}<I_{k}^{(r)} ; k \geq 1$,

(b) $\# I_{k}^{(0)}=\# I_{k}^{(1)}=\# I_{k}^{(r)}=k, k \geq 1$, thus $\# I_{k}=3 k$,

(c1) $n \in I_{k}^{(0)} \Rightarrow a_{n}=0$,

(c2) $n \in I_{k}^{(1)} \Rightarrow a_{n}=1$,

(c3) writing $I_{k}^{(r)}=\{m, \ldots, m+k-1\}$ and $n \in I_{k}^{(r)}$ as $n=m+j$, for some $j \in[0, k-1] \cap \mathbb{N}$, we have that $n \in I_{k}^{(r)} \Rightarrow a_{n}=a_{j}$.

The following table describes what is going on.

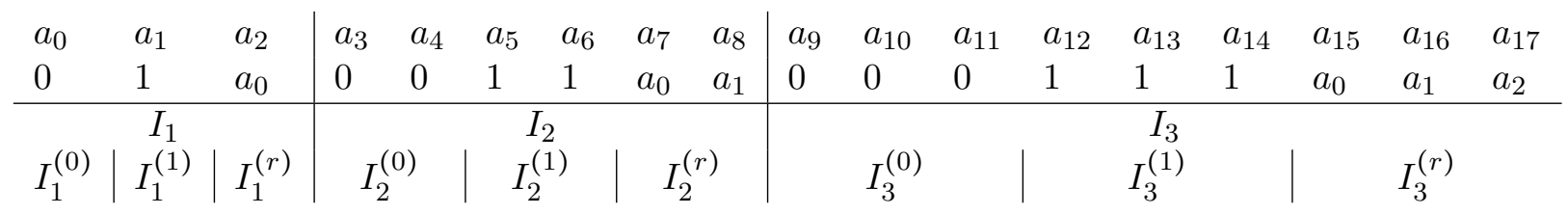


Now let $W_{l}$ be the set of finite subwords of $x_{0}$ with length $l$, such as $w=\left(a_{m}, a_{m+1}, \ldots, a_{m+l-1}\right)$. Let $W=\cup_{l \geq 0} W_{l}$. For a finite word $w=\left(a_{0}^{\prime}, a_{1}^{\prime}, \ldots, a_{l-1}^{\prime}\right)$, we let $d(1 \mid w)=\frac{\#\left\{j \in[0, l-1] \cap \mathbb{N}: a_{j}^{\prime}=1\right\}}{l}$ denote the frequency of 1 's in $w$ and its complement $d(0 \mid w)=1-d(1 \mid w)$ denote the frequency of 0 's in $w$.

Lemma (First lemma). Every initial word of $x, w=\left(a_{0}, \ldots, a_{l-1}, l \geq 1\right.$, satisfies $d(1 \mid w) \leq 1 / 2 \leq$ $d(0 \mid w)$.

Proof. It is clear that the statement holds in every finite initial word with indexes up until $I_{1}$.

Now every finite initial word with indexes up until $I_{2}$ is (at most) a concatenation of $\left(a_{0} \ldots a_{3 \cdot 1}\right)$ with blocks of 0 and 1 (of the same length and in this order) and, again a word $\left(a_{0}, \ldots, a_{l}\right)$, with $l \in[0,3 \cdot 1] \cap \mathbb{N}$. By the last argument, the words concatenated in the beginning and the end satisfy the conclusion, so the same can be said for the larger word under consideration.

Repeating the argument:

Every finite initial word with indexes up until $I_{k}$ is (at most) a concatenation of $\left(a_{0} \ldots a_{3 \cdot(k-1)}\right)$ with blocks of 0 and 1 (of the same length and in this order) and, again a word $\left(a_{0}, \ldots, a_{l}\right)$, with $l \in[0,3 \cdot k-1] \cap \mathbb{N}$. By the last argument, the words concatenated in the beginning and the end satisfy the conclusion, so the same can be said for the larger word under consideration.

Lemma (Second lemma). Every $w \in W$ starting with 10, i.e., $w=10 w^{\prime}$, satisfies $d\left(1 \mid 0 w^{\prime}\right) \leq 1 / 2 \leq$ $d\left(0 \mid 0 w^{\prime}\right)$.

Proof. Suppose not. Then there exists "counterexample" words $w=\left(a_{m}, a_{m+1}, \ldots, a_{m+l-1}\right) \in W$, $w=10 w^{\prime}$, such that $d\left(1 \mid 0 w^{\prime}\right)>1 / 2>d\left(0 \mid 0 w^{\prime}\right)$. Each of these starts at a natural number $m \geq 0$. Let $m_{0}$ be the smallest $m$ among those words. In the group of those starting with $m=m_{0}$, each has a natural length $l \geq 2$. Let $l_{0}$ be the smallest $l$ among the words in this last group. So we have a "minimal" word $w=\left(a_{m_{0}}, a_{m_{0}+1}, \ldots, a_{m_{0}+l_{0}-1}\right) \in W, w=10 w^{\prime}$, such that $d\left(1 \mid 0 w^{\prime}\right)>1 / 2>d\left(0 \mid 0 w^{\prime}\right)$.

Clearly, $m_{0} \notin I_{k}^{(0)}$, because it starts with 1 . We are left with the following two cases:

1) $m_{0} \in I_{k_{0}}^{(1)}$, for some $k_{0} \geq 1$ : In this case, $m_{0}$ has to be in the rightmost end of $I_{k_{0}}^{(1)}$, otherwise the first 1 of $w$ would not be followed by a 0 . Except for the first entry, $w$ has $l_{0}-1$ entries left to the right. The first subcase is $l_{0}-1 \leq k_{0}$. Here, $m_{0}+l_{0}-1 \in I_{k_{0}}^{(r)}, w=1 a_{0} a_{1} \ldots a_{l_{0}-1}$, so $0 w^{\prime}=a_{0} a_{1} \ldots a_{l_{0}-1}$ and, by the first lemma, $d\left(0 \mid 0 w^{\prime}\right) \geq 1 / 2$ - a contradiction. In the second subcase, $l_{0}-1>k_{0}$. Here, $0 w^{\prime}$ is (at most) a concatenation of $\left(a_{0} \ldots a_{k_{0}}\right)$ with successive strings comprised of: blocks of 0 and 1 (of the same length and in this order) followed by initial words. By the first lemma, $\left(a_{0} \ldots a_{k_{0}}\right)$ and the successively appended strings have a frequency of 0 's greater than or equal to $1 / 2$, so $d\left(0 \mid 0 w^{\prime}\right) \geq 1 / 2$ - a contradiction.

2) $m_{0} \in I_{k_{0}}^{(r)}$, for some $k_{0} \geq 1$ : In the first subcase, $m_{0}+l-1 \in I_{k_{0}}^{(r)}$ and the word $w$ is entirely in the block indexed by $\in I_{k_{0}}^{(r)}$, case in which we could always extend is to left left and consider $\bar{w}=\left(a_{m_{1}}, a_{m_{1}+1}, \ldots, a_{m_{0}+l-1}\right)=10 \bar{w}^{\prime}$ (see, the block of 1 's in $I_{k}^{(1)}$ is always followed by $0=a_{0}$ ), where $m_{1}<m_{0}$ is the largest index of $I_{k_{0}}^{(1)}$, and $\bar{w}$ would qualify as a "counterexample" word (as in the first subcase of case 1) - contradicting the minimality of $m_{0}$. In the second subcase, the remaining one, $w$ is the concatenation of $a_{m_{0}} \ldots a_{m_{0}+q}$ (where $m_{0}+q$ is the largest index of $I_{k_{0}}^{(r)}$ ) with sucessive strings comprised of: blocks of 0 and 1 (of the same length and in this order) followed by initial words. Since the word $a_{m_{0}} \ldots a_{m_{0}+q}=10 w^{\prime \prime}$ starts at $m_{0}$ and has length smaller then $l_{0}$ it can not be a "counterexample", i.e, $d\left(0 \mid 0 w^{\prime \prime}\right) \geq 1 / 2$. By the first lemma, the successively appended strings also have frequency of 0 's greater than or equal to $1 / 2$, so $d\left(0 \mid 0 w^{\prime}\right) \geq 1 / 2-$ combining these two findings, we have a contradiction.

This last lemma is the one to solve our problem.

Lemma (Third lemma). Every $y=\left(b_{n}\right)_{n \geq 0} \in X$ either:

type 1) is of the form $y=u 1^{\infty}$, where $u$ is a finite word

type 2) has upper density of 1 's, $\bar{d}(1 \mid y)$, at most $1 / 2$, where $\bar{d}(1 \mid y)=\lim \sup _{n} d\left(1|y|_{n}\right)$ with $\left.y\right|_{n}=$ $b_{0} \ldots b_{n-1}$.

In particular, $\delta_{1^{\infty}} \notin A(f, y)$ for every $y$ of type 2 . 
Proof. Suppose $y$ is not of type 1 . Then $y$ should have infinitely many 0 's. If $y=0^{\infty}$, it is clearly of type 2. Otherwise, it should have somewhere a 1 followed by a 0 , so $y=u 10 y^{\prime}$, where $u$ is a finite word.

If we take $y^{\prime \prime}$ to be any finite initial word of $y^{\prime}, 10 y^{\prime \prime} \in W$, because $u 10 y^{\prime} \in X \Rightarrow 10 y^{\prime} \in X$ (after taking a finite iterations of the shift $f$ ), and so $x$ accumulates in $10 y^{\prime}$, in particular, its finite initial word $10 y^{\prime \prime}$ should be in the string of $x$, hence in $W$. As such, and starting with 10 , by the second lemma, $d\left(1 \mid 0 y^{\prime \prime}\right) \leq 1 / 2$.

Since the length of $10 y^{\prime \prime}$ was arbitrary, the last conclusion drives the long term behavior of the word $y$ to satisfy $\bar{d}(1 \mid y) \leq 1 / 2$. In this case, $y$ is of type 2 , as desired.

Now take a point $y$ of type 2 and $\nu \in A(f, y)$. In this case, $\alpha_{f}(y)_{n_{k}} \stackrel{w *}{\longrightarrow} \nu$, for some subsequence $\left(n_{k}\right)_{k \geq 0}$. Due to proposition 2.2.1 and denoting the open set $\left\{z=\left(c_{n}\right)_{n \geq 0} \in X: c_{0}=1\right\}$ by $Z_{1}$, we have $\limsup _{k} d\left(1|y|_{n_{k}}\right) \quad \geq \liminf _{k} d\left(1|y|_{n_{k}}\right) \quad=\quad \liminf _{k} \frac{\#\left\{j \in\left[0, n_{k}-1\right] \cap \mathbb{N}: f^{j}(y) \in Z_{1}\right\}}{n_{k}}=$ $\liminf _{k}\left(1 / n_{k} \sum_{j=0}^{n_{k}-1} \delta_{f^{j}(y)}\right)\left(Z_{1}\right) \geq \nu\left(Z_{1}\right)$ (for this last inequality, see 2.2.1-(iii)). Since $y$ is of type 2, $1 / 2 \geq \lim \sup _{k} d\left(1|y|_{n_{k}}\right)$, so $\nu\left(Z_{1}\right) \leq 1 / 2$.

Once $1^{\infty} \in Z_{1}, \delta_{1^{\infty}}\left(Z_{1}\right)=1$, so $\delta_{1^{\infty}} \notin A(f, y)$, for every $y$ of type 2 .

To conclude the example, we note that elements $y$ of type 1 in $X$ are countable. Hence elements $y$ of type 2 in $X$ comprise a comeager set. Since $\delta_{1^{\infty}} \notin A(f, y)$ for $y$ 's in a comeager set, there is no way for $\delta_{1^{\infty}}$ to be in $A_{0}(f)$, once we take into account theorem 3.2.1. Therefore $\delta_{1^{\infty}} \notin A_{0}(f)$, as desired.

Completed the example, before closing the section, some concluding remarks are worthwhile.

Compressing this section in few words, we could say that a topological (topological dynamics) analogue for Birkhoff's ergodic theorem (assuming ergodicity) (see theorem 3.1.8) was obtained. It assumed transitivity and recurrence rather than ergodicity. Instead of convergence of Birkhoff averages $^{2}$ being typical, it states the opposite: a dramatic non-convergence of Birkhoff averages is the (topological) typical behavior, as they will accumulate in a rather large set, $A_{0}(f)$ (see theorem 3.2.1).

This characterization is (invariant) measure-free and the extent of the former set is a topological property of the dynamical system. Whenever the last set coincides with that of invariant measures, we say the system is of maximal oscillation. Whenever the Birkhoff averages starting from a point accumulate in the set of invariant measures, this point is called a maximal oscillation point. These points are of "worst" divergence in the sense of Birkhoff averages and are typical in maximal oscillation systems.

Let the regular set be the subset of the ambient space $X$ whose Birkhoff averages converge (as measures with respect to the weak* topology), and its complement be the irregular set. Under the joint hypothesis of Birkhoff's ergodic theorem (with ergodicity) and its analogue (see theorems 3.1.8 and 3.2.1), and assuming $A_{0}(f)$ is not a singleton, we can conclude that the regular set is measure-theoretically large (by theorem 3.1.8) but topologically small (by theorem 3.2.1), while the irregular set is measure-theoretically small but topologically large. This is a quite interesting contrast, in line with those comparisons between measure and category explored in [Oxt96]. In this context, one can use the characterization provided by the analogue just presented to say something about what happens to Birkhoff averages out of the domain of Birkhoff's ergodic theorem.

Many works explore topological connections with Birkhoff's ergodic theorem from this perspective, analyzing when the irregular set ought to be topologically large [LW14, BLV14], or when the set of points of maximal oscillation ought to be topologically large [DGS76, LW16]. In the same flavor, an extensive literature analyzed, under certain circumstances, how large can be irregular sets from the other points of view, such as topological entropy, topological pressure [EKL05, Tho10] and Hausdorff dimension [BS00].

This piece of literature aims to describe how large are irregular sets from many points of view, but not to characterize this irregularity, i.e., how Birkhoff averages starting from points in this set

\footnotetext{
${ }^{2}$ The Birkhoff average being considered here is not $1 / n \sum_{j=0}^{n-1} \varphi \circ f^{j}(x)$, but $1 / n \sum_{j=0}^{n-1} \delta_{f^{j}(x)}$
} 
accumulate. However, the topological analogue discussed here provides such characterization (in a topologically large set).

As listed, some of those points of view are new to the sort of analysis herein developed, e.g., Hausdorff dimension. Prospectively, it can be considered whether some sort of characterization of Birkhoff averages outside the domain of Birkhoff's ergodic theorem and its analogue can be obtained, e.g, in a subset which is kind of large from the Hausdorff dimension viewpoint. 


\section{Chapter 4}

\section{Law of Large Numbers: From Probability to Ergodic Theory}

This chapter leverages in many of the ideas discussed in chapter 2 and section 3.1. However, ideas in this chapter are developed by the author with no reference to any text. The only exception is the proof of the laws of large numbers adapting the probabilistic machinery from [Çin11].

\subsection{Unifying Notation and Framework}

The law of large numbers, to be proved in this section, is a result in probability theory. Since our intent is to apply it to ergodic theory, we need to fit the ergodic theory framework within that of probability theory and vice versa.

This joint framework is described as follows.

Let (i) $(X, \mathcal{A})$ be a measurable space (Borelian if $X$ has a topology), (ii) $\psi:(X, \mathcal{A}) \rightarrow\left(\mathbb{R}, \mathcal{B}_{\mathbb{R}}\right)$ be a measurable function (understood both as an observable or as a random variable) and (iii) $f:(X, \mathcal{A}) \rightarrow(X, \mathcal{A})$ be a measurable dynamical system equipped with (iv) a probability measure $\mu$ on $(X, \mathcal{A})$ such that $(f, \mu)$ is an invariant system.

From these primitives, an underlying probability space $(X, \mathcal{A}, \mu)$ is always available. However, the probability $\mu$ does not need to be understood as fixed, as we may be interested varying $\mu$ in $\mathcal{M}(f)$ (or other sets). Nonetheless, one or a family of those probabilities should always be considered during the study and context should make it clear.

Clearly, $\psi \circ f^{j}$ is measurable for all $j \geq 0$, so, as in the probability section, a joint measurable function can be derived: $\bigotimes_{j=0}^{\infty} \psi \circ f^{j}:(X, \mathcal{A}) \rightarrow\left(\mathbb{R}^{\infty}, \bigotimes_{j=0}^{\infty} \mathcal{B}_{\mathbb{R}}=\mathcal{B}_{\mathbb{R}^{\infty}}\right)$ given by $x \mapsto\left(\psi \circ f^{j}(x)\right)_{j \geq 0}=$ $\left(\psi(x), \psi(f(x)), \ldots, \psi\left(f^{j}(x)\right), \ldots\right)$, i.e., mapping $x$ to its $\psi$-observed $f$-orbit.

The following diagram summarizes all of the above:

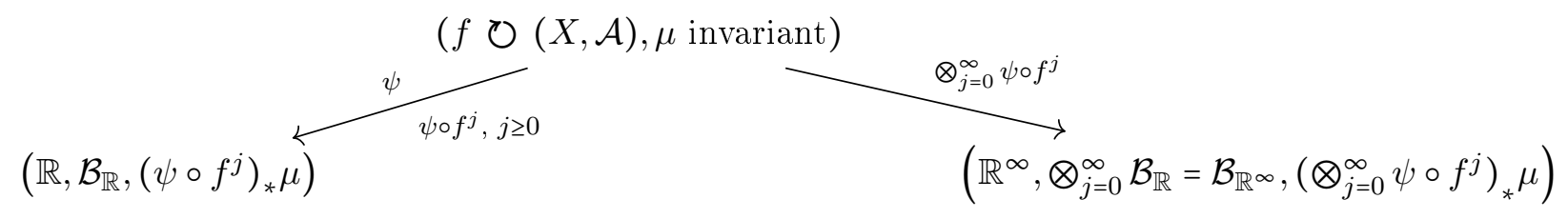

We already defined independence of a family of measurable functions in section 2.3 (see definition 2.3.5 and proposition 2.3.7). In the special case where we consider the family of measurable functions to be $\psi_{n}=\psi \circ f^{n}, n \geq 0$, the characterization of proposition 2.3.7 resumes to:

$\mu\left(\psi^{-1}\left(B_{0}\right) \cap \ldots \cap\left(\psi \circ f^{k-1}\right)^{-1}\left(B_{k-1}\right)\right)=\mu\left(\psi^{-1}\left(B_{0}\right)\right) \cdot \ldots \cdot \mu\left(\left(\psi \circ f^{k-1}\right)^{-1}\left(B_{k-1}\right)\right) \Leftrightarrow \mu\left(\psi^{-1}\left(B_{0}\right) \cap \ldots \cap\right.$ $\left.f^{-(k-1)}\left(\psi^{-1}\left(B_{k-1}\right)\right)\right)=\mu\left(\psi^{-1}\left(B_{0}\right)\right) \cdot \ldots \cdot \mu\left(f^{-(k-1)}\left(\psi^{-1}\left(B_{k-1}\right)\right)\right)$, verified $\forall\left(B_{0}, \ldots, B_{k-1}\right)$, such that $B_{j} \in \mathcal{B}_{\mathbb{R}}, 0 \leq j \leq k-1$ (or $B_{j} \in \mathcal{G}_{j}, 0 \leq j \leq k-1$, if each $\mathcal{G}_{j}$ generates $\mathcal{B}_{j}$ ). 
Definition 4.1.1 (Ergodicity with respect to a system and of a system). Let $(X, \mathcal{A})$ be a measurable space, $f:(X, \mathcal{A}) \rightarrow(X, \mathcal{A})$ be a measurable dynamical system and a $\mu$ be probability measure on $(X, \mathcal{A})$ such that $(f, \mu)$ is an invariant system.

Inspired by proposition 3.1.6-(iii), we say a measurable function in $L^{1}(\mu, \mathbb{R}), \psi: X \rightarrow \mathbb{R}$, is ergodic with respect to $(f, \mu)$, or, in short, that $(\psi,(f, \mu))$ is ergodic, if $1 / n \sum_{j=0}^{n-1} \psi \circ f^{j}(x) \rightarrow \int \psi d \mu$, 4-a.e. $x \in X$.

As we know from proposition 3.1.6, we say $(f, \mu)$ is an ergodic system if $(\psi,(f, \mu))$ is ergodic for every $\psi \in L^{1}(\mu, \mathbb{R})$.

Above, we rephrased the definition of ergodicity in order to motivate and add perspective to the following definition.

Definition 4.1.2 (Independence and identical distribution with respect to a system and of a system). Let $(X, \mathcal{A})$ be a measurable space, $f:(X, \mathcal{A}) \rightarrow(X, \mathcal{A})$ be a measurable dynamical system and $a \mu$ be probability measure on $(X, \mathcal{A})$ such that $(f, \mu)$ is an invariant system.

We say a measurable function, $\psi: X \rightarrow \mathbb{R}$, is independent and identically distributed (IID) with respect to $(f, \mu)$, or, in short, that $(\psi,(f, \mu))$ is IID, if $\left(\psi \circ f^{n}\right)_{n \geq 0}$ is IID.

We say $(f, \mu)$ is IID if $(\psi,(f, \mu))$ is ergodic for some non $\mu$-a.e. constant measurable $\psi: X \rightarrow \mathbb{R}$.

Remark. Note, in this last definition, we changed (a) $\psi \in L^{1}(\mu, \mathbb{R})$ to $\psi$ measurable (b) "every" to "some". Also note that demanding identical distribution in the previous definition is, in this context, a non-binding restriction. In fact, since $(f, \mu)$ is an invariant system, $\left(\left(\psi \circ f^{j}\right)_{*} \mu\right)=\psi_{*}\left(f_{*}^{j} \mu\right)=\psi_{\star} \mu$. Therefore only the "independence" part has impact here.

With this definition, we can show that an IID system $(f, \mu)$ is (measure theoretically) semiconjugated to a Bernoulli system, or, in other others, that Bernoulli is a (measure theoretical) factor of $(f, \mu)$. We will explain all of the concepts involved after the statement of the proposition.

Proposition 4.1.3 (IID systems have a Bernoulli factor). Let $(X, \mathcal{A})$ be a measurable space, $f$ : $(X, \mathcal{A}) \rightarrow(X, \mathcal{A})$ be a measurable dynamical system and a $\mu$ be probability measure on $(X, \mathcal{A})$ such that $(f, \mu)$ is an invariant system.

If $(f, \mu)$ is IID, then $(f, \mu)$ has Bernoulli as a (measure theoretical) factor.

The Bernoulli shift we will consider is exactly the one discussed in example 3.1.9, where the base space is $\{0,1\}$, equipped with the discrete $\sigma$-algebra and the measure $\beta_{p}$, such that $\beta_{p}(\{1\})=p$ (a special case of the example discussed after theorem 2.1.41, using a finite base space). This system is invariant and ergodic. We will specify an appropriate $p$ when it is time.

Let $(X, \mathcal{A})$ and $(Y, \mathcal{B})$ be measure spaces, $f: X \rightarrow X$ and $g: Y \rightarrow Y$ be measurable dynamical system and $\mu$ and $\nu$ be measures on $(X, \mathcal{A})$ and $(Y, \mathcal{B})$, respectively, such that $(f, \mu)$ and $(g, \nu)$ are invariant systems. What we mean when we say $(g, \nu)$ is (measure theoretical) factor of $(f, \mu)$ is that there exists a function $\pi: X \rightarrow Y$, called a semi-conjugacy, or projection, which (a) is measurable and measure preserving transformation (whose image has outer measure 1) and (b) verifies $\pi \circ f=g \circ \pi$, making the following diagram commute:

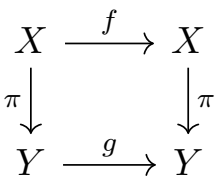

When plugging in our original systems $(f, \mu)$ and $\left(\sigma, \beta_{p}^{\mathbb{N}}\right)$, to obtain the desired $\pi$ we proceed presented in the following proof.

Proof. Let $\psi: X \rightarrow \mathbb{R}$ be the non $\mu$-a.e. constant measurable function obtained from the IID definition.

We claim that $\exists r_{0} \in \mathbb{R}$ such that $0<\mu\left(\psi^{-1}\left(\left(-\infty, r_{0}\right]\right)\right)<1$. Suppose not. If there was no such $r_{0}$ : $\forall r \in \mathbb{R}, \mu\left(\psi^{-1}((-\infty, r])\right) \in\{0,1\}$. However, since $\lim _{r \rightarrow-\infty} \mu\left(\psi^{-1}((-\infty, r])\right)=0$, $\lim _{r \rightarrow \infty} \mu\left(\psi^{-1}((-\infty, r])\right)=1, \psi_{*} \mu$ is $\{0,1\}$-valued and the sets $(-\infty, p]$ are nested (as $p$ increases), there should exist a $r_{1} \in \mathbb{R}$ such that $\mu\left(\psi^{-1}((-\infty, r])\right)=0, \forall r<r_{1}$ and $\mu\left(\psi^{-1}((-\infty, r])\right)=1, \forall r>r_{1}$. 
Then a standard argument implies that $\mu\left(\psi^{-1}\left(\left\{r_{1}\right\}\right)\right)=1-$ a contradiction, since $\psi$ is not $\mu$-a.e. constant.

Denote $\mu\left(\psi^{-1}\left(\left(-\infty, r_{0}\right]\right)\right)$ by $\alpha, \psi^{-1}\left(\left(-\infty, r_{0}\right]\right)$ by $X_{1}$, its complement by $X_{0},\left(-\infty, r_{0}\right]$ by $\tilde{X}_{1}$ and its complement by $\tilde{X}_{0}$. In $\beta_{p}^{\mathbb{N}}$, we will use $p$ as $\alpha$. Then we denote the characteristic function $\mathcal{X}_{X_{1}}: X \rightarrow\{0,1\}$ simply by $c$ and call it the coding function. We also use the orbit function $\theta: x \mapsto\left(x, f(x), f^{2}(x), \ldots\right)$. Finally, we let $\pi: X \rightarrow\{0,1\}^{\mathbb{N}}$ be given by $c \circ \theta$, where this composition should be understood as componentwise composition.

The property (b) of the projection function, that $\pi \circ f=\sigma \circ \pi$, is immediate here:

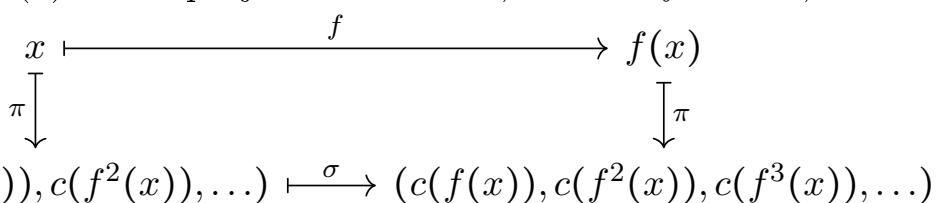

$$
\left(c(x), c(f(x)), c\left(f^{2}(x)\right), \ldots\right) \stackrel{\sigma}{\longmapsto}\left(c(f(x)), c\left(f^{2}(x)\right), c\left(f^{3}(x)\right), \ldots\right)
$$

It is left to prove those properties in (a).

To prove $\pi$ is measurable, it suffices to check that preimages of thin cylinders are measurable. Let $\left[m ; b_{m}, \ldots, b_{m+k}\right], b_{j} \in\{0,1\}$, be a thin cylinder. Then $\pi^{-1}\left(\left[m ; b_{m}, \ldots, b_{m+k}\right]\right)=\left\{x \in X: f^{m}(x) \epsilon\right.$ $\left.X_{b_{m}}, \ldots, f^{m+k} \in X_{b_{m+k}}\right\}=f^{-m}\left(X_{b_{m}}\right) \cap \ldots \cap f^{-(m+k)}\left(X_{b_{m+k}}\right)$, which is measurable, once $X_{0}$ and $X_{1}$ are measurable sets and $f$ is measurable function.

To prove $\pi$ is measure preserving, it suffices to check it on thin cylinders. Note $\mu\left(\pi^{-1}\left(\left[m ; b_{m}, \ldots, b_{m+k}\right]\right)\right)=\mu\left(f^{-m}\left(X_{b_{m}}\right) \cap \ldots \cap f^{-(m+k)}\left(X_{b_{m+k}}\right)\right)=\mu\left(f^{-m}\left(\psi^{-1}\left(\tilde{X}_{b_{m}}\right)\right) \cap \ldots \cap\right.$ $\left.f^{-(m+k)}\left(\psi^{-1}\left(\tilde{X}_{b_{m+k}}\right)\right)\right) \stackrel{(*)}{=} \mu\left(f^{-m}\left(\psi^{-1}\left(\tilde{X}_{b_{m}}\right)\right)\right) \cdot \ldots \cdot \mu\left(f^{-(m+k)}\left(\psi^{-1}\left(\tilde{X}_{b_{m+k}}\right)\right)\right) \stackrel{(* *)}{=} \mu\left(\psi^{-1}\left(\tilde{X}_{b_{m}}\right)\right)$. $\ldots \cdot \mu\left(\psi^{-1}\left(\tilde{X}_{b_{m+k}}\right)\right)=\beta_{\alpha}\left(\left\{b_{m}\right\}\right) \cdot \ldots \cdot \beta_{\alpha}\left(\left\{b_{m+k}\right\}\right)=\beta_{\alpha}^{\mathbb{N}}\left(\left[m ; b_{m}, \ldots, b_{m+k}\right]\right)$, as desired. Equality $(*)$ is due to the definition of IID (see the characterization discussed before definition 4.1.1). Equality $(* *)$ is because $(f, \mu)$ is an invariant system.

One such semi-conjugacy allows for some ergodic properties (not discussed in this text) of the factor system to be inherited by the other, "larger", system. Not every property, though, is inherited. Next, we will see an example of an ergodic IID system and another example of a non-ergodic IID system (still having a Bernoulli system as cofactor), showing that ergodicity can be not inherited.

Example 4.1.4 (Bernoulli shifts as IID systems and a models for IID stochastic processes). Let $(X, \mathcal{A}, \mu)$ be probability space and $\left(\varphi_{n}: X \rightarrow \mathbb{R}\right)_{n \geq 0}$ an IID stochastic process, whose distributions are $\varphi_{n *} \mu=\nu$.

Consider $\theta=\bigotimes_{j=0}^{\infty} \varphi_{j}: X \rightarrow \mathbb{R}^{\mathbb{N}}$ mapping $x \mapsto\left(\varphi_{0}(x), \varphi_{1}(x), \ldots\right)$, the shift dynamical system on $\mathbb{R}^{\mathbb{N}}$ (see example 3.1.9) and the pushforward probability $\eta=\theta_{*} \mu$. It is not difficult to check that $\eta=\nu^{\mathbb{N}}$.

Due to Caratheodory's extension theorem (see 2.1.10), it suffices to check the equality over the generating algebra comprised of cylinders of the form $\left[m ; B_{m}, \ldots, B_{m+k}\right], m \geq 0, k \geq 0, B_{j} \in \mathcal{B}_{\mathbb{R}}$ for $m \leq j \leq m+k$. For instance, $\eta\left(\left[m ; B_{m}, \ldots, B_{m+k}\right]\right)=\mu\left(\theta^{-1}\left(\left[m ; B_{m}, \ldots, B_{m+k}\right]\right)\right)=\mu\left(\varphi_{m}^{-1}\left(B_{m}\right) \cap \ldots \cap\right.$ $\left.\varphi_{m+k}^{-1}\left(B_{m+k}\right)\right)=\mu\left(\varphi_{m}^{-1}\left(B_{m}\right)\right) \cdot \ldots \cdot \mu\left(\varphi_{m+k}^{-1}\left(B_{m+k}\right)\right)=\nu\left(B_{m}\right) \cdot \ldots \cdot \nu\left(B_{m+k}\right)=\nu^{\mathbb{N}}\left(\left[m ; B_{m}, \ldots, B_{m+k}\right]\right)$.

We already know the Bernoulli shift $\left(\sigma, \nu^{\mathbb{N}}\right)$ is invariant and ergodic (see example 3.1.9).

The Bernoulli shift $\left(\sigma, \nu^{\mathbb{N}}\right)$ provides a model for $\left(\varphi_{n}\right)_{n \geq 0}$ in the sense that, equipped with the projection observable $\psi: \mathbb{R}^{\mathbb{N}} \rightarrow \mathbb{R}$ given by $\left(x_{0}, x_{1}, \ldots\right) \mapsto x_{0}:$ (a) $\varphi_{n}=\psi \circ \sigma^{n} \circ \theta$ and (b) for any non-negative integers $i_{0}<\ldots<i_{q}$ the random variables $\left(\varphi_{i_{0}}, \ldots, \varphi_{i_{q}}\right)$ and $\left(\psi \circ \sigma^{i_{0}}, \ldots, \psi \circ \sigma^{i_{q}}\right)$ have the same distributions, namely, $\left(\varphi_{i_{0}}, \ldots, \varphi_{i_{q}}\right)_{\star} \mu$ and $\left(\psi \circ \sigma^{i_{0}}, \ldots, \psi \circ \sigma^{i_{q}}\right)_{\star} \eta$.

Property (a) is clear. For property (b), let $i_{0}<\ldots<i_{q}$ be arbitrary non-negative integers and $E_{i_{0}}, \ldots, E_{i_{q}}$ be arbitrary measurable sets on the real line. Note that $\eta\left(\left(\psi \circ \sigma^{i_{0}}, \ldots, \psi \circ \sigma^{i_{q}}\right)^{-1}\right.$ $\left.\left(E_{i_{0}}, \ldots, E_{i_{q}}\right)\right)=\eta\left(\left[i_{0} ; E_{i_{0}}, \mathbb{R}, \ldots, \mathbb{R}, E_{i_{1}}, \mathbb{R}, \ldots, \mathbb{R}, E_{i_{q}}\right]\right)=\mu\left(\theta^{-1}\left(\left[i_{0} ; E_{i_{0}}, \mathbb{R}, \ldots, \mathbb{R}, E_{i_{1}}, \mathbb{R}, \ldots, \mathbb{R}, E_{i_{q}}\right]\right)\right)$ $=\mu\left(\varphi_{i_{0}}^{-1}\left(E_{i_{0}}\right) \cap \ldots \cap \varphi_{i_{q}}^{-1}\left(E_{i_{0}}\right)\right)=\mu\left(\left(\varphi_{i_{0}}, \ldots, \varphi_{i_{q}}\right)^{-1}\left(E_{i_{0}}, \ldots, E_{i_{q}}\right)\right)$.

Property (b) implies that $(\sigma, \eta)$, equipped with the observable $\psi$, inherits from $\left(\varphi_{n}\right)_{n \geq 0}$ not only the same distributions (i.e., $\psi \circ \sigma^{n}$ and $\varphi_{n}$ are equally distributed, for all $n \geq 0$ ) but also the same "dependence" (to be understood as the equality of joint distributions). For first consequence of property (b), it suffices to consider a single arbitrary non-negative integer $i_{0}$. 
Considering that $\left(\varphi_{n}\right)_{n \geq 0}$ is IID, property (b) also implies that the family $\left(\psi \circ \sigma^{n}\right)_{n \geq 0}$ is IID.

For identical distribution, $\left(\psi \circ \sigma^{n}\right)_{\star} \eta=\varphi_{n_{*}} \mu=\varphi_{0 *} \mu, \forall n \geq 0$. For independence, let $i_{0}<\ldots<i_{q}$ be arbitrary non-negative integers and $E_{i_{0}}, \ldots, E_{i_{q}}$ be arbitrary measurable sets on the real line. From (b), $\eta\left(\left(\psi \circ \sigma^{i_{0}}\right)^{-1}\left(E_{i_{0}}\right) \cap \ldots \cap\left(\psi \circ \sigma^{i_{q}}\right)^{-1}\left(E_{i_{q}}\right)\right)=\mu\left(\left(\varphi_{i_{0}}, \ldots, \varphi_{i_{q}}\right)^{-1}\left(\left(E_{i_{0}}, \ldots, E_{i_{q}}\right)\right)=\mu\left(\varphi_{i_{0}}^{-1}\left(E_{i_{0}}\right) \cap\right.\right.$ $\left.\ldots \cap \varphi_{i_{q}}^{-1}\left(E_{i_{q}}\right)\right)=\mu\left(\varphi_{i_{0}}^{-1}\left(E_{i_{0}}\right)\right) \ldots \mu\left(\varphi_{i_{q}}^{-1}\left(E_{i_{q}}\right)\right)=\eta\left(\varphi_{i_{0}}^{-1}\left(E_{i_{0}}\right)\right) \ldots \eta\left(\varphi_{i_{q}}^{-1}\left(E_{i_{q}}\right)\right)$. Alternatively, this can be checked directly from $\eta=\nu^{\mathbb{N}}$.

Summing up, we showed two things in a row. First, IID stochastic processes are modeled by Bernoulli shifts (with a projection on the first coordinate). Second, examples of IID Bernoulli shifts. Adapting arguments, it is easy to verify that every Bernoulli shift is IID (with respect to a projection on the first coordinate). These concepts are interconnected.

Note that modeling a stochastic process with dynamical system and an observable enables bringing knowledge from ergodic theory to probability theory. On the other hand, approaching a dynamical system with an observable as a stochastic process enables bringing knowledge from probability theory to ergodic theory. These approaches are interconnected.

As we present next, not every IID system is ergodic. In particular, not every IID system is a Bernoulli system (they still have a Bernoulli factor, though).

Example 4.1.5 (Non ergodic IID system). Let $X=\{0,1\}^{\mathbb{N}} \times\{a, b\}$ and $f: X \rightarrow X$ be given by $\left(\left(x_{0}, x_{1}, \ldots\right), j\right) \mapsto\left(\left(x_{1}, x_{2}, \ldots\right), j\right), j=a, b$. This dynamical system is a sort of duplicated shift, where each separate copy, $\{0,1\}^{\mathbb{N}} \times\{a\}$ and $\{0,1\}^{\mathbb{N}} \times\{b\}$, is identified by the append $a$ or $b$ and is invariant.

The set $X$ is equipped with the topology and $\sigma$-algebra already used for $\{0,1\}^{\mathbb{N}}$ product the discrete topology and $\sigma$-algebra of $\{a, b\}$.

The invariant measure used is $\tilde{\beta}_{p}=0.5 \beta_{p}^{a \mathbb{N}}+0.5 \beta_{p}^{b^{\mathbb{N}}}$, where $\beta_{p}^{j^{\mathbb{N}}}$ (not properly a product measure) is given by: for any $j, k \in\{a, b\}$ and any measurable set $A \subset\{0,1\}^{\mathbb{N}}$, when $j \neq k, \beta_{p}^{j^{\mathbb{N}}}(A \times\{k\})=0$; otherwise, when $j=k, \beta_{p}^{j^{\mathbb{N}}}(A \times\{j\})=\beta_{p}^{\mathbb{N}}(A)$, where $\beta_{p}^{\mathbb{N}}$ on $\{0,1\}^{\mathbb{N}}$ is the product measure of $\beta_{p}$ on $\{0,1\}$, given by $\beta_{p}(\{1\})=p$.

Clearly, $\left(f, \tilde{\beta}_{p}\right)$ is not an ergodic system, since $\{0,1\}^{\mathbb{N}} \times\{a\}$ is invariant and has measure 0.5.

The observable $\psi: X \rightarrow\{0,1\}$, given by $\psi:\left(\left(x_{0}, x_{1}, \ldots\right), j\right) \mapsto x_{0}, j=1,2$, is blind to that duplication.

The argument that $\left(\psi,\left(f, \tilde{\beta}_{p}\right)\right)$ is IID is identical to the previous example. In fact, $\psi$ was designed to not distinguish between the situation in this example and a standard shift, analyzed in the previously.

It is curious that the stochastic process arising from the non-ergodic IID example 4.1 .5 can still be modeled by an ergodic Bernoulli shift, as in example 4.1.4. This Bernoulli shift would be a "non-duplicated mimic" of what is being "perceived" by $\psi$. In some sense, IID systems, although not always ergodic, are still higher (or not under) than Bernoulli systems (in particular, ergodic systems) in "chaos". Proposition 4.1.3 corroborates with this understanding.

We consider the idea of modeling stochastic processes with dynamical systems a bit further. The next example shows it is even possible to consider models for general (non IID) stochastic processes.

Example 4.1.6 (Models for stochastic processes). This example was completely developed within example 4.1.4. Here we just abbreviate the previous analysis to emphasize what can be obtained under more general hypothesis.

Let $(X, \mathcal{A}, \mu)$ be probability space and $\left(\varphi_{n}: X \rightarrow \mathbb{R}\right)_{n \geq 0}$ a stochastic process.

Consider $\theta=\bigotimes_{j=0}^{\infty} \varphi_{n}: X \rightarrow \mathbb{R}^{\mathbb{N}}$ mapping $x \mapsto\left(\varphi_{0}(x), \varphi_{1}(x), \ldots\right)$, the pushforward probability $\eta=\theta_{\star} \mu$, the unilateral shift on $\mathbb{R}^{\mathbb{N}}$ (see example 3.1.9) and the observable $\psi: \mathbb{R}^{\mathbb{N}} \rightarrow \mathbb{R}$ given by $\psi\left(x_{0}, x_{1}, \ldots\right)=x_{0}$.

Different from the last example, $\eta$ will not be a Bernoulli measure. Additionally, at this level of generality, we can not even conclude that $(\sigma, \eta)$ is invariant. Working out the calculations for cylin- 
ders, analogously to the last example, shows that not enough structure is available to conclude invariance: $\eta\left(\sigma^{-1}\left(\left[m ; B_{m}, \ldots, B_{m+k}\right]\right)\right)=\eta\left(\left[m+1 ; B_{m}, \ldots, B_{m+k}\right]\right)=\mu\left(\theta^{-1}\left(\left[m+1 ; B_{m}, \ldots, B_{m+k}\right]\right)\right)=$ $\mu\left(\varphi_{m+1}^{-1}\left(B_{m}\right) \cap \ldots \cap \varphi_{m+1+k}^{-1}\left(B_{m+k}\right)\right)$ not necessarily equals $\mu\left(\varphi_{m}^{-1}\left(B_{m}\right) \cap \ldots \cap \varphi_{m+k}^{-1}\left(B_{m+k}\right)\right)$ $=\mu\left(\theta^{-1}\left(\left[m ; B_{m}, \ldots, B_{m+k}\right]\right)\right)=\eta\left(\left[m ; B_{m}, \ldots, B_{m+k}\right]\right)$.

This calculation shows that $\mu$-identical distribution of the $\varphi_{n}$ 's is a necessary condition for the invariance of $(\sigma, \eta)$, as noted by considering $k=0$ and checking that $\mu\left(\varphi_{m+1}^{-1}(E)\right)$ would equal $\mu\left(\varphi_{m}^{-1}(E)\right)$, for any measurable $E$.

However, the same condition is not sufficient for the invariance of that system, as can be illustrated with the following case: take $\varphi_{0}$ and $\varphi_{1}$ being everywhere different but identically distributed (see example following definition 2.3.1) and $\varphi_{n}=\varphi_{0}, n \geq 2$. The family $\left(\varphi_{n}\right)_{n \geq 0}$ is $\mu$-identically distributed but $\eta([0 ; E, E])=\mu\left(\theta^{-1}([0 ; E, E])\right)=\mu\left(\varphi_{0}^{-1}(E) \cap \varphi_{1}^{-1}(E)\right)=0$ while $\mu([2 ; E, E])=$ $\mu\left(\theta^{-1}([2 ; E, E])\right)=\mu\left(\varphi_{2}^{-1}(E) \cap \varphi_{3}^{-1}(E)\right)>0$, where $E$ is an appropriate measurable set. As a consequence, $(\sigma, \eta)$ is not invariant.

As we checked in example 4.1.4, properties (a) and (b) previously introduced are verified regardless of whether $\left(\varphi_{n}\right)_{n \geq 0}$ is IID. Therefore, in the sense of properties (a) and (b), we were capable of presenting a model to the stochastic process $\left(\varphi_{n}\right)_{n \geq 0}$ which is a dynamical system equipped with a non-invariant measure, $(\sigma, \eta)$. The invariance of $(\sigma, \eta)$ is contingent to discussion we just had. In any case, $\eta$ still of interest because, obviously, it makes $\theta$ into a measure preserving transformation.

We have been precise when discussing what should be understood as a model in the context of the previous examples, but little background was provided for this concept. The idea is that a model provides an object that is indistinguishable from another according to a certain point of view. A notion equivalence relation among stochastic processes naturally emerge. We will touch this issue really briefly, just for the reader to be able to wrap his or her head around.

Let $(X, \mathcal{A}, \mu)$ be a probability space and $\left(\varphi_{n}\right)_{n \geq 0}$ and $\left(\psi_{n}\right)_{n \geq 0}$ be two $\mathbb{R}$-valued stochastic processes on $X$. They are said to equivalent in distribution if they have the same finite dimensional distributions, i.e., for any finite collection of non-negative integers $i_{0}<\ldots<i_{q},\left(\varphi_{i_{0}}, \ldots, \varphi_{i_{q}}\right)$ and $\left(\psi_{i_{0}}, \ldots, \psi_{i_{q}}\right)$ have the same distributions. They are said to be equivalent if $\mu\left(\left\{x \in X: \varphi_{n}(x)=\right.\right.$ $\left.\left.\psi_{n}(x)\right\}\right)=1, \forall n \geq 0$. In particular, since the indexing set $\Lambda=\mathbb{N}$ is countable, $\mu\left(\cap_{n \in \mathbb{N}}\left\{x \in X: \varphi_{n}(x)=\right.\right.$ $\left.\left.\psi_{n}(x)\right\}\right)=\mu\left(\left\{x \in X: \varphi_{n}(x)=\psi_{n}(x), \forall n \geq 0\right\}\right)=1$. Whenever the index set $\Lambda$ is uncountable, this last equality will not hold, so it is used a definition itself, when the stochastic processes are said to be indistinguishable.

The first two definitions above create an equivalence relation on the collection of (discrete) $\mathbb{R}$ valued stochastic processes on $X$. The first definition overlooks random variables being everywhere different but having the same distribution (see example following definition 2.3.1). The second definition fixes this deficiency. Clearly, the second definition is much more restrictive, and implies the first one.

We can consider a generalization of equivalence in distribution to allow for $\mathbb{R}$-valued stochastic processes on different measure spaces. Models for stochastic processes, as presented before, satisfy this notion of equivalence.

In fact, under additional hypothesis on the measure space $(X, \mathcal{A}, \mu)$, a (discrete) $\mathbb{R}$-valued stochastic process on $X$ can be modeled directly by a dynamical system on $X$ (equipped with an observable). We will not go further in this direction.

At this point, we conclude the content of this section. Next we summarize some takeaways and provide the link with the following section.

Up to here, readers were introduced to a joint framework comprising ergodic and probability theories. It was emphasized how dynamical systems (with observables) can be approached as stochastic processes and how stochastic processes can be modeled by dynamical systems (with observables).

This machinery allows, in particular, concepts and results from probability theory to be adapted to the ergodic theory setting. In special, the key concept of IID stochastic processes (or random variables) was adapted to the concept of IID observables with respect to a system and the concept of IID systems. We discussed how IID systems relate with other concepts from ergodic theory. 
The reader should notice the framework and attitude herein presented generalize and are applicable to adapting all of the probabilistic machinery available out there. In the next section we will focus on how to prove and adapt the law of large numbers.

Since the (probabilistic) law of large numbers uses IID random variables as a key assumption, the adapted notion of IID developed in this section will be central to understand what is going to be done in the next section.

\subsection{Law of Large Numbers}

Now we will narrow our efforts to translate the Law of Large Numbers and discuss how this result relates to those already discussed, in special, the Birkhoff's ergodic theorems.

The intuition about the Birkhoff's ergodic theorems should be clear by now. So we will invest some words to discuss the statement and explain the probabilistic intuition of the Law of Large Numbers. We hope both intuitions combine.

Theorem 4.2.1 (Weak law of large numbers). Let $(X, \mathcal{A}, \mu)$ be a probability space and $\left(\psi_{n}: X \rightarrow\right.$ $\mathbb{R})_{n \geq 0}$ be an IID sequence of random variables such that $\int \psi_{0} d \mu \in \mathbb{R}$ and $\operatorname{var}\left(\psi_{0}\right) \in \mathbb{R}$.

Then the sequence of $\mathbb{R}$-valued random variables on $X,\left(\frac{\psi_{0}+\ldots+\psi_{n-1}}{n}\right)_{n \geq 1}$, satisfies $\lim _{n} \frac{\psi_{0}+\ldots+\psi_{n-1}}{n}=$ $\int \psi_{0} d \mu, \mu-a . e .$.

Theorem 4.2.2 (Strong law of large numbers). Let $(X, \mathcal{A}, \mu)$ be a probability space and $\left(\psi_{n}: X \rightarrow\right.$ $\mathbb{R})_{n \geq 0}$ be an IID sequence of random variables such that $\int \psi_{0} d \mu \in \overline{\mathbb{R}}( \pm \infty$ allowed).

Then the sequence of $\mathbb{R}$-valued random variables on $X,\left(\frac{\psi_{0}+\ldots+\psi_{n-1}}{n}\right)_{n \geq 1}$, satisfies $\lim _{n} \frac{\psi_{0}+\ldots+\psi_{n-1}}{n}=$ $\int \psi_{0} d \mu, \mu$-a.e.

The idea is to consider an IID sequence of random variables, $\psi_{n}$ 's, and face their running averages, $\frac{\psi_{0}+\ldots+\psi_{n-1}}{n}$ 's, as random variables. Intuitively, these $\frac{\psi_{0}+\ldots+\psi_{n-1}}{n}$ 's are probabilistic representations of what should be the averaged result of $n$ IID draws from a distribution equal to that of the $\psi$ 's. The theorem states that this average will, with probability 1 , approach the integral of the $\psi$ 's.

The first part of the proof of theorem 4.2.1, to be presented, gives a fairly quick and useful reason to be comfortable with the conclusion: the integral of the running average of the $\psi_{n}$ 's is constant at $\int \psi_{0} d \mu$, while their variance shrinks to 0 as $n$ increases. That is not a proof of a.e. convergence, but it is a starting point to approach to result.

Instead of the sequence $A(\psi)_{n}(x)=\frac{\psi_{0}(x)+\ldots+\psi_{n-1}(x)}{n}, n \geq 0$, of $\mathbb{R}$-valued random variables on $(X, \mathcal{A}, \mu)$, one could consider the sequence $B(\psi)_{n}(\hat{x})=B(\psi)_{n}\left(x_{0}, x_{1}, \ldots, x_{n-1}, \ldots\right)=\frac{\psi_{0}\left(x_{0}\right)+\ldots+\psi_{n-1}\left(x_{n-1}\right)}{n}$ of $\mathbb{R}$-valued random variables on $\left(X^{\mathbb{N}}, \mathcal{A}^{\mathbb{N}}, \mu^{\mathbb{N}}\right)$. The second sequence explicitly supports the interpretation on the last paragraph, once each of the $n$ IID draws is separately indicated with $x_{0}, x_{1}, \ldots$

We will discuss how these sequences relate. For such, define $\varphi_{n}: X^{\mathbb{N}} \rightarrow \mathbb{R}$ given by $\varphi_{n}(\hat{x})=$ $\varphi_{n}\left(x_{0}, x_{1}, \ldots, x_{n-1}, \ldots\right)=\psi_{n}\left(x_{n}\right)$. In this case, $B(\psi)_{n}(\hat{x})=\frac{\varphi_{0}(\hat{x})+\ldots \varphi_{n-1}(\hat{x})}{n}\left(=A(\varphi)_{n}\right.$, if $A$ is to be understood broadly).

Note that the $\varphi_{n}$ 's are also IID. For identical distribution, we show that each $\varphi_{n}$ and $\psi_{n}$ is identically distributed, once $\mu^{\mathbb{N}}\left(\varphi_{n}^{-1}(A)\right)=\mu^{\mathbb{N}}\left(\left[n ; \psi_{n}^{-1}(A)\right]\right)=\mu\left(\psi_{n}^{-1}(A)\right)$, for any measurable $A \subset \mathbb{R}$. For independence, it is enough to check that for any non-negative integers $i_{0}<\ldots<$ $i_{q}$ and measurable sets on the real line $E_{0}, \ldots, E_{q}$, we have $\mu^{\mathbb{N}}\left(\left(\varphi_{i_{0}}, \ldots, \varphi_{i_{q}}\right)^{-1}\left(E_{0}, \ldots, E_{q}\right)\right)=$ $\mu^{\mathbb{N}}\left(\left[i_{0}, \psi_{i_{0}}^{-1}\left(E_{0}\right), X, \ldots, X, \psi_{i_{q}}^{-1}\left(E_{q}\right)\right]\right)=\mu\left(\psi_{i_{0}}^{-1}\left(E_{0}\right)\right) \ldots \mu\left(\psi_{i_{q}}^{-1}\left(E_{q}\right)\right)=\mu^{\mathbb{N}}\left(\varphi_{i_{0}}^{-1}\left(E_{0}\right)\right) \ldots \mu^{\mathbb{N}}\left(\varphi_{i_{q}}^{-1}\left(E_{q}\right)\right)$.

More generally, we can check that the finite distributions of the $\psi_{n}$ 's and the $\varphi_{n}$ 's coincide. Choosing any non-negative integers $i_{0}<\ldots<i_{q}$ and measurable sets on the real line $E_{0}, \ldots, E_{q}$, we have $\mu^{\mathbb{N}}\left(\left(\varphi_{i_{0}}, \ldots, \varphi_{i_{q}}\right)^{-1}\left(E_{0}, \ldots, E_{q}\right)\right)=\mu^{\mathbb{N}}\left(\left[i_{0}, \psi_{i_{0}}^{-1}\left(E_{0}\right), X, \ldots, X, \psi_{i_{q}}^{-1}\left(E_{q}\right)\right]\right)=\mu\left(\psi_{i_{0}}^{-1}\left(E_{0}\right)\right) \ldots$ $\mu\left(\psi_{i_{q}}^{-1}\left(E_{q}\right)\right)=\mu\left(\left(\psi_{i_{0}}, \ldots, \psi_{i_{q}}\right)^{-1}\left(E_{0}, \ldots, E_{q}\right)\right)$.

Therefore, $A(\psi)_{n}$ and $B(\psi)_{n}$ are equivalent in distribution. Additionally, the measure preserving $n$-th coordinate projection, $\pi_{n}: X^{\mathbb{N}} \rightarrow X, n \geq 0$, makes the associated diagram to commute as $B(\psi)_{n}(\hat{x})=A(\psi)_{n}\left(\pi_{n}(\hat{x})\right)$. 
Since the $\varphi_{n}$ 's are IID, theorem 4.2.2 is again applicable. It concludes that the running averages of the $\varphi_{n}$ 's, namely, the $B(\psi)_{n}$ 's, converge a.e.: $\frac{\varphi_{0}(\hat{x})+\ldots \varphi_{n-1}(\hat{x})}{n}=\frac{\psi_{0}\left(x_{0}\right)+\ldots+\varphi_{n-1}\left(x_{n-1}\right)}{n} \rightarrow \int \varphi_{0} d \mu^{\mathbb{N}}=$ $\int \psi_{0} d \mu, \mu^{\mathbb{N}}$-a.e. $\hat{x} \in X^{\mathbb{N}}$.

Therefore, starting with $\left(\psi_{n}\right)_{n \geq 0}$ IID, theorem 4.2 .2 implies not only that $\frac{\psi_{0}(x), \ldots \psi_{n-1}(x)}{n} \rightarrow$ $\int \psi_{0} d \mu, \mu$-a.e., but also that $\frac{\psi_{0}\left(x_{0}\right)+\ldots+\psi_{n-1}\left(x_{n-1}\right)}{n} \rightarrow \int \psi_{0} d \mu, \mu^{\mathbb{N}}$-a.e., wrapping up our interpretation of its meaning.

As a matter of curiosity, the sequence of the $\frac{\psi_{1}+\ldots+\psi_{n}}{n}$ 's is not IID, thus not a candidate for a subsequent application of theorem 4.2.2. However, as theorem 4.2.2 already guarantees a.e. convergence, taking another sequence of running averages upon the $\frac{\psi_{1}+\ldots+\psi_{n}}{n}$ 's adds nothing: they will still converge to the same value.

Now we restate and prove both theorems 4.2.1 and 4.2.2, adapting their claims and arguments to an ergodic theory language. In essence, these proofs do not differ from standard probabilistic proofs. The next two proofs were adapted from [Çın11].

Theorem 4.2.3 (Weak law of large numbers, adapted). Let $(X, \mathcal{A})$ be a measurable space, $f: X \rightarrow$ $X$ be a measurable dynamical system and a $\mu$ be probability measure on $(X, \mathcal{A})$ such that $(f, \mu)$ is an invariant system.

Then $\forall \psi \in L^{2}(\mu, \mathbb{R})$ such that $(\psi,(f, \mu))$ is IID, $\exists X_{\psi} \in \mathcal{A}, \mu\left(X_{\psi}\right)=1$, such that $\frac{1}{n} \sum_{j=0}^{n-1} \psi \circ$ $f^{j}(x) \rightarrow \int \psi d \mu, \forall x \in X_{\psi}$.

Proof. Since $\left(\psi \circ f^{n}\right)_{n \geq 0}$ is identically distributed, proposition 2.3 .10 , guarantees that $\int \psi \circ f^{n} d \mu=$ $\int \psi d \mu=: a, \forall n \geq 0$ and $\operatorname{var}\left(\psi \circ f^{n}\right)=\operatorname{var}(\psi)=: b, \forall n \geq 0$.

The average and variance for the sequence of random variables $\left(1 / n \sum_{j=0}^{n-1} \psi \circ f^{n}\right)_{n \geq 0}$ is easily determined: (I) $\int \frac{1}{n} \sum_{j=0}^{n-1} \psi \circ f^{j} d \mu=\frac{1}{n} \sum_{j=0}^{n-1} \int \psi \circ f^{j} d \mu=\frac{1}{n} n a=a,(\mathrm{II}) \operatorname{var}\left(\frac{1}{n} \sum_{j=0}^{n-1} \psi \circ f^{j}\right) \stackrel{(*)}{=}$ $\frac{1}{n^{2}} \operatorname{var}\left(\sum_{j=0}^{n-1} \psi \circ f^{j}\right) \stackrel{(*)}{=} \frac{1}{n^{2}} \sum_{j=0}^{n-1} \operatorname{var}\left(\psi \circ f^{j}\right)=\frac{1}{n^{2}} n b=\frac{b}{n}$. For $(*)$ and $(* *)$, see discussion after proposition 2.3.10.

For the time being, note that the average of the $1 / n \sum_{j=0}^{n-1} \psi \circ f^{j}$ 's is consistently $a$ while their variance, $b / n$, is shrinking as $n$ increases. This is a good sign, but not yet close to $\mu$-a.e. convergence. We will first obtain $\mu$-a.e. convergence through a subsequence.

First note that by the Chebyshev inequality, proposition 2.3.12, $\mu\left(\left\{x \in X: \mid 1 / n \sum_{j=0}^{n-1} \psi \circ f^{j}(x)-\right.\right.$ $a \mid>\epsilon\}) \leq \operatorname{var}\left(1 / n \sum_{j=0}^{n-1} \psi \circ f^{j}\right) / \epsilon^{2}=\frac{b}{\epsilon^{2} n}$.

Now we consider the subsequence $n_{k}=k^{2}, k \geq 1$, and, with $\alpha_{n}=1 / n \sum_{j=0}^{n-1} \psi \circ f^{j}$, define $\varphi_{k}=$ $\alpha_{n_{k}}=\alpha_{k^{2}}$, in order to apply proposition 2.3.15-(i) as follows: $\mu\left(\left\{x \in X:\left|\varphi_{k}(x)-a\right|>\epsilon\right\}\right)=\mu(\{x \epsilon$ $\left.\left.X:\left|1 / k^{2} \sum_{j=0}^{k^{2}-1} \psi \circ f^{j}(x)-a\right|>\epsilon\right\}\right) \leq \frac{b}{\epsilon^{2} k^{2}}$ and, summing up across $k, \sum_{k=1}^{\infty} \mu\left(\left\{x \in X:\left|\varphi_{k}(x)-a\right|>\right.\right.$ $\epsilon\}) \leq \frac{b}{\epsilon^{2}} \sum_{k=0}^{\infty} 1 / k^{2}<\infty$. Since $\epsilon$ was arbitrary, proposition 2.3.15(i) guarantees that $\varphi_{k} \stackrel{k}{\rightarrow} a$, $\mu$-a.e.. In other words, $1 / n_{k} \sum_{j=0}^{n_{k}-1} \psi \circ f^{j} \stackrel{k}{\rightarrow} a$, $\mu$-a.e. Denote the set where this convergence occurs by $X_{0}$.

Suppose for a while that $\psi \circ f^{n} \geq 0, \forall n \geq 0$. This allows us to use the following real analysis lemma.

Lemma. Let $\left(x_{n}\right)_{n \geq 0}$ be a sequence of non-negative real numbers, $\bar{x}_{n}=\frac{x_{0}+\ldots+x_{n-1}}{n}$ and $\left(n_{k}\right)_{k \geq 1}$ be an increasing subsequence of natural numbers such that $\lim _{k} n_{k+1} / n_{k}=r$ and $\lim _{k} \bar{x}_{n_{k}}=x$, then $x / r \leq \liminf _{n} \bar{x}_{n} \leq \lim \sup _{n} \bar{x}_{n} \leq r x$.

Proof. For a given $n$, consider $k(n)$ the largest $k$ such that $n_{k} \leq n$. Hence $n_{k(n)} \leq n \leq n_{k(n)+1}$. Then $\frac{n_{k(n)}}{n_{k(n)}} \frac{x_{0}+\ldots+x_{n k(n)^{-1}}}{n_{k(n)+1}} \leq \frac{x_{0}+\ldots+x_{n-1}}{n} \leq \frac{x_{0}+\ldots+x_{n_{k(n)+1}-1}}{n_{k(n)}} \frac{n_{k(n)+1}}{n_{k(n)+1}}$, by simple comparison of numerators (the $x_{n}$ 's are positive) and denominators, separately. As consequence, $\frac{n_{k(n)}}{n_{k(n)+1}} \bar{x}_{n_{k(n)}} \leq \bar{x}_{n} \leq \bar{x}_{n_{k(n)+1}} \frac{n_{k(n)+1}}{n_{k(n)}}$.

When $n$ goes to infinity, so does $k(n)$, and those fractions go to $1 / r$ and $r$, respectively, while the $\bar{x}$ 's on the left and right go to $x$. 
For each $x \in X_{0}$, since the partial averages of the non-negative sequence $\left(\psi \circ f^{j}(x)\right)_{n \geq 0}$ converges to $a$ along $n_{k}=k^{2}$ and $r:=\lim _{k} \frac{n_{k+1}}{n_{k}}=\lim _{k} \frac{(k+1)^{2}}{k^{2}}=1$, the lemma shows that $a \leq \liminf _{n} \frac{1}{n} \sum_{j=0}^{n-1} \psi$ 。 $f^{j}(x) \leq \lim \sup _{n} \frac{1}{n} \sum_{j=0}^{n-1} \psi \circ f^{j}(x) \leq a$. Therefore, $\frac{1}{n} \sum_{j=0}^{n-1} \psi \circ f^{j}(x) \rightarrow a=\int \psi d \mu$, $\mu$-a.e. $x \in X$.

If it was not the case that $\psi \circ f^{n} \geq 0, \forall n \geq 0$, define $\varphi^{+}:=\max \{0, \varphi\}, \varphi^{-}:=\max \{0,-\varphi\}$, such that $\varphi=\varphi^{+}-\varphi^{-}$. Note that $\left(\psi \circ f^{n}\right)^{ \pm}=\psi^{ \pm} \circ f^{n}$. Both $\psi^{+} \circ f^{n}$ and $\psi^{-} \circ f^{n}$ are positive and the conclusion above holds: (i) $\frac{1}{n} \sum_{j=0}^{n-1} \psi^{+} \circ f^{j} \rightarrow \int \psi^{+} d \mu, \forall x \in X_{1}$, where $\mu\left(X_{1}\right)=1$; (ii) $\frac{1}{n} \sum_{j=0}^{n-1} \psi^{-} \circ f^{j} \rightarrow \int \psi^{-} d \mu, \forall x \in X_{2}$, where $\mu\left(X_{2}\right)=1$. Taking (i) minus (ii): $\frac{1}{n} \sum_{j=0}^{n-1} \psi \circ f^{j}(x)=$ $\frac{1}{n} \sum_{j=0}^{n-1}\left(\psi^{+}-\psi^{-}\right) \circ f^{j}(x) \rightarrow \int \psi^{+}-\psi^{+} d \mu=\int \psi d \mu, \forall x \in X_{0}=X_{1} \cap X_{2}$, with $\mu\left(X_{0}\right)=1$.

Theorem 4.2.4 (Strong law of large numbers, adapted). Let $(X, \mathcal{A})$ be a measurable space, $f$ : $X \rightarrow X$ be a measurable dynamical system and a $\mu$ be probability measure on $(X, \mathcal{A})$ such that $(f, \mu)$ is an invariant system.

Then for all measurable $\psi: X \rightarrow \mathbb{R}$, with $\int \psi d \mu \in \overline{\mathbb{R}}( \pm \infty$ allowed), such that $(\psi,(f, \mu))$ is IID, $\exists X_{\psi} \in \mathcal{A}, \mu\left(X_{\psi}\right)=1$, such that $\frac{1}{n} \sum_{j=0}^{n-1} \psi \circ f^{j}(x) \rightarrow \int \psi d \mu, \forall x \in X_{\psi}$.

Proof. Since $\left(\psi \circ f^{n}\right)_{n \geq 0}$ is identically distributed, proposition 2.3.10, guarantees that $\int \psi \circ f^{n} d \mu=$ $\int \psi d \mu=: a, \forall n \geq 0$ and $\operatorname{var}\left(\psi \circ f^{n}\right)=\operatorname{var}(\psi)=: b, \forall n \geq 0$. Note $\operatorname{var}(\cdot)$ is always defined in $[0, \infty]$, so $b$ can be valued $+\infty$. As already disclaimed, $a$ can also be valued $\pm \infty$.

The easy case is when $\int \psi d \mu=\infty$. In this case $\int \psi^{+} d \mu=\infty$ and $\int \psi^{-} d \mu \in \mathbb{R}$, where $\psi^{+}=$ $\max \{\psi, 0\}$ and $\psi^{-}=\max \{-\psi, 0\}$.

Fix $b>0$ and define the truncation $\varphi_{n}=\min \left\{\psi \circ f^{n}, b\right\}=\min \{\psi, b\} \circ f^{n}$. Since independence is preserved after measurable composition (see discussion after proposition 2.3.8), the family $\left(\varphi_{n}\right)_{n \geq 0}$ is independent. The family $\left(\varphi_{n}\right)_{n \geq 0}$ is also identically distributed, as it can be verified: if $h<b$, $\mu\left(\varphi_{n}^{-1}((-\infty, h])\right)=\mu\left(\left(\psi \circ f^{n}\right)^{-1}((-\infty, h])\right)=: u, \forall n \geq 0$; if $h \geq b, \mu\left(\varphi_{n}^{-1}((-\infty, h])\right)=\mu(X), \forall n \geq 0$.

Once the family $\left(\varphi_{n}\right)_{n \geq 0}$ is bounded (hence $\left.\int \varphi_{0} d \mu<\infty\right)$, theorem 4.2 .3 applies. Therefore, $\frac{1}{n} \sum_{j=0}^{n-1} \varphi_{j}(x)=\frac{1}{n} \sum_{j=0}^{n-1} \min \{\psi, b\} \circ f^{j}(x) \rightarrow \int \varphi_{0} d \mu=\int \min \{\psi, b\} d \mu, \forall x \in X_{0}$, where $\mu\left(X_{0}\right)=1$.

Since $\psi \circ f^{n} \geq \varphi_{n}, \forall n \geq 0$, then for each $x \in X_{0}: \liminf \frac{1}{n} \sum_{j=0}^{n-1} \psi \circ f^{j}(x) \geq \liminf \frac{1}{n} \sum_{j=0}^{n-1} \varphi_{j}(x)=$ $\lim \frac{1}{n} \sum_{j=0}^{n-1} \varphi_{j}(x)=\int \min \{\psi, b\} d \mu$.

Since $b>0$ is arbitrary, we can take as large as we want. Also note that: $\lim _{b \rightarrow \infty} \int \min \{\psi, b\} d \mu=$ $\int \psi d \mu=\infty$, by the monotone convergence theorem (see 2.1.21). Therefore, by fixing appropriate $b$, we can find $\lim \inf 1 / n \sum_{j=0}^{n-1} \psi \circ f^{j}$ to be arbitrarily large in a set of full measure.

We state that $\liminf 1 / n \sum_{j=0}^{n-1} \psi \circ f^{j}(x)=\infty, \mu$-a.e. $x \in X$. Therefore $\lim 1 / n \sum_{j=0}^{n-1} \psi \circ f^{j}=\infty, \mu$ a.e. $x \in X$, as desired. If the first statement was not true, there would exist a measurable set $A, \mu(A)>$ 0 , such that $0 \leq \liminf 1 / n \sum_{j=0}^{n-1} \psi \circ f^{j}(x) \neq \infty, \forall x \in A$. Defining $A_{i}=\left\{x \in A: \liminf 1 / n \sum_{j=0}^{n-1} \psi \circ\right.$ $\left.f^{j}(x) \in[i-1, i)\right\}$, we can write $A=\dot{\cup}_{i \geq 1} A_{i}$, then we should have $\mu\left(A_{i^{*}}\right)>0$, for some $i^{*} \geq 1$. Take $K>i^{*}$. As we know, by choosing $b$ large enough, $\liminf 1 / n \sum_{j=0}^{n-1} \psi \circ f^{j} \geq K$ in a set of full measure, what contradicts being $\mu\left(A_{i^{*}}\right)>0$.

The case in which $\int \psi d \mu=-\infty$ is treated analogously.

If $\int \psi d \mu \in \mathbb{R}$, we start assuming $\psi \circ f^{n} \geq 3, \forall n \geq 0$.

Define the truncation $\varphi_{n}=\psi \circ f^{n} \mathcal{X}_{\left\{\psi \circ f^{n}<n\right\}}$, where $\mathcal{X}_{\left\{\psi \circ f^{n}<n\right\}}$ is the characteristic function of the set $\left\{x \in X: \psi \circ f^{n}(x)<n\right\}$.

Note that $\sum_{n=0}^{\infty} \mu\left(\left\{x \in X: \psi \circ f^{n}(x) \neq \varphi_{n}(x)\right\}\right)=\sum_{n=0}^{\infty} \mu\left(\left\{x \in X: \psi \circ f^{n}(x) \geq n\right\}\right)=\sum_{n=0}^{\infty} \mu(\{x \in$ $X: \psi(x) \geq n\})$, since $\psi \circ f^{n}$ and $\psi$ are identically distributed and $\mu\left(\left(\psi \circ f^{n}\right)^{-1}([n, \infty))\right)=$ $\mu\left(\psi^{-1}([n, \infty))\right)$. Additionally, $\sum_{n=0}^{\infty} \mu(\{x \in X: \psi(x) \geq n\}) \leq \int_{0}^{\infty} \mu(\{x \in X: \psi(x) \geq s\}) d s=\int \psi d \mu<$ $\infty$, where the last equality due to proposition 2.3.9.

Since $\sum_{n=0}^{\infty} \mu\left(\left\{x \in X: \psi \circ f^{n}(x) \neq \varphi_{n}(x)\right\}\right)<\infty$, by proposition 2.3.14-(i), $\sum_{n=0}^{\infty} \mathcal{X}_{\left\{x \in X: \psi \circ f^{n}(x) \neq \varphi_{n}(x)\right\}}$ $<\infty, \mu$-a.e.. Let $X_{0}$ be the set of full measure where the last characterization holds. So, for every $x \in X_{0}, \psi \circ f^{n}(x)=\varphi_{n}(x)$ for all but finitely many $n$ 's.

Since when $x \in X_{0}, \psi \circ f^{n}(x)$ and $\varphi_{n}(x)$ coincide for all but finitely many $n$ 's, their averages are $\epsilon$-close whenever $n$ is large enough, in particular, their long term behavior coincide, i.e., $\lim _{n} 1 / n \sum_{j=0}^{n-1} \psi \circ f^{j}(x)$ exists and equals $\lim _{n} 1 / n \sum_{j=0}^{n-1} \varphi_{j}(x)$ whenever the later term is well defined. 
Therefore it suffices to prove that $1 / n \sum_{j=0}^{n-1} \varphi_{j}(x) \rightarrow \int \psi d \mu, \forall x \in X_{1}$, where $\mu\left(X_{1}\right)=1$. After that, it is enough to consider $X_{\psi}=X_{0} \cap X_{1}$ as the set of full measure where $1 / n \sum_{j=0}^{n-1} \psi \circ f^{j}(x) \rightarrow \int \psi d \mu$, concluding the argument.

Since independence is preserved after measurable composition (see discussion after proposition 2.3.8), the family $\left(\varphi_{n}\right)_{n \geq 0}$ is independent. Each $\varphi_{n}=\psi \circ f^{n} \mathcal{X}_{\left\{\psi \circ f^{n}<n\right\}}$ is also identically distributed to $\psi \mathcal{X}_{\{\psi<n\}}$, as it can be verified: let $n$ be an arbitrary non-negative integer, if $0<$ $h<n, \mu\left(\varphi_{n}^{-1}((0, h])\right)=\mu\left(\left(\psi \circ f^{n}\right)^{-1}((0, h])\right)=\mu\left(\psi^{-1}((0, h])\right)=\mu\left(\left(\psi \mathcal{X}_{\{\psi<n\}}\right)^{-1}((0, h])\right)$; if $n \leq h$, $\mu\left(\varphi_{n}^{-1}((0, h])\right)=\mu\left(\left(\psi \circ f^{n}\right)^{-1}((0, n))\right)=\mu\left(\psi^{-1}((0, n))\right)=\mu\left(\left(\psi \mathcal{X}_{\{\psi<n\}}\right)^{-1}((0, h])\right)$; and $\mu\left(\varphi_{n}^{-1}(\{0\})\right)=$ $\left.\mu\left(\left(\psi \circ f^{n}\right)^{-1}[n, \infty)\right)\right)=\mu\left(\psi^{-1}([n, \infty))\right)=\mu\left(\left(\psi \mathcal{X}_{\{\psi<n\}}\right)^{-1}(\{0\})\right)$.

Hence, $\int \sum_{j=0}^{n-1} \varphi_{j} d \mu=\sum_{j=0}^{n-1} \int \varphi_{j} d \mu=\sum_{j=0}^{n-1} \int \psi \mathcal{X}_{\{\psi<j\}} d \mu=\int \sum_{j=0}^{n-1} \psi \mathcal{X}_{\{\psi<j\}} d \mu=\int \psi \sum_{j=0}^{n-1} \mathcal{X}_{\{\psi<j\}} d \mu$ $=\int \psi \sum_{j>\psi} \delta_{j}([0, n-1]) d \mu$, where $\sum_{j=0}^{n-1} \mathcal{X}_{\{\psi<j\}}=\sum_{j>\psi} \delta_{j}([0, n-1])=: \lambda_{n}$, being the first function the characteristic of the set $\{x \in X: \psi(x)<j\}$ and the second that mapping $x \mapsto \sum_{j>\psi(x)} \delta_{j}([0, n-1])$, both counting the positive integers between $\psi(x)$ (exclusive) and $n-1$ (inclusive). Therefore, $\lim _{n} \int 1 / n \sum_{j=0}^{n-1} \varphi_{j} d \mu=\lim _{n} \int \psi \frac{\lambda_{n}}{n} d \mu=\int \psi d \mu$, where the last inequality holds because $\frac{\lambda_{n}}{n} \rightarrow 1$ pointwise and $\psi \frac{\lambda_{n}}{n}$ is dominated by $\psi$ as long as $\frac{\lambda_{n}}{n} \leq 1$, allowing the use of the dominated convergence theorem (see 2.1.26). So at least the integral $\int 1 / n \sum_{j=0}^{n-1} \varphi_{j} d \mu$ goes to $\int \psi d \mu$, a good first sign.

We will now prove $1 / n \sum_{j=0}^{n-1} \varphi_{j} \rightarrow \int \psi d \mu, \mu$-a.e. In fact, that $\mu$-a.e. convergence will be proved along a convenient subsequence, $\left(n_{k}\right)_{k \geq 1}$, which is enough. Even better, to obtain this $\mu$-a.e. convergence along a subsequence, since $\int 1 / n \sum_{j=0}^{n-1} \varphi_{j} d \mu \rightarrow \int \psi d \mu$, as we just proved, it suffices to check that $1 / n \sum_{j=0}^{n-1} \varphi_{j}-\int 1 / n \sum_{j=0}^{n-1} \varphi_{j} d \mu \rightarrow 0$, along $\left(n_{k}\right)_{k \geq 0}, \mu$-a.e.. After checking this last fact, we will refresh the reader as to why that a $\mu$-a.e. convergence along a convenient subsequence suffices.

Define the subsequence $\left(n_{k}\right)_{k \geq 1}$ as a function of a parameter $a>0:\left(n_{k}\right)_{k \geq 1}=\left(n_{k}^{a}\right)_{k \geq 1}$, where $n_{k}^{a}$ is the smallest integer strictly greater than $e^{a k}$.

We will show that $1 / n \sum_{j=0}^{n-1} \varphi_{j}-\int 1 / n \sum_{j=0}^{n-1} \varphi_{j} d \mu \rightarrow 0, \mu$-a.e. along $\left(n_{k}\right)_{k \geq 0}$ by using proposition 2.3.15-(i): let $\theta_{k}=1 / n_{k} \sum_{j=0}^{n_{k}-1} \varphi_{n_{k}}$ and verify that $\sum_{k=1}^{\infty} \mu\left(\left\{x \in X:\left|\theta_{k}-\int \theta_{k} d \mu\right|>\epsilon\right\}\right)=\sum_{k=0}^{\infty} \mu(\{x \epsilon$ $\left.\left.X:\left|1 / n_{k} \sum_{j=0}^{n_{k}-1} \varphi_{j}(x)-\int 1 / n_{k} \sum_{j=0}^{n_{k}-1} \varphi_{j} d \mu\right|>\epsilon\right\}\right)<\infty, \forall \epsilon>0$. We will justify the last inequality in a while. Beforehand, see that proposition 2.3.15-(i) guarantees that $\theta_{k}-\int \theta_{k} d \mu \rightarrow 0, \mu$-a.e., implying $1 / n_{k} \sum_{j=0}^{n_{k}-1} \varphi_{n_{k}}-\int 1 / n_{k} \sum_{j=0}^{n_{k}-1} \varphi_{n_{k}} d \mu \rightarrow 0, \mu$-a.e..

Now we work to justify the last inequality. Start using Chebyshev's inequality (see 2.3.12): $\epsilon^{2} \mu\left(\left\{x \in X:\left|1 / n \sum_{j=0}^{n-1} \varphi_{j}(x)-\int 1 / n \sum_{j=0}^{n-1} \varphi_{j} d \mu\right|>\epsilon\right\}\right) \leq \operatorname{var}\left(1 / n \sum_{j=0}^{n-1} \varphi_{j}\right), \forall n \geq 1$. Summing across our subsequence: $\epsilon^{2} \sum_{k=1}^{\infty} \mu\left(\left\{x \in X:\left|1 / n_{k} \sum_{j=0}^{n_{k}-1} \varphi_{j}(x)-\int 1 / n_{k} \sum_{j=0}^{n_{k}-1} \varphi_{j} d \mu\right|>\epsilon\right\}\right) \leq \sum_{k=1}^{\infty} \operatorname{var}\left(1 / n_{k} \sum_{j=0}^{n_{k}-1} \varphi_{j}\right)$ $\stackrel{(*)}{=} \sum_{k=1}^{\infty} 1 / n_{k}^{2} \sum_{j=0}^{n_{k}-1} \operatorname{var}\left(\varphi_{j}\right)$, where $(*)$ is due to homogeneity of degree two of the variance and its linearity under the independence of the $\varphi_{n}$ 's (see discussion after proposition 2.3.10).

Additionally, $\sum_{j=0}^{n-1} \operatorname{var}\left(\varphi_{j}\right)=\sum_{j=0}^{n-1} \int \varphi_{j}^{2} d \mu-\left(\int \varphi_{j} d \mu\right)^{2} \leq \sum_{j=0}^{n-1} \int \varphi_{j}^{2} d \mu \stackrel{(*)}{=} \sum_{j=0}^{n-1} \int\left(\psi \mathcal{X}_{\{\psi<j\}}\right)^{2} d \mu=$ $\sum_{j=0}^{n-1} \int \psi^{2}\left(\mathcal{X}_{\{\psi<j\}}\right)^{2} d \mu=\sum_{j=0}^{n-1} \int \psi^{2} \mathcal{X}_{\{\psi<j\}} d \mu=\int \sum_{j=0}^{n-1} \psi^{2} \mathcal{X}_{\{\psi<j\}} d \mu=\int \psi^{2} \sum_{j=0}^{n-1} \mathcal{X}_{\{\psi<j\}} d \mu$ $=\int \psi^{2} \sum_{j>\psi} \delta_{j}([0, n-1]) d \mu$, where $(*)$ holds because integrands have the same distribution, as previously discussed. Therefore, $\sum_{k=1}^{\infty} \frac{1}{n_{k}^{2}} \sum_{j=0}^{n_{k}-1} \operatorname{var}\left(\varphi_{j}\right) \leq \sum_{k=1}^{\infty} \frac{1}{n_{k}^{2}} \int \psi^{2} \sum_{j>\psi} \delta_{j}\left(\left[0, n_{k}-1\right]\right) d \mu \stackrel{(*)}{=}$ $\int \psi^{2} \sum_{k=1}^{\infty} \sum_{j>\psi} \frac{\delta_{j}\left(\left[0, n_{k}-1\right]\right)}{n_{k}^{2}} d \mu \stackrel{(* *)}{=} \int \psi^{2} \sum_{j>\psi} \sum_{k=1}^{\infty} \frac{\delta_{j}\left(\left[0, n_{k}-1\right]\right)}{n_{k}^{2}} d \mu$, where $(*)$ is due to dominated convergence theorem (2.1.26), considering that $\psi^{2} \sum_{k=1}^{\infty} \sum_{j>\psi} \frac{\delta_{j}\left(\left[0, n_{k}-1\right]\right)}{n_{k}^{2}} \leq \psi^{2} \sum_{k=1}^{\infty} \frac{n_{k}}{n_{k}^{2}} \leq \psi^{2} \sum_{k=1}^{\infty} \frac{1}{n_{k}} \leq$ $\psi^{2} \sum_{k=1}^{\infty} \frac{1}{e^{a k}}=\psi^{2} \frac{1}{e^{a}-1}$, which is integrable; and $(* *)$ is because the double summation converges absolutely, as we have just argued. Moreover, $\int \psi^{2} \sum_{j>\psi} \sum_{k=1}^{\infty} \frac{\delta_{j}\left(\left[0, n_{k}-1\right]\right)}{n_{k}^{2}} d \mu=\int \psi^{2} \sum_{j>\psi} \sum_{k=m_{j}}^{\infty} \frac{1}{n_{k}^{2}} d \mu$, where $m_{j}$ is the smallest index such that $n_{m_{j}}-1 \geq j$, because if $k<m_{j}, n_{k}-1<j$ and the term in the summation will have no contribution while, otherwise, the same term resumes to $\frac{1}{n_{k}^{2}}$.

Note that $\sum_{k \geq m_{j}} \frac{1}{n_{k}^{2}} \leq \sum_{k \geq m_{j}} e^{-2 a k}=e^{-2 a m_{j}} \sum_{k \geq 0} e^{-2 a k}=e^{-2 a m_{j}} \frac{1}{1-e^{-2 a}}<\infty(a>0)$. But $e^{a m_{j}} \geq j$, otherwise $e^{a m_{j}}<j<j+1 \leq n_{m_{j}}$ then $n_{m_{j}}$ would not be the smallest integer greater than $e^{a m_{j}}$. Hence $n_{m_{j}}>e^{a m_{j}} \geq j \Rightarrow \frac{1}{n_{m_{j}}{ }^{2}}<e^{-2 a m_{j}} \leq \frac{1}{j^{2}}$. Therefore $\sum_{j>\psi} \sum_{k \geq m_{j}} \frac{1}{n_{k}{ }^{2}} \leq \sum_{j>\psi} e^{-2 a m_{j}} \frac{1}{1-e^{-2 a}}=$ 
$\frac{1}{1-e^{-2 a}} \sum_{j>\psi} e^{-2 a m_{j}} \leq \frac{1}{1-e^{-2 a}} \sum_{j>\psi} \frac{1}{j^{2}} \underset{\psi \geq 3}{\leq} \frac{1}{1-e^{-2 a}} \int_{(\psi-2, \infty)} \frac{1}{s^{2}} d s=\left.\frac{1}{1-e^{-2 a}}\left[-s^{-1}\right]\right|_{\psi-2} ^{\infty}=\frac{1}{1-e^{-2 a}} \frac{1}{\psi-2}$, where $\int_{(\psi-2, \infty)} \frac{1}{s^{2}} d s$ and $\frac{1}{\psi-2}$ evaluated at $x$ mean, respectively, $\int_{(\psi(x)-2, \infty)} \frac{1}{s^{2}} d s$ and $\frac{1}{\psi(x)-2}$.

Summing up, we conclude that: $\epsilon^{2} \sum_{k=1}^{\infty} \mu\left(\left\{x \in X:\left|1 / n_{k} \sum_{j=0}^{n_{k}-1} \varphi_{j}(x)-\int 1 / n_{k} \sum_{j=0}^{n_{k}-1} \varphi_{j} d \mu\right|>\epsilon\right\}\right) \leq$ $\int \psi^{2} \sum_{j>\psi} \sum_{k=m_{j}}^{\infty} \frac{1}{n_{k}^{2}} d \mu \leq \int \psi^{2} \frac{1}{1-e^{-2 a}} \frac{1}{\psi-2} d \mu=\frac{1}{1-e^{-2 a}} \int \frac{\psi^{2}}{\psi-2} d \mu \stackrel{(*)}{\leq} \frac{1}{1-e^{-2 a}} \int \psi+6 d \mu=\frac{1}{1-e^{-2 a}}\left(6+\int \psi d \mu\right)<$ $\infty, \forall \epsilon>0$. Inequality $(*)$ holds because $\frac{\psi^{2}}{\psi-2} \leq \psi+6 \stackrel{\psi \geq 3}{\Leftrightarrow} \psi^{2} \leq(\psi-2)(\psi+6)=\psi^{2}+4 \psi-12 \Leftrightarrow 0 \leq 4 \psi-$ $12 \Leftrightarrow 3 \leq \psi$. As desired: $1 / n_{k} \sum_{j=0}^{n_{k}-1} \varphi_{j}-\int 1 / n_{k} \sum_{j=0}^{n_{k}-1} \varphi_{j} d \mu \rightarrow 0, \mu$-a.e.. Since $\int 1 / n \sum_{j=0}^{n-1} \varphi_{j} d \mu \rightarrow \int \psi d \mu$, it follows that $1 / n_{k} \sum_{j=0}^{n_{k}-1} \varphi_{j} \rightarrow \int \psi d \mu$. Denote the set of full measure where this convergence occurs by $X_{1}$.

As promised, we conclude showing why this $\mu$-a.e. convergence along a convenient subsequence implies standard $\mu$-a.e. convergence.

For each $x \in X_{1}$, we apply the lemma presented during the proof of 4.2.1: since $\varphi_{j}(x) \geq 0$ and $r=$ $\lim _{k} \frac{n_{k+1}}{n_{k}}=e^{a} \geq 1(a>0)$, it follows that $e^{-a} \int \psi d \mu \leq \liminf _{n} \frac{1}{n} \sum_{j=0}^{n-1} \varphi_{j}(x) \leq \lim \sup _{n} \frac{1}{n} \sum_{j=0}^{n-1} \varphi_{j}(x) \leq$ $e^{a} \int \psi d \mu$. Taking the limit as $a \rightarrow 0$, it follows that $\frac{1}{n} \sum_{j=0}^{n-1} \varphi_{j}(x) \rightarrow \int \psi d \mu, \forall x \in X_{1}$, as desired and concluding the main part of the argument.

The $\operatorname{limit}_{k} \lim _{k} \frac{n_{k+1}}{n_{k}}=e^{a}$ can be justified with the following arithmetic: $\lim _{k} \frac{n_{k+1}}{n_{k}}=\lim _{k} \frac{\left\lfloor e^{a(k+1)}\right\rfloor+1}{\left\lfloor e^{a k}\right\rfloor+1}=$ $\lim _{k} \frac{\left\lfloor e^{a(k+1)}\right\rfloor}{\left\lfloor e^{a k}\right\rfloor+1}=\lim _{k} \frac{1}{\frac{\left\lfloor e^{a k}\right\rfloor+1}{\left\lfloor e^{a(k+1)}\right\rfloor}}=\frac{1}{\lim _{k} \frac{\left\lfloor e^{a k}\right\rfloor+1}{\left\lfloor e^{a(k+1)}\right\rfloor}}=\frac{1}{\lim _{k} \frac{\left\lfloor e^{a k}\right\rfloor}{\left\lfloor e^{a(k+1)}\right\rfloor}}=\lim _{k} \frac{\left\lfloor e^{a(k+1)}\right\rfloor}{\left\lfloor e^{a k}\right\rfloor}$, but an analogous argument shows that $\lim _{k} \frac{\left\lfloor e^{a(k+1)}\right\rfloor}{\left\lfloor e^{a k}\right\rfloor}=\lim _{k} \frac{\left\lfloor e^{a(k+1)}\right\rfloor+\left\{e^{a(k+1)}\right\}}{\left\lfloor e^{a k}\right\rfloor\left\{e^{a k}\right\}}=\lim _{k} \frac{e^{a(k+1)}}{e^{a k}}=e^{a}$.

If we had, for $n \geq 0, \psi \circ f^{n} \geq 0$ but not $\psi \circ f^{n} \geq 3$, we could consider $3+\psi \circ f^{n}=(3+\psi) \circ f^{n} \geq 3$ in order to obtain: $1 / n \sum_{j=0}^{n-1}(3+\psi) \circ f^{j}(x) \rightarrow \int 3+\psi d \mu, \forall x \in X_{*}:=X_{3+\psi}$, where $X_{*}$ is a set of full measure. Then $1 / n \sum_{j=0}^{n-1} 3+\psi \circ f^{j}(x) \rightarrow \int 3+\psi d \mu \Leftrightarrow 3+1 / n \sum_{j=0}^{n-1} \psi \circ f^{j}(x) \rightarrow 3+\int \psi d \mu \Leftrightarrow$ $1 / n \sum_{j=0}^{n-1} \psi \circ f^{j}(x) \rightarrow \int \psi d \mu, \forall x \in X_{\star}$.

Finally, if not even $\psi \circ f^{n} \geq 0, n \geq 0$, is verified, consider $\psi=\psi^{+}-\psi^{-}$, where $\psi^{+}=\max \{\psi, 0\}$ and $\psi^{-}=\max \{-\psi, 0\}$. Then $1 / n \sum_{j=0}^{n-1} \psi \circ f^{j}(x)=1 / n \sum_{j=0}^{n-1}\left(\psi^{+}-\psi^{-}\right) \circ f^{j}(x)=1 / n \sum_{j=0}^{n-1} \psi^{+} \circ f^{j}(x)-\psi^{-} \circ$ $f^{j}(x)=1 / n \sum_{j=0}^{n-1} \psi^{+} \circ f^{j}(x)-1 / n \sum_{j=0}^{n-1} \psi^{-} \circ f^{j}(x) \rightarrow \int \psi^{+} d \mu-\int \psi^{-} d \mu=\int \psi d \mu, \forall x \in X_{\star}:=X_{\psi^{-}} \cap X_{\psi^{+}}$, where $X_{\star}$ is a set of full measure.

We should discuss what theorem 4.2.4 adds to results discussed in section 3.1. The basic comparison would be with theorem 3.1.7. However, before we compare them properly, we should try to extend theorem 3.1.7 to observables of infinite integral. as we do next. Please consider the same notation of theorem 3.1.7.

Theorem 4.2.5 (Bikhoff's ergodic theorem with ergodicity, v1, extended). Let $(X, \mathcal{A})$ be a measurable space, $f: X \rightarrow X$ be a measurable dynamical system and $\mu$ be a probability on $(X, \mathcal{A})$ such that $(f, \mu)$ is an ergodic system.

Then for all measurable $\psi: X \rightarrow \mathbb{R}$, with $\int \psi d \mu \in \overline{\mathbb{R}}( \pm \infty$ allowed $), \exists X_{\psi} \in \mathcal{A}, \mu\left(X_{\psi}\right)=1$, $1 / n \sum_{j=0}^{n-1} \psi \circ f^{j} \rightarrow \int \psi d \mu$, $\mu$-a.e. (in $X_{\psi}$ ).

Proof. We should only address the case $\int \psi d \mu= \pm \infty$. Suppose, without loss of generality, that $\psi \geq 0$ (so $\int \psi d \mu=\infty$ ).

Let $\psi_{m}=\min \{\psi, m\}, m \geq 1, X_{m}=X_{\psi_{m}}$, as obtained from theorem 3.1.7, and $X_{0}=\cap_{m \geq 1} X_{m}$. Clearly, $\mu\left(X_{0}\right)=1$ and, $\lim _{n} 1 / n \sum_{j=0}^{n-1} \psi_{m} \circ f^{j}(x)=\int \psi_{m} d \mu, \forall x \in X_{0}, m \geq 1$.

Choose any $x \in X_{0}$. Note that $\liminf _{n} 1 / n \sum_{j=0}^{n-1} \psi \circ f^{j}(x) \geq \liminf _{n} 1 / n \sum_{j=0}^{n-1} \psi_{m} \circ f^{j}(x), \forall m \geq$ 1. But $\liminf _{n} 1 / n \sum_{j=0}^{n-1} \psi_{m} \circ f^{j}(x)=\lim _{n} 1 / n \sum_{j=0}^{n-1} \psi_{m} \circ f^{j}(x)=\int \psi_{m} d \mu$. So considering $m \rightarrow \infty$, $\int \psi_{m} d \mu \rightarrow \int \psi d \mu=\infty$. Thus $\liminf _{n} 1 / n \sum_{j=0}^{n-1} \psi \circ f^{j}(x)=\infty$ and $\lim _{n} 1 / n \sum_{j=0}^{n-1} \psi \circ f^{j}(x)=\infty$, as desired.

Now, we focus on what theorem 4.2.4 adds to theorem 4.2.5. To support this discussion, we present theorem 4.2.4 as a corollary of theorem 4.2.5. 
Proof. As we know, the sequence $\varphi_{n}=\psi \circ f^{n}, n \geq 0$, is IID. Using the construction in example 4.1.4 and denoting the distribution of the $\varphi_{n}$ 's by $\nu=\varphi_{n_{*}} \mu$, we obtain a measure preserving function $\theta: X \rightarrow \mathbb{R}^{\mathbb{N}}\left(\mathbb{R}^{\mathbb{N}}\right.$ is considered with the product $\sigma$-algebra and the pushforward measure $\left.\theta_{\star} \mu=\nu^{\mathbb{N}}\right)$, an ergodic Bernoulli shift $\left(\sigma, \nu^{\mathbb{N}}\right)$ and a projection observable $\xi$, in such a way that they will serve as a model for $\left(\varphi_{n}\right)_{n \geq 0}$. In particular, it holds that $\varphi_{n}(x)=\xi \circ \sigma^{n}(\theta(x)), \forall x \in X$.

Since $\int \xi d \nu^{\mathbb{N}}=\int \xi d \theta_{\star} \mu=\int \xi \circ \theta d \mu=\int \varphi_{0} d \mu=\int \psi d \mu \in \overline{\mathbb{R}}$, we can apply theorem 4.2.5 to the ergodic system $\left(\sigma, \nu^{\mathbb{N}}\right)$ with the observable $\xi$. So we obtain a measurable set $Y_{\xi} \subset \mathbb{R}^{\mathbb{N}}, \nu^{\mathbb{N}}\left(Y_{\xi}\right)=1$, such that $1 / n \sum_{j=0}^{n-1} \xi \circ \sigma^{j}(x) \rightarrow \int \xi d \nu^{\mathbb{N}}=\int \psi d \mu, \forall x \in Y_{\xi}$.

The set $X_{*}=\theta^{-1}\left(Y_{\xi}\right)$ is measurable with $\mu\left(X_{*}\right)=1$. Clearly, $1 / n \sum_{j=0}^{n-1} \xi \circ \sigma^{j}(\theta(x)) \rightarrow \int \psi d \mu, \forall x \epsilon$ $X_{\star}$. However, $\xi \circ \sigma^{n}(\theta(x))=\varphi_{n}(x)=\psi \circ f^{n}(x)$, so $1 / n \sum_{j=0}^{n-1} \psi \circ f^{n}(x) \rightarrow \int \psi d \mu, \forall x \in X_{\star}$, as desired.

Therefore, theorem 4.2.4 can be viewed as a particular case of theorem 3.1.7. However, under practical terms, theorem 4.2.4, or at least the technique applied in the last proof, seems to say something new.

According to theorem 4.2.4, even if the system $(f, \mu)$ is not ergodic, we can conclude that the Birkhoff averages, observed trought a $\psi$ such that $(\psi,(f, \mu))$ is IID, converge to the average of the observable $\psi$. In example 4.1.5, one such case, where theorem 3.1.7 can not be applied directly, was presented.

What the last proof shows is that the former issue still boils down theorem 3.1.7 after using the trick of modeling the associated IID stochastic process with a Bernoulli shift, along the lines of example 4.1.4.

We conclude this chapter summarizing its major ideas. Our objective was to compare the law of large numbers with Birkhoff's ergodic theorem (assuming ergodicity).

In order to do so, we had to create a bridge between probability and ergodic theories. Their structures were combined into a single one, where the probability space considered is one equipped with an invariant measure with respect to a dynamical system acting of the space itself.

Attached in this space are random variables and observables, objects from probability and ergodic theories, respectively. More generally, we could consider stochastic processes and observables composed with iterates of the dynamics. A link between the last two was developed. Observables composed with iterates of the dynamics can be understood immediately as stochastic processes, and stochastic processes can be modeled by dynamical systems with an observable, in the sense of being equivalent in distribution to the observable composed with iterates of the dynamics.

This attitude allowed the concept of IID to be transported to ergodic theory. An observable is IID with respect to system whenever the associated stochastic process is IID. On the other hand, an IID stochastic process is modeled by a Bernoulli shift. Moreover, an IID system (one admitting a non trivial IID observable) has a Bernoulli factor. Similarly, ergodicity could have been transported in the reversed direction.

Although every IID system has as Bernoulli factor, not every IID system is equivalent to Bernoulli, as non-ergodic IID systems were presented. However, being non-ergodic does not imply low in a "chaos hierarchy". On the contrary, in some sense, IID systems should be considered at least Bernoulli in this hierarchy. In other words, asking for an IID system is still asking a lot from the system in consideration, although it might not necessarily be ergodic.

This bridge allowed the statement of the law of large numbers in dynamical systems terms, see theorems 4.2.3 and 4.2.4. Other theorems in probability theory can be adapted likewise. Those adaptations of the law of large numbers, theorems 4.2.3 and 4.2.4, were proved and compared with Birkhoff's ergodic theorem (assuming ergodicity). Note the process described up to this point is valuable on its own.

The conclusion of the comparison was that the (adapted) law of large number, theorem 4.2.3, could be obtained as a corollary of Birkhoff's ergodic theorem, theorem 4.2.5. Nonetheless, the (adapted) law of large numbers or the technique used to obtain it from Birkhhoff's ergodic theorem provides a new point of view, guaranteeing the conclusions of Birkhoff's ergodic theorem (with 
ergodicity) can hold for the non-ergodic case, at the cost of the observable being IID with respect to the system. 


\section{Chapter 5}

\section{Final Remarks}

\subsection{Conclusions}

The objective of the first part of this dissertation was to present a topological (or a topological dynamics) analogue to Birkhoff's ergodic theorem. This was accomplished to Birkhoff's ergodic theorem assuming ergodicity.

Using transitivity and recurrence rather than ergodicity, the mentioned analogue finds that a dramatic non-convergence of Birkhoff averages ${ }^{1}$ is the (topological) typical behavior, once these averages will accumulate in rather large set. This conclusion is opposed to the standard measuretheoretical typical convergence of Birkhoff averages due to Birkhoff's ergodic theorem (assuming ergodicity).

The above characterization is (invariant) measure-free and the extent of the former set is a topological property of the dynamical system. Whenever this set is the set of invariant measures, the system is called of maximal oscillation. Every point whose Birkhoff averages accumulate in the set of invariant measure is called a maximal oscillation point, i.e., a point of "worst" divergence for the Birkhoff average. Hence, in systems of maximal oscillation, (topological) typical points are of "worst" divergence. There are examples of both maximal and non maximal oscillation systems.

Under combined assumptions of Bikhoff's ergodic theorem (assuming ergodicity) and is analogue, considering a natural extra assumption, it also follows that the set of points whose Birkhoff averages diverge (called the irregular set) is topologically large but measure-theoretically negligible. In particular, the topological analogue describes the behavior of Birkhoff averages outside the domain of Birkhoff's ergodic theorem.

The objective of the second part of this dissertation was to compare the law of large numbers with Birkhoff's ergodic theorem (assuming ergodicity). In order to do so, a machinery allowing probability and ergodic theory to communicate was developed.

This machinery unified the objects of each theory and emphasized approaching dynamical systems equipped with an observable as stochastic processes and stochastic processes as modeled by dynamical systems equipped with an observable, in the sense of equivalence in distribution. In the same spirit, it defined an observable to be IID with respect to a system when the associated stochastic process was IID and showed that IID stochastic processes were modeled by Bernoulli shifts. In fact, IID systems (those admitting a non trivial IID observable) had a Bernoulli factor, even though they were not necessarily ergodic. Ultimately, this approach allowed the law of large numbers to be restated in an ergodic theory setting.

By doing so and performing the desired comparison, it comes out that the law of large numbers can be obtained as a corollary of Birkhoff's ergodic theorem. However, the law provides a new point of view, as it guarantees the conclusions of Birkhoff's ergodic theorem (assuming ergodicity) will hold even in the non-ergodic case, at the cost of requiring the observable is independent with respect to the system.

\footnotetext{
${ }^{1}$ The Birkhoff average being considered here is not $1 / n \sum_{j=0}^{n-1} \varphi \circ f^{j}(x)$, but $1 / n \sum_{j=0}^{n-1} \delta_{f^{j}}(x)$
} 


\section{$5.2 \quad$ Future studies}

Regarding the first part of the dissertation, a natural sequence of studies would be to look at when irregular sets and sets of maximal oscillation points are large, from the point of view of Baire category, topological entropy, topological pressure or Hausdorff dimension.

Additionally, one could look at the generalized topological analogue to Birkhoff's ergodic theorem assuming no ergodicity.

Regarding the second part of the dissertation, a natural sequence of studies would be to look at how other limit theorems from probability theory are translated to ergodic theory and whether good generalizations of them arise. Examples are the central limit theorem, the law of iterated logarithms and large deviation theory.

Additionally, a question which may be of interest to probabilists and readily approachable from this point is comparing the native definition of ergodicity from probability with that translated from ergodic theory. Similarly, translations of other ergodic theory concepts such as mixing, Kolmogorov systems, or even entropy and pressure, are possible. 


\section{Bibliography}

[BLV14] Luis Barreira, Jinjun Li, and Claudia Valls. Irregular sets are residual. Tohoku Mathematical Journal, 66(4):471-489, 2014. 67

[BS00] Luis Barreira and Jörg Schmeling. Sets of non-typical points have full topological entropy and full hausdorff dimension. Israel Journal of Mathematics, 116(1):29-70, 2000. 67

[Çın11] Erhan Çınlar. Probability and Stochastics, volume 261 of Graduate Texts in Mathematics. Springer, 1st edition, 2011. 3, 69, 75

[DGS76] Manfred Denker, Christian Grillenberger, and Karl Sigmund. Ergodic Theory on Compact Spaces, volume 527 of Lecture Notes in Mathematics. Springer, 1st edition, 1976. 64, 67

[EKL05] Chen Ercai, Tassilo Küpper, and Shu Lin. Topological entropy for divergence points. Ergodic Theory and Dynamical Systems, 25(4):1173-1208, 2005. 67

[Fol07] Gerald Folland. Real Analysis: Modern Techniques and Their Applications. Wiley, 2nd edition, 2007. 3

[Hoc] Michael Hochman. Notes on ergodic theory. Accessed online on 03.13.2018 at http: //math.huji.ac.il/ mhochman/courses/ergodic-theory-2012/notes.final.pdf. 60

[LW14] Jinjun Li and Min Wu. Generic property of irregular sets in systems satisfying the specification property. Discrete Continous Dynamical Systems, 34:635-645, 2014. 67

[LW16] Jinjun Li and Min Wu. Points with maximal birkhoff average oscillation. Czechoslovak Mathematical Journal, 66(1):223-241, 2016. 67

[Mor] Joel Moreira. Ergodic decomposition. Accessed online on 03.13 .2018 at https:// joelmoreira.wordpress.com/2013/09/20/ergodic-decomposition/. 60

[Oxt96] John Oxtoby. Measure and Category: A Survey of the Analogies between Topological and Measure Spaces, volume 2 of Graduate Texts in Mathematics. Springer, 2nd edition, 1996. 67

[Tao] Terence Tao. Ergodicity, lecture 9, 254a. Accessed online on 03.13.2018 at https://terrytao. wordpress.com/2008/02/04/254a-lecture-9-ergodicity. 60

[Tho10] Daniel Thompson. The irregular set for maps with the specification property has full topological pressure. Dynamical Systems, 25(1):25-51, 2010. 67

[VO16] Marcelo Viana and Krerley Oliveira. Foundations of Ergodic Theory, volume 151 of Cambridge Studies in Advanced Mathematics. Cambridge University Press, 1st edition, 2016. $3,13,21,24,45,60,61$

[Win] Reinhard Winkler. Upper semicontinuity of set valued functions and a topological counterpart of birkhoff's ergodic theorem (preprint). Prepint accessed online on 03.13.2018 at http://www.dmg.tuwien.ac.at/winkler/pub/semi.pdf. 64

[Win10] Reinhard Winkler. A little topological counterpart of birkhoff?s ergodic theorem. Uniform Distribution Theory, 5(1):157-162, 2010. 1, 61, 64 